\title{
AN ACTIVE THIN-FILM COCHLEAR ELECTRODE ARRAY WITH MONOLITHIC BACKING AND CURL
}

\author{
by \\ Angelique Candace Johnson \\ A dissertation submitted in partial fulfillment \\ Of the requirements for the degree of \\ Doctor of Philosophy \\ (Electrical Engineering) \\ in The University of Michigan \\ 2011
}

Doctoral Committee:

Professor Kensall D. Wise, Chair

Professor Bryan Pfingst

Professor Euisik Yoon

Associate Professor Katsuo Kurabayashi

Assistant Professor Mina Rais-Zadeh 
(C) Angelique Candace Johnson All Rights Reserved 2011 
To God and my Family 


\section{ACKNOWLEDGEMENTS}

I would like to acknowledge all those who have helped me along the way, but I feel I could write more than a few dissertations on that topic alone. So, I'll settle for acknowledging those who were most influential in helping me get to the doctorate program and through it.

I thank God for giving me the inspiration to attend graduate school, the strength to make it through it, and the sense to realize that this is just the beginning of the beginning of what he has for me to do.

I am very grateful to my committee members Dr. Kensall Wise, Dr. Bryan Pfingst, Dr. Euisik Yoon, Dr. Katsuo Kurabayashi and Dr. Mina Rais-Zadeh for their advisement and instruction. In particular, I would like to thank Dr. Wise. He supported and guided me through the process, but, at the same time, he gave me room to make my own mistakes and discoveries. He inspired me to go into MEMS, before I even joined his group and he kept me fascinated with the area long after. His enthusiasm for tackling the challenges in medicine and MEMS was infectious. He would always remind me that the purpose of my work was not to create something publishable, but to create something for the public. He emphasized the importance of letting the application of the technology drive the creation of it. Most importantly, Dr. Wise showed me that it is possible to be the best at what you do, be revered for what you do and still be a nice and a humble person. I could not have been given a better advisor and it is a blessing to have been able to work with him. In addition to my committee, I am also indebted to those who made it possible to demonstrate the usefulness and effectiveness of my work. I want to thank Deborah Colesa, Dr. John Middlebrooks, and Dr. Russell Snyder for all of their help and patience in conducting the in-vivo surgeries. For their assistance in the hybrid backing and bonding of the arrays I thank Alexander Hetherington, Dr. Radheshyam Tewari and Brendan Casey. 
I would like to thank my extended family, aunts, uncles, grandparents, nieces, nephews and in-laws. My Grandma and Poppa (James and Winona Goode) not only contributed to the makeup of what I am, but also helped make me who I have become. I pray that they are resting in peace and that I can continue to grow from the seed of their example. Thank you to all my siblings (by the names I have known them), Bicky, Junior, Lee, Morgan, Rozzy, Marty, Jeannine, Veronica, Maya and Damien for all that I have learned by them taking care of me and me taking care of them. They are the most influential people in my life, and had I not had their trail to follow I would not have found my way to and through this process. In particular I would like to thank Dr. Lee Johnson, Dr. Morgan Johnson and Dr. Martinique Johnson for showing me how it is done. Is there a Dr. in the house? Yes sir, plenty.

I would like to thank my parents, Charles and Veronica Johnson (Mommy and Daddy) for everything, for all that I have, and for all that I am. It all started with them and they keep it moving. Among many things, my father showed me what it meant to work hard no matter the difficulties and to remember God in all that you do. My mother taught me how to hustle for the things you need/want in life and to never give up, even beyond the last second. I could go on and on about all that my family has done for me and all that they mean to me. I love you all.

Last but not least, I would like to thank all of my colleagues and friends that made my time at Michigan productive and fun. Thank you to all the members, past and present, of the Society of Minority Engineers and Scientists, Graduate Component. You all truly were my family at the University, and I wouldn't have made it through without all the great food, laughs, fun and shoulders to learn on. In addition to those friends I made outside of work, I would like to thank all those friends I made while working through many hours in the lab and the cleanroom. A special thanks to the students of the Wise lab, Najafi lab, Gianchandani lab, and the NSF Wireless Integrated Microsystems Engineering Research Center. I hope to continue our friendship as I enter the next phase of my life. 


\section{TABLE OF CONTENTS}

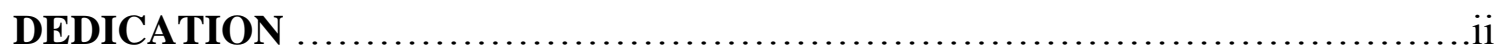

ACKNOWLEDGEMENTS _...........................................................

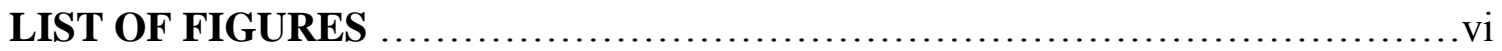

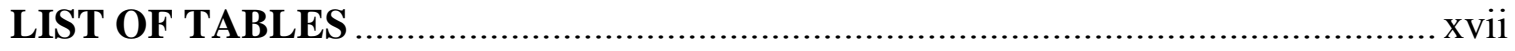

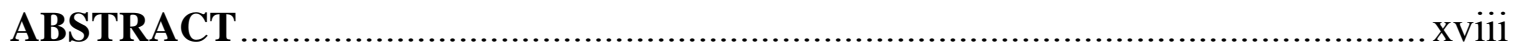

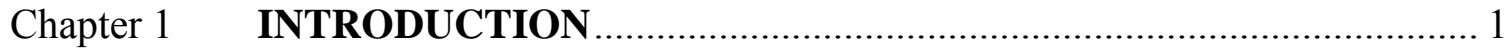

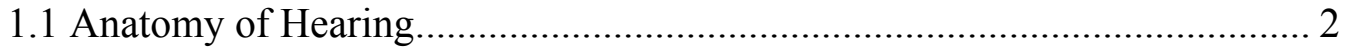

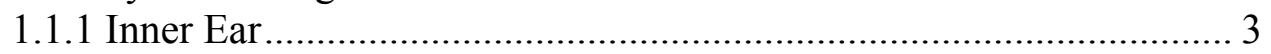

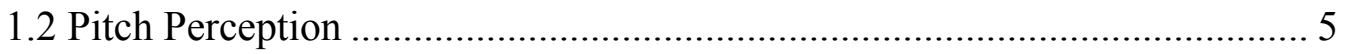

1.2.1 Phase Locking ………………………………………………..... 5

1.2.2 Tonotopic Mapping ...................................................................... 5

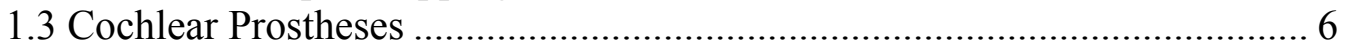

1.3.1 Speech Processing Strategies ....................................................... 7

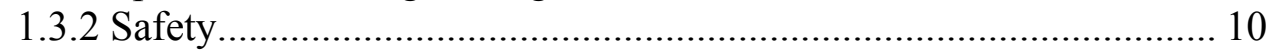

1.3.3 Frequency Selectivity ................................................................... 11

1.3.4 Commercial Prostheses .................................................................. 12

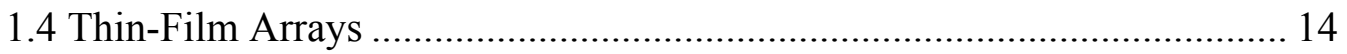

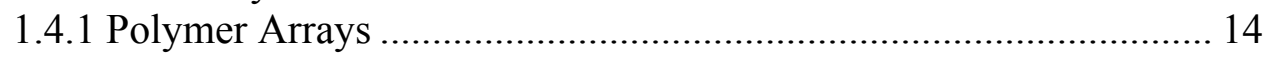

1.4.2 Silicon Arrays............................................................................. 16

1.4.3 Silicon-Polymer Hybrid Arrays ………………………………....... 19

1.4.4 Summary of Thin-Film Arrays.................................................... 21

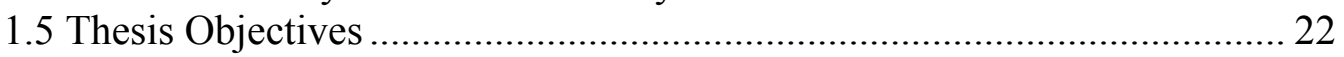

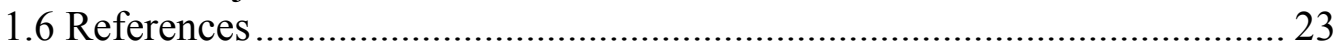

Chapter 2 DESIGN AND FABRICATION OF A PARYLENE COCHLEAR

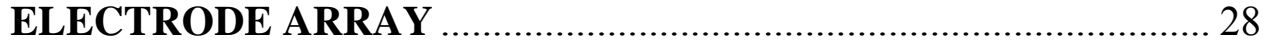

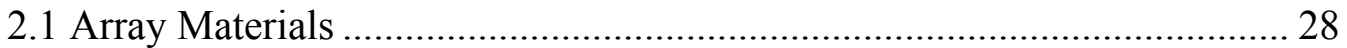

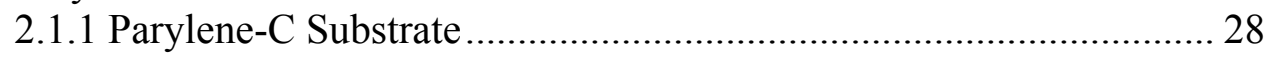

2.1.2 Iridium Oxide Electrode Sites ....................................................... 30

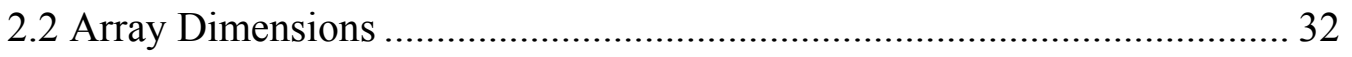

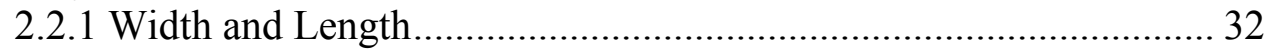

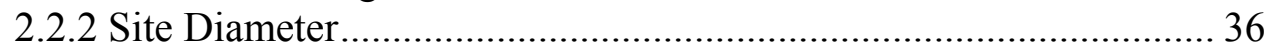

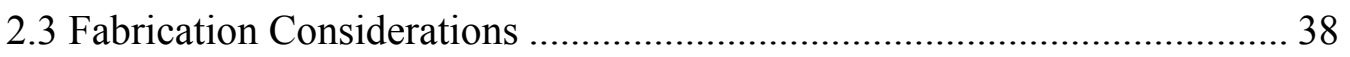

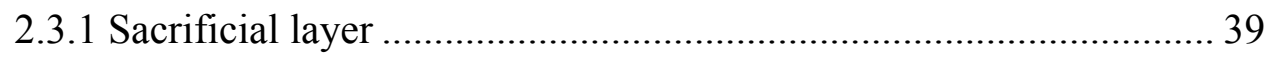

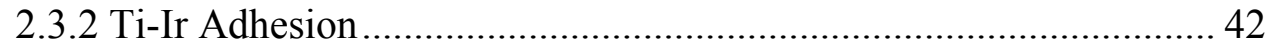


2.3.3 Interconnect Pitch......................................................................... 44

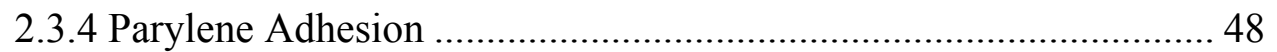

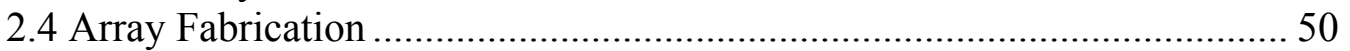

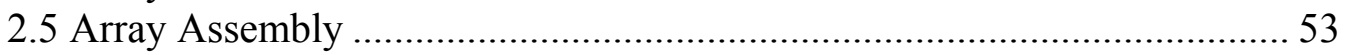

2.6 Electrical Performance ........................................................................... 54

2.7 Mechanical Performance......................................................................... 58

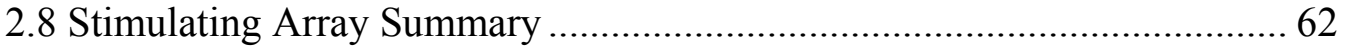

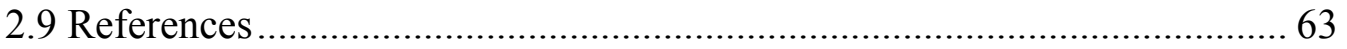

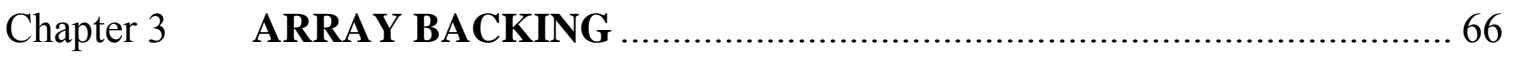

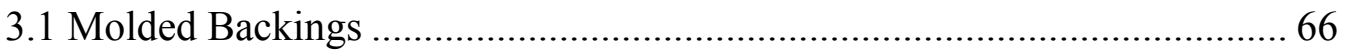

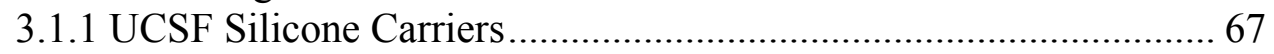

3.1.2 Cochlear Ltd. Silicone Carriers........................................................ 70

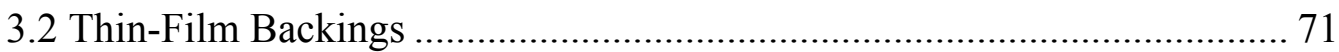

3.2.1 Hybrid ..................................................................................... 72

3.2.2 Monolithic Curl Frames ................................................................. 77

3.2.3 Monolithic Positioner-Stiffeners .................................................... 84

3.2.4 Backing Summary ................................................................. 104

3.2.5 References ............................................................................... 105

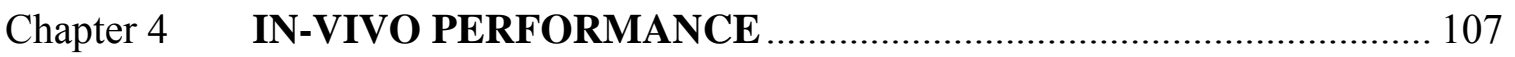

4.1 Surgical Procedure .................................................................................. 108

4.2 Stimulation and Recording Setup …………………............................ 110

4.3 Mechanical Performance........................................................................ 112

4.4 Performance using Electrical Stimulation............................................... 118

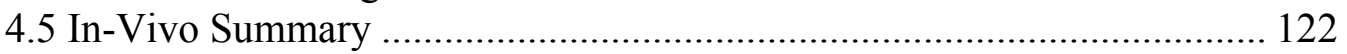

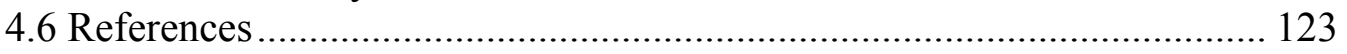

Chapter 5 ACTIVE ELECTRODE ARRAY …………………………........ 124

5.1 System Organization and Specifications................................................ 126

5.1.1 Stimulation Configurations ........................................................ 127

5.1.2 Site Manipulation and System Verification ................................... 130

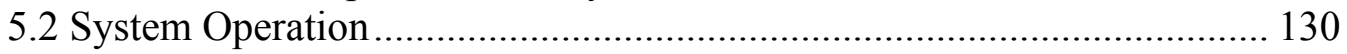

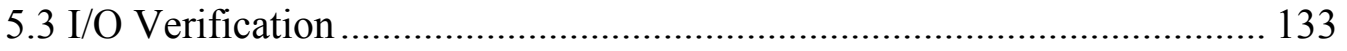

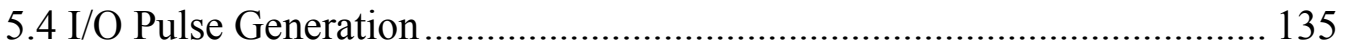

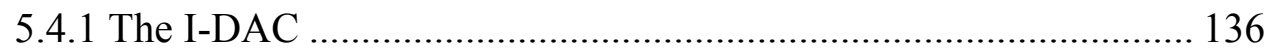

5.4.2 DAC Calibration ....................................................................... 141

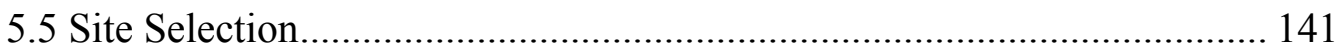

5.6 Site Manipulation and Characterization................................................... 142

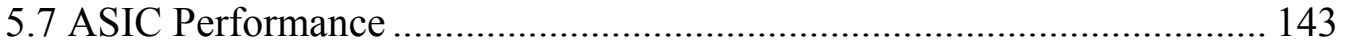

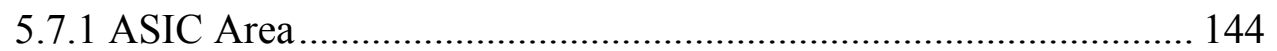

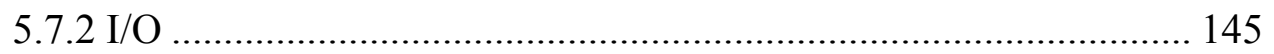

5.7.3 Stimulation Performance................................................................ 147

5.7.4 ASIC Performance Summary..................................................... 149

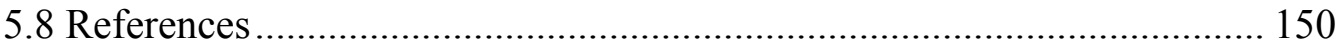

Chapter 6 SUMMARY, CONTRIBUTIONS, AND FUTURE WORK ….......... 152 


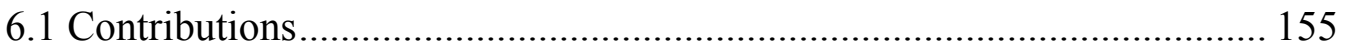

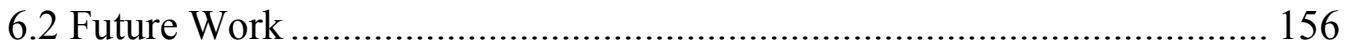




\section{LIST OF FIGURES}

Fig. 1.1: The outer, middle and inner ear regions of hearing anatomy [2] ...................... 2

Fig. 1.2: Cross section of the cochlea depicting the SV, cochlear duct and ST [2]........... 3

Fig. 1.3: Organ of Corti connected to the auditory nerve [4] . ........................................ 4

Fig. 1.4: Tonotopic mapping of the frequencies along basilar membrane. Frequencies get progressively lower going from base to apex [9] ........................................................... 6

Fig. 1.5: Cochlear prosthesis system with speech processor, transceiver coil and electrode

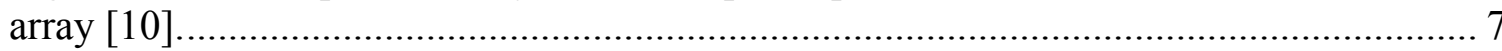

Fig. 1.6: (a) CA approach, with AGC, BPF's and Gain stages (b) CIS approach, with preemp BPFs, rectifiers, nonlinear map compressor and pulse train modulation [11]...... 8

Fig. 1.7: The Cochlear Ltd. Nucleus Contour array with speech processor, transceiver coil and electrode array [29] ..................................................................................... 12

Fig. 1.8: The Advanced Bionics HiFocus array with speech processor, transceiver coil and electrode array [30].

Fig. 1.9: The MED-EL Combi 40+ array with speech processor, transceiver coil and

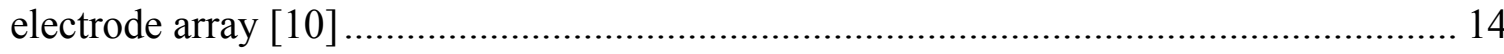

Fig. 1.10: The Advanced Cochlear Systems arrays comprised of stacked Au-patterned

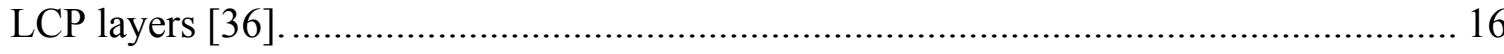

Fig. 1.11: The Michigan cochlear electrode array integrated with a polyimide cable to the

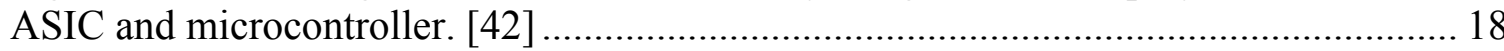

Fig. 1.12: A silicon - parylene - inorganic dielectric thin-film array with IrO sites for stimulation and piezoresistive strain gauges for position sensing [46] ............................ 19

Fig. 1.13: The IMEC active electrode array with silicon islands encased in silicone [ref].

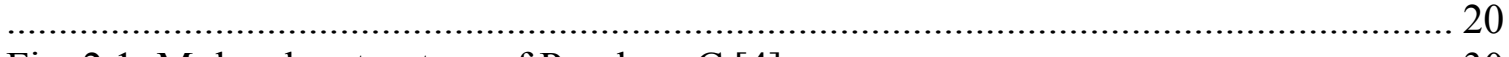

Fig. 2.1: Molecular structure of Parylene C [4] ............................................................ 30

Fig. 2.2: The Parylene- $\mathrm{C}$ deposition process [8] ............................................................ 30

Fig. 2.3: Layout dimensions of parylene substrate cat arrays........................................... 32 
Fig. 2.4: Layout dimensions of parylene substrate guinea pig arrays 33

Fig. 2.5: Span of the GP height and width as extracted from Voie-Spelman dimensional data. The GP array width is based on these constraints. . 34

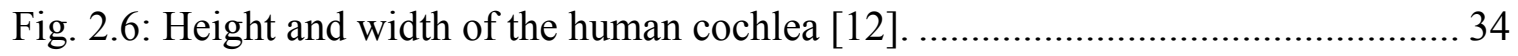

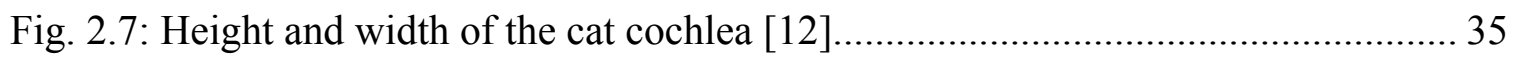

Fig. 2.8: Cross-sectional area of the cat cochlear relative to the human cochlea [12]...... 35

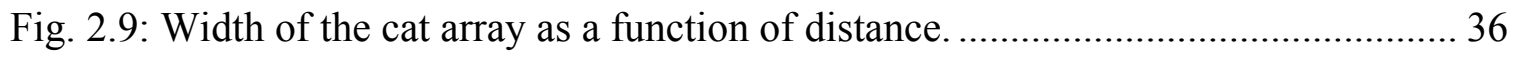

Fig. 2.10: Charge density as a function of electrode site diameter ............................... 36

Fig. 2.11: The Randles electrode model depicting the charge transfer resistance, double layer capacitance, and spreading resistance.......................................................... 37

Fig. 2.12: Simulated spreading resistance as a function of site diameter. ...................... 38

Fig. 2.13: Simulated back voltage in response to a $500 \mu \mathrm{A}$ stimulus pulse for $\mathrm{IrO}$ sites, versus diameter. Back-voltage includes contributions from spreading resistance and site-

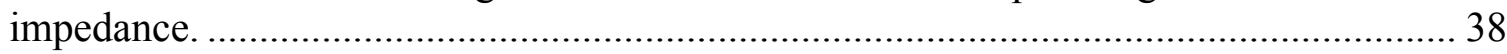

Fig. 2.14: Ti-Ir sites fractured and delaminated from parylene substrate, post release... 40

Fig. 2.15: Residual aluminum sacrificial layer under a cochlear array after more than

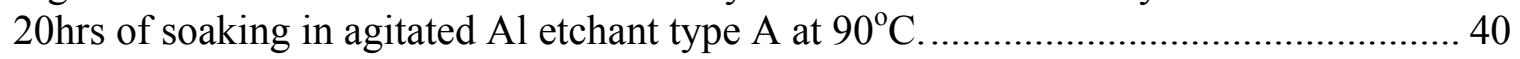

Fig. 2.16: Delamination of parylene from silicon surface due to lack of an adhesion

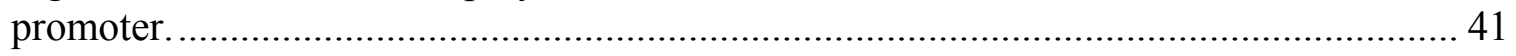

Fig. 2.17: Burst parylene substrate due to out-gassing of solvents............................. 42

Fig. 2.18: Buckling and fracturing of a Ti-Ir site under high compressive stresses greater

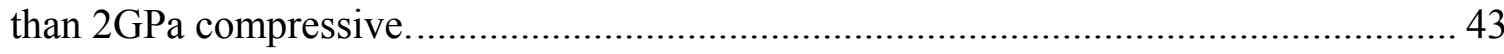

Fig. 2.19: Securely adhered Ti-Ir sites on parylene substrate arrays, post-release.......... 44

Fig. 2.20: Delaminated interconnect resulting from ultrasonic agitation of wafers in acetone.

Fig. 2.21: Single layer, standard lithography producing incomplete liftoff of $\mathrm{Cr}-\mathrm{Au}-\mathrm{Cr}$ on a parylene substrate...................................................................................... 45 Fig. 2.22: Standard lithography producing a $90^{\circ}$ side-wall angle, (a) $90^{\circ}$ side-wall angle in resist after exposure and development, (b) metal deposition coating the bottom of a trench 
more than the side-walls, (c) poor liftoff, with metal on side-walls clinging to trace lines.

Fig. 2.23: Standard lithography producing less than $90^{\circ}$ side-wall angles. (a) less than $90^{\circ}$ side-wall angle in resist after exposure and development, (b) metal deposition coating the bottom of a trench more than the side-walls, (c) poor liftoff, with metal on the side-walls and top surface of the resist clinging to the trace lines. 46

Fig. 2.24: Slowly developing the top resist with respect to the bottom produces recessed side-walls. (a) Difference in developing rate between top and bottom resist results in recessed side-walls, (b) No contact of metal on the remaining side-wall with traces at the bottom of the trench, (c) unwanted metal lifts off easily with resist, leaving clean trace lines behind.

Fig. 2.25: Surface "hardening" of resist to produce recessed side-walls. (a) Reduced solubility of the top surface producing recesses as the bulk etches faster, (b) No contact of metal on the remain side-wall with traces at the bottom of the trench, (c) unwanted metal lifts off easily with resist, leaving clean trace lines behind. 47

Fig. 2.26: TMAH liftoff of $\mathrm{Cr}-\mathrm{Au}-\mathrm{Cr}$ interconnect with $10 \mu \mathrm{m}$ pitch lines.

Fig. 2.27: Process flow for the fabrication of parylene electrode arrays. (a) a substrate layer of parylene is vapor deposited on a silicon carrier, (b) $\mathrm{Cr}-\mathrm{Au}-\mathrm{Cr}$ interconnect is evaporated and defined with a TMAH liftoff technique, (c) a dielectric layer of parylene is vapor deposited over the interconnect following an oxygen plasma ash, and contact openings are etched, (d) Ti-Ir electrode sites are sputter deposited at low base pressures and removed with TMAH liftoff, (e) following an oxygen plasma ash, a top coat of parylene is applied to the arrays and is oxygen plasma etched to remove the parylene from the sites and field areas.

Fig. 2.28: A 32-site parylene guinea pig array with close-up of two Ti-Ir sites. 52

Fig. 2.29: A 32-site parylene cat array. 52

Fig. 2.30: A parylene substrate cat array with a close-up of two Ti-Ir sites. 53

Fig. 2.31: An array backend and close-up of the ball bond area. 53

Fig. 2.32: Contact pads for ball bonding to the PCB. The exposed metal frame through the parylene allows threading of the ball bond to the PCB. 54

Fig. 2.33: Ball bonds going through contact frames on the array backend and connecting to the PCB. 
Fig. 2.34: Impedances of five sites across an array as a function of frequency (preactivation). For simplicity's sake, only five electrode impedances are shown. They are representative of impedances in neighboring sites as well. 55

Fig. 2.35: Impedances of five electrode sites across a parylene array as a function of frequency (pre-activation). For simplicity's sake, only five electrode impedances are shown. They are representative of impedances in neighboring sites as well.

Fig. 2.36: Ir site pre-activation. CV sweeps from $-0.6 \mathrm{~V}$ to $0.8 \mathrm{~V}$ at a rate of $100 \mathrm{mV} / \mathrm{s}$. The $\mathrm{x}$-axis is time (s) and the $\mathrm{y}$-axis is current (A).

Fig. 2.37: IrO electrode site, post-activation. Site was activated to a charge capacity of

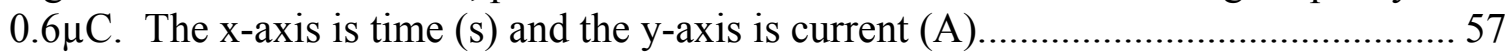

Fig. 2.38: Experimental set-up for cable bend-tests. ………....................................... 59

Fig. 2.39: Experimental set-up for cable twist-tests. ……………................................ 59

Fig. 2.40: Labview interface for setting flex-test parameters and recording impedance

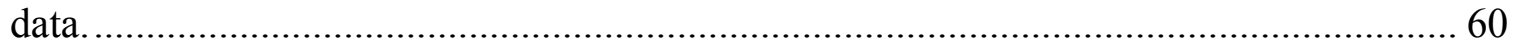

Fig. 2.41: Impedance of cable leads versus the number of bend-cycles. Impedance measurements were made during array bending. The impedance does not significantly drop with bending. Drop within the first 1000 cycles can be attributed to test set-up. ... 61

Fig. 2.42: Impedance of cable leads versus the number of twist-cycles. Impedance measurements were made during array twisting. The impedance does not significantly drop with twisting. Drop within the first 500 cycles can be attributed to test set-up. ..... 61

Fig. 2.43: The impedances between the cable lead lines and the saline solution over time. There is no significant difference in impedance pre- and post-twisting. Above graph is

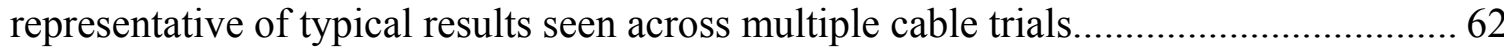

Fig. 3.1: The UCSF wire-bundle animal cochlear electrode array [3]............................ 67

Fig. 3.2: The UCSF stainless steel mold used to injection-mold silicone around the

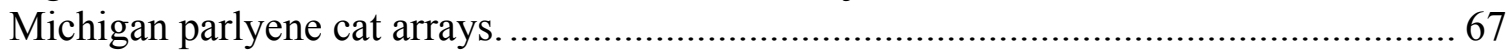

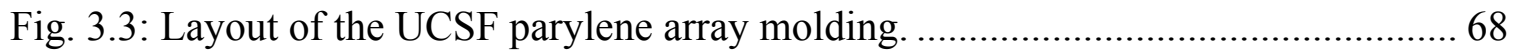

Fig. 3.4: Michigan parylene cat array in UCSF silicone carrier. ....................................... 69

Fig. 3.5: Michigan parylene cat array in Cochlear Ltd. silicone carrier. .......................... 71

Fig. 3.6: Michigan parylene cat array in Cochlear Ltd. silicone carrier. The parylene array has delaminated from carrier when straightened with a stylet-wire. 
Fig. 3.7: Assembled components of MTU PET backing for Michigan parylene arrays. . 73

Fig. 3.8: Pre-curved MTU backing on Michigan parylene cat array. 74

Fig. 3.9: Curvature of PET backing deformed as a result of multiple stylet-wire insertions 74

Fig. 3.10: Full insertion of a PET-backed cat array into the cat cochlea. 75

Fig. 3.11: Proposed hybrid Assembly of PLA stiffener, parylene array and PET insertion tool [6]. 76

Fig. 3.12: PLA integration into the parylene process ............................................. 76

Fig. 3.13: Hot embossed PLA over a parylene substrate [6] ..................................... 77

Fig. 3.14: Simulated beam curvature versus the ratio of top and bottom parylene layer

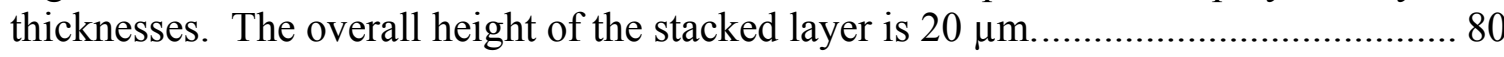

Fig. 3.15: Simulated radius of curvature versus the ratio of top and bottom parylene layer

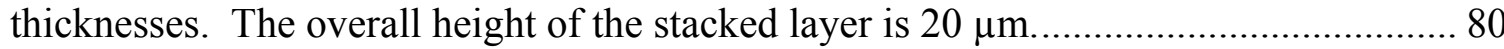

Fig. 3.16: Simulated beam curvature for increasing thickness of the top parylene layer over a $2 \mu \mathrm{m}$ thick bottom layer........................................................................ 81

Fig. 3.17: Simulated beam curvature for increasing thickness of the bottom parylene layer under a $2 \mu \mathrm{m}$ thick top layer. 81

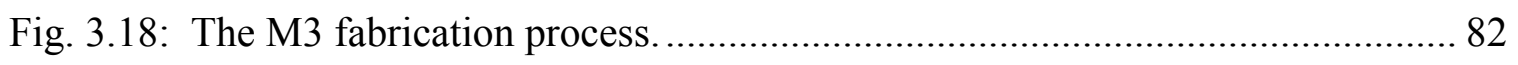

Fig. 3.19: Curled GP array with high stress Ti-Ir and Ti-Pt layers............................. 83

Fig. 3.20: Uniformly spaced rings on a slotted channel. ........................................ 85

Fig. 3.21: Non-uniformly spaced rings on a slotted channel. Gap length increases along the array. 85

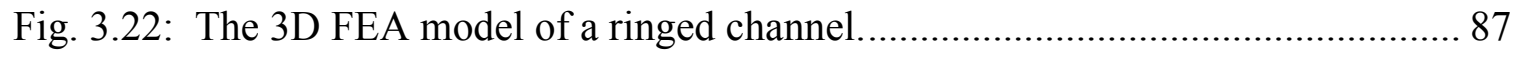

Fig. 3.23: Stiffness of the ringed-channel design versus the ring gap length for a constant

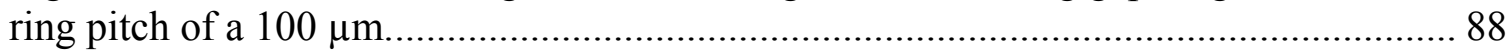

Fig. 3.24: Rigidity of the ringed-channel design versus the ring gap length for a constant

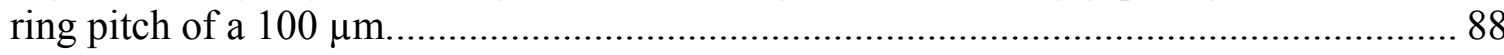

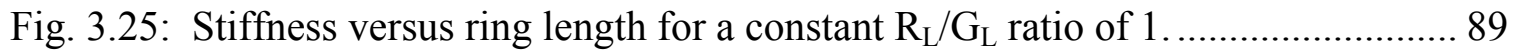


Fig. 3.26: Rigidity versus ring length for a constant $R_{L} / G_{L}$ ratio of 1 . 89

Fig. 3.27: Process flow for the fabrication of ringed-channel devices. 90

Fig. 3.28: Overlap of channel offset as a result of wet processing. 91

Fig. 3.29: A parylene channel with rings that have been over etched in the $\mathrm{O}_{2}$ plasma.. 92

Fig. 3.30: A ringed-channel array inserted into a guinea pig cochlea mold. The channel achieves the desired uniform bending profile. 93

Fig. 3.31: Curled guinea pig array with parylene rings integrated on back. 95

Fig. 3.32: Close-up of a curled guinea pig array with sites on one side and parylene rings on the other. 95

Fig. 3.33: A stylet-wire threaded through rings to straighten the pre-curved array for insertion into the cochlea. 96

Fig. 3.34: A $100 \mu \mathrm{m}$-thick copper wire pre-curling guinea pig array. 96

Fig. 3.35: A 3D FEA model of a discrete-ring channel.

Fig. 3.36: The stiffness of ringed-channel devices versus the ring gap length for a constant ring pitch of a $100 \mu \mathrm{m}$. 98

Fig. 3.37: The rigidity of a ringed-channel device versus the ring gap length for a constant ring pitch of a $100 \mu \mathrm{m}$. 98

Fig. 3.38: The stiffness versus the ratio of $R_{L} / G_{L}$ for a constant ring pitch of $100 \mu \mathrm{m} . .99$

Fig. 3.39: Rigidity versus the ratio of $\mathrm{R}_{\mathrm{L}} / \mathrm{G}_{\mathrm{L}}$ for a constant ring pitch of $100 \mu \mathrm{m}$. 99

Fig. 3.40: Stiffness versus the ring length for a constant $R_{L} / G_{L}$ ratio of 1 . 100

Fig. 3.41: Rigidity versus the ring length for a constant $R_{L} / G_{L}$ ratio of 1 100

Fig. 3.42: The fabriation process flow for discrete-ring devices. 101

Fig. 3.43: A pre-curved parylene array, backed with discrete rings 103

Fig. 3.44: A straight ringed array going around the first turn of the cochlea. Having no curvature the device does not hug the modiolus. 103 
Fig. 3.45: A pre-curved parylene array inserted through a cochlea mold. The curvature of the array allows it to hug the modiolus wall............................................................. 104

Fig. 4.1: Three electrode screws for EABR recording including ground, reference and active electrodes.

Fig. 4.2: Array positioned at entrance to cochlea through the bulla defect. A connector mounted on skull provides electrical access to the array sites. 110

Fig. 4.3: The PC array at the entrance to cat cochlea.

Fig. 4.4: A harvested cat cochlea after receiving a PC implant. The array can be seen jumping the scala media.

Fig. 4.5: A pre-curved and ring-backed (MC) array inserted $6.3 \mathrm{~mm}$ into GP cochlea with the aid of a graded-stiffness stylet wire. The array can be seen to cross over the scala media.

Fig. 4.6: A uniformly stiff, ring-backed (MC) array going through the middle ear space and into the cochleostomy.

Fig. 4.7: A uniformly stiff, ring-backed (US) guinea pig array entering cochlea. Array did not progress further than $4 \mathrm{~mm}$, as shown above. 116

Fig. 4.8: A graded-stiffness parylene (GS) array going around first turn of guinea pig cochlea. The array was housed entirely within the scala tympani.

Fig. 4.9: Cochlear America's animal array inserted into a guinea pig cochlea (a) and (b) a ring-backed, graded-stiffness (GS) parylene electrode array inserted similarly.

Fig. 4.10: Sample of neural activity recorded from EABRs in response to intracochlear stimulation of site number 21 using monophasic stimulus pulses with $200 \mu$ s phase durations.

Fig. 4.11: Sample of neural activity recorded from EABRs in response to intracochlear stimulation of site number 16 using stimulus pulses with $25 \mu$ s phase durations. 119

Fig. 4.12: Electrically evoked auditory nerve responses to stimulation with (a) Cochlear America's electrode array and (b) parylene electrode array. For $200 \mu \mathrm{A}$ pulses with monophasic, alternating polarity durations of $25 \mu \mathrm{s}$.

Fig. 4.13: Spike count versus the time following the onset of stimulation (in increments of $200 \mathrm{~ms}$ ). Neural firings in response to stimulation was picked up on recording sites 116 of a 16-site Neuronexus recording probe in the guinea pig IC in response to intracochlear stimulation of site number $21(50 \mu \mathrm{A})$. Activity is present on recording channels $2,5,8,9,12$ and 15 . 
Fig. 4.14: Spike count versus the time following the onset of stimulation (in increments of 200ms). Neural firings in response to stimulation was picked up on recording sites 116 of a 16-site Neuronexus recording probe in the guinea pig IC in response to intracochlear stimulation of site number $21(100 \mu \mathrm{A})$. Activity is present on recording sites $2,5,7,8,9,10,12,15$ and 16 122

Fig. 5.1: Previous generation ASIC [2] ………………...................................... 125

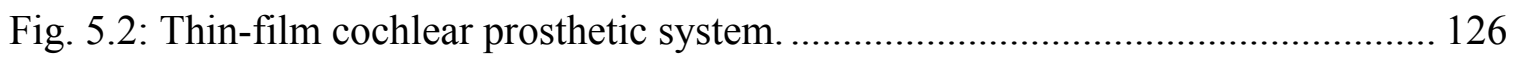

Fig. 5.3: An ASIC architecture where each channel is assigned to eight consecutive sites versus a distributed assignment where the channels are distributed in an alternating fashion among the sites.

Fig. 5.4: The charge between two sites, measured in medical Current Units (CU) is distributed equally, with $5 \mathrm{CU}$ per site (A), and in a ratio of $7 / 3$, with $7 \mathrm{CU}$ on one site and $3 \mathrm{CU}$ on the other (B). Two different frequencies can be perceived for (A) and (B) since two distinct cell groups are stimulated as depicted by the darker coloring [6].............. 128

Fig. 5.5: Two hardwired sites are used to create and additional three "virtual" sites by dividing the current across the sites in three different ratios. 129

Fig. 5.6: System-level view of the cochlear ASIC....................................................... 131

Fig. 5.7: 16-bit command word and bit designation. ................................................. 132

Fig. 5.8: Command sequence for a $500 \mu \mathrm{A}$ biphasic pulse. The pulse is generated with two stimulation commands followed by an extended-mode zero amplitude command. 133

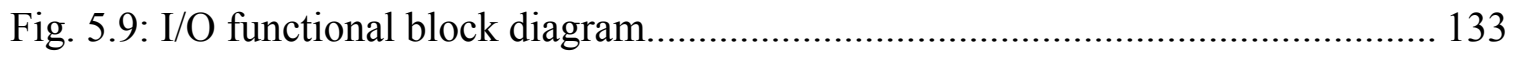

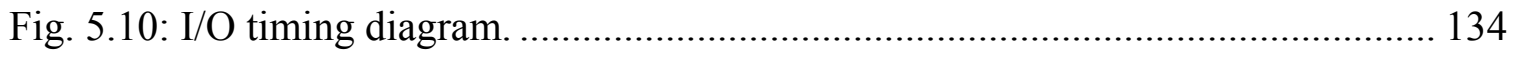

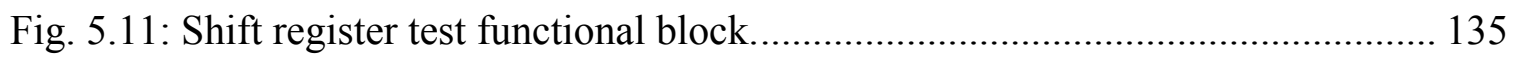

Fig. 5.12: Pathway of functional blocks used for channel selection............................... 136

Fig. 5.13: High-swing cascaded I-DAC..................................................................... 137

Fig. 5.14: Transistor sizing (in $\mu \mathrm{m}$ ) of voltage-biased current sources (in $\mu \mathrm{A}$ ) and DAC

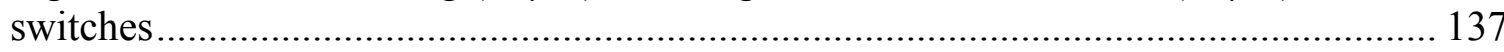

Fig. 5.15: Simulated Iout versus Vout driving $+500 \mu \mathrm{A}$ through a $3 \mathrm{k} \Omega$ resistor with a $\pm 2.5 \mathrm{~V}$ power supply .............................................................................................. 138 
Fig. 5.16: Simulated Iout versus Vout driving -500 $\mu \mathrm{A}$ through a $3 \mathrm{k} \Omega$ resistor with a $\pm 2.5 \mathrm{~V}$ power supply

Fig. 5.17: Progression of Iout with digital amplitude selection up to +500uA.............. 139

Fig. 5.18: Progression of Iout with digital amplitude selection down to $-500 \mathrm{uA}$........... 140

Fig. 5.19: Biphasic offset mismatch over-all output current selection (sourcing).......... 140

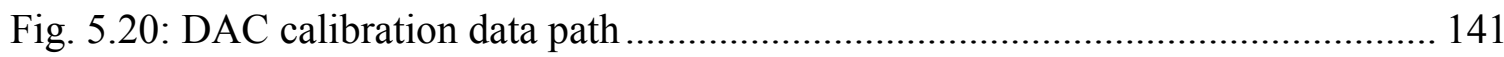

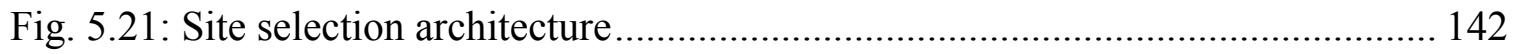

Fig. 5.22: Site impedance ground and activation......................................................... 143

Fig. 5.23: The Cochlear ASIC. Areas not outlined in white are consumed by interconnect. 144

Fig. 5.24: A 40kHz clock shifting in 1 on the DIN line through 16 clock cycles. DAV line alternates from high to low as the parity of the $\mathrm{I} / \mathrm{O}$ latch. The vertical scale is $5 \mathrm{~V} /$ div and the horizontal scale is $87 \mu \mathrm{s} / \mathrm{div}$ 146

Fig. 5.25: Output current versus supply voltage for a change in the supply voltage of $\pm 10 \%$. The amplitudes of VDD and VSS were equal but opposite. Fig. 5.26: Back voltage response to a $200 \mu \mathrm{s}$ and $300 \mu \mathrm{A}$ pulse. Backvoltage is roughly $0.3 \mathrm{~V}$ off a $0.5 \mathrm{k} \Omega \mathrm{IrO}$ site. The vertical scale is $100 \mathrm{~m} \mathrm{~V} / \mathrm{div}$ and the horizontal scale is $100 \mu \mathrm{s} / \mathrm{div}$.

Fig. 5.27: Back voltage response to a $25 \mu$ s and $128 \mu \mathrm{A}$ pulse. Backvoltage is roughly $0.13 \mathrm{~V}$ off a $0.5 \mathrm{k} \Omega \mathrm{IrO}$ site. The vertical scale is $100 \mathrm{mV} / \mathrm{div}$ and the horizontal scale is $20 \mu \mathrm{s} / \mathrm{div}$. 149 


\section{LIST OF TABLES}

Table 1.1: Comparison of Three Thin-Film Cochlear Electrode Array Technologies.... 22

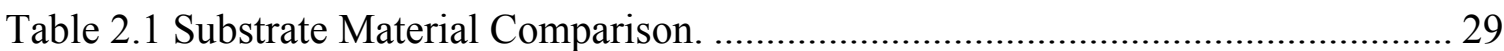

Table 5.1: Summary of ASIC Performance ................................................................... 150 


\section{ABSTRACT \\ AN ACTIVE THIN-FILM COCHLEAR ELECTRODE ARRAY WITH MONOLITHIC BACKING AND CURL \\ by}

\section{Angelique Candace Johnson}

Chair: Kensall D. Wise

Cochlear implants for the deaf are the most successful neural prostheses; however, pitch perception remains relatively poor. The size of the scala tympani into which the electrode array is inserted limits the number of electrodes to about twenty-four using conventional wire-bundle arrays. Thin-film arrays can offer significant advantages by increasing the number of sites, reducing damage to residual hearing with smaller and more flexible arrays, and allowing deeper insertion (greater pitch range).

This thesis creates an active cochlear array that builds on the success of a previous-generation Michigan array by increasing the reliability, usability and functionality. A robust and flexible 32-site prototype cochlear electrode array for a 128-site human array has been developed. Molded and thin-film backings for this array have been created for positioning the array inside the cochlea and close to the modiolar wall. The 
array has been integrated into the commercial molding process of Cochlear Ltd., as well as into a custom molding process created as part of this thesis. Batch-fabricated backings that include flexible parylene rings and self-curling parylene layers have also been created. These backings can achieve a minimum radius of curvature less than $0.5 \mathrm{~mm}$. Array stiffness can be graded from $0.2 \mathrm{k} \cdot \mathrm{N} / \mathrm{m}^{2}$ to $1.4 \mathrm{k} \cdot \mathrm{N} / \mathrm{m}^{2}$ with parylene rings to increase the rigidity seven-fold over that of a flat parylene array.

These parylene arrays have achieved full insertion into cat and guinea pig cochleae during in-vivo implants. The arrays have achieved the deepest insertions to date (more than $8 \mathrm{~mm}$ in some cases). Flexible guinea pig arrays achieved atraumatic implantations with no visible damage to the scala media.

A 32-site, 4-channel Application Specific Integrated Circuit (ASIC) was realized in $0.5 \mu \mathrm{m}$ technology to support a wide range of multisite multipolar stimulus configurations using a distributed Digital to Analog Converter (DAC) architecture. It fits within the space of the otic bulla having a size of $2.2 \mathrm{~mm}$ by $2.5 \mathrm{~mm}$, and operates from a $\pm 2.5 \mathrm{~V}$ supply at clock speeds up to $500 \mathrm{kHz}$. The maximum power consumption of the ASIC is $2.5 \mathrm{~mW}$ when outputting $500 \mu \mathrm{A}$ and it is compatible with back voltages exceeding $\pm 2 \mathrm{~V}$. 


\section{Chapter 1 \\ INTRODUCTION}

Cochlear prostheses are utilized to restore lost hearing to individuals suffering from sensorineural deafness. This type of hearing loss results from damage to the hair cells of the inner ear. Without these hair cells, the cochlea is unable to convert the mechanical vibrations of sound into electrical signals. Cochlear implants replace this lost transduction mechanism by electrically stimulating the neuronal processes of the auditory nerve.

Over 130,000 individuals have received cochlear prostheses to date [1] and they are the most widely adopted neural prosthetics, with a worldwide market projected to be $\$ 1.59$ billion by 2012 [1]. The two primary parts of the prosthesis are an external speech processor and an intracochlear electrode array. Although many advances have been made in the area of the speech processor, for the most part the electrode array design has gone through relatively few changes. In large part, this is because the size of the scala tympani into which the stimulating electrodes are inserted limits their number to about twenty using conventional wire-bundle manufacturing techniques. These arrays are also hand assembled and relatively expensive. Thin-film arrays can offer significant advantages by increasing the number of sites (increased pitch specificity), allowing deeper insertion (greater pitch range), and reducing cost. However, any thin-film array must be robust enough for safe insertion into the helical cochlea, stiff enough for deep insertion, and have a modiolus-hugging curl to position the sites close to the receptor cells and reduce insertion trauma.

Various thin-film cochlear arrays have been developed to address the issues of high site density and mechanical robustness. However, these devices have not fully addressed the problems associated with achieving successful array insertion. This research focuses on creating a thin-film cochlear electrode array that is not only robust 
with high site density, but is also integrated with curl and backing structures for successful placement inside the helical cochlea.

\subsection{Anatomy of Hearing}

The transduction of physical sound waves into electrical brain waves is a complex and not fully understood process. However, a basic understanding of how humans hear can be achieved by understanding the functions and interactions of the outer, middle and inner ear compartments. The outer, middle and inner ear components work together to convert acoustic waves into perceived sound (Fig. 1.1). Acoustic waves start out in the outer ear, a visible flap of cartilage, and get funneled through the ear canal. As the waves travel through the ear canal, they vibrate the tympanic membrane (ear drum). The middle ear contains a series of three small bones all connected together. As the ear drum moves, these bones amplify the force of the acoustic wave and transfer it to the fluid canals of the inner ear. As fluid moves within the inner ear, it sways tiny hairs whose motion sends neuronal impulses to the brain. It is the brain's translation of these impulses that results in perceived sound.

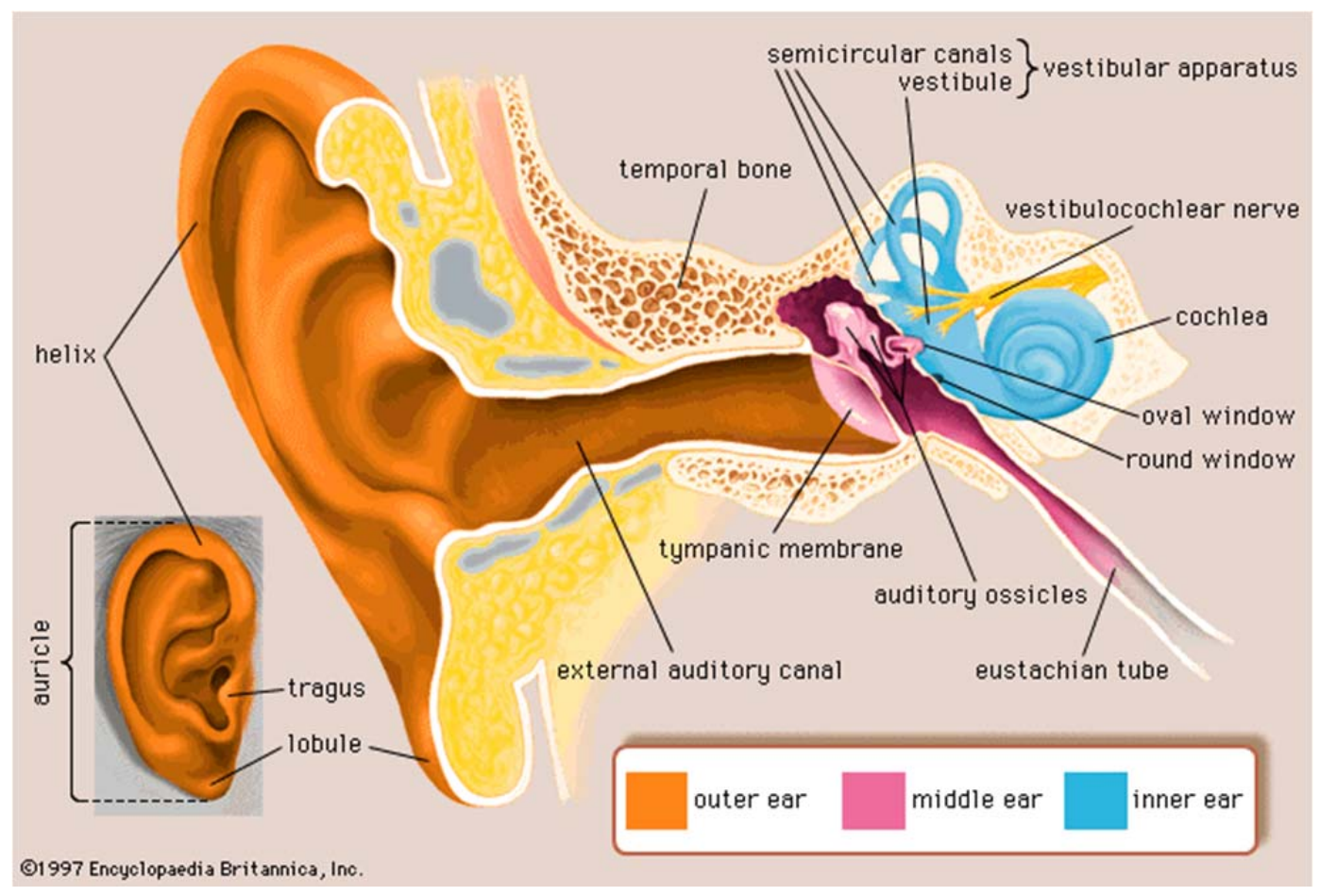

Fig. 1.1: The outer, middle and inner ear regions of hearing anatomy [2]. 


\subsubsection{Inner Ear}

The inner ear is where the transduction of mechanical sound vibrations to electrical neural signals occurs. There are two sides to the inner ear; the vestibular and the cochlear. The vestibular side primarily controls the body's sense of balance through fluid movement in semicircular canals and acceleration through the movement of otiliths (rocks) in small saccules. The cochlear side of the inner ear consists of the cochlea and controls hearing. It is this side that is the concern of cochlear prostheses, and it is what will be discussed here.

The cochlea is the small snail-shaped bone of the inner ear, positioned deep within the temporal bone. It is responsible for the mechanical to electrical (mechanoelectrical) transduction of acoustic waves into neural signals. The bone consists of a helical channel approximately $35 \mathrm{~mm}$ in length [3], which makes 2.5 turns around a porous cavity called the modiolus. This channel is separated into the three ducts: the scala vestibule (SV), the scala tympani (ST) and the scala media (SM), which is also known as the cochlear duct. Reissner's membrane separates the SV from the SM, and the basilar membrane separates the ST from the SM. While Reissner's membrane is primarily for fluid and electrolyte transport between the cochlear duct and SV, the basilar membrane is more directly involved in the mechanisms of hearing.

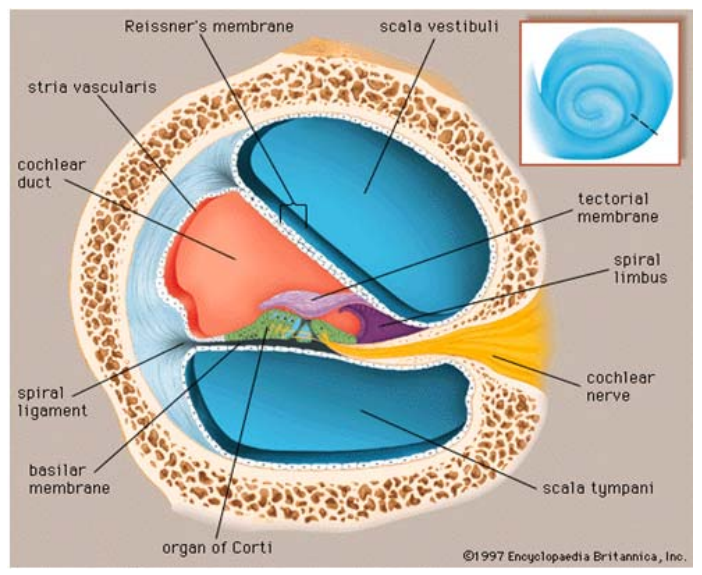

Fig. 1.2: Cross section of the cochlea depicting the SV, cochlear duct and ST [2]. 
The basilar membrane contains the organ of corti (Fig. 1.2) which houses the hair cells that perform the mechanoelectrical transduction of sound vibrations into neural impulses. This transduction occurs when the oval window fluctuates in response to middle ear amplification. This window is in contact with the fluid of the SV (perilymph) and its fluctuations cause waves in the perilymph which get transmitted to the ST by way of a small opening at the apex of the cochlea. As the wave moves through the scala tympani, it ripples the basilar membrane. The organ of corti, being attached to the membrane, moves causing the hair cells to sway. As the hair cells sway, their movement transmits sound information in the form of neural impulses to the brain. A closer look at the hair cell structure (Fig. 1.3) can help to explain how this mechanical movement translates to electrical impulses.

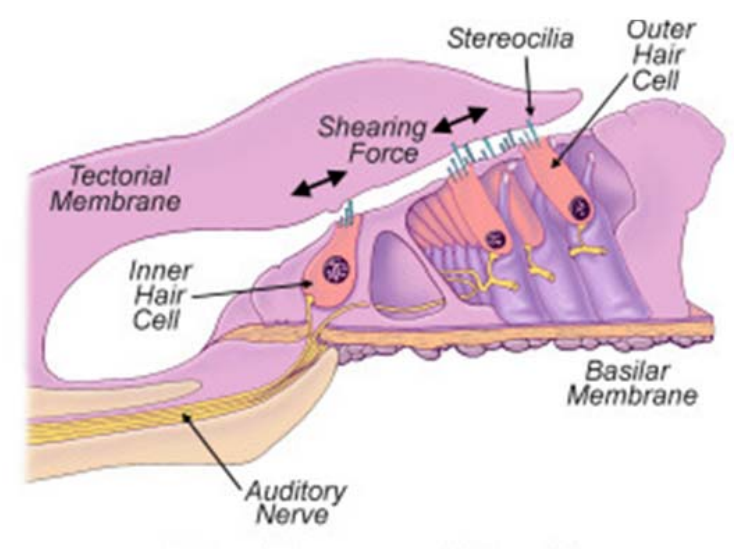

Fig. 1.3: Organ of Corti connected to the auditory nerve [4].

There are four rows of hair cells in the organ of corti, one inner and three outer. Although they account for only about $23 \%$ of the hair cells [5], most of the afferent nerve fibers $(85-95 \%)$ that transmit neural pulses to the brain are connected to the inner hair cells [6]. As such, these hair cell types are the primary mechanoelectrical transducers of the cochlea. The afferent nerve fibers are the peripheral processes of the spiral ganglion cells. Spiral groupings of these fibers extend off from the auditory nerve in the modiolus and wind through the osseous spiral lamina (OSL). Through the pores of the OSL they further branch off into the organ of corti and make synaptic contact to the hair cells. Cochlear hair cells contain tiny hair like protrusions (stereocilia), which extend into the SM and cylindrical cell bodies that form synaptic connections to the neuronal processes of spiral ganglion nerve cells. When the stereocilia sway in response to basilar 
membrane movement, their motion causes transconductance channels at their tips to open, and an influx of ions depolarizes the cell. In response to depolarization, the hair cells release neurotransmitters to the nerve fibers that excite action potentials in the ganglion cells. The axons of the ganglion cells regroup inside the modiolus at the auditory nerve and transmit the action potentials first to the cochlear nucleus (midbrain) and then onto the auditory cortex of the brain.

\subsection{Pitch Perception}

Frequency perception within the cochlea is not fully understood; however, it is thought to be dependent mostly on the rate of stimulation and place of stimulation.

\subsubsection{Phase Locking}

The link between the rate of stimulation and frequency perception is called phase locking. In response to an acoustic wave, a mechanical wave goes down the basilar membrane. The peaks of actuation in the membrane correspond to the peaks of pressure in the acoustic wave. Since the firing of neurons is directly related to the peak movement of the basilar membrane, when the nerves fire they fire in phase with the sound wave. This phase locking feature transmits frequency information through the auditory nerve [5]. However, as the frequency of the acoustic wave increases, individual nerve cells are unable to keep up because they have a refractory period of about $2 \mathrm{~ms}$, which limits their frequency of firing to about $500 \mathrm{~Hz}$. While this frequency restriction is placed on individual nerve cell fibers, a broader range of frequencies $(1.5 \mathrm{kHz}-3 \mathrm{kHz})$ can be translated by the temporal summation of action potentials from a collection of cells [7].

\subsubsection{Tonotopic Mapping}

The link between frequency and place of actuation along the basilar membrane was first discovered by Von Bekesy and is called tonotopic mapping. The basilar membrane thickness and width vary from base to apex. At the base of the cochlea, the membrane is thick and narrow. Going further into the cochlea towards the apex, the membrane becomes thinner and wider. This progression in the physical properties of the 
membrane makes it stiffer at the base than the apex. As such, when fluid waves move through the cochlea, low frequency waves achieve maximum actuation in the apical regions, whereas high frequency waves achieve maximum actuation at the base (Fig. 1.4). Thus, the locations at which ganglion cell processes are connected to mechanically actuated hair cells determine the frequencies those cells respond to best [8].

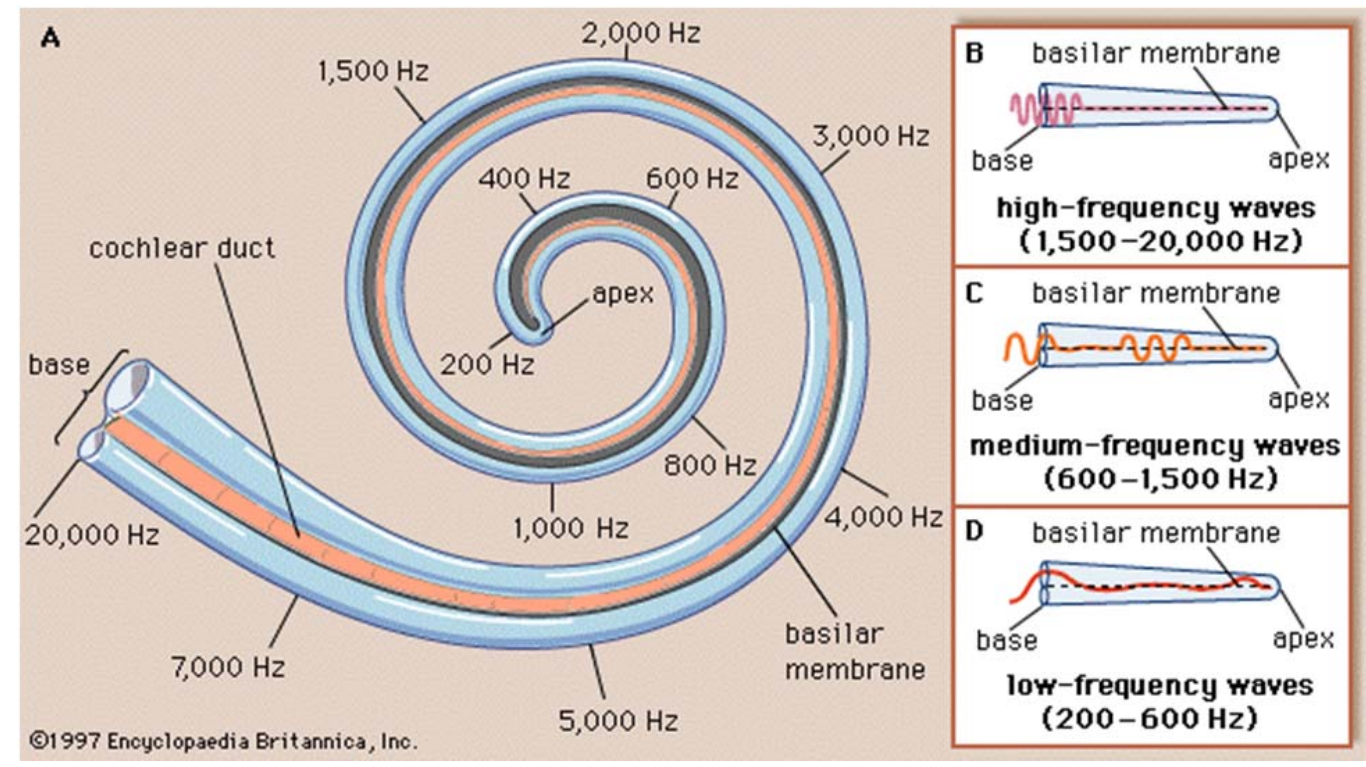

Fig. 1.4: Tonotopic mapping of the frequencies along basilar membrane. Frequencies get progressively lower going from base to apex [9].

\subsection{Cochlear Prostheses}

Cochlear prostheses use electrode arrays to replace the function of damaged or deadened hair cells. Through these electrodes, the frequency and volume content of sound are translated to the nerve cells. Frequency is translated through the tonotopic mapping of the cochlea where each electrode location corresponds to a particular frequency set. Volume translated by increasing or decreasing the amplitude of current stimulation. There are four components that allow a cochlear prosthesis to make this translation (Fig. 1.5). These components are (1) an external microphone to pick up the external acoustic information, (2) an external speech (signal) processor which decodes the microphone input into a set of relevant frequencies and corresponding intensities, (3) an external transmitter coil that sends frequency and stimulation commands to an implanted receiver-stimulator under the skin, which passes current pulses to the electrode 
array, and (4) an electrode array implanted into the cochlea that stimulates spiral ganglion cells, bypassing the non-functional hair cells. Components (2) and (4) are the main functional blocks of the prosthesis, and their operation will be discussed in the following sections.

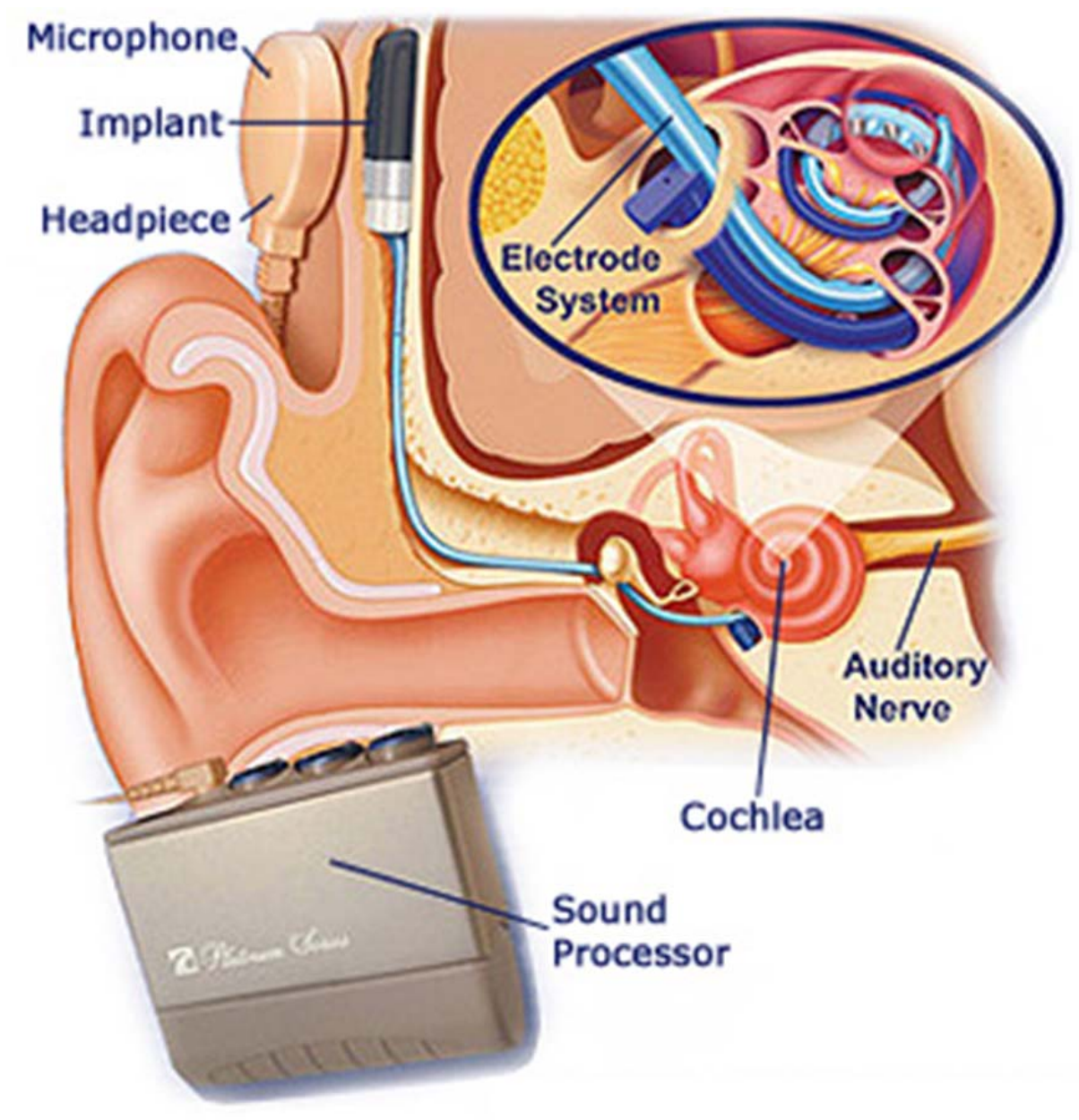

Fig. 1.5: Cochlear prosthesis system with speech processor, transceiver coil and electrode array [10].

\subsubsection{Speech Processing Strategies}

When it comes to representing auditory information, the focus of cochlear prosthetics is in the translation of speech content. This translation is done through a variety of algorithms, which use the auditory signals collected by the microphone to select which electrodes in the array get stimulated. Compressed-Analogue (CA) and 
Continuous Interleaved Sampling (CIS) are the primary speech algorithms used in speech processors (Fig. 1.6).
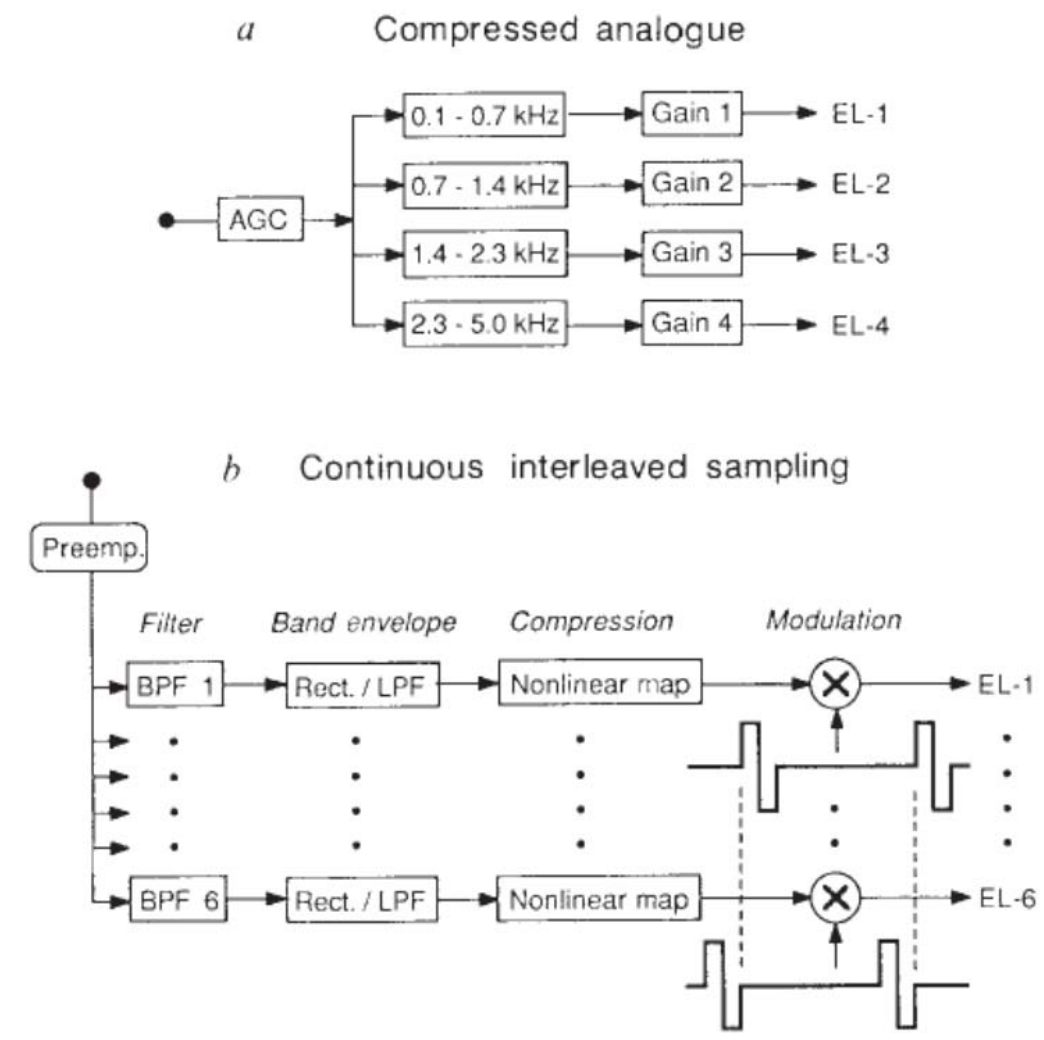

Fig. 1.6: (a) CA approach, with AGC, BPF's and Gain stages (b) CIS approach, with preemp BPFs, rectifiers, nonlinear map compressor and pulse train modulation [11].

The CA approach (Fig. 1.6a) was first presented by the University of Utah [11, 12]. This approach begins with an automatic gain control stage (AGC). The AGC is used to compress the broader dynamic range of the audio input to a much narrower electrical stimulation dynamic range. Following this AGC stage, the frequency content of the signal is broken into four separate frequency channels. The outputs of these bandpass filters are amplified and simultaneously transmitted through the electrodes. The advantage of the CA approach is that it is able to preserve the temporal information of the original waveform. At low frequencies this temporal information aids in pitch perception through phase locking. However, above $300 \mathrm{~Hz}$ the perceived pitch does not follow the temporal cues of analog stimulation [13]. Additionally, the simultaneous stimulation of the CA approach increases channel interactions which reduce frequency resolution [14]. 
To reduce channel interactions the CIS approach was developed by the Research Triangle Institute [15].

The CIS approach stimulates non-simultaneously using biphasic pulses that have been modulated by the envelope of the audio signal (Fig. 1.6b). The approach begins with the pre-emphasizing of the audio signal. This pre-emp stage emphasizes the high frequency (consonants) content in speech above $1.2 \mathrm{kHz}$ by selectively attenuating low frequency content (vowels) below $1.2 \mathrm{kHz}$ [13]. Without this step, the low frequency content would mask out the high. Following the pre-emp stage, the signal goes through a set of bandpass filters, which distribute the frequencies in tonotopic order among the electrode sites. The envelopes of the filtered waveforms are then extracted using a rectifier and low-pass filter. The low-pass filters are typically designed to have cut off frequencies of $200-400 \mathrm{~Hz}$ [16]. As in the CA approach, compression of the audio signals is necessary to convert the wide dynamic range of auditory stimulation to the narrow dynamic range of electrical stimulation. Compression of the envelopes is achieved using a nonlinear transformation (log or power law). Lastly, the compressed envelopes are used to modulate the amplitudes of biphasic pulse trains.

Although CA strategies are still used, almost all of the present cochlear prostheses use CIS or some variation of it. The most commonly used variations rely on an $n$-of- $m$ strategy.

The $n$-of- $m$ strategy is almost identical to CIS, but has an added channel selection scheme. Whereas with the CIS approach, all BPF channels were passed on to electrodes, in the $n$-of- $m$ approach only a subset of channels ( $n$ ) containing the $n$ highest envelope peaks are stimulated from $m$ total channels. The range of $n$ varies from processor to processor. The value of $n$ is dynamic and can change depending on how many channels have signals above noise level and also on the bandwidth of the audio signal. For example, in broadband audio signals, $\mathrm{n}$ is set to its maximum value to capture the wide range of frequencies. The dynamic control of $\mathrm{m}$ allows the processor to optimize speech perception by eliminating low intensity (i.e., content) frequencies and increasing stimulation rates which are dependent on the number of active channels. 


\subsubsection{Safety}

Safety protocols relate to the aspects of array insertion and tissue stimulation. Cochlear electrode arrays are inserted into the scala tympani of the cochlea through a drilled opening made close to the round window. As the array is advanced through the opening and down the scala tympani, care must be taken to not damage its physiology. In particular, trauma, which leads to ganglion cell destruction, can reduce residual auditory hearing and the effectiveness of electrically evoked hearing. Destruction of the ganglion cells can come as a result of basilar membrane tearing, fracturing of the osseous spiral lamina or loss of hair cells [17]. In most cases these events lead indirectly to ganglion cell death. While surgical techniques can limit the amount of insertion trauma, the mechanical structure of the array itself is the limiting factor.

The main mechanical attribute of the arrays in relation to safety is their stiffness. The stiffer an array is the higher the contact forces on the ST wall during insertion [18]. If the contact forces are too high they can cause trauma to the cochlea. The distribution of stiffness down the array also plays a role in contact forces. It has been shown that arrays with graded stiffness apply less contact force at their tips [19]. However, if the array is too flexible it can curl back on itself during insertion [20].

In addition to mechanical factors, electrical stimulation plays a role in safety as well. During the electrical stimulation of cochlear nerve cells, charge is transferred from the electrodes through the tympanic fluid to the nerve cell processes. This charge transfer occurs with reversible (oxidation-reduction) Faradic chemical reactions. To ensure that no toxic products are built up in the cochlea, biphasic current pulses are used to ensure full oxidation-reduction cycles occur. In addition to charge neutralization, charge densities per unit area play a large role in stimulation safety. If the charge densities are too high they produce the irreversible Faradic reactions, which lead to hydrogen and oxygen evolution (electrolysis). Toxic products and electrolysis can lead to undesirable $\mathrm{pH}$ changes, metal dissolution or the creation of metal protein complexes [17]. 


\subsubsection{Frequency Selectivity}

Electrically evoked hearing relies mostly on the tonotopic organization of the cochlea to translate frequency content from speech. The goal of each channel of stimulation is to stimulate narrow clusters of adjacent neurons which all represent the same frequency. The number of electrodes used in each channel and their orientation to each other can significantly affect the spread of current. The widely investigated configurations of electrodes in each channel are monopolar, bipolar and tripolar. In the monopolar configuration only one electrode is allocated to each channel. Biphasic pulses are stimulated through this electrode and returned to a distant ground placed outside the cochlea. Bipolar and tripolar configurations stimulate with two and three adjacent electrodes per channel, respectively. In these configurations, a potential difference is set up between adjacent electrodes, such that the electrical fields are much more confined over the sites than in the monopolar configuration.

Monopolar was the original mode of stimulation among early implant technologies. Monopolar stimulation produces broad current spread where multiple frequency sets of nerve cells are activated at the same time, with low frequency selectivity [21]. With the introduction of multielectrodes, investigations began on the effectiveness of multipolar stimulation from one channel [22]. Both bipolar and tripolar configurations have been shown to reduce current spreading, with tripolar achieving the highest degree of selectivity [23-25] at the cost of higher power consumption. Additionally, with bipolar and tripolar configurations, reducing the spacing between adjacent electrodes can further reduce the spread of current [23, 25]. Multipolar site configurations are referred to as $\mathrm{BP}+\mathrm{N}$ and $\mathrm{TP}+\mathrm{N}$, where $\mathrm{N}$ stands for how many sites separate adjacent pairs or triplets. A BP +0 configuration is one in which two directly adjecent sites with zero sites in between them are stimulated. The same goes for a $\mathrm{TP}+0$ configuration. The the smaller $\mathrm{N}$ is the more selective stimulation becomes. Bipolar configurations have been exploited to create virtual channels out of hardwired electrode sites $[26,27]$. Using these virtual channels it is possible to produce 127 perceived pitches using only 22 electrode sites [28]. 


\subsubsection{Commercial Prostheses}

Cochlear Ltd., Advanced Bionics and MED-EL are the three major manufacturers of cochlear implants today. Each manufacturer has a unique electrode array technology and speech processor. Below is an overview of the standard technologies for each company.

Cochlear Ltd. presently makes the Nucleus 24 and the Nucleus 24 Contour stimulating arrays (Fig. 1.7). Both arrays use bundles of 24 Teflon-coated platinum/iridium wires with welded bands of metal for electrode sites. The band electrodes are $0.3 \mathrm{~mm}$ in width with center-to-center spacings of $0.45 \mathrm{~mm}$. The diameters of the Nucleus 24 and Nucleus 24 Contour arrays taper from $0.6 \mathrm{~mm}$ to $0.4 \mathrm{~mm}$ and from $0.8 \mathrm{~mm}$ to $0.6 \mathrm{~mm}$ from base to tip, respectively. The array lengths of the Nucleus 24 and Nucleus 24 Contour devices are $10 \mathrm{~mm}$ and $25 \mathrm{~mm}$ (1.5 turns), respectively. The wire bundles are housed in an injection-molded silicone carrier. In contrast to the Nucleus 24 array, the Contour is pre-curled to hug the modiolus after insertion. A soft stylet wire is advanced into an opening on the back of the Contour array to straighten it pre-insertion. During insertion the array is slowly pushed off the stylet in an advance-off-stylet (AOS) technique. Cochlear Ltd. speech processors allow speech coding using CIS and variations on CIS.

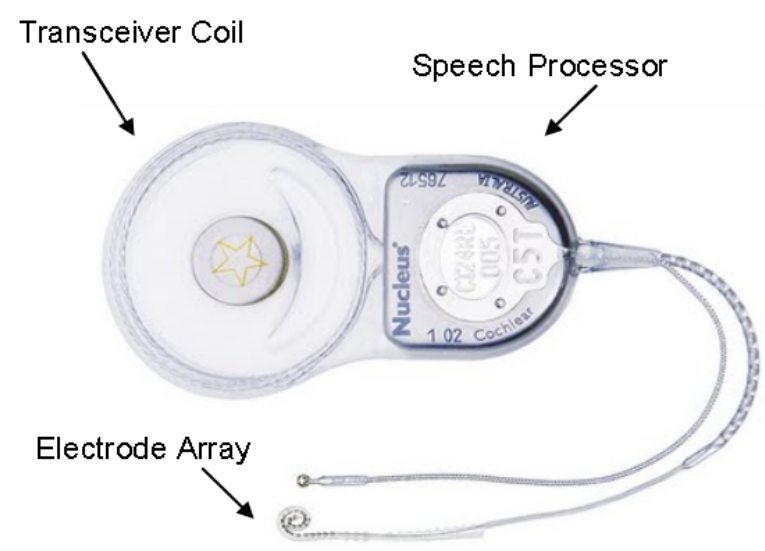

Fig. 1.7: The Cochlear Ltd. Nucleus Contour array with speech processor, transceiver coil and electrode array [29]. 
Advanced Bionics produces the HiFocus $1 \mathrm{j}$ and Helix stimulation arrays (Fig. 1.8). As with the Nucleus arrays, bundles of injection-molded wires are used, but 16 square electrode pads are used instead of bands. The $1 \mathrm{j}$ and Helix square electrodes are $0.3 \mathrm{~mm}$ on a side with center-to-center spacings of $1.1 \mathrm{~mm}$ and $0.85 \mathrm{~mm}$, respectively. The diameters of the $1 \mathrm{j}$ and Helix have a taper from base to tip of from $0.8 \mathrm{~mm}$ to $0.4 \mathrm{~mm}$ and from $0.66 \mathrm{~mm}$ to $01.16 \mathrm{~mm}$, respectively. The array length of the $1 \mathrm{j}$ and Helix arrays is $25 \mathrm{~mm}$ and $24.5 \mathrm{~mm}$, respectively. The 16 electrodes are slightly recessed into the carrier to focus the field lines. The $1 \mathrm{j}$ array has a very slight curl to it, but is mostly straight. An Electrode Insertion Tool (EIT) houses the array before insertion, and using a plunger mechanism the array is advanced into the cochlea. The Helix array is curled much like the Nucleus 24 Contour and uses a similar AOS tool for insertion. Advanced Bionics speech processors perform speech coding using CIS and variations on CA.

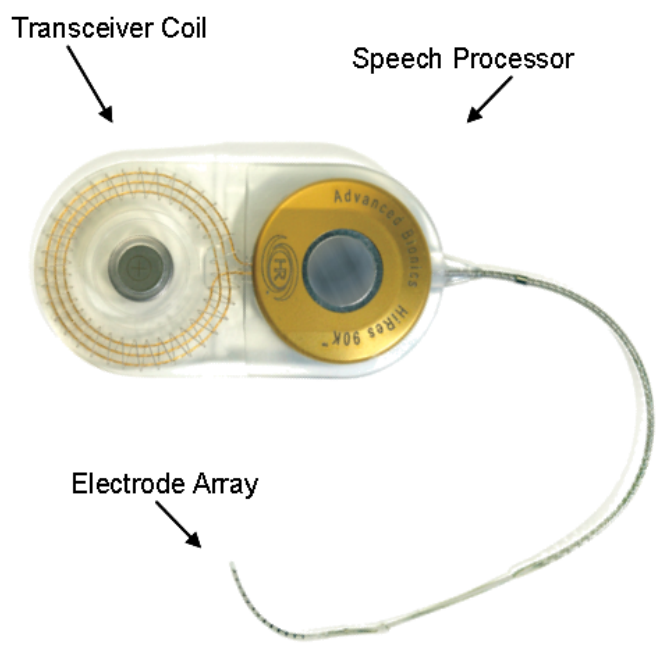

Fig. 1.8: The Advanced Bionics HiFocus array with speech processor, transceiver coil and electrode array [30].

MED-EL produces the Combi $40+$ stimulating array (Fig. 1.9). As with the Cochlear and Advanced Bionics arrays, bundles of injection molded wires are used. However, the Combi $40+$ offers a much softer and more flexible wire bundle design than its competitors. The Combi $40+$ has square electrodes, which are $0.14 \mathrm{~mm}$ on one side and are spaced $2.4 \mathrm{~mm}$ apart. The array is available in three different lengths, with the longest being $31 \mathrm{~mm}$. Shorter array designs taper from $1.33 \mathrm{~mm}$ to $0.5 \mathrm{~mm}$ from base to tip. MED-EL speech processors allow speech coding using CIS and variations on CIS. 


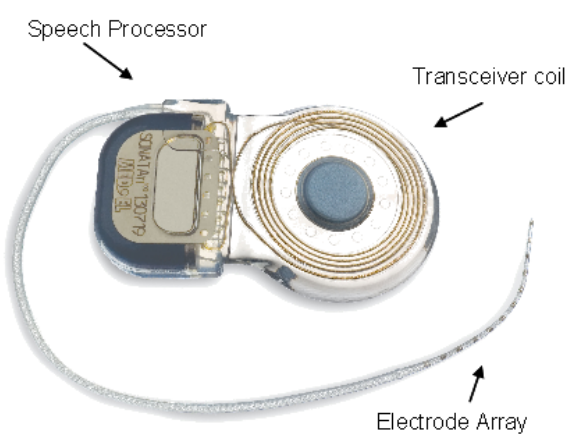

Fig. 1.9: The MED-EL Combi 40+ array with speech processor, transceiver coil and electrode array [10]

\subsection{Thin-Film Arrays}

Cochlear implants for the deaf are the most successful neural prostheses; however, pitch perception remains relatively poor. The size of the scala tympani into which the stimulating electrodes are inserted limits their number to about twenty when using conventional wires. Wire arrays are also hand assembled and relatively expensive. Thin-film arrays can offer significant advantages by increasing the number of sites (increased pitch specificity), allowing deeper insertion (greater pitch range), offering greater control over array stiffness and size, and reducing cost. However, in order for thin-film arrays to overtake wire-bundle technology they need to reach the same level of reliability and usability. To reach this level they must be robust enough for safe insertion into the helical cochlea, stiff enough for deep insertion, contain a means for placement and have a modiolus-hugging curl to position the sites close to receptor cells and reduce insertion trauma. To date, thin-film arrays have been fabricated by depositing metal leads and sites on inorganic dielectrics and silicon, sandwiching them between two polymer substrates, or a combination of both. A review of past thin-film technologies is given below along with the disadvantages and advantages of each design.

\subsubsection{Polymer Arrays}

The first thin-film cochlear array to be developed was comprised of polymermetal-polymer sandwiched layers [31]. The probes had 36mm long shanks containing 
$16,250 \mu \mathrm{m}$ diameter electrodes, spaced $700 \mu \mathrm{m}$ apart. They were comprised of sputtered platinum (Pt) sandwiched between $50 \mu \mathrm{m}$ thick Mylar sheets. A silicone casing was used for added robustness and was selectively removed with a blade. Although, these arrays were fabricated using a combination of thin-film and hand assembly techniques, they initiated a move away from wire-bundle technology. The next polymer probes came out of Melbourne [32] and were made of Pt sputtered over $120 \mu \mathrm{m}$ of fluorinated ethylenepropylene (FEP) Teflon, and insulated with polytetrafluoroethylene (PTFE) Teflon which was selectively removed using a liftoff technique. In contrast to the Mylar arrays, the Teflon devices were fabricated with more automated methods, such as the use of liftoff versus blade cutting to remove the insulation. However, both technologies relied on the deposition and etching of Pt over thin polymer sheets, which could be a tedious and unreliable process [31]. Stanford produced the first non-polymer sheet arrays, using the polyimide composite Pyralin (PI-2555) [33]. The arrays were fabricated by spin coating polyimide $(50-150 \mu \mathrm{m})$ on silicon carriers and then sputter-coating tantalum $(\mathrm{Ta}) / \mathrm{Pt}$ for the lead lines and electrode sites. Ta served as an adhesion promoter between the Pt and the polyimide. A top insulating layer of polyimide $(10 \mu \mathrm{m})$ was used to seal the array. Although spin-coated polyimide provided better metal adhesion and ease of processing than the polymer sheets, it has been shown to degrade in fluid environments over time [34].

All of the previously mentioned polymer arrays relied on substrate thickness as a means of insertion; however, it is difficult to make such thin materials stiff enough for insertion without buckling. Additionally, no provision was made in any of the designs for array curvature. The issue of curvature was addressed by Wu, et al. [35], through the addition of polypyrrole (Ppy) to their polymer-metal-polymer stack. Ppy is a conducting polymer that undergoes volume changes in response to applied potentials. The polymer was deposited on an SU-8 and gold ( $\mathrm{Au}$ ) array, which was subsequently pre-curved. To straighten the array, a potential is applied across the Ppy to expand its volume and uncurl the array. Near straight array profiles were achieved using Ppy, but no mention is made of how a thin film array would be backed to achieve insertion. In an effort to address the concerns of both backing and curl, Corbett, et al., of Advanced Cochlear Systems developed a thin-film array by stacking Liquid Crystal Polymer (LCP) laminates [36]. 
The $25 \mu \mathrm{m}$ layers of LCP were each sputter-deposited with chromium-gold lead lines and then stacked in 7 layers to form a complete 12-electrode array (Fig. 1.10). The finished array was curled by heat-forming it in a steel mold [37]. Initial results of this array showed significant leakage of the LCP laminate layers, which may not make it suitable for long term use.
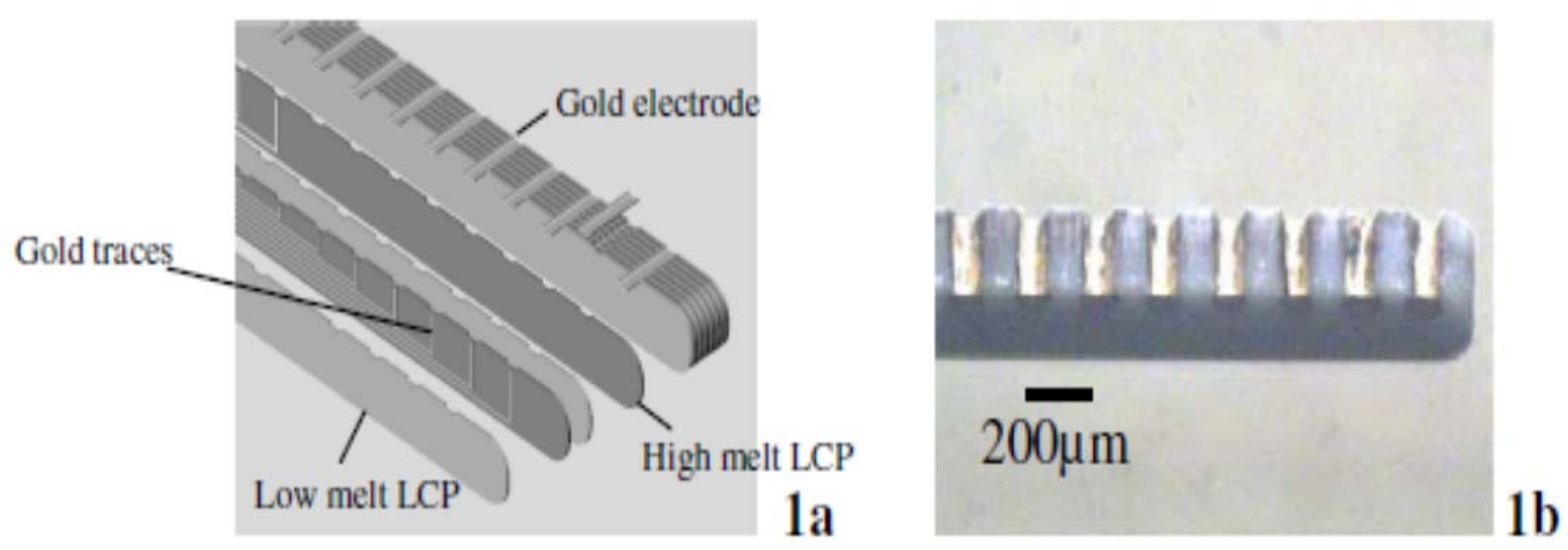

Fig. 1.10: The Advanced Cochlear Systems arrays comprised of stacked Aupatterned LCP layers [36].

\subsubsection{Silicon Arrays}

The first silicon thin-film cochlear electrode arrays were developed by Bell and Wise at the University of Michigan [38]. The arrays consisted of 22 iridium (Ir) oxide electrodes and attached polysilicon lead lines over boron-doped silicon substrates. The electrodes were $250 \mu \mathrm{m}$ in diameter with center-to-center spacings of $750 \mu \mathrm{m}$. They were made of activated iridium oxide ( $\mathrm{IrO}$ ) as opposed to $\mathrm{Pt}$, since it had been shown to safely deliver higher charge densities [39], which can be high in such small-area sites. Boron was diffused to two separate depths in the silicon as an etch stop for creating $4 \mu \mathrm{m}$ and $12 \mu \mathrm{m}$-thick regions. The $4 \mu \mathrm{m}$ regions formed the intracochlear shanks, which tapered from a width of $640 \mu \mathrm{m}$ at the base to a width of $320 \mu \mathrm{m}$ at the apex. The $12 \mu \mathrm{m}$ regions provided a stiffer substrate for bonding to Printed Circuit Boards (PCBs). To insulate the trace lines, stress-compensated oxide-nitride-oxide dielectric stacks were used. The silicon arrays were able to initiate auditory brain responses in guinea pigs through the cochlea [38]. Based on the initial success of these first-generation passive arrays, Bell and Wise went on to create a second generation of active arrays [40]. These 
active arrays contained decoders and switches along the probe shank for site selection, and shift registers and latches at the backend to feed in command words. They were designed to work in conjunction with site selection circuitry placed along the shank of the array to reduce the number of lead lines, and only four bus lines were routed to the backend of the array. The lead count was reduced so that the bus lines could be made wider to lower voltage drops from the probe sites to the backend without having to add additional metal layers. The circuitry was fabricated using a p-well $3 \mu \mathrm{m}$ CMOS process developed at the University of Michigan. In addition to the stimulation circuitry, position sensors were added to view array position inside the cochlea. The sensors were eight arsenic-doped polysilicon strain gauges connected in series going down the probe shank whose changes in resistance due to bending [40] were referenced to a resistor at the backend of the array. These active array features were implemented on the cochlear arrays, but never fully tested due to difficulties in fabrication. However, both of these active array features were further modified and realized in third generation arrays developed at the University of Michigan [41].

Bhatti and Wise designed an active, four-channel, 32-site silicon cochlear array sized for a guinea pig cochlea $[42,43]$. The silicon arrays were fabricated using the same process as the Bell arrays, with $2.5 \mu \mathrm{m}$-thick shanks, and $14 \mu \mathrm{m}$-thick backends. The IrO electrodes were $180 \mu \mathrm{m}$ in diameter and spaced $250 \mu \mathrm{m}$ center-to-center down a $8 \mathrm{~mm}$ probe shank which tapered from $500 \mu \mathrm{m}$ to $200 \mu \mathrm{m}$, base to apex (Fig. 1.11). Bhatti increased the functionality of her active arrays by adding circuitry for current generators and error checking. Additionally, position sensing circuitry was also added to the active array in collaboration with work done by Wang and Wise [44]. 


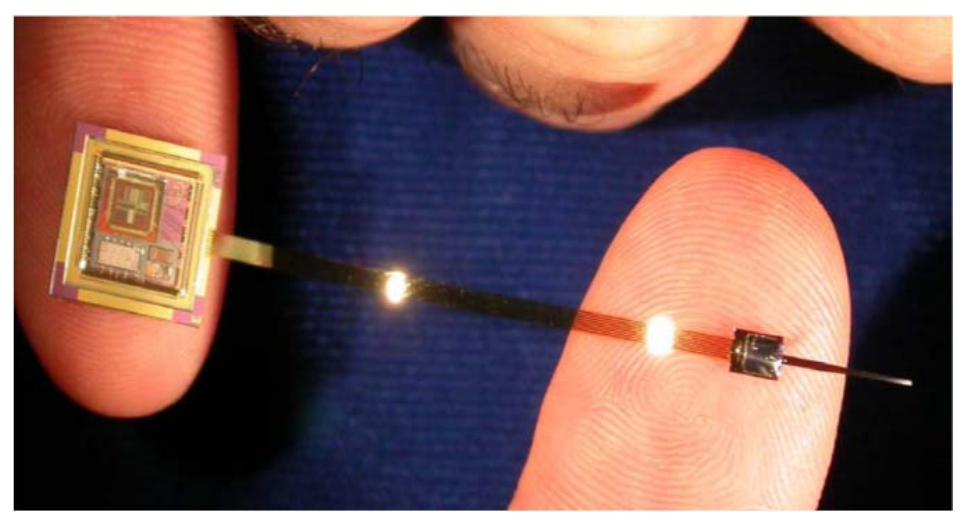

Fig. 1.11: The Michigan cochlear electrode array integrated with a polyimide cable to the ASIC and microcontroller. [42]

With this additional functionality it became evident that the distributed $3 \mu \mathrm{m}$ BiCMOS process used by Bell would be too large for the size constraints of the cochlea [42]. Thus, Bhatti opted for the placement of an Application Specific Integrated Circuit (ASIC) at the backend of the array using a $0.5 \mu \mathrm{m}$ AMI CMOS process. This ASIC was capable of biphasic stimulation levels up to $500 \mu \mathrm{A}$ using monopolar, bipolar, and tripolar configurations. It operated from a $\pm 2.5 \mathrm{~V}$ supply voltages, consumed $2.5 \mathrm{~mW}$ at $5 \mathrm{~V}$, and had a total area of $2.4 \mathrm{~mm} \times 2.9 \mathrm{~mm}$.

The silicon cochlear arrays were able to provide much more functionality than had been done before. However, equipping the devices with insertion and modiolus hugging mechanisms was challenging. In collaboration with Michigan Technological University, precurled polyethylene therephthalate (PET) backing tubes were attached to the backs of the arrays, so that a pneumatic insertion technique could be utilized. The $200 \mu \mathrm{m}$ wide PET tubing had multiple chambers, where each chamber controlled a separate segment of the array [45]. With this type of design, fluid could be flowed through each of the chambers to uncurl the array in an articulated actuation scheme. While some success was had with this backing method, mismatches in stiffness between the silicon and PET often led to poor insertion [44]. Alternate backing methods included applying a silastic carrier to the back of the silicon arrays to increase their robustness and provide a space-filling mechanism to push them against the modiolus after insertion. However, the silicone molding was hard to apply to the arrays without breaking them and only slightly improved survival during insertion. To increase the flexibility of the array 
substrate, Wang et al, suggest removing portions of the silicon substrate to create siliconpolymer-dielectric hybrid arrays [44]

\subsubsection{Silicon-Polymer Hybrid Arrays}

In efforts to overcome the issues of silicon fracturing during insertion, Wang et al., produced a silicon-parylene-inorganic-dielectric substrate cochlear electrode array [46]. The array was fabricated in much the same way as the previous generation arrays from Michigan, containing all the same dimensional attributes. What was different in this new cochlear electrode array was the substrate composition (Fig. 1.12).

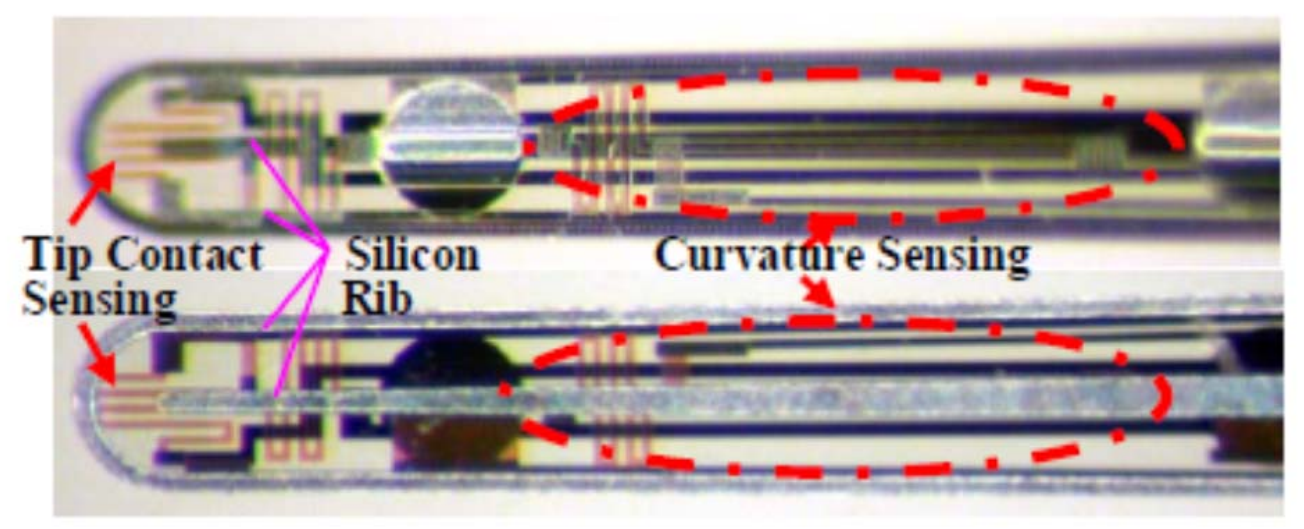

Fig. 1.12: A silicon - parylene - inorganic dielectric thin-film array with IrO sites for stimulation and piezoresistive strain gauges for position sensing [46].

A dielectric stack of oxide-nitride-oxide provides the main support layer for the array, upon which polysilicon strain gauges and $\mathrm{IrO}$ electrodes sites were built. Surrounding the dielectric substrate was a silicon frame and a center rib for stiffness. To increase the flexibility and robustness of the array, parylene was deposited over the dielectric and silicon. Reducing the amount of silicon in the substrate from total coverage to a rib increased the flexibility of the arrays by an order of magnitude [46]. Not much improvement in flexibility was achieved with the addition of parylene, since it was merely an overlay on a stiffer dielectric and silicon substrate. The arrays were still susceptible to fracture during insertion, and the parylene merely served as a means of keeping the broken substrate pieces together in case of fracture [46]. 
Taking the concept of a hybrid silicon-polymer array a step further, Bulcke, et al., made polymer materials the primary substrate layer for the arrays and used silicon only in the electrode areas [47]. Additionally, they revisited the early concept of a distributed circuits along the shank of the probe propose by Bell and Wise [38]. Their process begins with the thinning down of a CMOS wafer containing a multiplexing circuit block for site selection. The wafer is thinned by first grinding down the backside to $50 \mu \mathrm{m}$ and doing a Reactive Ion Etch (RIE) to thin it to a final thickness of $15 \mu \mathrm{m}$ [48]. This thinned CMOS wafer is mounted face down on a carrier and then aligned and bonded using benzocyclobutene $(\mathrm{BCB})$ to another wafer which has a corresponding array of $\mathrm{Pt}$ electrodes. $\mathrm{BCB}$ is used for bonding, and copper $(\mathrm{Cu})$ feed throughs are used to connect the stacked wafers with an Ultra Thin Chip Stacking (UTCS) process developed by IMEC [48]. After stacking, the active electrode units are singulated and encapsulated in Pt. Next, the units are connected with Pt bus lines and sealed in parylene (Fig. 1.13). The entire array is fully encapsulated with a photo-patternable silicone.

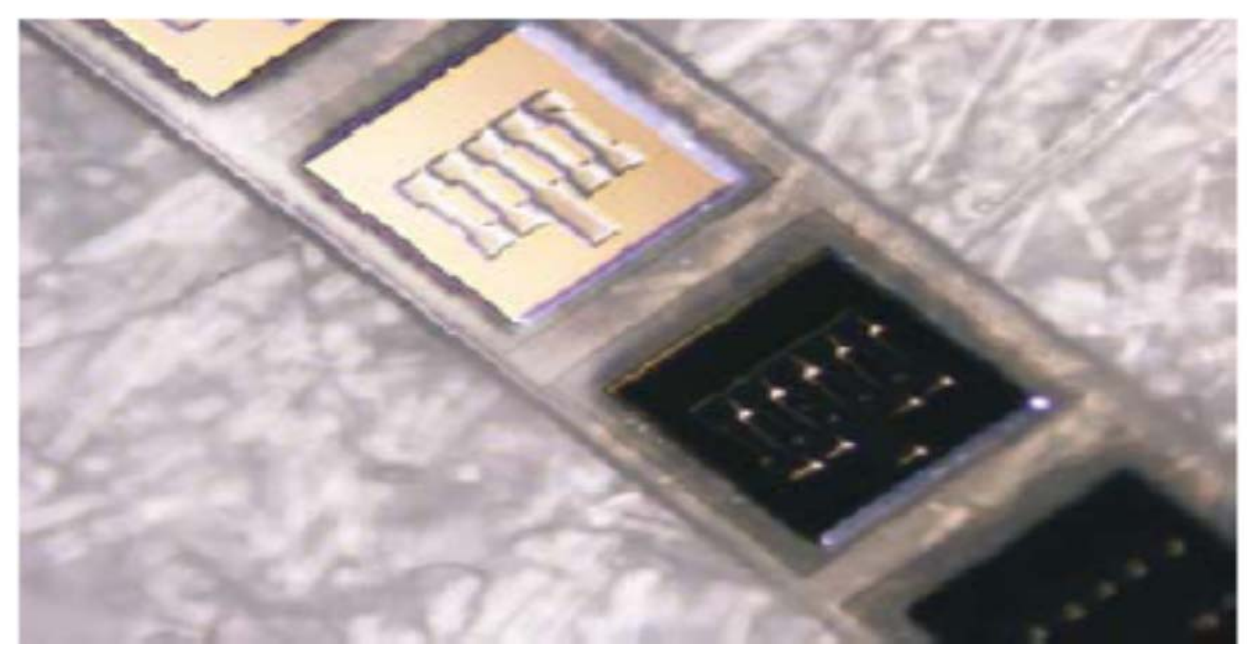

Fig. 1.13: The IMEC active electrode array with silicon islands encased in silicone [ref].

This active electrode technology allowed for the creation of a 22-site array with only five bus lines extending down the array shank (clock, power, ground, signal and address). Additionally, the silicone and parylene polymer layers made the array highly flexible. However, the photo patternable silicone is not a proven biocompatible material, and the deposition and patterning of medical grade silicone at wafer level is difficult [49]. 
Further, interelectrode step heights are likely due to the non-uniform RIE process. Lastly, no provisions were made for array insertion or modiolus-hugging features.

\subsubsection{Summary of Thin-Film Arrays}

A variety of designs have been reported for thin-film cochlear electrode arrays since the early 1980s. From the beginning, biocompatible polymer materials were thought to be excellent substrates for the arrays because of their flexibility. Later, silicon substrates were explored because of well characterized bulk silicon fabrication techniques and the ability to define smaller feature sizes on high quality dielectric insulators. Due to the stiffness of silicon substrates, however, it became evident that the partial incorporation of polymer materials was advantageous. The inclusion of electronics as necessary components of the arrays also developed over time in an effort to move to a fully implantable system and reduce lead resistances for high density arrays. Both distributed circuitry local to the electrode sites and centralized backend circuitry schemes were investigated. As advances were made in the development of the cochlear stimulating arrays and complementary electronics, more consideration was given to how the thin-film arrays could be inserted into the helical cochlea and positioned to hug the modiolus. Traditionally, insertion has been done using stylet wires, plungers, or direct placement techniques, and modiolus hugging has been achieved through space fillers and pre-curving the arrays. To achieve the same goals, thin film arrays were designed with increased substrate thicknesses in an effort to use direct placement and with multichamber tubes in an effort to use pneumatically actuated insertion. Space-filling silicone moldings, curved backings, and curved arrays were used in an effort to hug the modiolus. 
Table 1.1: Comparison of Three Thin-Film Cochlear Electrode Array Technologies.

\begin{tabular}{|c|c|c|c|}
\hline \multicolumn{4}{|c|}{ Thin-Film Cochlear Array Comparison } \\
\hline Device & & & \\
\hline Developer & $\begin{array}{l}\text { Advanced Cochlear } \\
\text { Systems }\end{array}$ & $\begin{array}{l}\text { University of } \\
\text { Michigan }\end{array}$ & $\begin{array}{l}\text { Interuniversity } \\
\text { Microelectronics } \\
\text { Centre (IMEC) }\end{array}$ \\
\hline Materials & $\begin{array}{l}\text { Substrate: } \mathrm{LCP} \\
\text { Traces: } \mathrm{Cr} / \mathrm{Au} \\
\text { Electrodes: IrO }\end{array}$ & $\begin{array}{l}\text { Substrate: Silicon, } \\
\text { Oxide-Nitride, } \\
\text { Parylene } \\
\text { Traces: Aluminum } \\
\text { Electrodes: IrO }\end{array}$ & $\begin{array}{l}\text { Substrate: Silicon, } \\
\text { Silicone, Parylene } \\
\text { Traces: Pt } \\
\text { Electrodes: Ti/Al }\end{array}$ \\
\hline $\begin{array}{l}\text { Young's Modulus } \\
\text { of Flex Limiting } \\
\text { Substrate }\end{array}$ & LCP: 9 -19GPa & Si: $169 \mathrm{GPa}$ & $\begin{array}{l}\text { Silicone: } 13- \\
21 \mathrm{GPa}\end{array}$ \\
\hline \# Electrodes & 72 & 128 & Not published \\
\hline Insertion Method & Direct placement & $\begin{array}{l}\text { Pneumatically } \\
\text { actuated PET tubing }\end{array}$ & Direct Placement \\
\hline $\begin{array}{l}\text { Modiolus Hugging } \\
\text { Features }\end{array}$ & $\begin{array}{l}\text { Substrate thickness } \\
\text { used as space filler }\end{array}$ & $\begin{array}{l}\text { Adhesively attached } \\
\text { Curled PET tubing }\end{array}$ & None \\
\hline Electronics & None & $\begin{array}{l}\text { 4-channel, ASIC } \\
\text { with stimulation \& } \\
\text { recording modes }\end{array}$ & $\begin{array}{l}\text { Distributed circuitry } \\
\text { on array shank, with } \\
\text { active electrode }\end{array}$ \\
\hline
\end{tabular}

\subsection{Thesis Objectives}

Previous-generation Michigan cochlear arrays have demonstrated the ability to produce high-density arrays with integrated position sensors and local stimulation circuitry. They were a major advance in the realization of a complete thin-film cochlear prosthetic system and have taken the technology further than other efforts in this area. The goal of the research reported here was to improve the reliability, usability and functionality of the previous-generation arrays.

The main objective of this thesis was to redesign the active cochlear electrode array to be more robust while adding monolithically-fabricated backing and curl structures and increasing the functionality of the cochlear ASIC.

A robust parylene-substrate array has been created which contains a residuallystressed, self-curling parylene-metal-parylene substrate and a parylene backing channel 
compatible with stylet wire insertion methods. The monolithic bimorph and backing channel will serve to eliminate to the messy and inefficient application of array backings post-fabrication. These structures also give greater control of array stiffness and curvature.

An update of the previous-generation ASIC was done to improve system performance and expand available stimulation configurations. It has added functionality to push the frequency-selectivity limits of multipolar site configurations and current shaping strategies.

Chapter 2 of this report describes the design and fabrication of non-backed parylene cochlear electrode arrays. Chapter 3 describes the addition of molded and thin-film backings to these flexible parylene arrays, which allow them to be inserted and positioned close to the modiolus wall. Particular emphasis is placed on the development of monolithic backing structures since these backings are best suited to the realization of a thin-film cochlear prosthetic. Chapter 4 discusses in-vivo animal implants that were conducted to assess the performance of the thin-film arrays. Both cat and guinea pig, acute animal studies were tested. Chapter 5 reviews the functionality and performance of a next-generation cochlear ASIC. Lastly, Chapter 6 summarizes the significant contributions of this thesis and proposes future research goals.

\subsection{References}

[1] Cavuoto, J. , "The Market for Neurotechnology: 2008-2012," Neurotech Reports, 2007.

[2] Hawkins, J., "Human Ear, ” Encyclopæedia Britannica Online Academic Edition, Aug. 2011, http://www.britannica.com/EBchecked/topic/175622/ear.

[3] Mather, G., Foundations of perception, 2006.

[4] "Hearing and Hair Cells", Stanford School of Medicine Oghalalai Lab, Aug. 2011, http://oghalailab.stanford.edu/hearing_haircell.html

[5] Rose, J. E. , "Phase-locked response to low-frequency tones in single auditory nerve fibers of the squirrel monkey," Journal of neurophysiology, vol. 30, pp. 769, 1967. 
[6] Nomura, Y. "Nerve fibers in the human organ of Corti," Acta oto-laryngologica, vol. 82, pp. 317, 1976.

[7] Clark, G., "Temporal coding of frequency: neuron firing probabilities for acoustic and electric stimulation of the auditory nerve." The annals of otology, rhinology \& laryngology. Supplement, vol. 166, pp. 109-111, 1995.

[8] Kiang, N. Y.-S., "Stimulus coding in the auditory nerve and cochlear nucleus," Acta oto-laryngologica, vol. 59, pp. 186, 1965.

[9] “...And Ears to Hear...”, Teddy’s Rat Lab, Aug. 2011, http://teddysratlab.blogspot.com/2011/03/and-ears-to-hear.html

[10] MED EL, Aug. 2011, http://www.medel.com

[11] Eddington, D. K., "Speech discrimination in deaf subjects with cochlear implants," The Journal of the Acoustical Society of America, vol. 65, pp. S103, 1979.

[12] Eddington, D. K., "Speech recognition in deaf subjects with multichannel intracochlear electrodes," Annals of the New York Academy of Sciences, vol. 405, pp. 241, 1983.

[13] Coope, H.; Craddock, L., Cochlear Implants: A Practical Guide, 2nd Edition, 2 ed.: Wiley, 2006.

[14] Boëx, C., "Electrical field interactions in different cochlear implant systems," The Journal of the Acoustical Society of America, vol. 114, pp. 2049, 2003.

[15] Wilson, B. S. ; Finley, C. C. Lawson, ; D. T.; Wolford, R. D., Eddington, D. K.; Rabinowitz, W. M., "Better speech recognition with cochlear implants," Nature, vol. 352, pp. 236-238, 1991.

[16] Loizou, P. C., "Mimicking the human ear," IEEE signal processing magazine, vol. 15, pp. 101, 1998.

[17] Clark, G., Cochlear implants : fundamentals and applications new york: Springer, 2003.

[18] Chen, B., "Evaluation of trajectories and contact pressures for the straight nucleus cochlear implant electrode array - a two-dimensional application of finite element analysis," Medical engineering \& physics, vol. 25, pp. 141, 2003.

[19] Kha, H.N. "Stiffness properties for Nucleus standard straight and contour electrode arrays," Medical engineering \& physics, vol. 26, pp. 677, 2004. 
[20] Wardrop, P. ; Whinney, D. ; Rebscher, S. J.; Roland, J. T.; Luxford, W.; Leake, P. A., "A temporal bone study of insertion trauma and intracochlear position of cochlear implant electrodes. I: Comparison of Nucleus banded and Nucleus Contour ${ }^{\mathrm{TM}}$ electrodes," Hearing Research, vol. 203, pp. 54, 2005.

[21] Suesserman, M. F.; Spelman, F. A., "Lumped-parameter model for in vivo cochlear stimulation," IEEE transactions on bio-medical engineering, vol. 40, pp. $237,1993$.

[22] Townshend, B. , "Pitch perception by cochlear implant subjects," The Journal of the Acoustical Society of America, vol. 82, pp. 106, 1987.

[23] Snyder, R. L., "Cochlear implant electrode configuration effects on activation threshold and tonotopic selectivity," Hearing Research, vol. 235, pp. 23, 2008.

[24] Hochmair-Desoyer, I. J. ; Hochmair, E. S.; Burian, K., "Design and fabrication of multiwire scala tympani electrodes," Annals of the New York Academy of Sciences, vol. 405, pp. 173, 1983.

[25] Van Den Honert, C.; Stypulkowski, P. H., "Single fiber mapping of spatial excitation patterns in the electrically stimulated auditory nerve," Hearing Research, vol. 29, pp. 195-206, 1987.

[26] Donaldson, G. S. ; Kreft, H. A. ;. Litvak,L., "Place-pitch discrimination of singleversus dual-electrode stimuli by cochlear implant users," The Journal of the Acoustical Society of America, vol. 118, pp. 623, 2005.

[27] Advanced Bionics Corporation, "Increasing spectral channels through current steering in HiResolution bionics ear users," Aug. 2011, http://www.advancedbionics.com

[28] HONET, C., "Pitch Steering with Sequential Stimulation of Intracochlear Electrodes.," Cochlear research report, Cochlear USA, 2006.

[29] Cochlear Americas, Aug. 2011, http://www.cochlearamericas.com

[30] Advanced Bionics Corporation, Aug. 2011, www.advancedbionics.com

[31] Sonn, M.; Feist, W.M., "A prototype flexible microelectrode array for implantprosthesis applications," Medical \& biological engineering, vol. 12, pp. 778, 1974.

[32] Clark, G. M.; Hallworth, R.J.,"A multiple-electrode array for a cochlear implant," The Journal of Laryngology and Otology, vol. 90, pp. 623, 1976. 
[33] Shamma-Donoghue, S. A.; May, G. A.; Cotter, N. E.; White, R. L.; Simmons, F. B. , "Thin-film multielectrode arrays for a cochlear prosthesis," IEEE transactions on electron devices, vol. 29, pp. 136, 1982.

[34] Anderson, D. J.; Najafi, K.; Tanghe, S. J.; Evans, D. A.; Levy, K. L.; Hetke, J. F.; Xue, X. ; Zappia, J. J.; Wise, K. D., "Batch fabricated thin-film electrodes for stimulation of the central auditory system," IEEE transactions on bio-medical engineering, vol. 36, pp. 693, 1989.

[35] Jian Wu; Le Yan; Han Xu; Tang, W.C.; Fan-Gang Zeng; , "A curvature-controlled 3D micro-electrode array for cochlear implants," The 13th International Conference on Solid-State Sensors, Actuators and Microsystems, vol.2, pp. 16361639,2005

[36] Corbett, S.; Ketterl, J.; Johnson, T., "Polymer-Based Microelectrode Arrays," Mater. Res. Soc. Symp. Proc., vol. 926, pp. 0926-CC06-02, 2006.

[37] S. Corbett, T. Johnson, B. Clopton, F. Spelman, J. Strole, and J. Ketterl, "Method of making high contact density electrode array." vol. 6782619, 2004.

[38] Bell, T. E. , "A flexible micromachined electrode array for a cochlear prosthesis," Sensors and actuators. A, Physical, vol. 66, pp. 63, 1998.

[39] Weiland, J. D. , "Electrochemical properties of iridium oxide stimulating electrodes," in Biomedical Engineering, Ph.D. Ann Arbor: The University of Michigan, 1997, pp. 128.

[40] Bell, T. , "A cochlear implant fabricated using a bulk silicon-surface micromachining process," in Electrical Engineering and Computer Science, Ph.D. Ann Arbor: The University of Michigan, 1999.

[41] Wang, J.; Wise, K. D. "High-density cochlear implants with position sensing and control," Hearing Research, vol. 242, pp. 22, 2008.

[42] Bhatti, P.T. , "A high-density thin-film electrode array for a cochlear prosthesis," in Electrical Engineering and Computer Science, Ph.D. Ann Arbor: The University of Michigan, 2006.

[43] Bhatti, P. T. ; Wise, K. D. , "A 32-site 4-channel high-density electrode array for a cochlear prosthesis," IEEE journal of solid-state circuits, vol. 41, pp. 2965, 2006.

[44] Wang, J.; Wise, K.D., “A cochlear electrode array with built-in position sensing”, 18th IEEE International Conference on Micro Electro Mechanical Systems, pp. 786-789, 2005. 
[45] Arcand, B. Y. ; Bhatti, P. T.; Butala, N. V.; Friedrich, C. R. ; Wise, K. D. , "Active positioning device for a perimodiolar cochlear electrode array," Microsystem Technologies, vol. 10, pp. 478 - 483, 2004.

[46] Wang, J.; Gulari, M. N. ; Wise, K. D., "A Parylene-Silicon Cochlear Electrode Array with Integrated Position Sensors," 28th IEEE International Conference on Engineering in Medicine and Biology Society, pp. 3170-3173, 2006

[47] Bulcke, M. V., Baert, K.; Beyne, E.; Gonzalez, M.; Winters, C.; Webers, T., "Active Electrode Arrays by Chip Embedding in a Flexible Silicone Carrier," 28th IEEE International Conference on Engineering in Medicine and Biology Society, pp. 2811-2815, 2006

[48] Vendier, O. , "Ultra thin electronics for space applications", 51st Electronic Components and Technology Conference, pp. 767 - 761, 2001.

[49] Schuettler, M. ; Stiess, S.; King, B. V.; Suaning, G. J., "Fabrication of implantable microelectrode arrays by laser cutting of silicone rubber and platinum foil," Journal of neural engineering, vol. 2, pp. S121 - S128, 2005. 
Chapter 2

\section{DESIGN AND FABRICATION OF A PARYLENE COCHLEAR ELECTRODE ARRAY}

A cochlear electrode array needs to be flexible enough for intracochlear insertion, contain modiolus hugging features for close proximity of the electrode sites to the ganglion cells, and be compatible with appropriate insertion techniques. Addressing all of these concerns using a thin-film approach requires the proper selection device dimensions, substrate materials and MEMS processing techniques. This chapter focuses on the design and fabrication of a non-backed parylene cochlear electrode array. It begins with a discussion of the design of the parylene arrays in relation to device dimensions and materials. Next, the array fabrication steps are outlined and a closer look is taken into the fabrication challenges and considerations. Lastly, an examination of the mechanical and electrical in-vitro performance of the finished arrays will be presented.

\subsection{Array Materials}

In the past, cochlear arrays were composed primarily of silicon, inorganic dielectrics and metal layers. These arrays performed well electrically; however, due to

their brittle nature, they were prone to breaking during in-vitro tests [1], and they could not be inserted very far into the cochlear without breaking [2]. The focus of this work, was to create a thin-film cochlear array that could survive full insertion into the cochlea, yet still maintain good electrical performance. The following sections review the critical material selections for array mechanical and electrical performance.

\subsubsection{Parylene-C Substrate}

In choosing an array substrate material, considerations were given to biocompatibility, flexibility (Young's modulus, elongation to break) and processing ease. 
Polydimethylsiloxane (PDMS), polyimide, SU-8, and parylene C were considered as potential substrate materials (Table 2.1). SU-8 is not a brittle material like silicon and has a low Young's modulus (2Gpa); however, it has a very low elongation to break ( $<$ $5 \%$ ) and tends to break under in-plane flexing. PDMS and polyimide are better able to handle in-plane flexing, with elongations to break of less than $40 \%$ and $30 \%$, but their photopatternable formulations are not safe for long-term implantation in the body. Similar medical-grade silicones exist, but etching of these materials typically requires laser ablation and so processing with them can be difficult. In addition to the difficulty of removal, silicone is also is highly absorbent to solvents, and swelling further increases the processing complexities when attempting to do define fine $(\leq 10 \mu \mathrm{m})$ line pitches using acetone liftoff. Parylene provides a good alternative to the aforementioned materials due to its high elongation to break (200\%), favorable FDA approval status for long term implants (USP class VI), pinhole-free vapor deposition, and easy etching in $\mathrm{O}_{2}$ plasmas.

Table 2.1 Substrate Material Comparison.

\begin{tabular}{|c|c|c|c|}
\hline Material & $\begin{array}{c}\text { Implantable } \\
\text { long-term }\end{array}$ & $\begin{array}{c}\text { Young's Modulus } \\
\text { (GPa) }\end{array}$ & $\begin{array}{c}\text { Elongation at } \\
\text { break }\end{array}$ \\
\hline Boron-doped Silicon & Yes & 185 & $0 \%$ \\
\hline $\begin{array}{c}\text { Photopatternable PDMS } \\
\begin{array}{c}\text { Photopatternable } \\
\text { Polyimide }\end{array}\end{array}$ & No & 0.16 & $<40 \%$ \\
\hline SU-8 & No & 3.8 & $<20 \%$ \\
\hline Parylene C & Yes & 2 & $<5 \%$ \\
\hline
\end{tabular}

Parylene is one of a family of poly-para-xylylene polymers. Its chemical compound contains two hydrogen atoms and one chlorine (Fig. 2.1). This inert structure makes it a good barrier against most gases and liquids with a gas permeability to oxygen of $3.0 \mathrm{cc}-\mathrm{mm} /\left(\mathrm{m}^{2} \cdot\right.$ day.atm $)$ and a moisture vapor transmission rate of just 0.08 $\mathrm{g} \cdot \mathrm{mm} / 100 \mathrm{~m}^{2} \cdot$ day [3]. Additionally the material has good dielectric properties with a dielectric constant of 2.95 at $1 \mathrm{MHz}$. Parylene $\mathrm{C}$ is deposited through a vapor deposition process which makes coating with the material pin-hole free and conformal. All of these 
features combined with the room temperature deposition process have resulted in the material being used in a range of applications for the coating of medical implants, electronics and mechanical structures [5,6,and 7]. Parylene can be deposited with pinhole-free vapor deposition in thicknesses ranging from nanometers to microns.

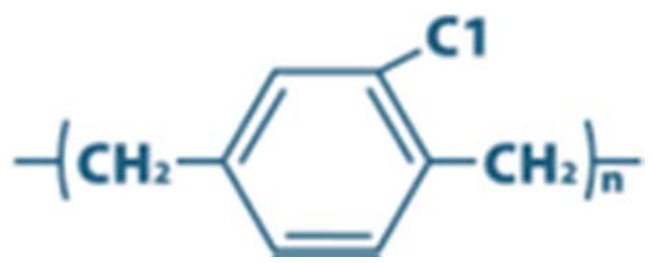

Fig. 2.1: Molecular structure of Parylene C [4]

The deposition process for Parylene C occurs in three stages[8]: (1) a parylene C dimer di-para-xylylene is vaporized to a gaseous phase by elevated heating to $\sim 175^{\circ} \mathrm{C}$ at 1 Torr (2). The gaseous dimmer is split into para-xylylene monomers at $680^{\circ} \mathrm{C}$ in 0.5 Torr (3), and the monomers polymerize at room temperature onto surfaces under a pressure of less than 0.1Torr (Fig. 2.2).

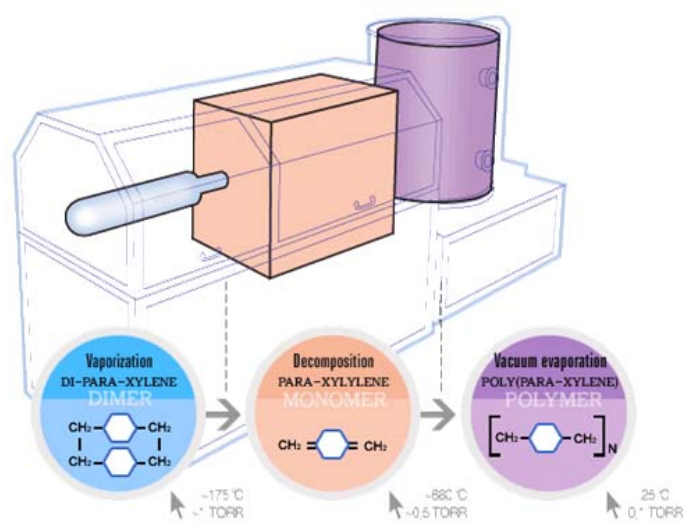

Fig. 2.2: The Parylene- $\mathrm{C}$ deposition process [8].

\subsubsection{Iridium Oxide Electrode Sites}

When stimulating cells with an electrode, charge is created in the electrolytic medium through the capacitive Helmholtz double layer and through the Faradaic resistance created through reversible oxidation and reduction reactions. As such, the 
electrical model for stimulation has both a capacitive and resistive component. Potentials applied to the electrode are what initiate and reverse these capacitive and resistive charge creations. However, when potentials are too great it creates hydrolysis, which exerts physical and chemical damage to the surrounding cells. In order to be used for biostimulation, the electrode metals need to be inert (do not corrode under current pulsing), biocompatible, and have high charge capacities.

Common electrode materials include platinum (Pt), Iridium (Ir) platinum black(Pt black) and Iridium Oxide (IrO). When choosing among these four materials, the characteristics of greatest interest to cochlear electrode sites are the site impedance and charge capacity. This is because cochlear prostheses use high current stimulation amplitudes of up to $1 \mathrm{~mA}$. Studies have shown that IrO sites have lower impedance and greater charge densities than any of the other three alternatives [9]. Typically charge densities for platinum are in the $0.4 \mathrm{mC} / \mathrm{cm}^{2}$ in comparison to $30 \mathrm{mC} / \mathrm{cm}^{2}$ for $\mathrm{IrO}$ sites [10]. The increased charge capacity is a result of the increased surface areas provided by the addition of porous oxide. One way of creating such sites is through Activated Iridium Oxide Films (AIROFs). The films are created by growing a porous layer of oxide over Ir substrates with Cyclic Voltametry (CV). When Ir is cycled between positive and negative potentials (below the water window) oxidation and reduction reactions build an oxide on the surface. As the potential initially sweeps positive, high oxidation reactions create first a layer of $\mathrm{IrO}_{2}$, with subsequent increases in potential forming a second layer of $\mathrm{IrO}_{3}$. This second layer is hydrolyzed by surrounding water molecules to form $\operatorname{Ir}(\mathrm{OH})_{3}$. When the potential sweeps negative, it reverses the oxidation of $\mathrm{IrO}_{2}$, but the hydrous oxide remains. Since the water molecules surround $\operatorname{Ir}(\mathrm{OH})_{3}$ it does not form a monolayer, and this produces the highly porous nature of the oxide coating. Parameters such a waveform shape, potential bounds, and number of cycles all factor into the quality and amount of the oxide.

For the cochlear electrode arrays presented in this work, charge capacity is of special concern, given the high biphasic stimulation amplitudes of $\pm 500 \mu \mathrm{A}$. As such, IrO sites are used in all array designs. The sites are created with sputtered Ir that has been activated with Cyclic Voltammetry parameters similar to those outlined by Weiland [10]. The process of fabrication and results are covered in the following sections. 


\subsection{Array Dimensions}

To test the fabrication of and performance of a full-scale human cochlear array, guinea pig (GP) and cat array prototypes were developed. Both animal models are 32site scaled-down versions of a 128-site human array. These two particular animals were chosen because they are commonly used in cochlear implant studies. The guinea pig is a more affordable and easier-to-access model, while the cat cochlea is closer in size and scale to a human cochlea. Excluding some minor modifications to accommodate the new material selections, the array dimensions are the same as defined in the previous generation of Michigan cochlear probe [2].

\subsubsection{Width and Length}

The cat array substrates are $8 \mathrm{~mm}$ long and taper from $800 \mu \mathrm{m}$ to $380 \mu \mathrm{m}$, base to apex (Fig. 2.3). The guinea pig arrays are just as long, but narrower, tapering from 500 $\mu \mathrm{m}$ to $200 \mu \mathrm{m}$, base to apex (Fig. 2.4). Both prototype arrays incorporated a $0.7 \mathrm{~mm}$ wide $5 \mathrm{~cm}$-long monolithic parylene cable. This cable can be rivet bonded to a PCB for connection to a current stimulator. A shorter cable design can be used for connection to a multiplexing/stimulation ASIC.

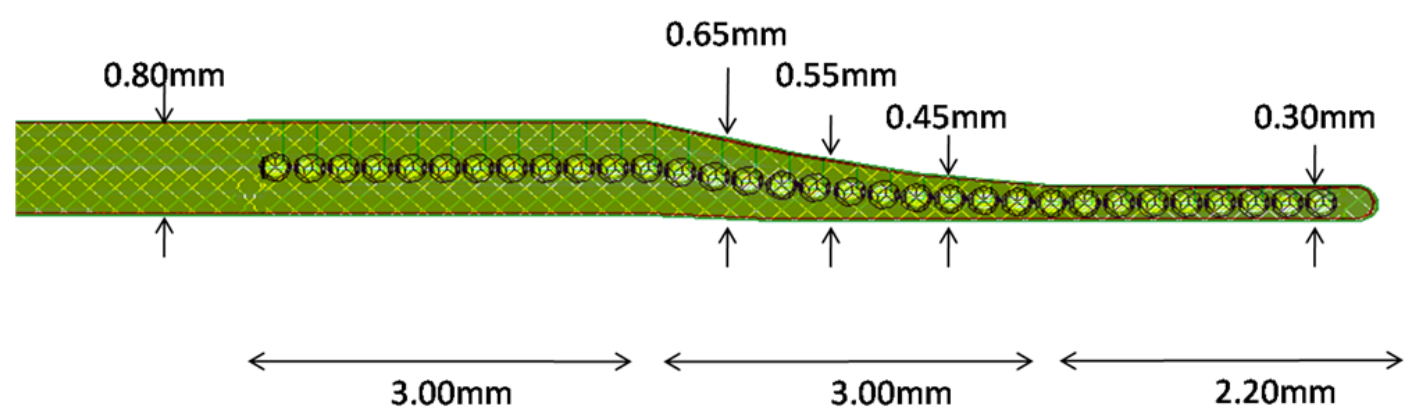

Fig. 2.3: Layout dimensions of parylene substrate cat arrays. 


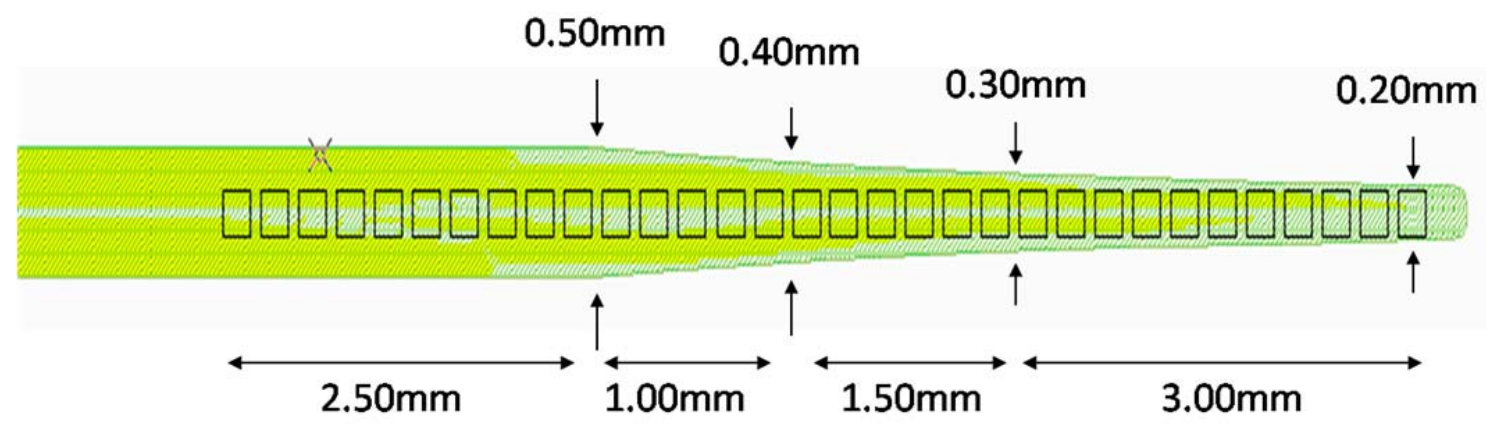

Fig. 2.4: Layout dimensions of parylene substrate guinea pig arrays.

The substrate dimensions for the guinea pig were chosen basedon cochlea crosssectional area data from the University of Washington. Data came from Voie and Spelman, who collected dimensional numbers on the GP cochlea by taking 2-D optical images of cochlea cross-sections and building it into a 3-D model [11]. In collaboration with Michigan Technological University (MTU), Ben Arcand of MTU translated both data sets into height and width dimensions for the GP cochlea under the assumption that the area cross-section is an ellipse. The minor axis represents the width of the cochlea and the major represents the height. Since the electrode array is vertically inserted into the cochlea, the height of the GP cochlear corresponds to the width of the array. The major and minor radii were calculated as a function of cochlear length $(x)$,

$$
\begin{aligned}
R_{\min } & =8.413\left(10^{-7}\right) x^{5}+1.161\left(10^{-5}\right) x^{4}-1.135\left(10^{-3}\right) x^{3}+1.903\left(10^{-1}\right) x^{2} \\
& -1.428\left(10^{-1}\right) x+0.5374 \\
R_{\text {maj }}= & -3.579\left(10^{-4}\right) x^{3}+1.240\left(10^{-2}\right) x^{2}-1.446\left(10^{-1}\right) x+0.7995 .
\end{aligned}
$$

Offsetting these radii calculations by $2 \mathrm{~mm}$, an estimate was made for the height and width of the GP cochlea (Fig. 2.5). As discussed in the next section, the electrode sites were chosen to have a diameter of $180 \mu \mathrm{m}$. To keep the width of the array slightly above the site diameter for fabrication ease, the length of the array was set to $8 \mathrm{~mm}, 6.6 \mathrm{~mm}$ shorter than the complete length of the GP cochlea. The width of the array design was decreased from the maximum allowable to prevent unnecessary damage to the cochlea during insertion. 


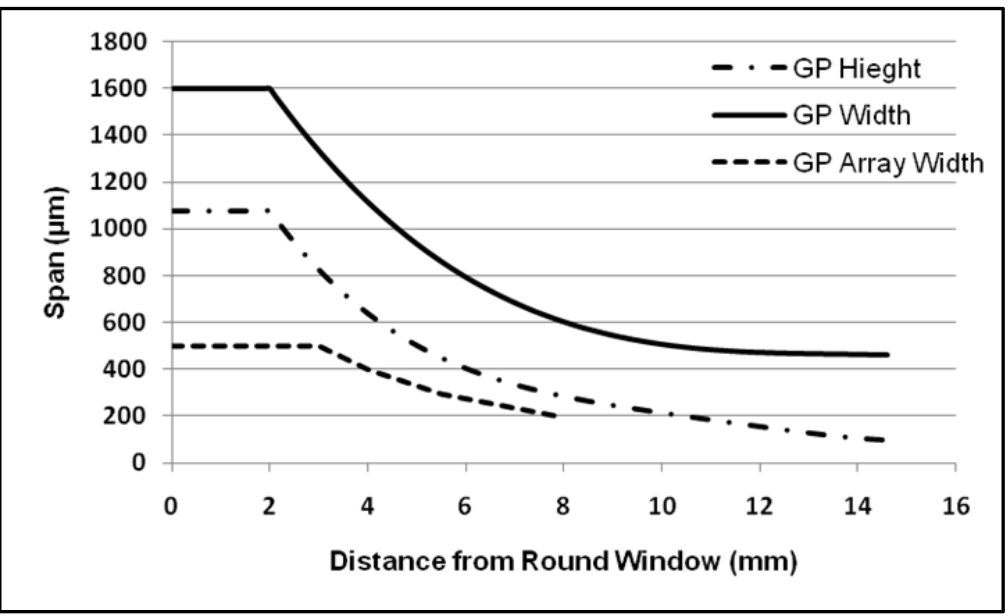

Fig. 2.5: Span of the GP height and width as extracted from Voie-Spelman dimensional data. The GP array width is based on these constraints.

While the GP cochlear is roughly four times smaller than the human cochlea, the crosssection of the cat cochlea is more comparable to that of the human and this makes it a better model to work with. The cross-sectional area of the cat is almost identical to the human at the round window and greater than the human within the first $1 \mathrm{~mm}$ (

Fig. 2.6, Fig. 2.7, Fig. 2.8). However, the cross-sectional area rapidly decreases over the next $6-8 \mathrm{~mm}$ to $25 \%$ to $30 \%$ of the human cochlea [12]. The cat prototype array design followed the general shape of the cat cochlea height, but included a reduction in size for insertion ease (Fig. 2.9).

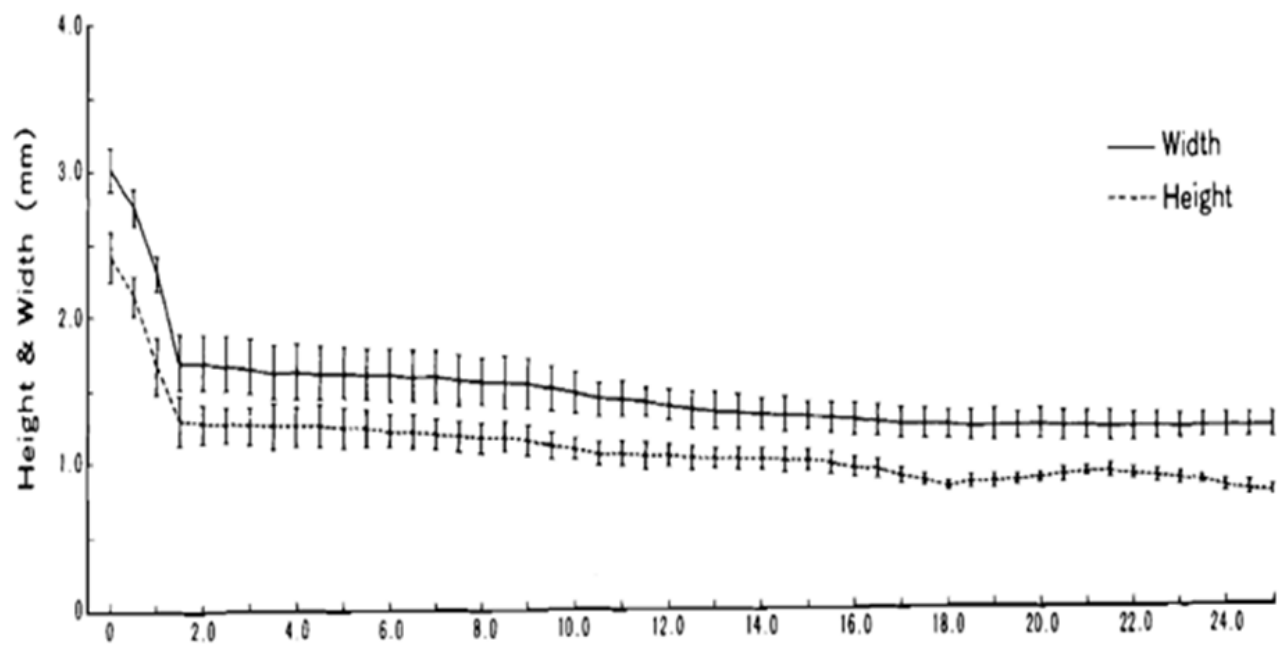

Fig. 2.6: Height and width of the human cochlea [12]. 


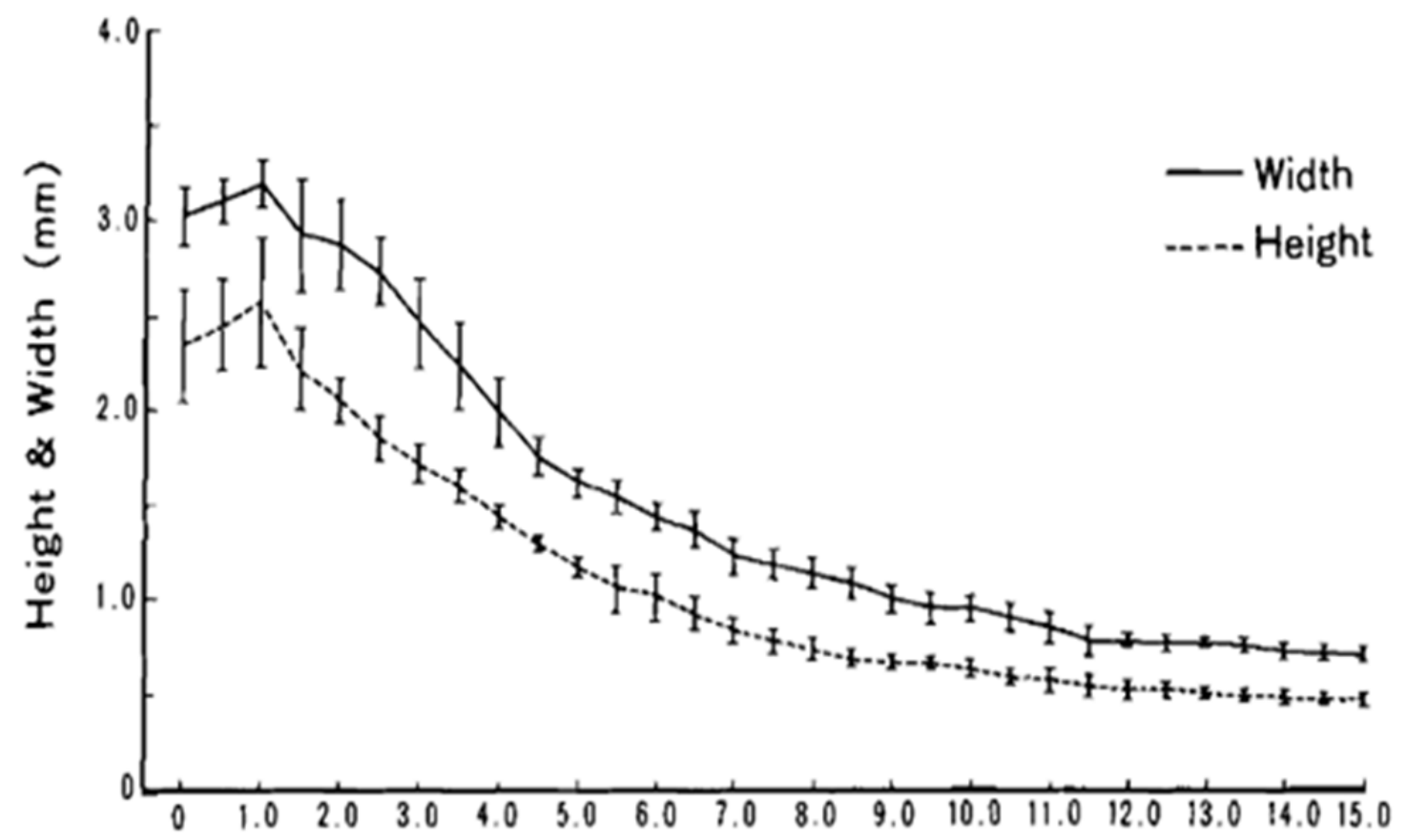

Fig. 2.7: Height and width of the cat cochlea [12].

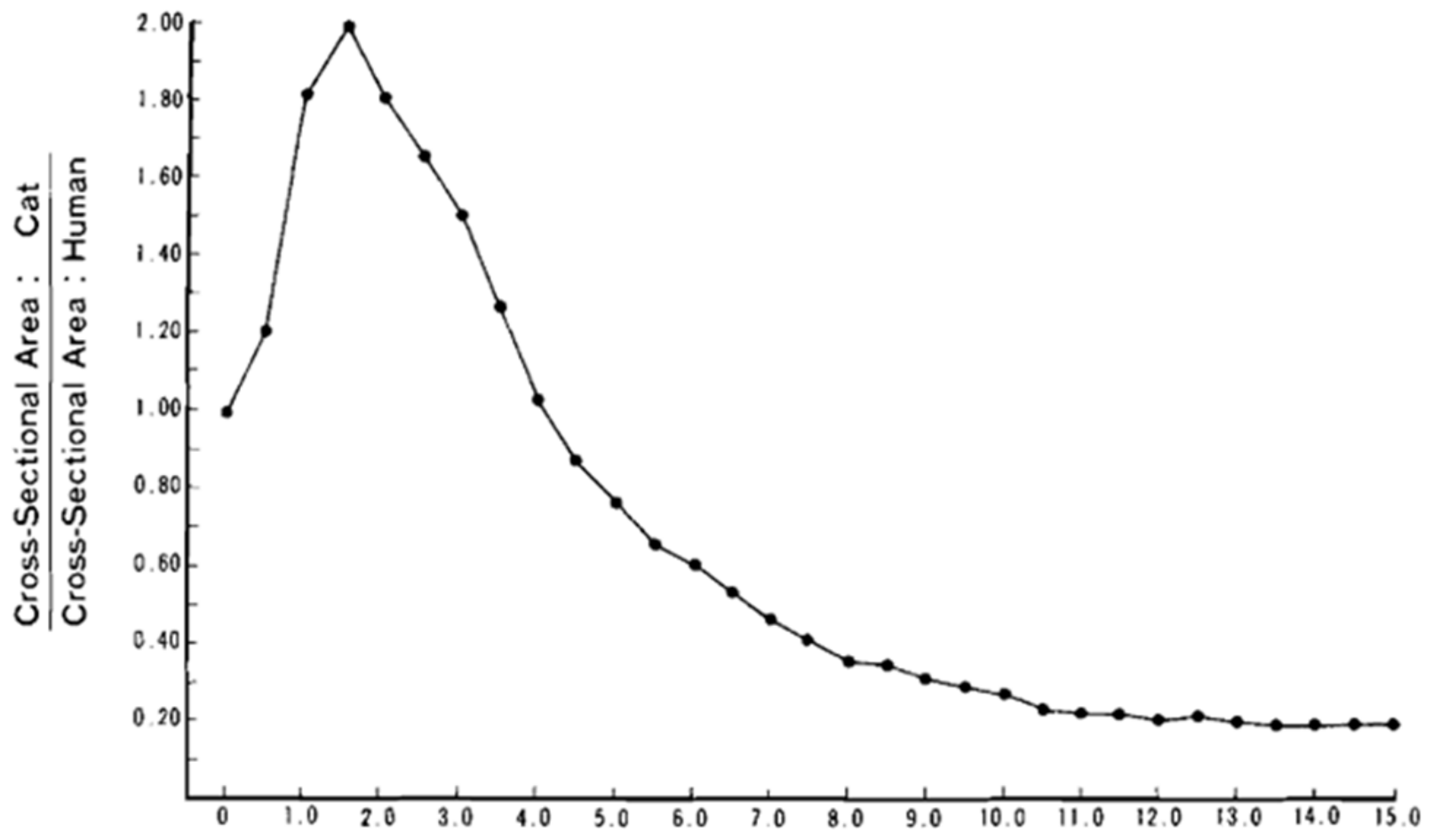

Fig. 2.8: Cross-sectional area of the cat cochlear relative to the human cochlea [12]. 


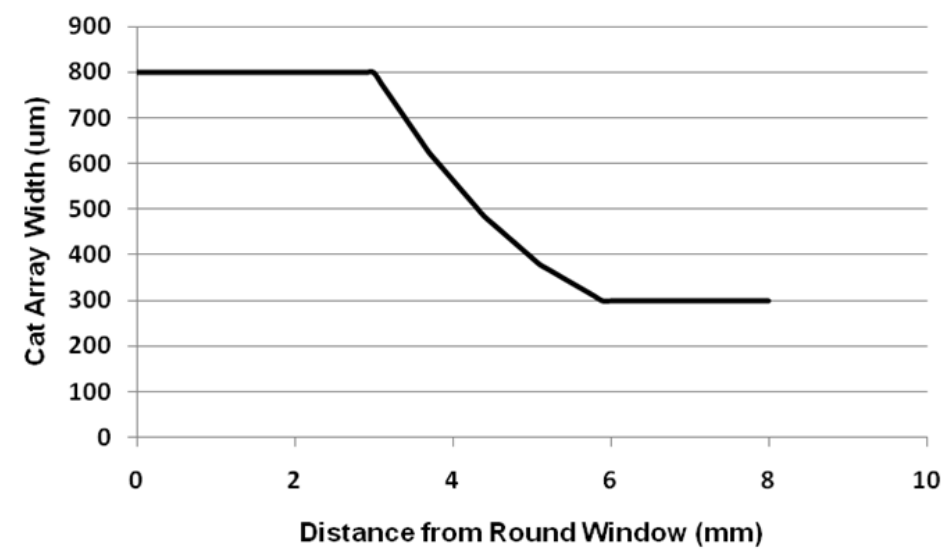

Fig. 2.9: Width of the cat array as a function of distance.

\subsubsection{Site Diameter}

When it came to choosing an appropriate site size, charge density and back voltage were considered most heavily since both determine the safety limits of charge delivery [2]. If either is too high, they produce irreversible Faradic reactions which lead to electrolysis and toxic products. The electrode array sites were designed to operate safely at current amplitudes as high as $500 \mu \mathrm{A}$ over pulse widths that ranged from 25 $200 \mu$ s (typical commercial pulse widths). Considering purely the charge density, it can be seen that sites smaller than $100 \mu \mathrm{m}$ in diameter are not suitable, given that the charge density limit for an $\mathrm{IrO}$ site is $1000 \mu \mathrm{C} / \mathrm{cm}^{2}$ [13].

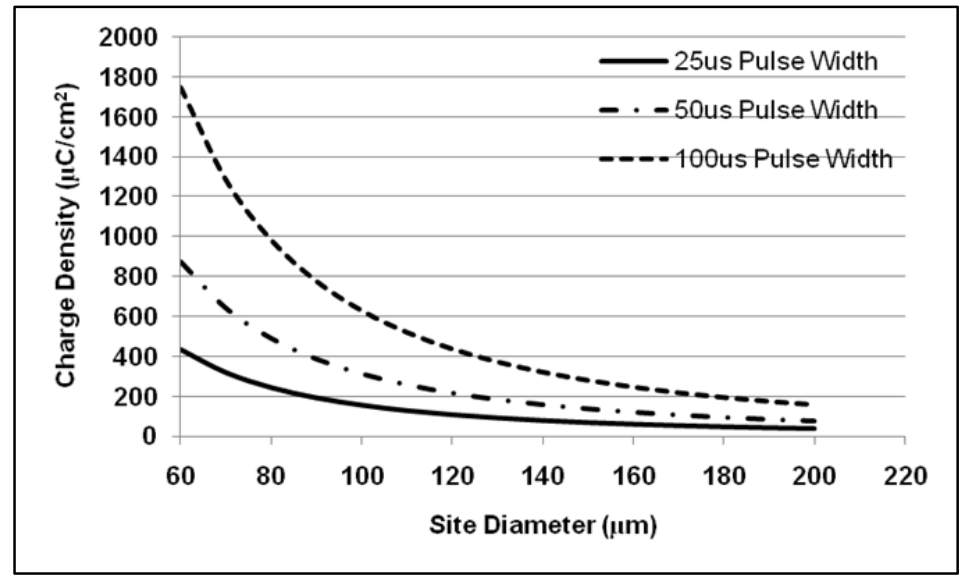

Fig. 2.10: Charge density as a function of electrode site diameter 
The back voltage (compliance voltage) of the electrode site is the potential between the current source output and the sink (reference electrode) in response to current stimulation. It is dependent on the charge transfer resistance $\left(\mathrm{R}_{\mathrm{ct}}\right)$, double layer capacitance $\left(\mathrm{C}_{\mathrm{dl}}\right)$, conduction path and spreading resistance of current flow through tissue $\left(R_{\mathrm{s}}\right)$. These relationship between these factors is depicted in the Randles model [ref] of electrode model (Fig. 2.11).

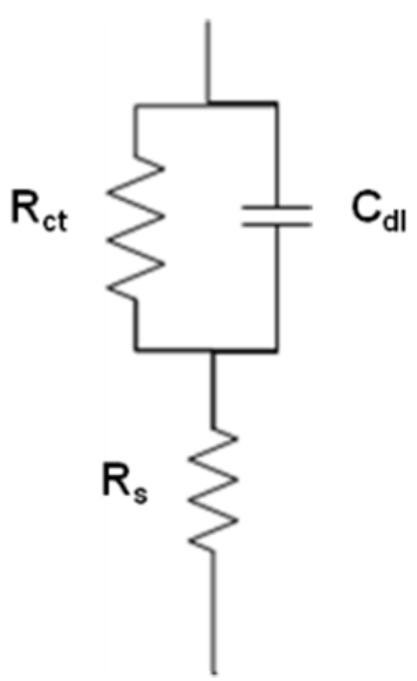

Fig. 2.11: The Randles electrode model depicting the charge transfer resistance, double layer capacitance, and spreading resistance.

With sufficient activation and surface roughness, the site impedance for the 180 $\mu \mathrm{m}$-diameter can be well under $2 \mathrm{k} \Omega$. This impedance contributes an estimated $1 \mathrm{~V}$ to the back voltage for a $500 \mu \mathrm{A}$ stimulus pulse. The other major contributor to the back voltage is the spreading resistance. In the original design of the sites, Bhatti used Kim's model for access (spreading) resistance [14]. This model is for hemispherical electrodes and assumes monopolar stimulation (ground much larger and farther than stimulating site). Kim defines the spreading resistance as

$$
R_{S} \approx 45 * \rho * \frac{\sin \theta}{\theta} * \frac{1}{\pi * r},
$$

where $\rho$ is the resistivity of the electrolyte ( $100 \Omega$-cm for perilymph), $\theta$ is the dispersion angle $\left(\theta=70^{\circ}\right)$, and $r$ is the electrode site radius. Using this equation, it can be seen that a back voltage $\leq 2 \mathrm{~V}$ can be achieved for electrode site diameters under $200 \mu \mathrm{m}$ (Fig. 2.12, Fig. 2.13). 


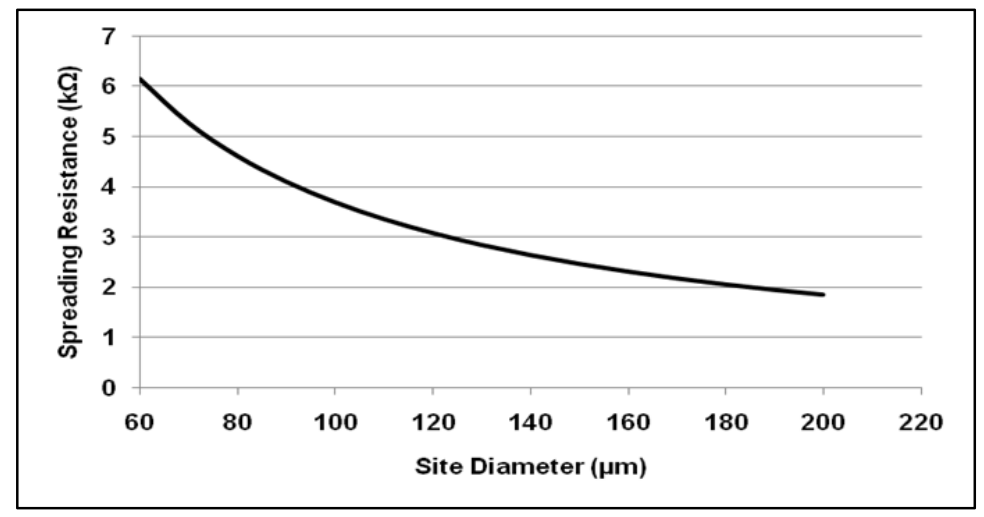

Fig. 2.12: Simulated spreading resistance as a function of site diameter.

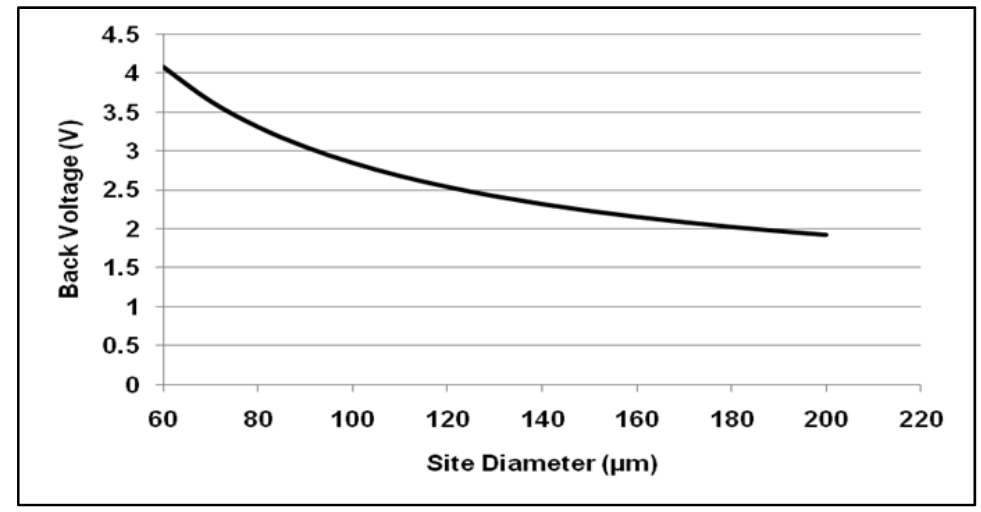

Fig. 2.13: Simulated back voltage in response to a $500 \mu \mathrm{A}$ stimulus pulse for $\mathrm{IrO}$ sites, versus diameter. Back-voltage includes contributions from spreading resistance and site-impedance.

In compliance with the electrical constraints for safe charge delivery, a site diameter of $180 \mu \mathrm{m}$ was chosen for the array design. This diameter minimizes charge density ( less than $200 \mu \mathrm{C} / \mathrm{cm}^{2}$ ) and back voltage while still allowing for 128 sites on a $35 \mathrm{~mm}$ length array, given a center-to-center site spacing of $250 \mu \mathrm{m}$.

\subsection{Fabrication Considerations}

In the course of fabricating the parylene arrays, several process issues had to be addressed. The most critical of these included choosing an appropriate sacrificial layer and release agent for the arrays, ensuring good site adhesion to the inert substrate, ensuring proper adhesion of parylene to itself for the stacking of layers, and defining 
small line pitches of $10 \mu \mathrm{m}$ to demonstrate the feasibility of a 128 -site array. The following sections discuss how these areas were addressed.

\subsubsection{Sacrificial layer}

The inert parylene layers need to be securely fixed to the carrier during processing, but easily released at the end of fabrication. One way to achieve this is to use a sacrificial layer in-between the parylene and the silicon substrate. The parylene could be fixed to this sacrificial layer with A-174 adhesion promoter and released at the end of processing by etching. Several sacrificial layers were considered and tested. These layers included photoresist, low temperature PECVD oxide, and aluminum (Al).

Photoresist has been used by others as a release layer for parylene_[15]. It is the most convenient candidate since it is easy to coat and can be readily removed with acetone in less than an hour. To build a photoresist sacrificial layer, Shipley photoresist 1813 was spin-coated on the silicon carrier to a thickness $1 \mu \mathrm{m}$ and the baked for 1 hour at $110^{\circ} \mathrm{C}$ to remove solvents. The photoresist sacrificial layer stood up against most processing steps, even those that included quick rinses in acetone, but it did not survive the liftoff process, which required long soaks in acetone. During liftoff, acetone was absorbed by the parylene and attacked the photoresist sacrificial layer. Although it did not immediately remove the layer, it did cause the resist to out-gas during subsequent pre-exposure bakes, and this eventually resulted in the parylene delaminating from the carrier prematurely.

PECVD oxide was also explored as a sacrificial layer. Initially, to release the oxide layer, a solution of 10 parts dionized (DI) water to one part hydrofluoric acid (HF) was used. With this process, the arrays released in under 2 hrs. However, this solution attacked the Ti adhesion layer of the Ti-Ir electrode sites (Fig. 2.14). It was observed that the HF solution went through the parylene top layer. With an etch rate of $1100 \AA / \mathrm{min}$ in 10:1 HF [16], the Ti adhesion layer was easily attacked at its interface with the parylene surface. Similar observations of HF being absorbed by parylene have been reported by others [17]. With an etch rate for Ti of less than $2 \AA / \min$ [16], Pad Etchant Type 4 was tested as a substitute for HF. Although the rate was slower with the Pad Etch, it also attacked the adhesion between the Ti-Ir sites and the parylene. When the sites were 
comprised of Ti alone and no Ir, they did not delaminate, even after an overnight soak in pad etchant. This suggests that the compressive force of the Ir over the Ti is what makes it susceptible to delamination once softened by the wet etchants.

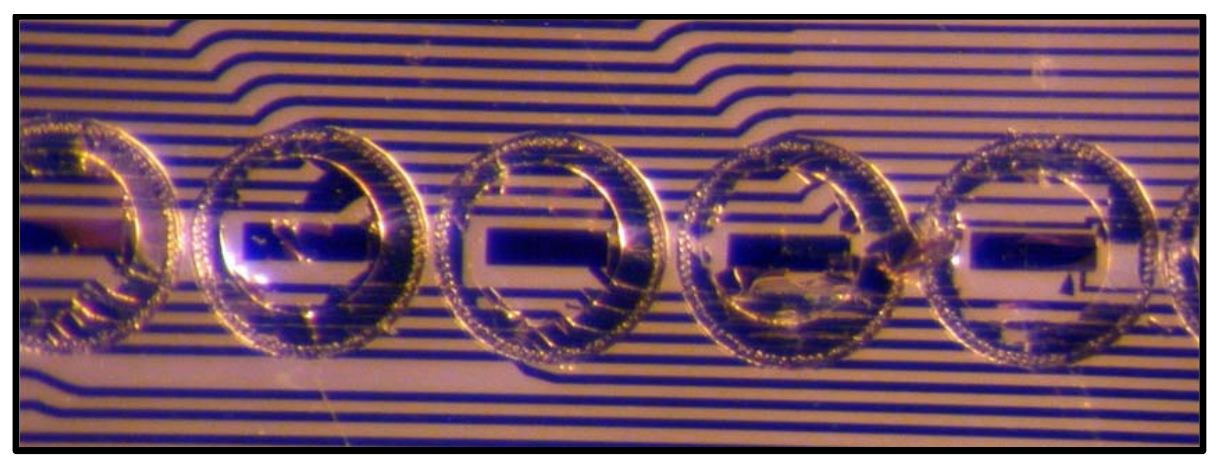

Fig. 2.14: Ti-Ir sites fractured and delaminated from parylene substrate, post release.

As an alternative to the PECVD oxide, a $1000 \AA$-thick Al sacrificial layer was considered. This sacrificial layer was released using Al etchant type A. The etchant was heated to $90^{\circ} \mathrm{C}$ in a $200 \mathrm{~mL}$ beaker and a stirrer was used to agitate the solution. The arrays were inserted in a Teflon wafer carrier upside down, and the carrier was placed in the beaker with the top-side of the wafer facing the stirrer. The etch rate of $\mathrm{Al}$ alone in the hot solution was recorded to be $100 \AA /$ s. However, the time to release the arrays was greater than $20 \mathrm{hrs}$. After $20 \mathrm{hrs}$ the arrays did not fully release, and there remained residual Al under the devices (Fig. 2.15). The increased etch rate is thought to result from the inability of by-products produced by the etching process to be released from under the parylene arrays.

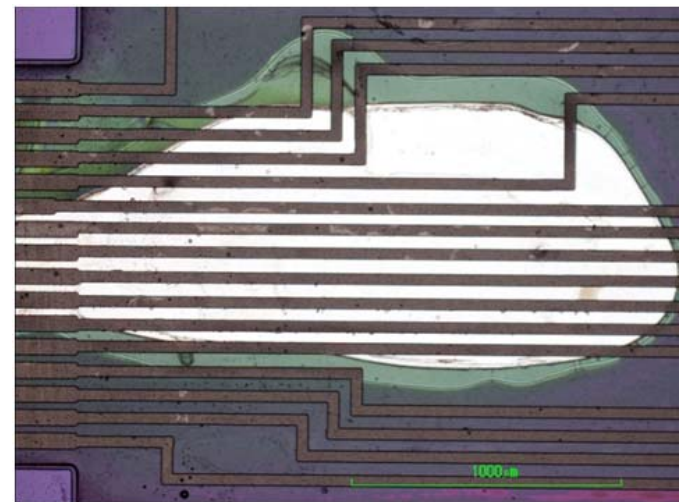

Fig. 2.15: Residual aluminum sacrificial layer under a cochlear array after more than $20 \mathrm{hrs}$ of soaking in agitated $\mathrm{Al}$ etchant type $\mathrm{A}$ at $90^{\circ} \mathrm{C}$. 
Since an appropriate sacrificial layer could not readily be found, array processing was attempted without a sacrificial layer at all. Instead, 50 $\mu 1$ of A-174 adhesion promoter was used in the SCS PDS2035 parylene coater. The normal amount of adhesion promoter to be applied is $10 \mathrm{~mL}$, with this reduced amount, the parylene arrays could be released in $2 \%$ potassium hydroxide $(\mathrm{KOH})$ solution at room temperature within 8 hours. In such a diluted solution of $\mathrm{KOH}$, the occurrence of delaminated Ti-Ir sites was much less than for any of the previously-used wet etchants. Partial delamination of the sites did still occur, though at release times greater than 5 hours. To reduce the amount of time needed to release the arrays in $2 \% \mathrm{KOH}$, the adhesion promoter was eliminated all together. As expected, lack of any adhesion promoter had initially led to severe parylene bubbling during processing (Fig. 2.16). The bubbling occurred as absorbed solvents delaminated the parylene from the silicon carrier. When the wafers were placed on the hotplate for lithographical processing steps, the heat expanded the trapped solvents and further exacerbated the adhesion issues, in some cases tearing the parylene altogether ( Fig. 2.17). This significantly reduced device yield.

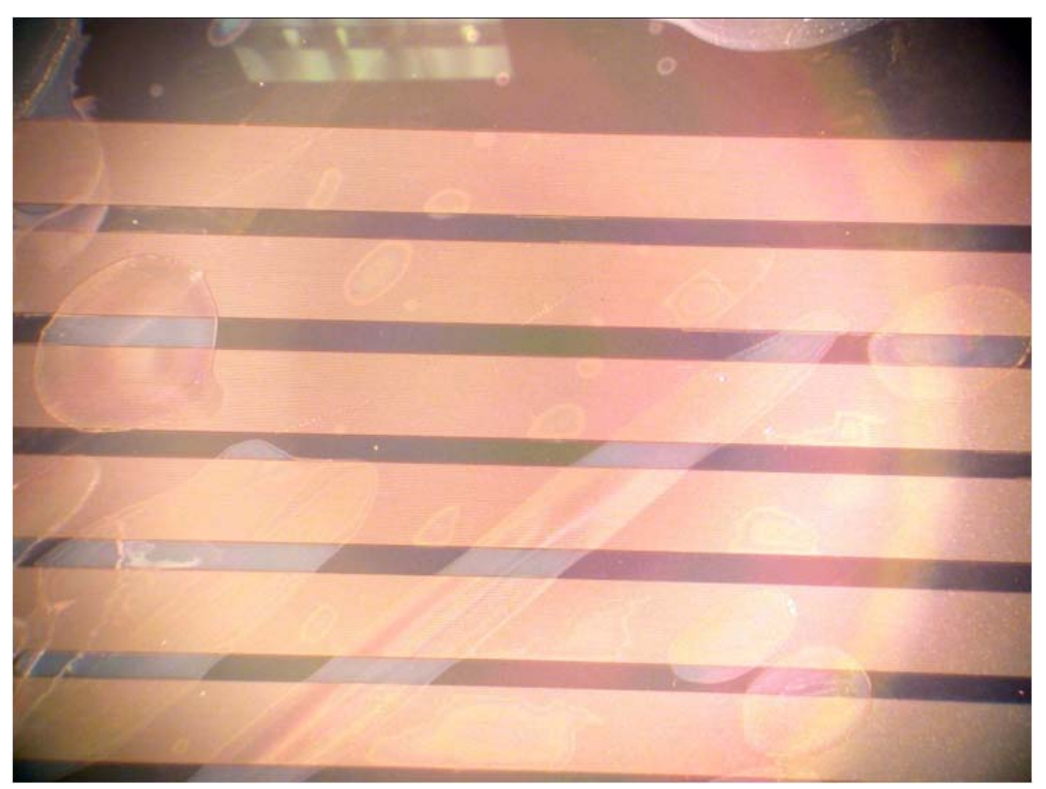

Fig. 2.16: Delamination of parylene from silicon surface due to lack of an adhesion promoter. 


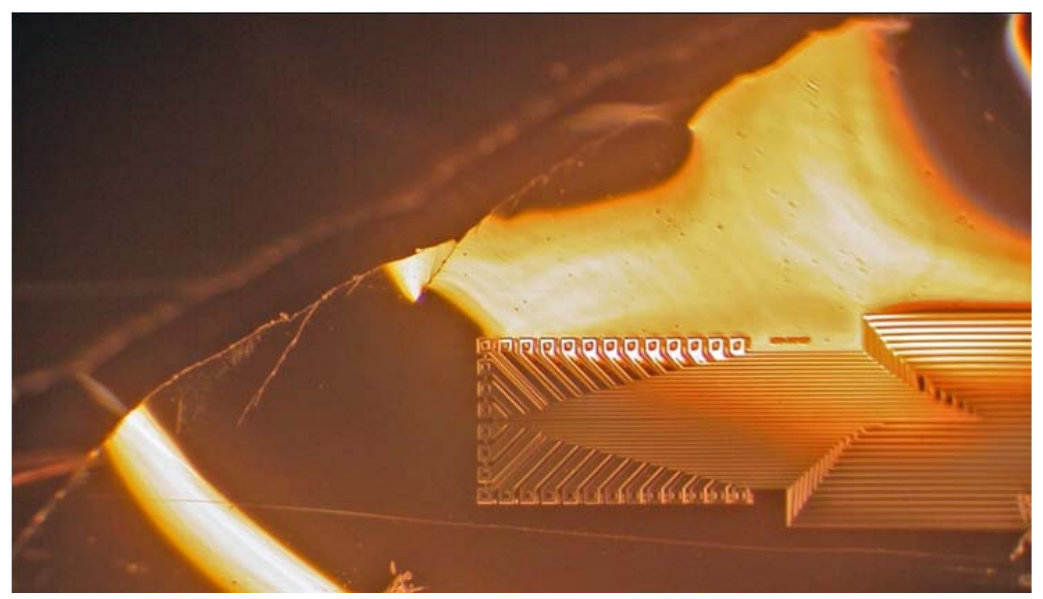

Fig. 2.17: Burst parylene substrate due to out-gassing of solvents.

Through optical observation of the parylene-to silicon-adhesion after each subsequent process step, it was noted that certain developers and photoresists increased delamination more than others. Of the photoresists, AZ 9260 photoresist, Microposit 1813, and Microposit 1827 resulted in the highest degree of delimination. AZ 9260 resist is stickier than the rest and during contact alignment it would forcibly pull the parylene away from the carrier. Developing the resist in a solution of one part AZ $400 \mathrm{~K}$ to three parts DI water further increased delamination. The effect of Microposit 1827 and 1813 on delimation was not as severe as 9260 , but a small degree of delimination did occur with their use. In contrast, SPR 220-3 showed no visible effect on the parylene-to-silicon adhesion in either the contact alignment step or the developer (Microposit MF 319 or AZ MIF 300). As such, all array lithography steps up to the final oxygen plasma etch are done with SPR 220-3( Fig. 2.3e). The resist used for this final etch is AZ 9260, since none of the other resists provide a thick enough mask for the etching of $11 \mu \mathrm{m}$ of parylene in an RIE oxygen plasma.

\subsubsection{Ti-Ir Adhesion}

Apart from any peeling that can occur on a Ti-Ir site as a result of wet etchant releases, delamination can occur pre-release or be exacerbated during release by insufficient adhesion between the site and the parylene substrate at the time of deposition. The degree to which the Ti-Ir sites adhere to the parylene is most dependent on the stress in the layer. This stress can increase or decrease as a result of the sputter deposition base 
pressure, deposition pressure and deposition power. Using the Denton explorer sputter tool, Ti-Ir $(200 \AA / 1500 \AA)$ was deposited at two separate combinations of deposition power and pressure, $500 \mathrm{~W} / 3 \mathrm{mT}$ and $300 \mathrm{~W} / 7 \mathrm{mT}$. The stress values were acquired using the Flexus thin-film stress measurement system. This system calculates stress by measuring the difference in wafer curvature that arises from Ti-Ir deposition. For the $500 \mathrm{~W} / 7 \mathrm{mT}$ depositions, the Ti-Ir stress varied by less than $100 \mathrm{MPa}$ at base pressures ranging from $10 \mathrm{nTorr}$ down to $2 \mathrm{nTorr}$. The stress at $10 \mathrm{nTorr}$ was equal to $2.03 \mathrm{GPa}$ compressive, and the stress at 2 nTorr was equal to $1.94 \mathrm{GPa}$. This high compressive stress sometimes resulted in the electrode sites delaminating from the parylene substrate (Fig. 2.18).

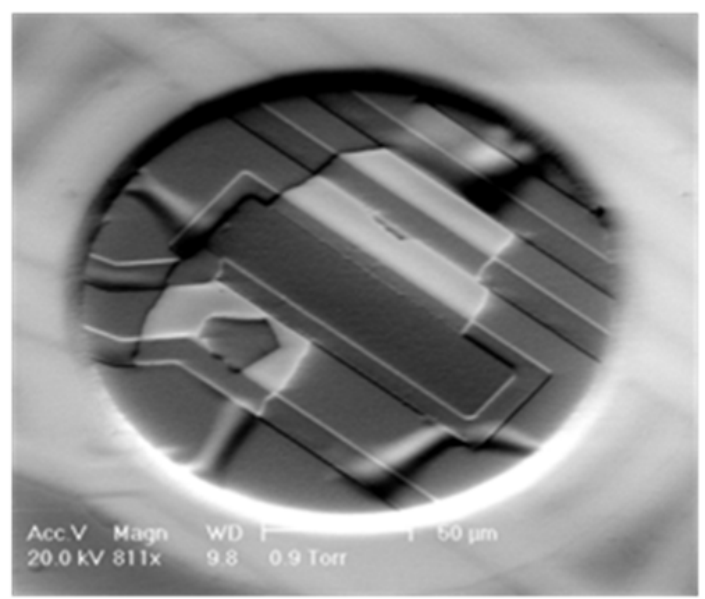

Fig. 2.18: Buckling and fracturing of a Ti-Ir site under high compressive stresses greater than $2 \mathrm{GPa}$ compressive.

For $300 \mathrm{~W} / 7 \mathrm{mT}$ depositions, the relationship between stress and base pressure was much greater. At $10 \mathrm{nTorr}$, the stress was $312 \mathrm{MPa}$ compressive, and at $5 \mathrm{nTorr}$ it was $144 \mathrm{MPa}$ tensile. It should be noted that the values of stress changed quite a bit from run to run and depended on the working condition of the sputter tool. However, for all runs the stress was more tensile with lower base pressures and lower deposition power. Increases in deposition pressure also trended toward more tensile Ti-Ir layers, but with the variations in tool performance, the data collected was not entirely conclusive towards this point. Optimum run parameters included a base pressure of 2 nTorr or less, a sputter 
power of $300 \mathrm{~W}$, and a sputter pressure of $8.5 \mathrm{mT}$ Torr. With these parameters, no delamination of the sites were seen pre- or post-release (Fig. 2.19).

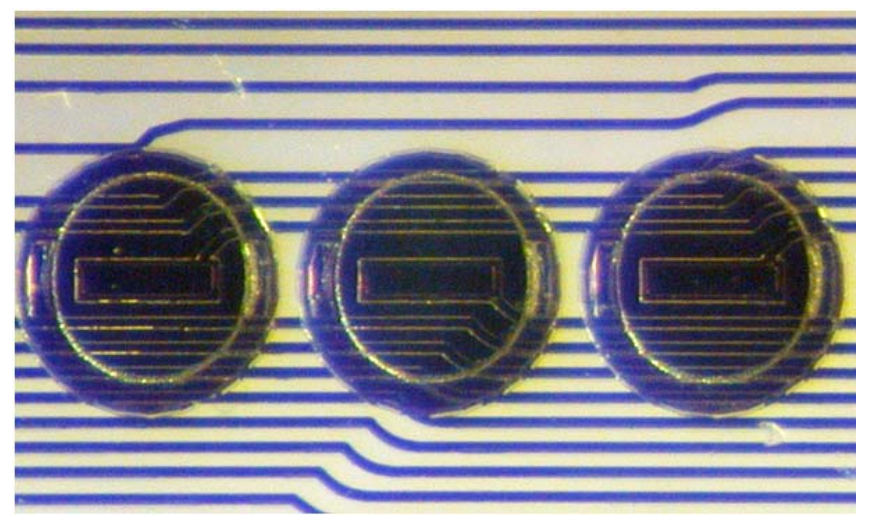

Fig. 2.19: Securely adhered Ti-Ir sites on parylene substrate arrays, post-release.

\subsubsection{Interconnect Pitch}

Line pitches of $20 \mu \mathrm{m}$ were sufficient to produce 32-site cat arrays, giving a width tapering of $800 \mu \mathrm{m}$ to $380 \mu \mathrm{m}$, base to apex (Fig. 2.3). However, for guinea pig arrays, the width tapered from $500 \mu \mathrm{m}$ to $200 \mu \mathrm{m}$, base to apex (Fig. 2.4), and it became necessary to shrink the pitch in half (down to $10 \mu \mathrm{m}$ ). Typically, to create $5 \mu \mathrm{m}$ traces, spaced $5 \mu \mathrm{m}$ apart, techniques such as ultrasonic agitation or hot 1112A baths are used. However, on parylene surfaces this type of agitation resulted in the partial removal of some traces (Fig. 2.20). Without agitation, the liftoff process was often incomplete when using one coat of photoresist (AZ 9260 photoresist, Microposit 1813, Microposit 1827, or SPR 220-3) and standard contact alignment exposure. Incomplete lifting off of the $\mathrm{Cr}-\mathrm{Au}-\mathrm{Cr}$ interconnect resulted in metal in-between traces clinging to the parylene substrate or flipping over the trace lines (Fig. 2.21). 


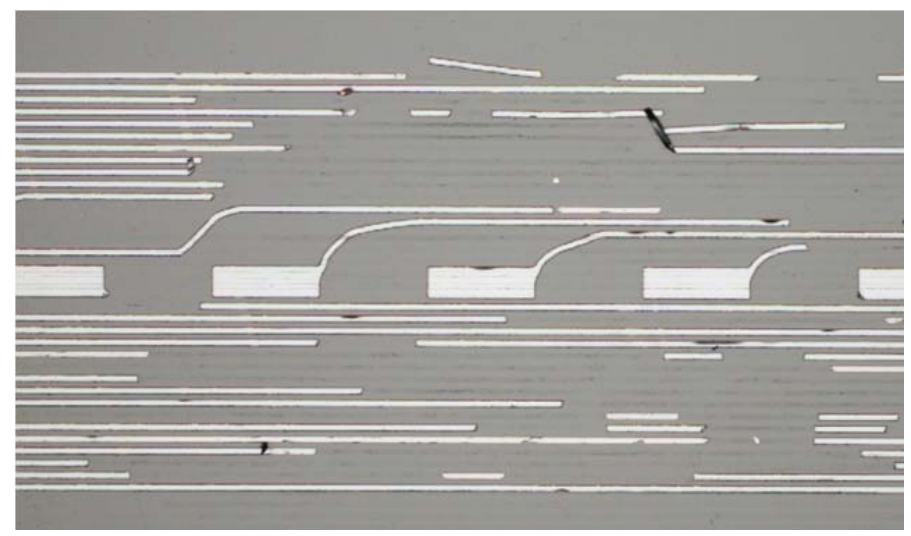

Fig. 2.20: Delaminated interconnect resulting from ultrasonic agitation of wafers in acetone.

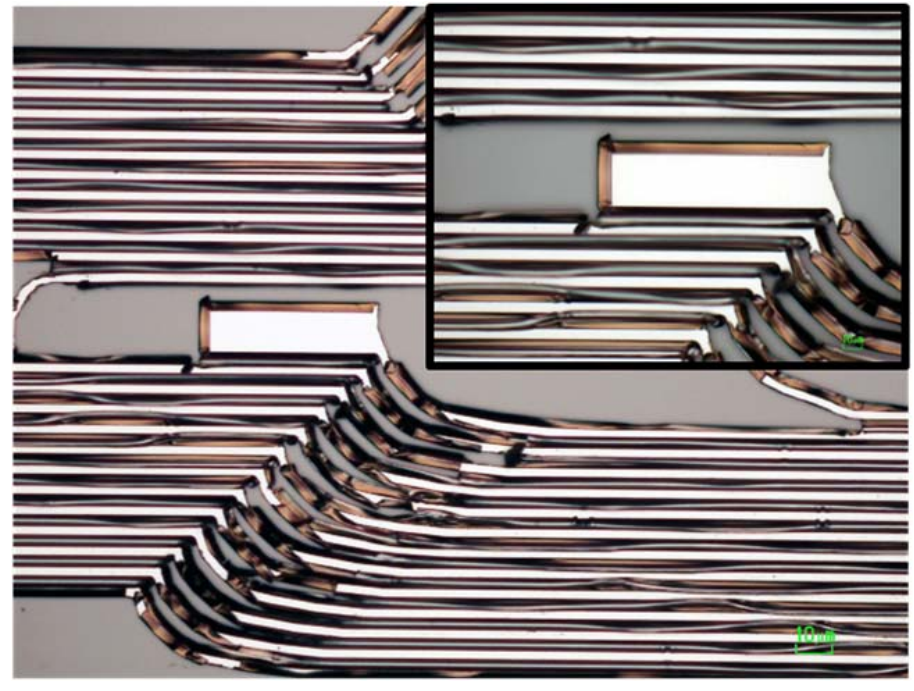

Fig. 2.21: Single layer, standard lithography producing incomplete liftoff of $\mathrm{Cr}-$ $\mathrm{Au}-\mathrm{Cr}$ on a parylene substrate.

Both effects were a result of improperly-angled side-wall features in the resist following development. To deposit the $\mathrm{Cr}-\mathrm{Au}-\mathrm{Cr}$ layers, an Enerjet E-beam evaporator was used. With this method, deposition is highly non-conformal and line-of-sight. This leads to the bottoms of trenches getting coated to a higher degree than the side-walls. Depending on side-wall angle, the non-conformal nature of the deposition can be increased or lessened. Angles of $90^{\circ}$ have poor conformality, but resist does partially coat the side-walls (Fig. 2.22). As the angle shrinks from $90^{\circ}$ towards $0^{\circ}$, the coatings become increasing non-conformal (Fig. 2.23). 
(a)

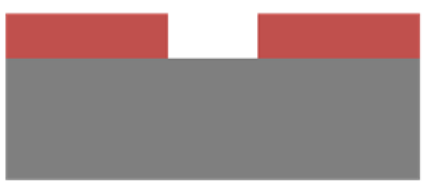

(b)

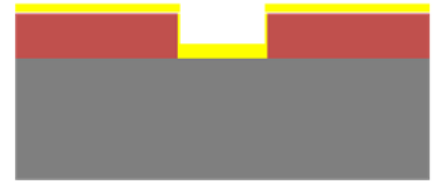

(c)

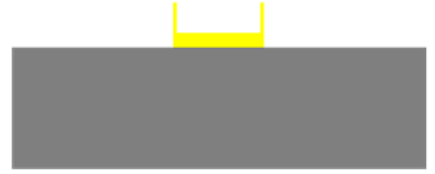

Fig. 2.22: Standard lithography producing a $90^{\circ}$ side-wall angle, (a) $90^{\circ}$ side-wall angle in resist after exposure and development, (b) metal deposition coating the bottom of a trench more than the side-walls, (c) poor liftoff, with metal on sidewalls clinging to trace lines.

(a)

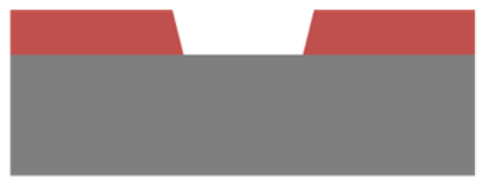

(b)

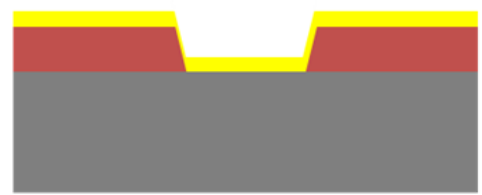

(c)

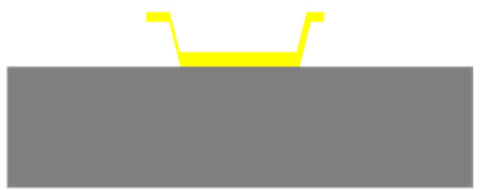

Fig. 2.23: Standard lithography producing less than $90^{\circ}$ side-wall angles. (a) less than $90^{\circ}$ side-wall angle in resist after exposure and development, (b) metal deposition coating the bottom of a trench more than the side-walls, (c) poor liftoff, with metal on the side-walls and top surface of the resist clinging to the trace lines.

For liftoff purposes, the less conformal the coating the better. Surface "hardening" and double-layer resist techniques were explored to modify the side-wall angle for improved liftoff. The most successful approaches included using double-layer photoresist lithography (

Fig. 2.24) and "hardening" the surface of the resist with either chlorobenzene or TMAH (Fig. 2.25).

(a)

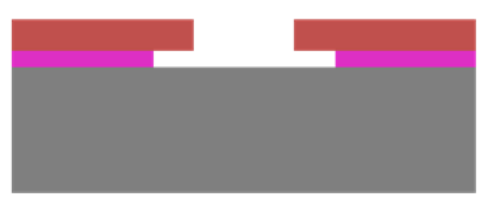

(b)

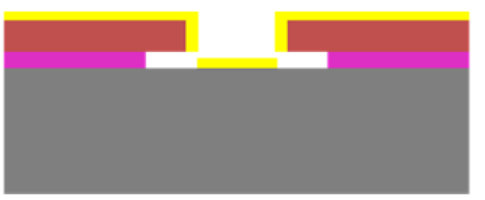

(c)

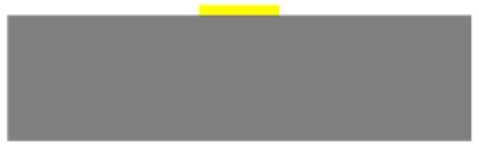

Fig. 2.24: Slowly developing the top resist with respect to the bottom produces recessed side-walls. (a) Difference in developing rate between top and bottom resist results in recessed side-walls, (b) No contact of metal on the remaining sidewall with traces at the bottom of the trench, (c) unwanted metal lifts off easily with resist, leaving clean trace lines behind. 
(a)

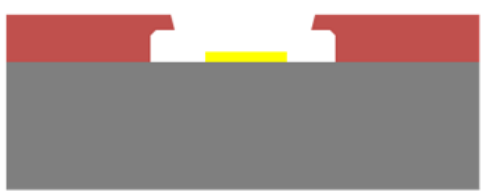

(b)

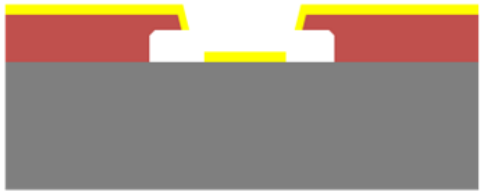

(c)

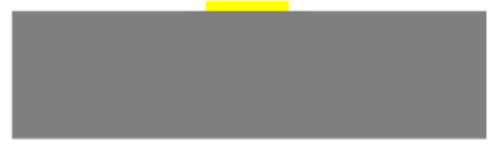

Fig. 2.25: Surface "hardening" of resist to produce recessed side-walls. (a) Reduced solubility of the top surface producing recesses as the bulk etches faster, (b) No contact of metal on the remain side-wall with traces at the bottom of the trench, (c) unwanted metal lifts off easily with resist, leaving clean trace lines behind.

Of these techniques, the only one to work consistently over multiple runs was the TMAH surface hardening technique. This method was first introduced by Redd, et al., on silicon surfaces and was later used by Seymour on parylene surfaces [18,19]. A variation of the technique was used in the array process. It consisted of spin-coating HMDS at $4 \mathrm{~K}$ rpm on the parylene surface followed by pin-coating SPR $220-3$ at $5 \mathrm{~K} \mathrm{rpm}$ for $30 \mathrm{~s}$. Next, the freshly spun wafers are spray coated with AZ MIF 300 developer for 20s and then subjected to a pre-exposure bake of $90^{\circ} \mathrm{C}$ for 2 minutes. The TMAH of the developer is absorbed into the top surface of the unbaked resist, and during the pre-exposure bake it acts as a catalyst for a chemical reaction which greatly reduces its solubility in future development steps. Following the pre-exposure bake, the resist is exposed for $4 \mathrm{~s}$ with the interconnect mask and baked again for another 2 minutes at $90^{\circ} \mathrm{C}$. The interconnect pattern is then spray-coated with MF 319 for a total of $45 \mathrm{~s}$. With this technique, the 10 $\mu \mathrm{m}$ line pitches were achieved after soaking the wafers in acetone for less than $5 \mathrm{hrs}$ (Fig. 2.26).

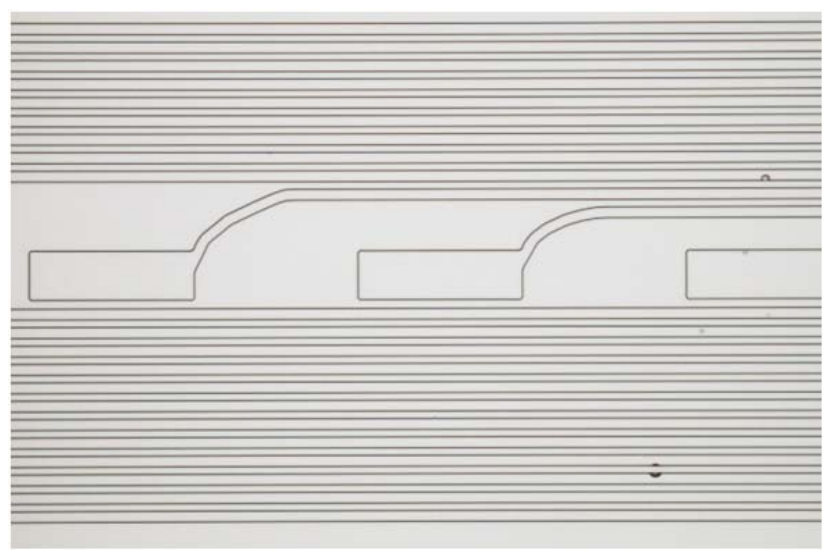

Fig. 2.26: TMAH liftoff of $\mathrm{Cr}-\mathrm{Au}-\mathrm{Cr}$ interconnect with $10 \mu \mathrm{m}$ pitch lines. 


\subsubsection{Parylene Adhesion}

When it comes to processing with parylene $\mathrm{C}$, the same material properties that make it an excellent cochlear array substrate also introduce complexities into the fabrication process. Parylene-C is an inert material so the monomer does not react with the surfaces it comes into contact with and so adhesion to most substrates is quite poor, including adhesion to Parylene-C itself. For the cochlear array design, it is important that the parylene layers sandwiching the electrical interconnect do not become separated in use and allow body fluid to cause shorting between the leads and sites.

To improve the adhesion of parylene-C to various substrates, a variety of techniques are used, with plasma treatment, silane promoters, and mechanical anchoring being the most common. Plasma treatment of parylene surfaces has been done in the past to roughen and clean the surface before parylene deposition [20,21,22]. It has achieved limited success, but is best when used in conjunction with the methods of mechanical anchoring, silane adhesion promotion, and thermal bonding.

Silane promoters can be used on surfaces that contain a reactive hydroxyl group, which is the case for most metals and silicon surfaces. A-174 adhesion promoter is used in this way. The silane molecule is functional at both ends with hydroxyl groups. One end bonds to the substrate and the free end is available to react with the gaseous paraxylene monomers as they make contact with the surface. For surfaces that do not contain a hydroxyl group readily available for reaction, which is the case with parylene substrates, silanes offer no benefit and in these cases mechanical anchors, thermal bonding, plasma treatment or a combination of each is used to promote adhesion. In fact, the use of A-174 on parylene substrates actually reduces adhesion to subsequent parylene layers. This is because the silane molecules form a thin detached layer between the two parylene layers. During deposition, the hydroxyl group at one end of the silane molecule will attached to the newly deposited parylene, but the other end will just merely lay upon the non-reactive surface of the previously-deposited non-reactive parylene substrate.

Mechanical anchors have been used to fix parylene to silicon substrates [17], and similar techniques could be used to anchor parylene to itself. In a study done by Rodger, et al., an array of anchors and a continuous trench were investigated to improve the adhesion of parylene to silicon. Both anchor types prevented chemicals such as HF from 
laterally seeping into the parylene-silicon interface, but only near the anchored regions. As such, the continuous trench made a better barrier against lateral attack since it did not contain unprotected gaps. However, in all anchor cases, the chemicals did get to the interface by vertically diffusing through the top of the parylene surface. Once they diffused in, they were easily able to build up between the poorly-adhering surfaces. This vertical attack resulted in delamination of the non-anchored regions and collection of gases and fluids between them [17]. This study suggests that merely anchoring parylene to itself in select regions is not sufficient to prevent delamination and the buildup of fluid in non-anchored regions. In the same study, annealing of the parylene at $180^{\circ} \mathrm{C}$ showed marked improvement over just anchoring. It is likely that the heat caused bonding between the parylene and the silicon in all contact areas. Later studies from the same group further investigated this concept and other adhesion enhancement techniques [23]. It was found that annealing of the stacked layers at $200^{\circ} \mathrm{C}$ in a vacuum oven combined with anchoring prevented both lateral and vertical delamination under accelerated soak tests $\left(60^{\circ} \mathrm{C}, 80^{\circ} \mathrm{C}, 90^{\circ} \mathrm{C}\right)$ for a period of 30 days. All of the previously mentioned studies were done to investigate parylene adhesion to silicon, but some of the same effects can be seen for parylene adhesion to itself. Many have used thermal heating of stacked parylene layers to fuse multiple layers [24, 25, 26, and 19]. When parylene-C layers are heated to high temperatures between their glass transition temperature $\left(\sim 90^{\circ} \mathrm{C}\right)$ and its melting temperature $\left(290^{\circ} \mathrm{C}\right)$ and are placed in close contact to each other, their polymer chains move and become entangled [24]. Factors such as temperature, pressure, and time affect the degree of entanglement. Kim, et al., looked into the thermal bonding of two parylene-coated silicon wafers under vacuum and pressure. With bonding conditions of $230^{\circ} \mathrm{C}$, a vacuum of $0.153 \mathrm{mbar}$, and $800 \mathrm{~N}$ of force, they were able to bond parylene to a strength of 3.60MPa. Although Kim, et al., did not conduct accelerated saline soak tests, they did assess the effects of various processing chemicals on the bond. For various solvents, bases, and acids, they observed less than an $11 \%$ change in bond strength after no more than 1 hour of soaking. Bonds soaked in acetone and IPA for a week showed a $20 \%$ and $8 \%$ drop in bond strength, respectively. Similar parylene bonding techniques have been done using microwave heating of the parylene at atmosphere to $160^{\circ} \mathrm{C}$ for 30 minutes under a pressure of 1.5 MPa [26]. Bond strengths as high as $12.68 \mathrm{MPa}$ for shear 
stresses and 9.16 MPa for tensile stresses were achieved. In all bonding strength tests the primary failure mechanism observed was tearing of parylene itself versus interfacial delamination. The parylene typically tore at a distance above the bond interface. Although pressure improves its strength, bonding can occur at high temperatures without the use of it. Rodger, et al., heated the stacked parylene layers of their retinal prosthesis in a vacuum oven at 10 Torr and $200^{\circ} \mathrm{C}$ for two days. Accelerated soak tests reported sufficient durability of adhesion of more than 20 years at $37^{\circ} \mathrm{C}$ [25]. Seymour placed parylene-encased interdigitated electrodes in a $140^{\circ} \mathrm{C}$ oven for $3 \mathrm{hrs}$ and observed no appreciable decrease in impedance to solution after 60 days of soaking in $37^{\circ} \mathrm{C} 1 \mathrm{X}$ PBS solution. The cochlear arrays of this work were fabricated using vacuum oven heating. To promote adhesion, the released parylene arrays are heated in a nitrogen-purged vacuum oven for $12 \mathrm{hrs}$.

\subsection{Array Fabrication}

The non-backed cochlear arrays were fabricated using alternating depositions of parylene-C and metal thin-film layers. Fabrication begins with the vapor deposition of a $5 \mu \mathrm{m}$-thick layer of parylene on a silicon carrier wafer (Fig. 2.27a).

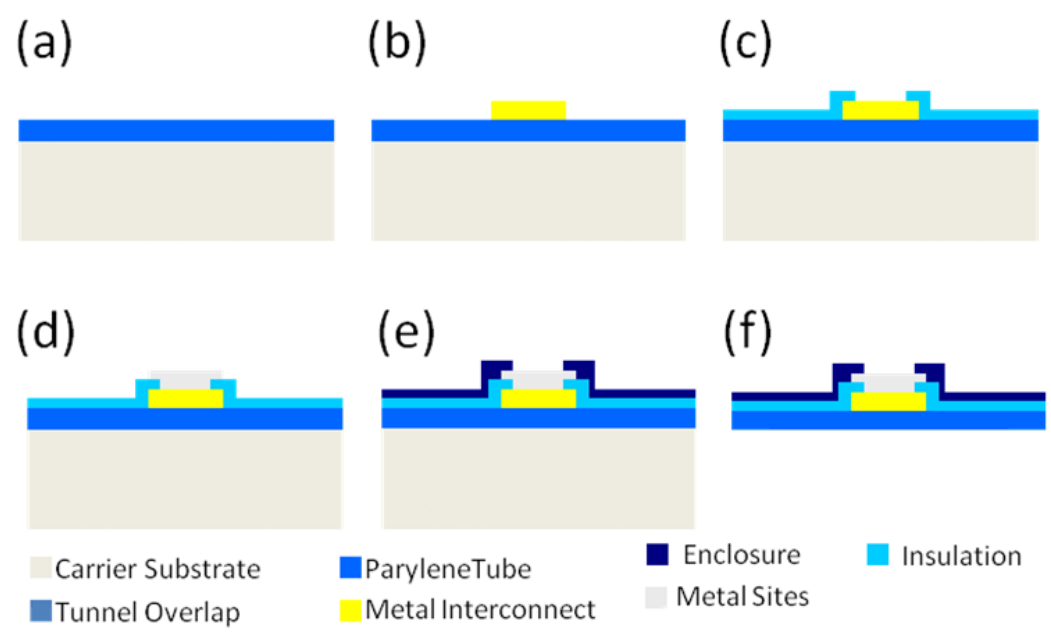

Fig. 2.27: Process flow for the fabrication of parylene electrode arrays. (a) a substrate layer of parylene is vapor deposited on a silicon carrier, (b) $\mathrm{Cr}-\mathrm{Au}-\mathrm{Cr}$ interconnect is evaporated and defined with a TMAH liftoff technique, (c) a dielectric layer of parylene is vapor deposited over the interconnect following an oxygen plasma ash, and contact openings are etched, (d) Ti-Ir electrode sites are 
sputter deposited at low base pressures and removed with TMAH liftoff, (e) following an oxygen plasma ash, a top coat of parylene is applied to the arrays and is oxygen plasma etched to remove the parylene from the sites and field areas.

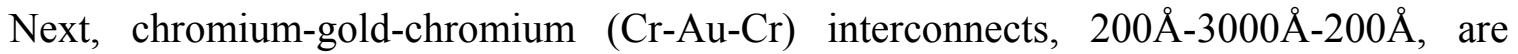
evaporated on this parylene base and patterned with acetone liftoff (Fig. 2.27b). Following the liftoff process, the wafers are pre-treated with an oxygen plasma ash (200W, 250mTorr). This ashing step serves to roughen, clean, and radicalize the bottom parylene layer before the vapor deposition of a $1 \mu \mathrm{m}$-thick dielectric layer of parylene. Following the dielectric deposition, $25 \mu \mathrm{m}$-wide by $100 \mu \mathrm{m}$-ong contact areas are etched over the lead traces with an oxygen plasma Reactive Ion Etch (RIE) (Fig. 2.27c). Then, Titanium-Iridium (Ti-Ir) sites having thicknesses of $500 \AA$ and $1500 \AA$, respectively, are sputter deposited and defined with acetone lift-off (Fig. 2.27d), followed by another ashing step. A final layer of parylene is then vapor deposited and RIE etched to open the sites and the field areas (Fig. 2.27e). As a last step, the arrays are released from the silicon carrier in $2 \% \mathrm{KOH}$ (Fig. 2.27f) and then heated in a nitrogen-purged vacuum oven for 12 hours.

Using the above process, parylene arrays were successfully developed for use in cat and guinea pig animal models with process yields of (Fig. 2.28, Fig. 2.29, Fig. 2.30). These arrays have tight lead pitches as low as $10 \mu \mathrm{m}$ on a parylene substrate, consistent with a 64-site human array with a single interconnect level. High site densities were achieved with center-to-center site spacings of $250 \mu \mathrm{m}$, consistent with a 128-site human array fed from two layers of interconnect. Array yields were above $80 \%$ per fabrication run. 


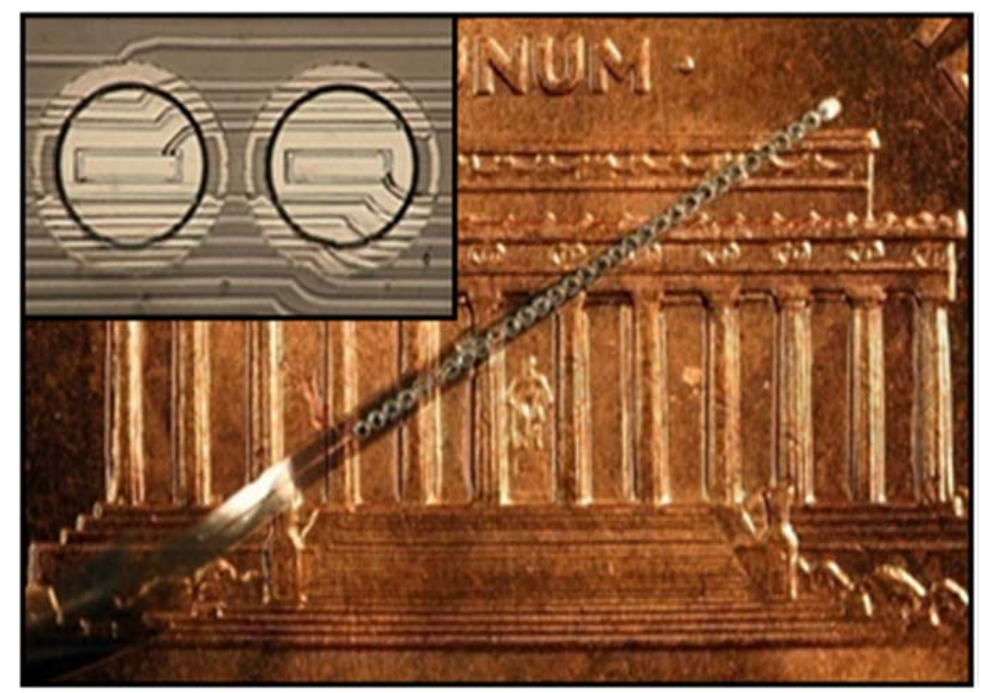

Fig. 2.28: A 32-site parylene guinea pig array with close-up of two Ti-Ir sites.

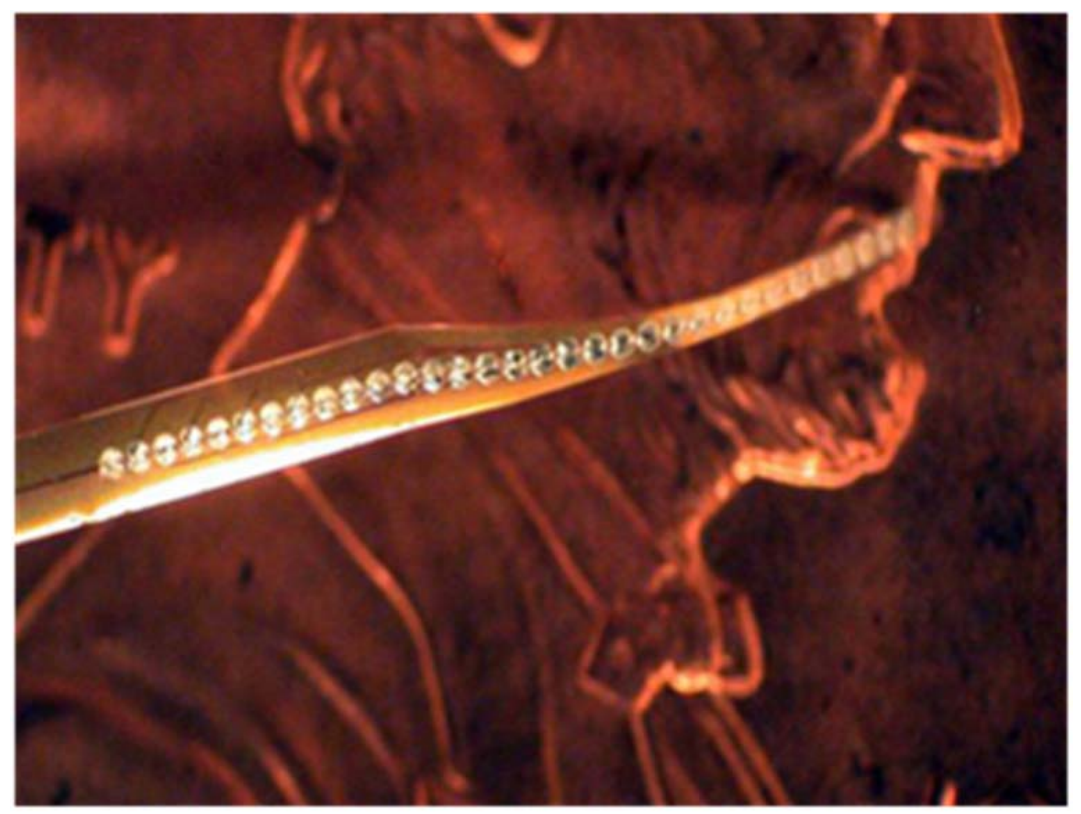

Fig. 2.29: A 32-site parylene cat array. 


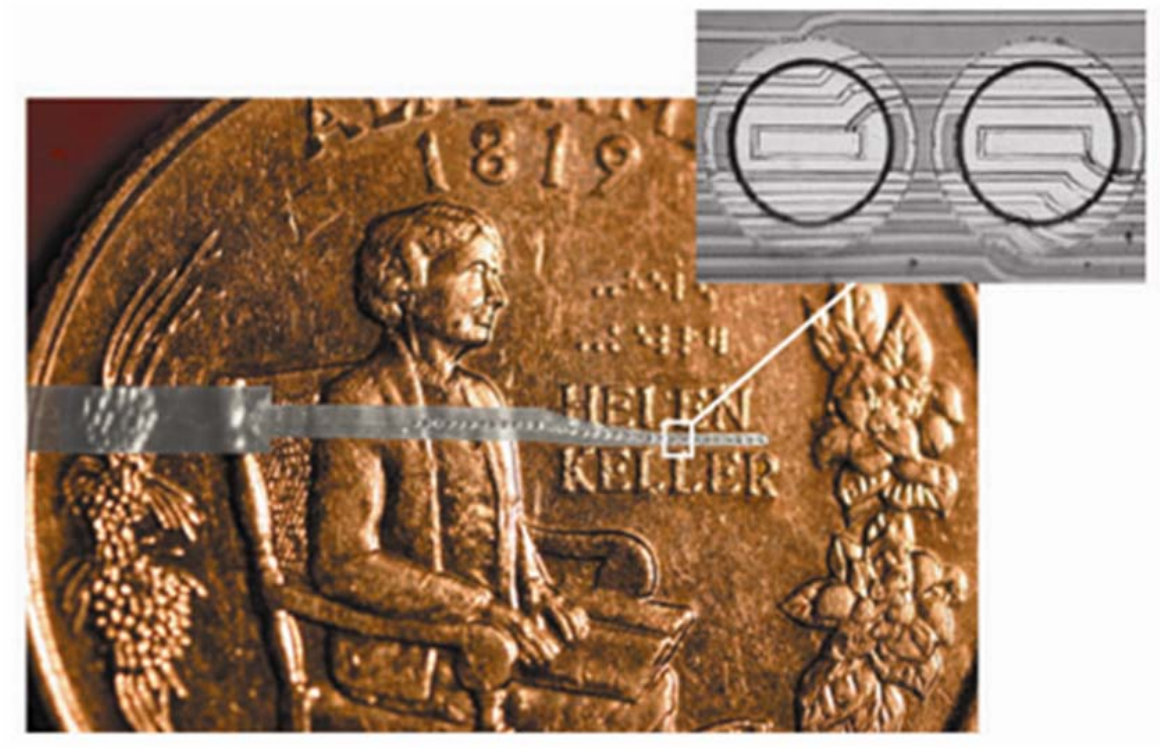

Fig. 2.30: A parylene substrate cat array with a close-up of two Ti-Ir sites.

\subsection{Array Assembly}

Before the arrays can be used for in-vitro or in-vivo tests, they must first be electrically assembled on Printed Circuit Boards (PCBs). The backends of the parylene cochlear arrays are compatible with rivet(ball)-bonding techniques [27]. Each area contact consists of a $25 \mu \mathrm{m} \mathrm{Cr}-\mathrm{Au}-\mathrm{Cr}$ frame partially encased in parylene (Fig. 2.31, Fig. 2.32). The frame has an outer diameter of $100 \mu \mathrm{m}$ and an inner diameter of $50 \mu \mathrm{m}$. An $80 \mu \mathrm{m}$-wide square of parylene is etched from the top surface of the contact pad to expose a portion of the $\mathrm{Cr}-\mathrm{Au}-\mathrm{Cr}$ metal for ball-bonding.
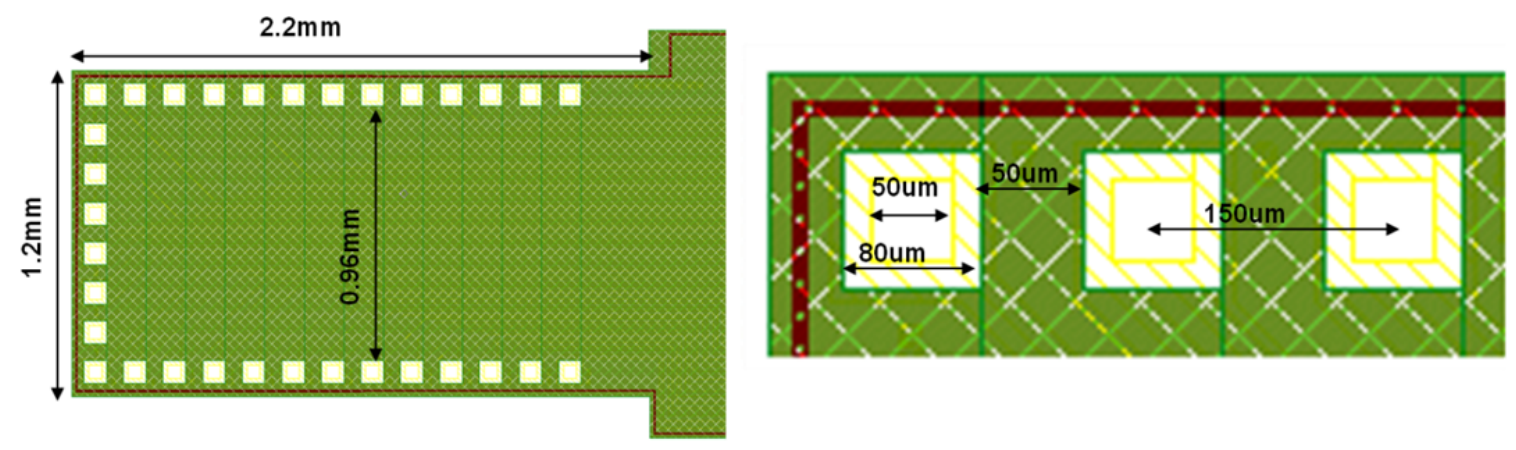

Fig. 2.31: An array backend and close-up of the ball bond area. 


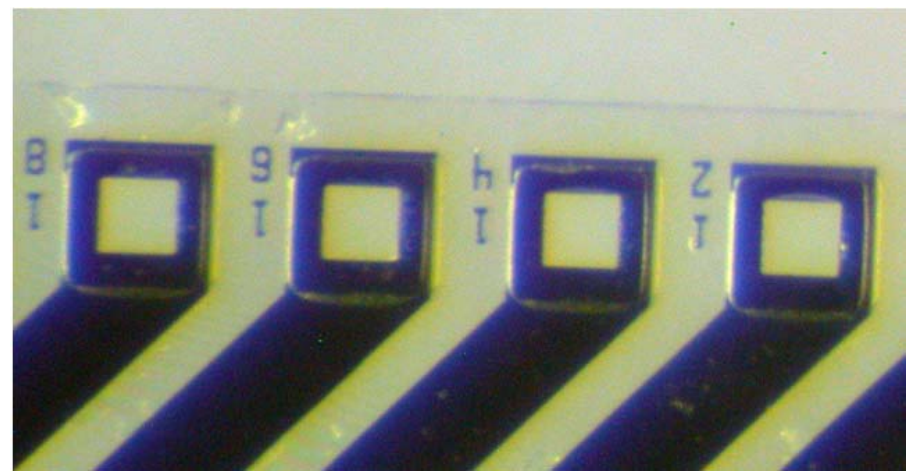

Fig. 2.32: Contact pads for ball bonding to the PCB. The exposed metal frame through the parylene allows threading of the ball bond to the PCB.

To assemble the arrays to the boards, the backends are themosonically ballbonded to the gold contact pads on a 32-trace PCB. In each bond, the gold ball of the bonder is centered over the $50 \mu \mathrm{m}$ inner diameter of the contact pad and makes contact with the $50 \mu \mathrm{m}$-wide gold trace of the PCB below it. After all thirty-two sites are bonded, Nusil MED4011 silicone is coated over the bonded backend, and the entire assembly is place in an $80^{\circ} \mathrm{C}$ oven for $1 \mathrm{hr}$ to allow the silicone to cure (Fig. 2.33).

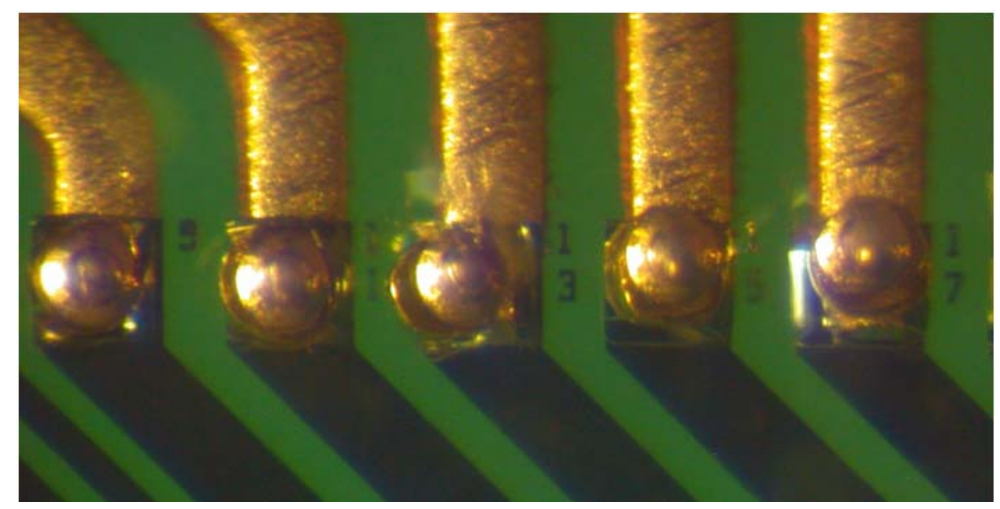

Fig. 2.33: Ball bonds going through contact frames on the array backend and connecting to the PCB.

\subsection{Electrical Performance}

Measuring the impedance of electrode sites is a good way to assess the yield of each fabrication run. Following assembly, the impedances of individual sites across each array were measured. The impedances varied depending on process and assembly outcomes. The lowest impedance values achieved across an array were under $0.5 \mathrm{k} \Omega$ and resulted from good Ti-Ir site adhesion, clean sites, and good bond contacts (Fig. 2.34). 
Sites at the tip of the array (sites 28 - 32) are closer to the edge of the wafer during processing and tend to have higher impedances than the sites numbered below them. When impedances were greater than $0.5 \mathrm{k} \Omega$, but less than $30 \mathrm{k} \Omega$ this typically indicated that there was some residual porous parylene on the electrode site, or poor bond contacts (Fig. 2.35). For some sites, their impedances would initially be high, but would drop down to just few hundred ohms after cyclic voltammetry. It is likely that these occurrences were a result of residual scum left on the sites post-processing that was easily removed with the application of voltage. High site impedances (in the meg-ohm range) were typically a result of fractured sites, sites still covered with a uniform coating of parylene, broken interconnect lines, or broken bond sites.

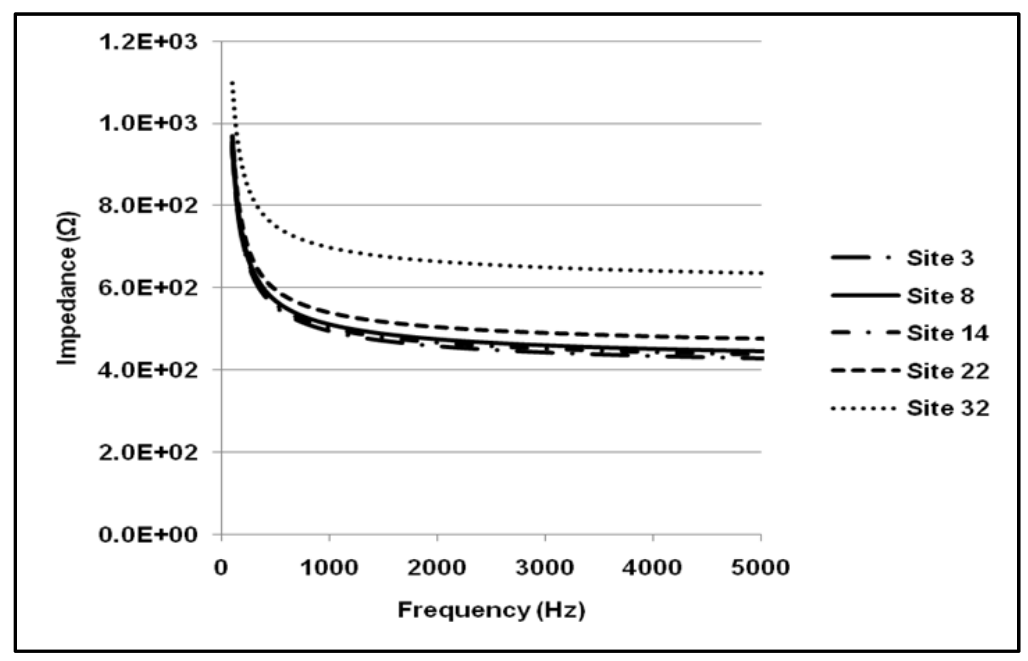

Fig. 2.34: Impedances of five sites across an array as a function of frequency (pre-activation). For simplicity's sake, only five electrode impedances are shown. They are representative of impedances in neighboring sites as well. 


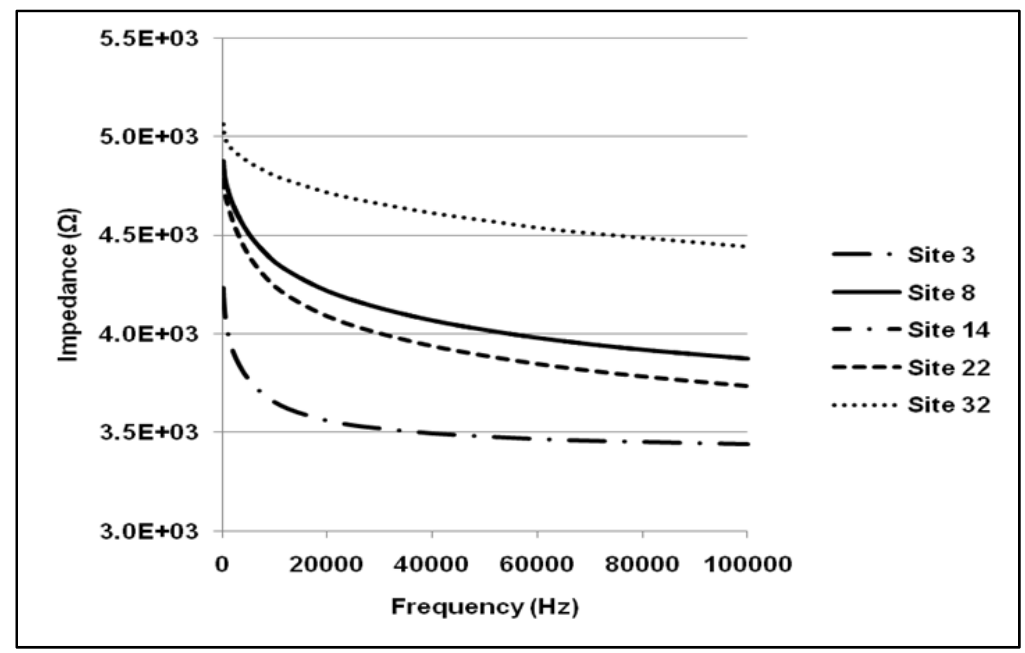

Fig. 2.35: Impedances of five electrode sites across a parylene array as a function of frequency (pre-activation). For simplicity's sake, only five electrode impedances are shown. They are representative of impedances in neighboring sites as well.

In addition to measuring impedances immediately after processing to determine yield, the impedance and charge capacity of each site on the arrays were measured after activation but before implantation. These measurements were done to ensure safe charge delivery and assess the success of activation as well as to characterize the initial state of each electrode before implantation. Knowing this initial state gave more insight into array placement inside the cochlea, the stimulation response of the animals, and any mechanical damage done to the sites as a result of insertion.

An anodically-formed IRidium Oxide Film (AIROF) was grown on the Ir surface as discussed in section 2.1.2. Activation was done using 200 cycles of a triangle waveform that swept the sites from $-0.6 \mathrm{~V}$ to $0.8 \mathrm{~V}$ at a rate of $100 \mathrm{mV} / \mathrm{s}$, relative to a $\mathrm{Ag} / \mathrm{AgCl}$ electrode. The cyclic voltametry was carried out in a solution of $1 \mathrm{X}$ PBS buffer with a $\mathrm{pH}$ of 7.4 . It was comprised of $8 \% \mathrm{NaCl}, 0.2 \% \mathrm{KCL}, 1.44 \% \mathrm{Na} 2 \mathrm{HP} 04$ and $0.24 \%$ KH2PO4. The electrode sites were activated to charge densities of $2000 \mu \mathrm{C} / \mathrm{cm}^{2}$ (Fig. 2.36, Fig. 2.37). 


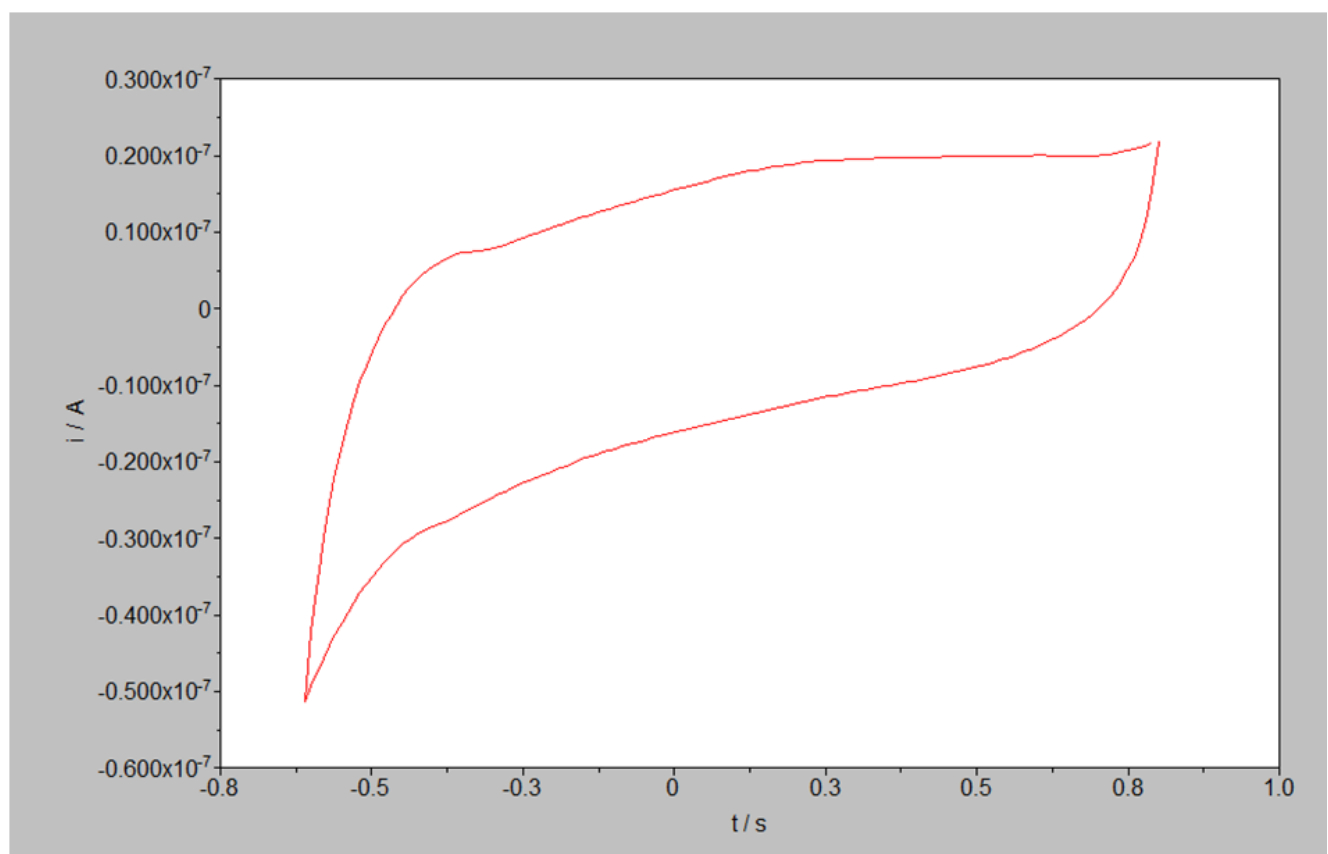

Fig. 2.36: Ir site pre-activation. $\mathrm{CV}$ sweeps from $-0.6 \mathrm{~V}$ to $0.8 \mathrm{~V}$ at a rate of $100 \mathrm{mV} / \mathrm{s}$. The $\mathrm{x}$-axis is time (s) and the $\mathrm{y}$-axis is current (A).

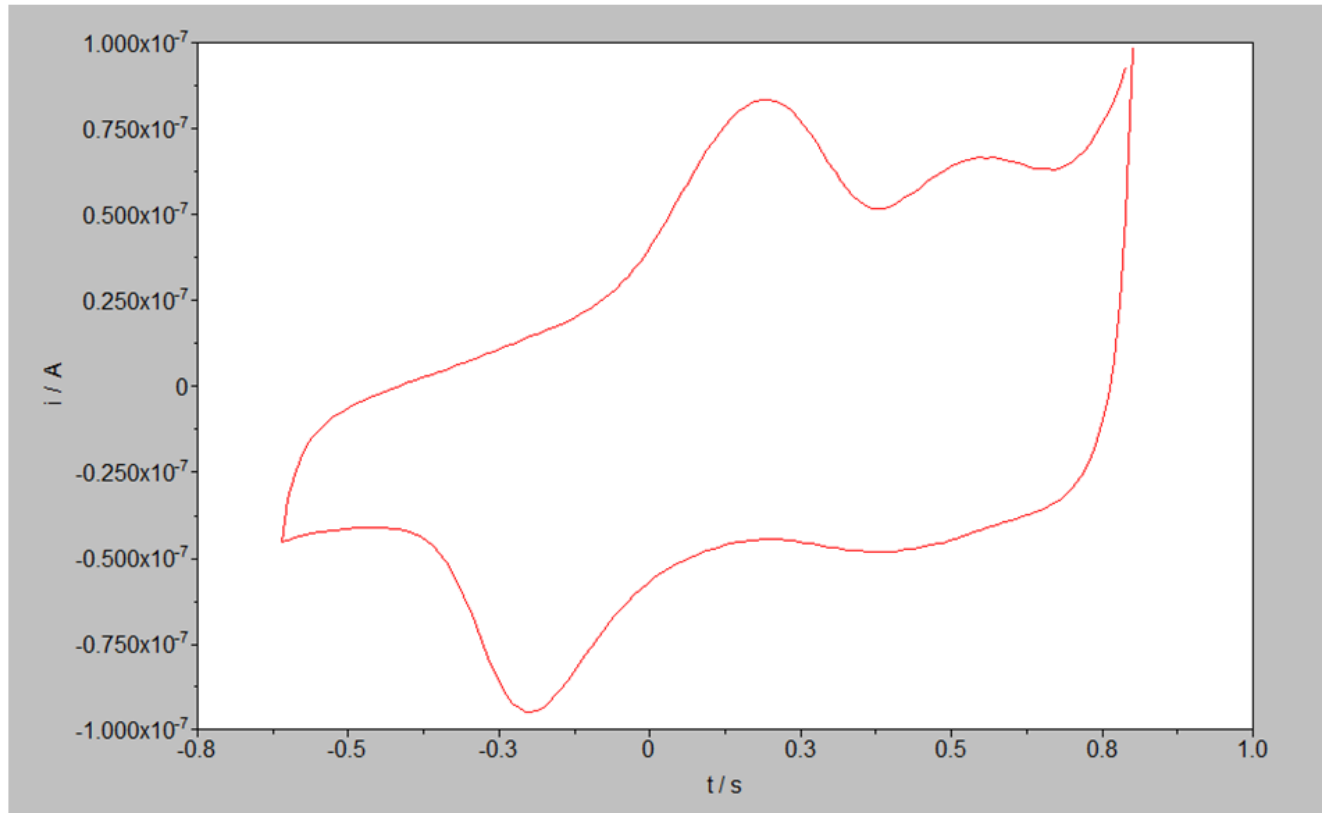

Fig. 2.37: IrO electrode site, post-activation. Site was activated to a charge capacity of $0.6 \mu \mathrm{C}$. The $\mathrm{x}$-axis is time (s) and the $\mathrm{y}$-axis is current (A). 


\subsection{Mechanical Performance}

To test the flexibility and robustness of the parylene arrays, we conducted bending and twisting flex-tests on the parylene cables. These cables were fabricated using a variation of the process outlined in section 2.4. Fabrication began with the vapor deposition of a $5 \mu \mathrm{m}$-thick layer of parylene on a silicon carrier wafer. Next, chromiumgold-chromium (Cr-Au-Cr) interconnects, $200 \AA-3000 \AA-200 \AA$, were evaporated on this parylene base and patterned with acetone liftoff. Following the liftoff process, the parylene was pre-treated with an oxygen plasma ash (200W, 250mTorr). A final layer of parylene was then vapor deposited and oxygen plasma etched to open the bond pad sites at the backend and the field areas. As a last step the cables were released from the silicon carrier in $2 \% \mathrm{KOH}$ (Fig. 2.3f). The released cables were ball-bonded to printed circuit boards using thermosonic bonding [28].

These experiments looked at both the robustness of the parylene and the robustness of the interconnect lines. Cable impedances were recorded as they were bent or twisted to observe any fractures that might arise in the lead lines. Impedances between the cable and the saline solution were recorded pre- and post-flexing to observe any signs of parylene cracking. For both experiments, a test setup was built using a Labviewcontrolled stepper motor. The setup for the bending experiments contained both a movable arm and a fixed arm. The cable was bonded to Printed Circuit Boards (PCBs) that were connected to the movable and fixed arms. One end of the cable was connected to a moveable arm which was driven by a stepper motor to bend it about a $\sim 2 \mathrm{~mm}$ radius pole. The other end of the cable was attached to the fixed arm (Fig. 2.38). In one complete cycle, the cable is bent a set number of degrees and then unbent back to the starting position. 


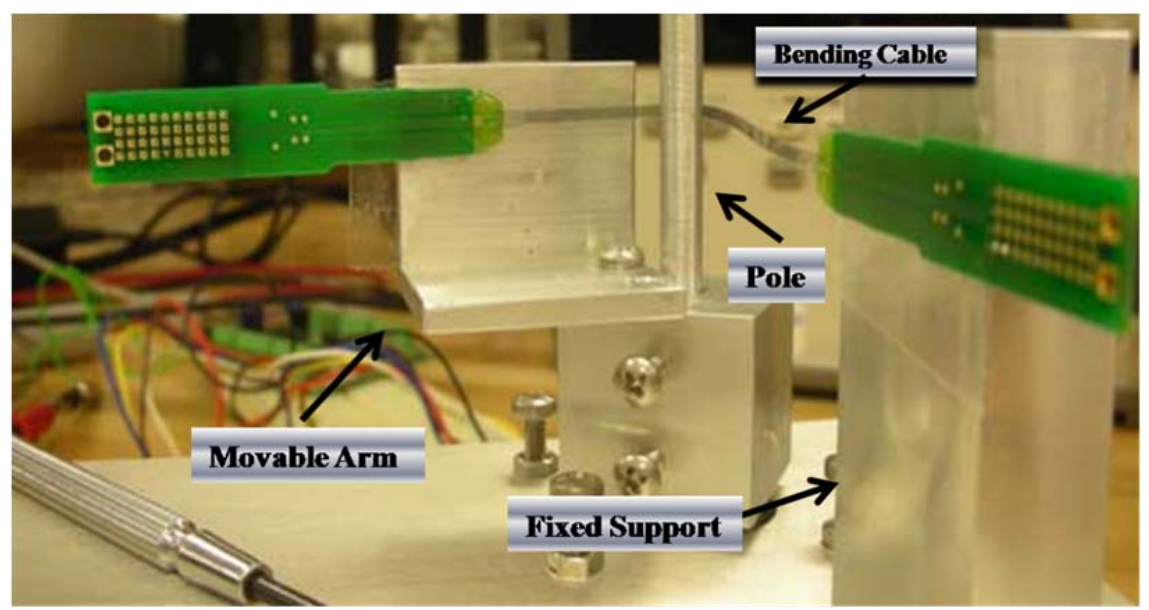

Fig. 2.38: Experimental set-up for cable bend-tests.

The setup for the twisting experiment rotates the motor driven arm in relation to the fixed support, and twists the cable (Fig. 2.39). In one complete cycle, the cable is twisted a set number of degrees and then twisted back to the starting position.

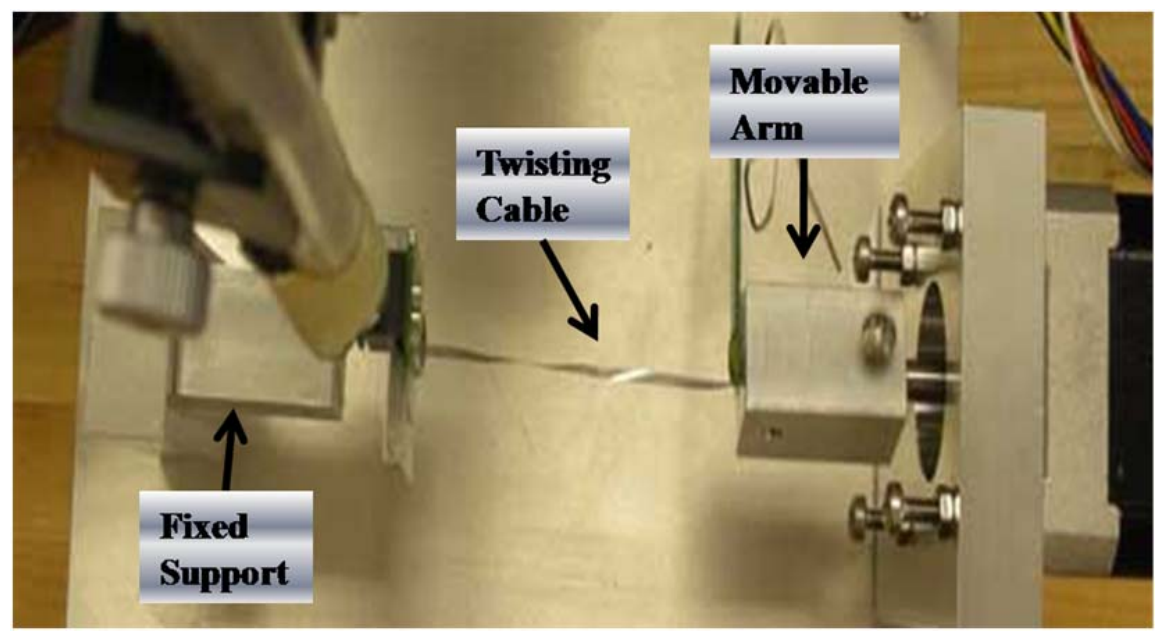

Fig. 2.39: Experimental set-up for cable twist-tests.

For both test set-ups, the stepper motor was controlled by a LabView interface (Fig. 2.40). The interface is used to set the velocity, angle of bending/twisting, and total number of bends/twists. It records the cable impedances during extended flex cycles using a $1 \mathrm{kHz}$ sinusoidal voltage input. 


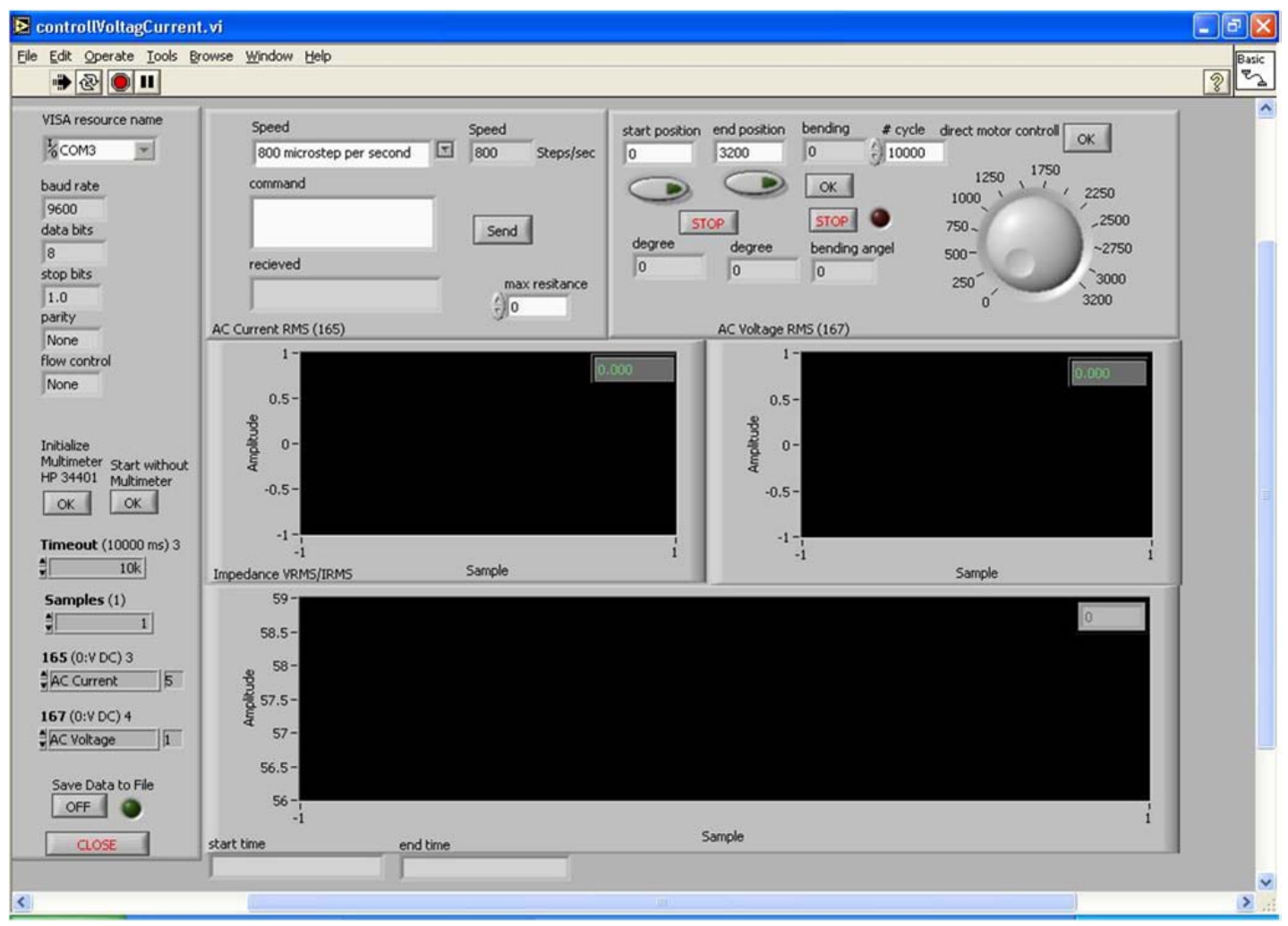

Fig. 2.40: Labview interface for setting flex-test parameters and recording impedance data.

On the far left hand side of the front panel are the initialization settings for the virtual serial port connection between the PXI-computer and the motor control unit. Below this are the settings for two HP34401A multimeters, which record the $1 \mathrm{kHz}$ voltage and current through the cable. In the upper part of the interface window there is a panel for controlling the motor speed of bending/twisting. Next to this, is a panel where the degree of twisting and bending angle can be set as well as the number of cycles. There is also a turn dial for manual control of bending/twisting. The "number of cycle" control allows the user to create a terminating condition for the bending/twisting experiments.

We conducted flex tests in which one cycle of bending consisted of the cable being bent $90^{\circ}$ forward and then back straight. One complete cycle of twisting consisted of the cable being twisted forward $360^{\circ}$, back to $0^{\circ}$, backwards $-360^{\circ}$, and ending back at $0^{\circ}$. Seven thousand bending cycles, at $4.5 \mathrm{~s}$ per cycle, were performed on individual cables and no significant drop in impedance was observed (Fig. 2.41). Thirty-five hundred twisting cycles on individual cables, at $18.25 \mathrm{~s}$ per cycle, also did not lead to any 
decline in impedance (Fig. Fig. 2.42). This indicates that the lead lines have suitable robustness for insertion into the helical cochlea. Note that in the graphs the initial drop in impedance is a result of the test set-up, and similar behavior was seen when the set-up was used to measure an off the shelf $50 \Omega$ resistor.

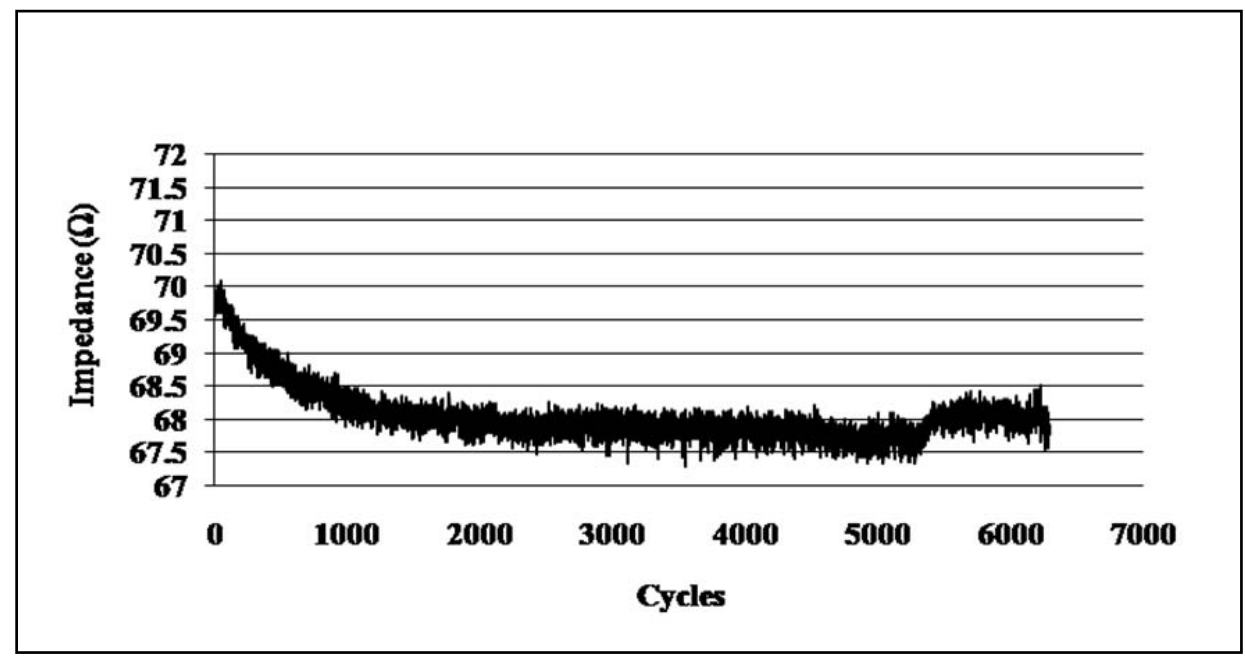

Fig. 2.41: Impedance of cable leads versus the number of bend-cycles. Impedance measurements were made during array bending. The impedance does not significantly drop with bending. Drop within the first 1000 cycles can be attributed to test set-up.

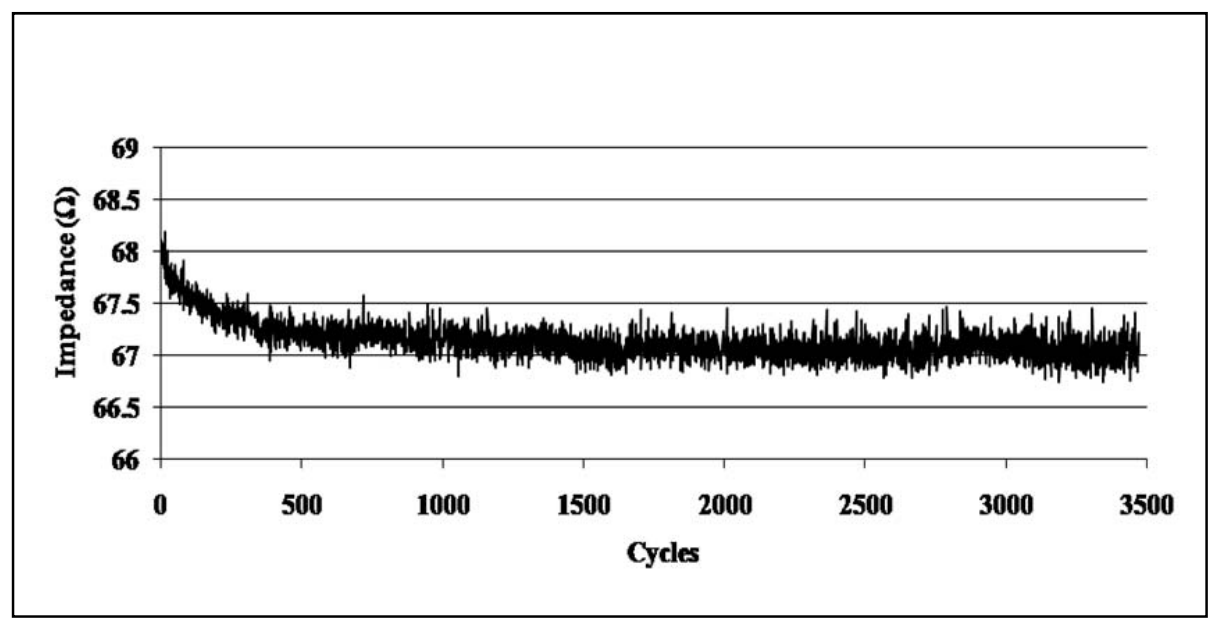

Fig. 2.42: Impedance of cable leads versus the number of twist-cycles. Impedance measurements were made during array twisting. The impedance does not significantly drop with twisting. Drop within the first 500 cycles can be attributed to test set-up. 
The saline submersion experiments were conducted by placing the cable in saline solution $\left(8 \mathrm{~g} \mathrm{NaCl}\right.$ in $\left.11 \mathrm{H}_{2} 0\right)$ up to the point where it connects to the PCB, and recording its impedance to the solution both before and after flexing. The impedances in saline between the lead lines and the solution were measured before and after 5000 cycles of twisting. First, the cable was soaked in the saline solution for 60 minutes and measured; then it was removed from the saline solution and subjected to the twist-tests; afterwards it was again soaked in saline for 60 minutes and re-measured. The impedances to the solution did undergo a decline after twisting (Fig. 2.43). However, the declines were smaller than what would be seen if the parylene was cracking significantly, and are not significant enough to effect array stimulation or recording. The drops in impedance could be attributed to pinholes in the parylene substrate, generated during deposition steps.

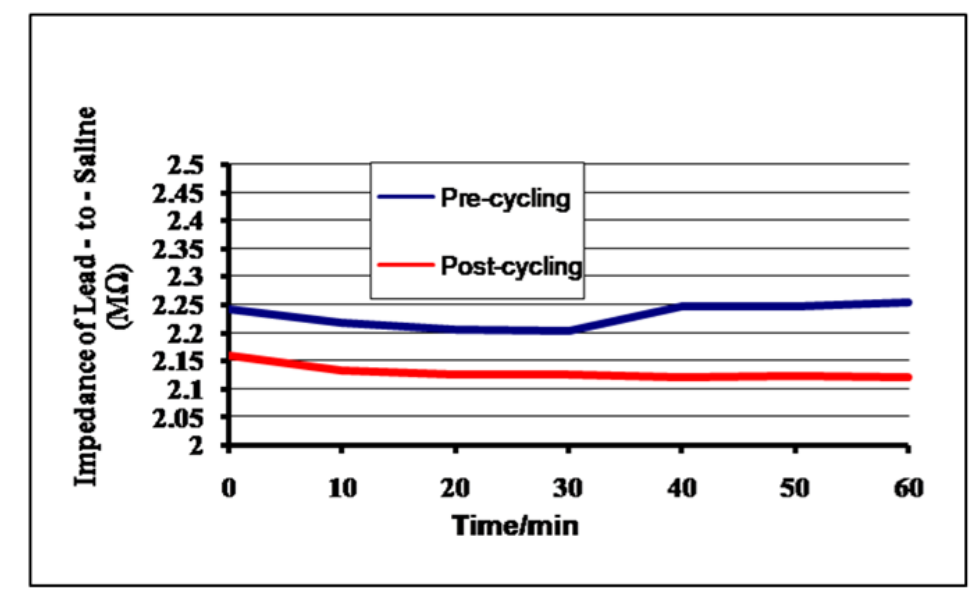

Fig. 2.43: The impedances between the cable lead lines and the saline solution over time. There is no significant difference in impedance pre- and post-twisting. Above graph is representative of typical results seen across multiple cable trials.

\subsection{Stimulating Array Summary}

A parylene cochlear electrode stimulating array has been developed for use as a cochlear implant. The array is comprosed entirely out of biocompatible polymer and metal layers, with $18 \mu \mathrm{m}$-diameter sites spaced $250 \mu \mathrm{m}$ center-to-center, with a $10 \mu \mathrm{m}$ lead pitch. Smooth non-cracked Ti-Ir sites have been successfully deposited onto the parylene substrate. Final devices yields are above $80 \%$. Flex tests on parylene cables 
indicate that the devices are robust enough to survive insertion into tightly-pitched helical animal and human cochleas. The site-to-contact resistances of the parylene arrays can be as low as $\sim 0.4 \mathrm{k} \Omega$ when measured in PBS. The IrO sites have been activated to charge capacities of $2000 \mu \mathrm{C} / \mathrm{cm}^{2}$ with no site delamination from the parylene substrate. With the addition of backings, it is expected that the arrays will achieve lower thresholds than the silicon devices, since their added robustness and flexibility will aid in deeper insertion and closer placement to the modiolus wall.

\subsection{References}

[1] Jianbai, W. ; Gulari, M. N. ; Wise, K. D., "A Parylene-Silicon Cochlear Electrode Array with Integrated Position Sensors,", $28^{\text {th }}$ IEEE International Conference of Engineering in Medicine and Biology Society, pp. 3170-3173.

[2] Bhatti, P.T., "A high-density thin-film electrode array for a cochlear prosthesis," Electrical Engineering and Computer Science, Ph.D. Ann Arbor: The University of Michigan, 2006

[3] Specialty Coating Services, "SCS Parylene Properties", Aug. 2011, http://www.scscoatings.com/index.aspx

[4] Para Tech Coating Inc., "Parylene Technology", Aug. 2011, http://www.parylene.com/technology/specifications-properties.html

[5] Jui-Mei Hsu; Rieth, L.; Normann, R.A.; Tathireddy, P.; Solzbacher, F.; , "Encapsulation of an Integrated Neural Interface Device With Parylene C," IEEE Transactions on Biomedical Engineering, vol.56, pp.23-29, 2009

[6] Suzuki, Y.; Yu-Chong Tai; , "Micromachined high-aspect-ratio parylene beam and its application to low-frequency seismometer", $16^{\text {th }}$ IEEE International Conference on Micro Electro Mechanical Systems, pp. 486- 489, 2003

[7] Rodger, D.C.; Weiland, J.D.; Humayun, M.S.; Yu-Chong Tai; , "Scalable flexible chip-level parylene package for high lead count retinal prostheses,", 13th IEEE International Conference on Solid-State Sensors, Actuators and Microsystems , vol.2, pp. 1973- 1976, 2005

[8] Para Coat Technologies, Aug. 2011, http://pctconformalcoating.com/ParyleneConformalCoatingTechnology.html

[9] Weiland, J.D.; Anderson, D.J., "Chronic Neural Stimulation with Thin-Film Iridium Oxide Electrodes.", IEEE Transactions on Biomedical Engineering, vol.47, pp. 911- 918, 2000 
[10] J. D. Weiland, "Electrochemical properties of iridium oxide stimulating electrodes," in Biomedical Engineering, Ph.D. Thesis, Ann Arbor: The University of Michigan, 1997

[11] Voie, A. H.; Spelman, F.A., “ Three-Dimensional Reconstruction of the Cochlea from Two-Dimensional Images of Optical Sections.”, Computerized Medical Imaging and Graphics, pp. 377-384,1995

[12] Hatsushika, S.I; Shephard, R. K.; Tong, Y.C.; et. al., "Dimensions of the Scala Tympani in the Human and Cat With Reference to Cochlear Implants.", Ann Otol Rhinol Laryngol. vol. 99, pp. 871-876, 1990

[13] Beebe, X.; Rose, T.L.; "Charge Injection Limits of Activated Iridium Oxide Electrodes with $0.2 \mathrm{~ms}$ Pulses in Bicarbonate Buffered Saline (Neurological Stimulation Application).", IEEE Transactions on Biomedical Engineering, vol. 36, pp. 494-495, 1998

[14] Kim, C., "A 64-Site Multiplexed Low-Profile Neural Probe for Use in Neural Prosthesis." , in Electrical Engineering and Computer Science, Ph.D. Ann Arbor: University of Michigan, 1994

[15] Walsh, K.; Norville, J.; Yu-Chong Tai; , "Photoresist as a sacrificial layer by dissolution in acetone," 14th IEEE International Conference on Micro Electro Mechanical Systems , pp.114-117, 2001

[16] Williams, K.R.; Gupta, K.; Wasilik, M.; , "Etch rates for micromachining processing-Part II," Journal of Microelectromechanical Systems, vol.12, pp. 761778,2003

[17] Liger, M.; Rodger, D.C.; Yu-Chong Tai; , "Robust parylene-to-silicon mechanical anchoring," $16^{\text {th }}$ IEEE International Conference on Micro Electro Mechanical Systems, pp. 602- 605, 2003

[18] Redd R.; Spak, M.A.; Sagan, J.P.; Lehar, P.O.; Ralph, R.D., "Lithographic process for high-resolution metal lift-off", Procceedings of SPIE, vol. 3678, pp. $641-651,1999$

[19] Seymour, J., “Advanced Polymer-Based Microfabricated Neural Probes Using Biologically Driven Designs.”, in Biomedical Engineering, Ph.D. Ann Arbor: University of Michigan, 2009

[20] Sharma, Ashok K.; Yasuda, H.; , "Effect of glow discharge treatment of substrates on parylene-substrate adhesion," Journal of Vacuum Science and Technology, vol.21, pp.994-998, 1982 
[21] Pornsin-Sirirak, N.; Liger, M.; Yu-Chong Tai; Ho, S.; Chih-Ming Ho; , "Flexible parylene-valved skin for adaptive flow control," $15^{\text {th }}$ IEEE International Conference on Micro Electro Mechanical Systems, pp.101-104, 2002

[22] Liston, E.M.; Martinu, L.; Wertheimer, M.R., "Plasma surface modification of polymers for improved adhesion: a critical review", Journal of Adhesion Science and Technology, vol. 7, pp. 1091-1127, 1993

[23] Huang, R.; Tai, Y.C.; , "Parylene to silicon adhesion enhancement," Solid-State Sensors, Actuators and Microsystems Conference, pp.1027-1030, 2009

[24] Kim, H.; Najafi, K.; , "Characterization of low-temperature wafer bonding using thin-film parylene," Journal of Microelectromechanical Systems, vol.14, pp. $1347-1355,2005$

[25] Rodgers, D.C., "Development of Flexible Parylene-Based Microtechnologies for Retinal and Spinal Cord Stimulation and Recording.", Ph.D. Pasadena:

Dissertation, California Institute of Technology, 2008

[26] Noh, H.S.; Moon, K.S.; Cannon, A; Hesketh, P.J.; Wong, C.P., "Wafer Bonding Using Microwave Heating of Parylene Intermediate Layers." Journal of Micromechanics and Microengineering, vol. 14, pp. 625-631, 2004

[27] Meyer, J.-U.; Stieglitz, T.; Scholz, O.; Haberer, W.; Beutel, H.; "High density interconnects and flexible hybrid assemblies for active biomedical implants," IEEE Transactions on Advanced Packaging, vol.24, pp.366-374, 2001 


\section{Chapter 3}

\section{ARRAY BACKING}

The backing of a cochlear electrode array is designed to give it the appropriate modiolus hugging, stiffness and positioning features. In commercial devices, backings are formed using injection molded silicone carriers. Previous thin-film arrays have used silicone carriers and hybrid thin-film backings (Table 1.1). Molded carriers for thin-film arrays increase their robustness, make them compatible with present surgical insertion tools and provide the fastest route to adoption by the medical device market. However, they are tedious to put in place on the microsized devices, are applied using a low throughput process, and are costly. In contrast, thin-film backings can be incorporated at wafer-level, allow for a high throughput, low cost batch fabrication, and can be readily enhanced with structures for visualizing implantation [1] and drug delivery. Both molded and thin-film backings were designed for use with the parylene arrays presented in this work. The design and results of both approaches are discussed in the following sections.

\subsection{Molded Backings}

Molded backings were investigated through collaborations with the University of California, San Francisco (UCSF), and Cochlear Ltd. The carriers generated in collaboration with UCSF are straight and stiff enough for direct placement inside the cochlea using tweezers. In collaboration with Cochlear Ltd., the parylene arrays were integrated into the Nucleus Contour Advance silicone carrier. Such carriers are precurved to hug the modiolus and are positioned using a flexible stylet wire that is removed from the array after insertion. 


\subsubsection{UCSF Silicone Carriers}

UCSF has a long history in the development of cochlear electrode arrays beginning with the early work of Merzenich and Loeb on multielectrode array development [2]. More recently, they have produced wire-bundle arrays for small animal implants [3]. These animal arrays are comprised of eight $200-300 \mu \mathrm{m}$ diameter Pt:Ir (90:10) alloy wires, injection molded with silicone (Fig. 3.1). A similar injection process was used for the parylene arrays with a specially-designed stainless steel mold (Fig. 3.2).

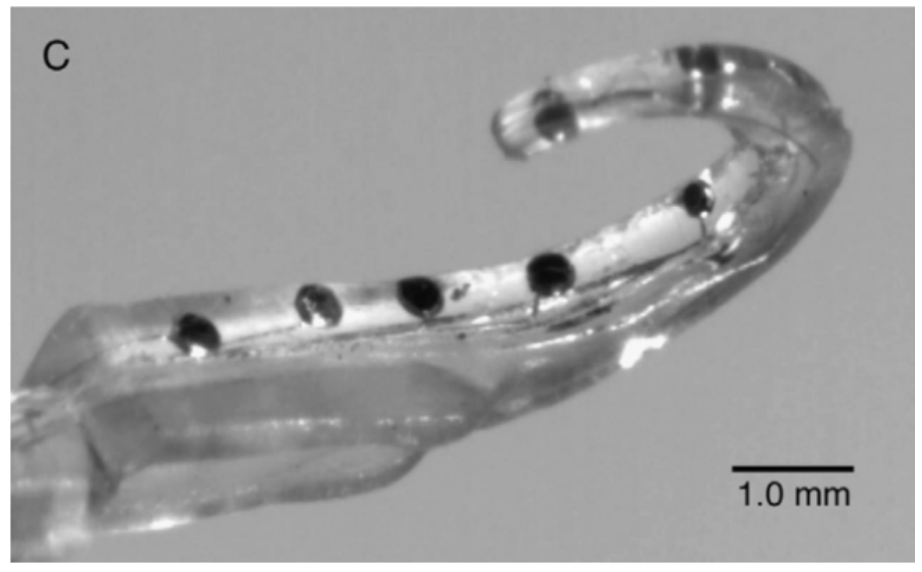

Fig. 3.1: The UCSF wire-bundle animal cochlear electrode array [3].
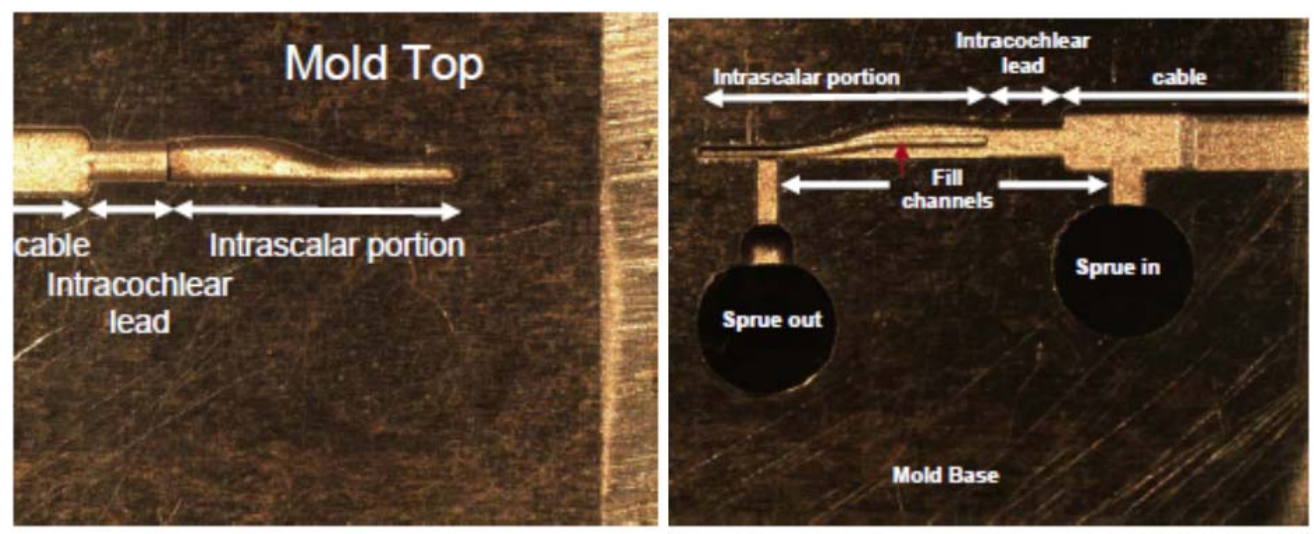

Fig. 3.2: The UCSF stainless steel mold used to injection-mold silicone around the Michigan parlyene cat arrays. 
The mold is sized to fit one turn of the cat cochlea and comes in two parts (upper mold, mold base). The mold base is $250 \mu \mathrm{m}$ tall and tapers from $1.1 \mathrm{~mm}$ to $400 \mu \mathrm{m}$, base - to - apex. Going down the center of the mold base is a raised ridge that is designed to contact the electrode sites and protect them from getting covered with silicone during the injection process (Fig. 3.3). This ridge also allows the silicone to overlap the edge of the array and mechanically lock it to the backing. The amount of overlap increases along the length of the array from $450 \mu \mathrm{m}$ to $150 \mu \mathrm{m}$, base - to - apex. The upper mold part is where the bulk of the silicone will be injected. It has a graded height which tapers 550 $\mu \mathrm{m}-250 \mu \mathrm{m}$, base - to - apex. The width of the upper mold is also tapered and goes from $1.3 \mathrm{~mm}-500 \mu \mathrm{m}$, base - to -apex.

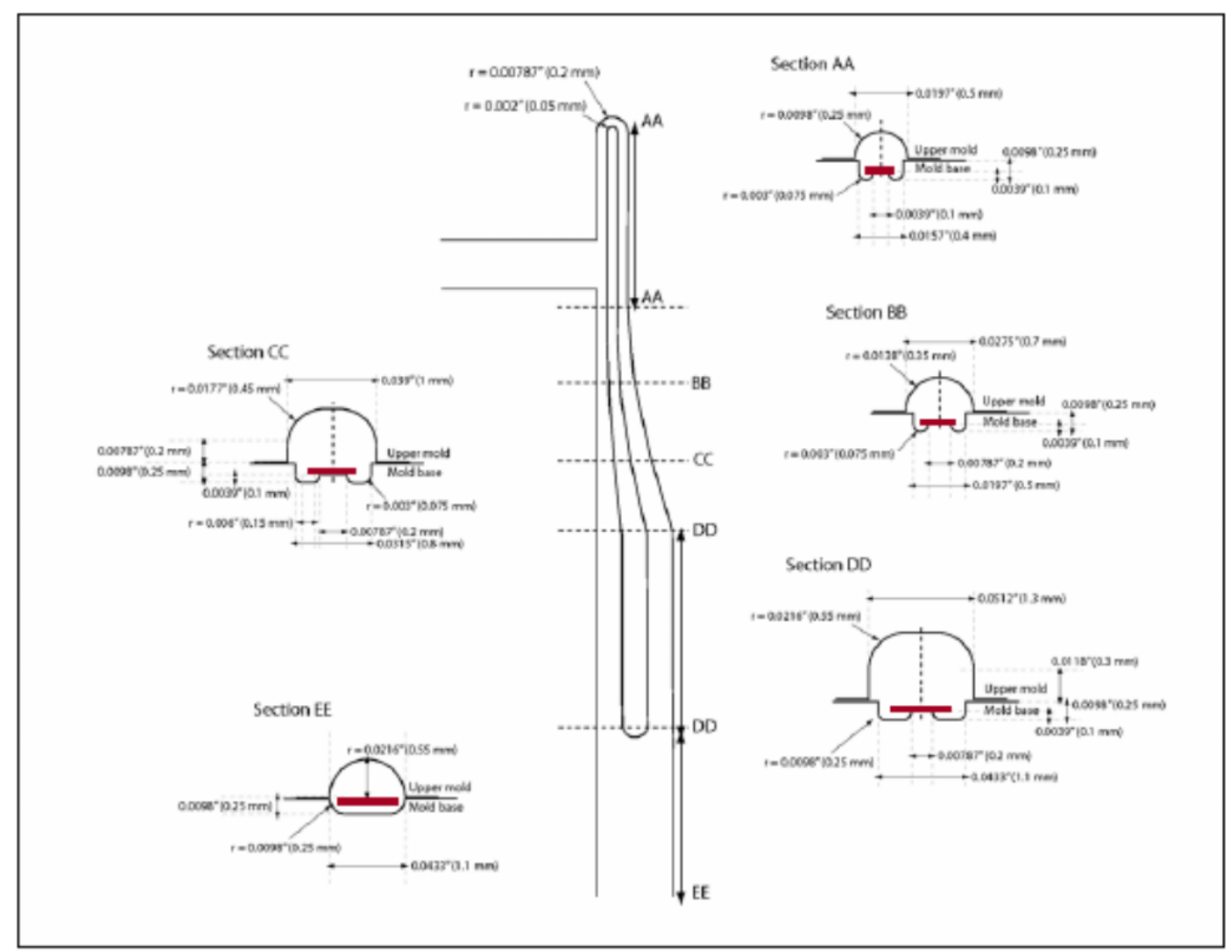

Fig. 3.3: Layout of the UCSF parylene array molding.

The molding process began with the application of dissolvable wax to the electrode sites. This wax was intended to secure the array to the ridge of the mold base 
and protect the sites from getting covered in silicone during the injection process. The wax coated arrays were then placed on the mold base, which was heated to $\sim 100^{\circ} \mathrm{C}$ for reflowing the wax. Once the arrays were fixed to the mold base, it was removed from the hot plate to cool and then attached to the upper mold. Degassed MED 4011 was injected through the mold inlet at 50psi, with excessive pressure build up alleviated by the outlet. As a last step, the entire assembly was placed in an oven at $\sim 110^{\circ} \mathrm{C}$ to cure the silicone.

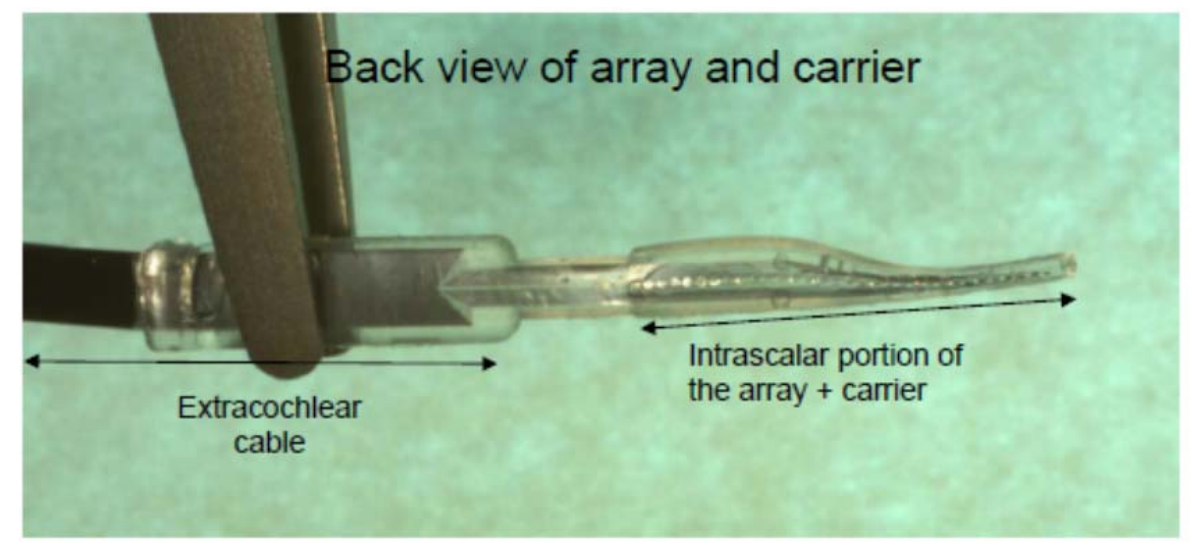

Fig. 3.4: Michigan parylene cat array in UCSF silicone carrier.

Several 32-site cat parylene cochlear arrays were molded using the above process, and the silicone carriers exhibited excellent adhesion to the parylene arrays (Fig. 3.4). However, there was only a $20 \%$ yield in active sites following the molding process. This decreased yield could have resulted from breakage or site occlusion during the molding process. To test the theory of interconnect or site damage as a result of physical stress on the array, impedances were recorded from the cured arrays pre- and post-removal from the stainless steel mold. Impedances before removal were just as high as the ones recorded after removal. This indicated that array breakage was not the cause of poor yield. Optical inspection further verified this conclusion. A second possibility was that the wax did not fully dissolve and occluded the sites with a residual scum. The wax was replaced with 9260 photoresist and the molding process adjusted. The new process consisted of coating a thin layer of resist in the bottom half of the mold. The mold is then placed on a hotplate at $110^{\circ} \mathrm{C}$ for 15 minutes to thoroughly rid it of solvents. The array is positioned over the resist while it is still on the hotplate, and then the entire base is 
removed from the heat and cooled to room temperature. After attaching the bottom and top mold halfs, degassed MED 4011 was injected through the mold inlet, and the entire assembly was placed in an oven at $\sim 110^{\circ} \mathrm{C}$ to cure the silicone. With this new proccesss, there was no appreciable difference in the impedance of sites recorded before and after the addition of the silicone carrier.

\subsubsection{Cochlear Ltd. Silicone Carriers}

Cochlear Ltd. is the leading manufacturer of cochlear implants and uses a proprietary injection molding process to produce their Nucleus 24 and Nucleus 24 contour arrays. In collaboration with Dr. Claudiu Treaba of Cochlear, silicone backings were added to the parylene arrays using the same proprietary molding process as that for the Nucleus Contour 24 outlined in section 1.3.4.

Several of the Michigan 32-site, cat parylene cochlear arrays were molded in Nucleus Contour carriers. The arrays were able to be successfully positioned in the molds and survived the injection process, something that could not be done with the silicon arrays of the past. Initial results show that it is possible to mold backings on the arrays, even in light of the fact that the Contour array molds are much larger than the parylene arrays. Additionally, the arrays showed good conformation to the silicone carriers in their curved state (Fig. 3.5). However, when the carriers were straightened using a stylet, the parylene substrates tended to delaminate from the backings and tear (Fig. 3.6). Strong silicone adhesives and mechanical anchors could be used to eliminate delamination. Breakage occurs due to mismatches in stiffness and elasticity between the thick silicone carrier and the thin parylene devices. Differences in stiffness could be adjusted for by modifying the thickness and overall size of the silicone backings. The thin parylene arrays do not require as much space as bundled wires, and as such, a reduction in mold size could be made. In addition to preventing breakage, the increased flexibility could also greatly decrease damage to residual hearing cells during surgical placement. 


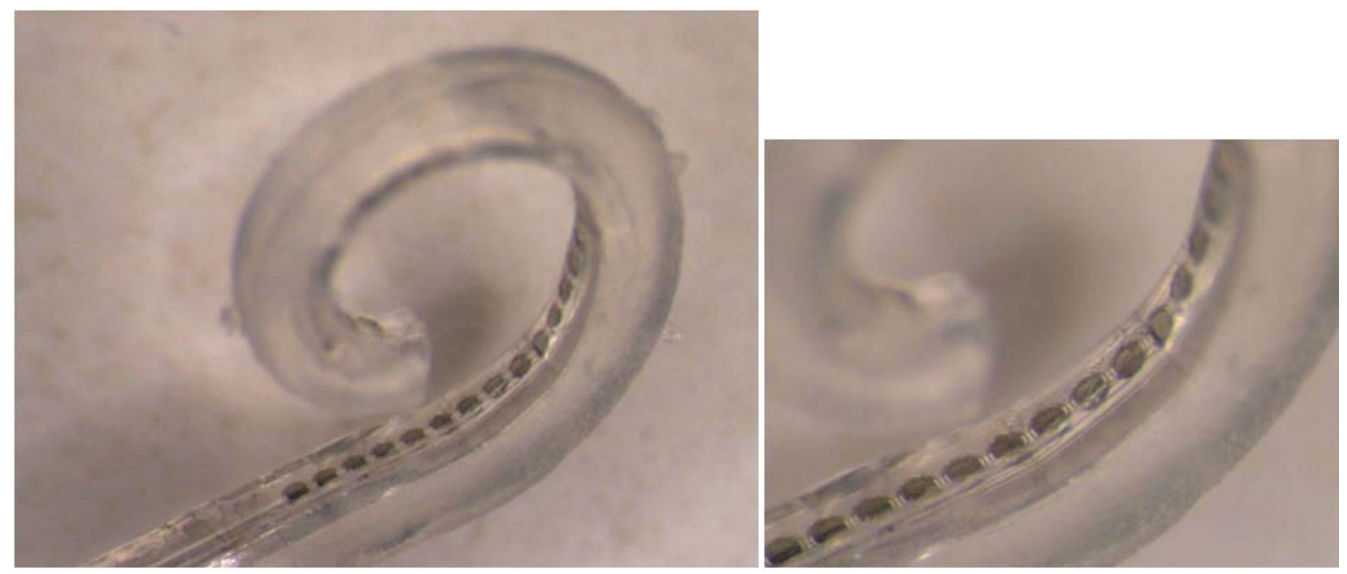

Fig. 3.5: Michigan parylene cat array in Cochlear Ltd. silicone carrier.

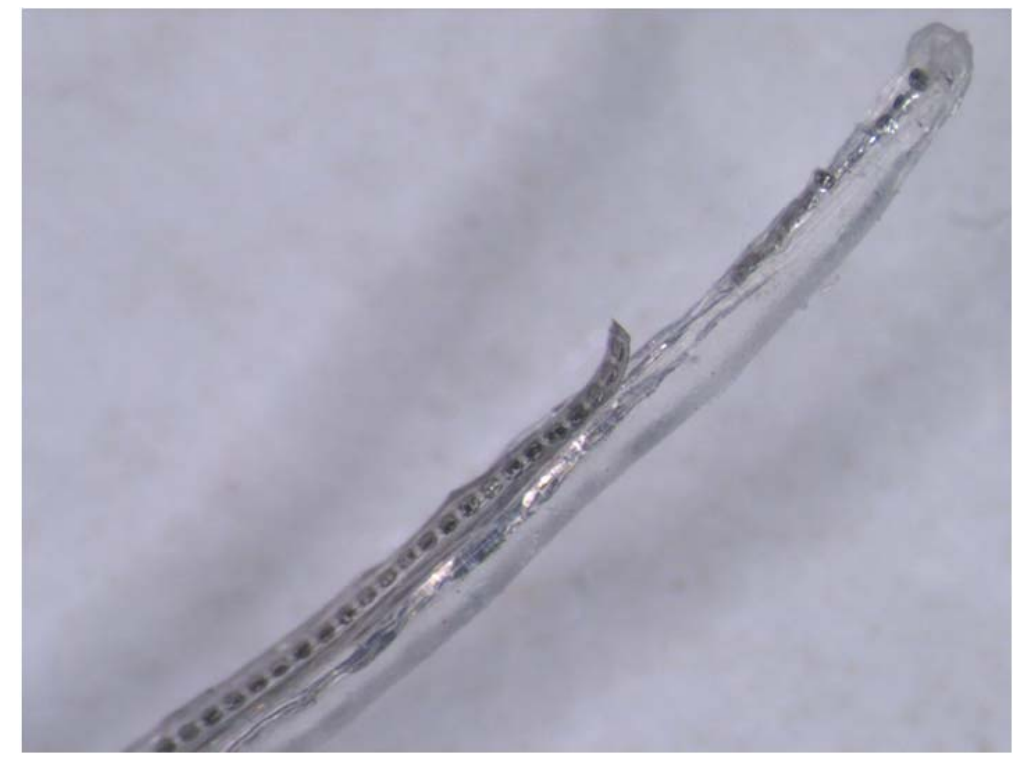

Fig. 3.6: Michigan parylene cat array in Cochlear Ltd. silicone carrier. The parylene array has delaminated from carrier when straightened with a stylet-wire.

\subsection{Thin-Film Backings}

One way of providing stiffness, modiolus hugging and positioning features into the parylene electrode arrays is through the addition of thin-film backings. Both hybrid and monolithic backings were investigated. Hybrid designs were investigated in collaboration with Michigan Technological University (MTU). These backings were incorporated with rigid layers to control stiffness, heat-formed curvature for modiolus hugging, and microtubes for stylet-based positioning. Monolithic backings were designed by us as an addition to the planar parylene arrays. The monolithic backings 
were built into the parylene arrays during processing and contained rings for stiffness control and stylet-based positioning as well as stressed polymer-metal layers for modiolus-hugging curl.

\subsubsection{Hybrid}

Michigan Technological University (MTU) has done work in the past to investigate PET thin-film backings for the Michigan silicon cochlear electrode arrays [4, 5] and more recently using Poly Lactic Acid (PLA) films [6].

The PET-actuated backings were made out of multichamber PET tubes, where pneumatic control of each chamber could provide articulated actuation and insertion [4]. The pneumatic tubes were comprised of three chambers (top, middle, and bottom) for controlling three separate segments of the array (basal, mid-section, and apical). Each chamber consisted of a PET tube $200 \mu \mathrm{m}$ in diameter with a $4 \mu \mathrm{m}$ wall thickness. The bottom chamber spanned the entire length of the array, the middle extended to two thirds of the array, and the top chamber covered the first third. Before getting stacked, the tubes were individually compressed and heated between two glass slides to give them a reduced, elliptical profile. Subsequent to flattening, the tubes were stacked and glued together using a biocompatible UV adhesive. The same adhesive was used to bond the silicon array to this stacked structure before wrapping the entire assembly around a spiral mandrel. In heating the array-wrapped mandrel, the PET could be heat formed in an oven at $110{ }^{\circ} \mathrm{C}-120{ }^{\circ} \mathrm{C}$ to take on a spiral shape. A modification of this backing scheme has been used by MTU to back the parylene electrode arrays.

To back the parylene arrays, a PET tube was attached to the parylene arrays and pre-curved for use with a stylet wire. At the base of the tube, a hypodermic steel tube was bonded to act as a guide for the stylet wire so that it could be steered straight and not punch through the wall of the PET tube during insertion. Additionally, a PET stiffener was added to the front side of the array cable (Fig. 3.7). 
Fig. 3.7: Assembled components of MTU PET backing for Michigan parylene arrays.

The assembly process begins with the flattening of a $365 \mu$ m-diameter PET tube with a wall thickness of $60 \mu \mathrm{m}$ using heat and compression as previously described. The width of the flattened tube is $500 \mu \mathrm{m}$. This flattened tube is glued to the backside of the parylene array shank with a biocompatible UV adhesive. At the basal end of the tube, a hypodermic steel tube with an inner diameter $200 \mu \mathrm{m}$ is attached. On the front side of the array cable, just beyond the shank, a stiffener made out of a PET sheet is attached. The first $11 \mathrm{~mm}$ of the entire assembly (array shank) is then wrapped around an Al mandrel having a diameter of $3.14 \mathrm{~mm}$. This diameter mandrel produces a curl in the array roughly equal to one full turn of the guinea pig cochlea. A straight-drawn stainless steel micro-wire, $210 \mu \mathrm{m}$ in diameter, is used as a stylet to uncurl the array for placement in the cochlea.

Several PET backed arrays were created (Fig. 3.8). As intended, the hypodermic steel tubes were successful in steering the stylet wires straight through the flattened PET tubes. The wires themselves were able to properly curl and uncurl the arrays upon insertion and removal. However, insertions of a stylet into an array resulted in plastic 
deformation of the heat-formed PET tubes (Fig. 3.9). For this reason, wires were only inserted once before array placement in the cochlea.

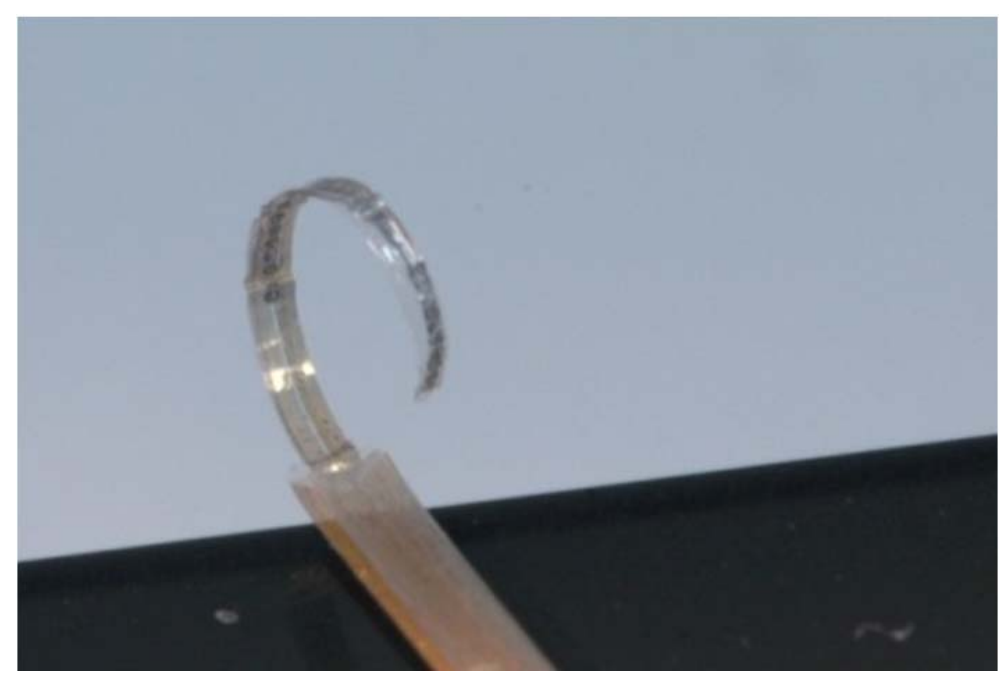

Fig. 3.8: Pre-curved MTU backing on Michigan parylene cat array.

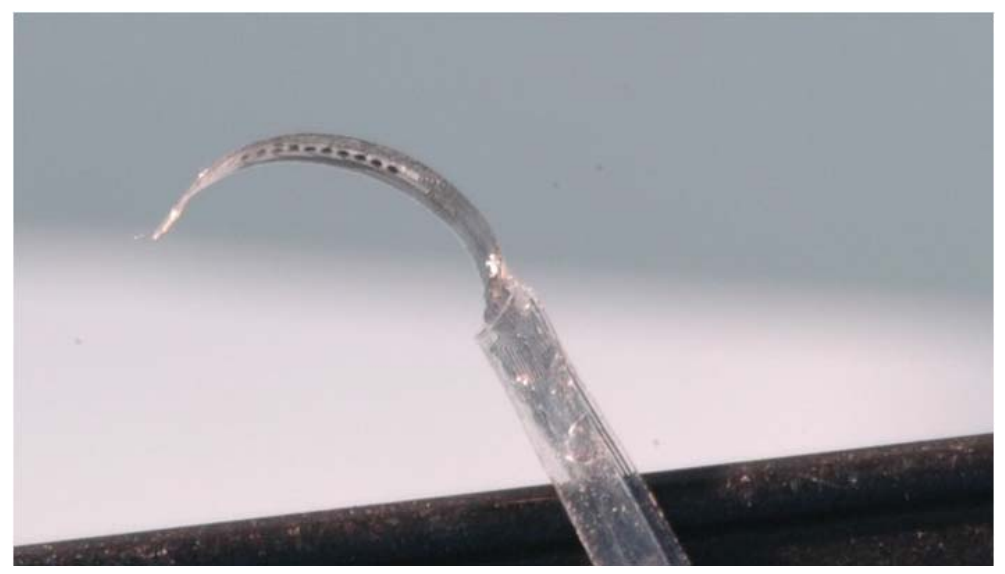

Fig. 3.9: Curvature of PET backing deformed as a result of multiple stylet-wire insertions.

Taking these precautions, insertions of the arrays were achieved with full placement of all sites into the cat cochlea (Fig. 3.10). 


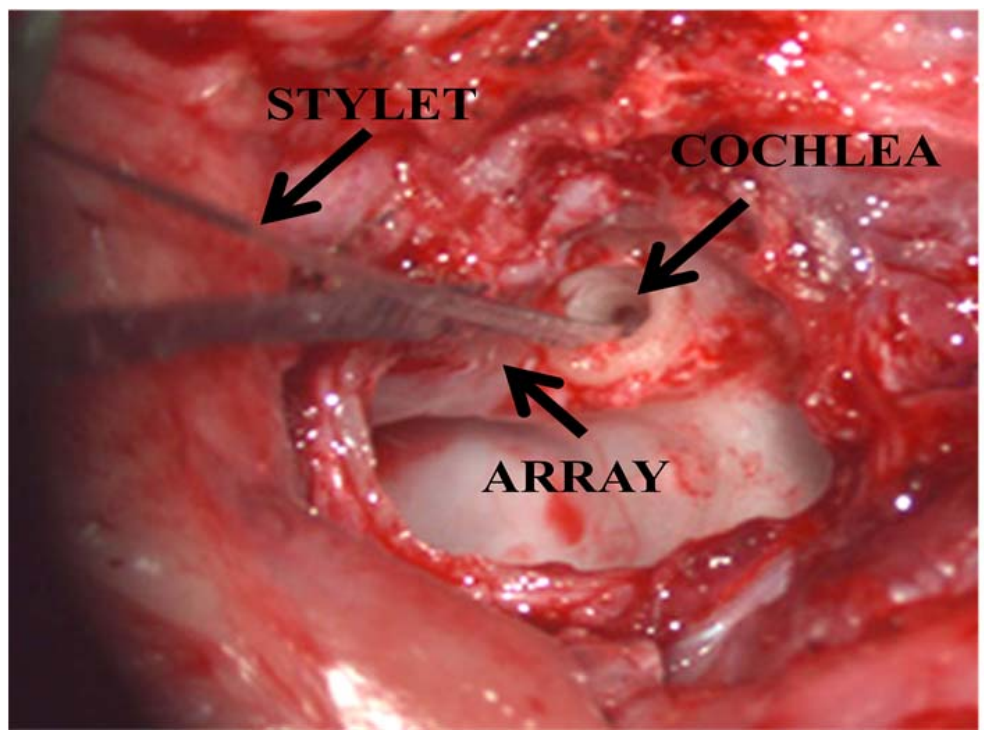

Fig. 3.10: Full insertion of a PET-backed cat array into the cat cochlea.

Although some successful inerstions were achieved, the yield was low and failures usually occurred as a result of delamination between the PET tubes and the parylene array. It was found that the UV adhesives did not provide sufficient adhesion between the two material layers. Alternate biocompatible adhesives were not found to improve adhesion. In addition to the problems with adhesion, attachment of the arrays to the PET backing was a tedious and difficult process with low yield. To address the issues of deformation, adhesion and attachment complexity, Poly Lactic Acid (PLA) stiffeners were developed [6].

Hot embossed PLA films provide stiffness and curl to the arrays, including bonding the PLA incorporated arrays to a detachable PET insertion tool with a biodegradable polymer (Fig. 3.11). 


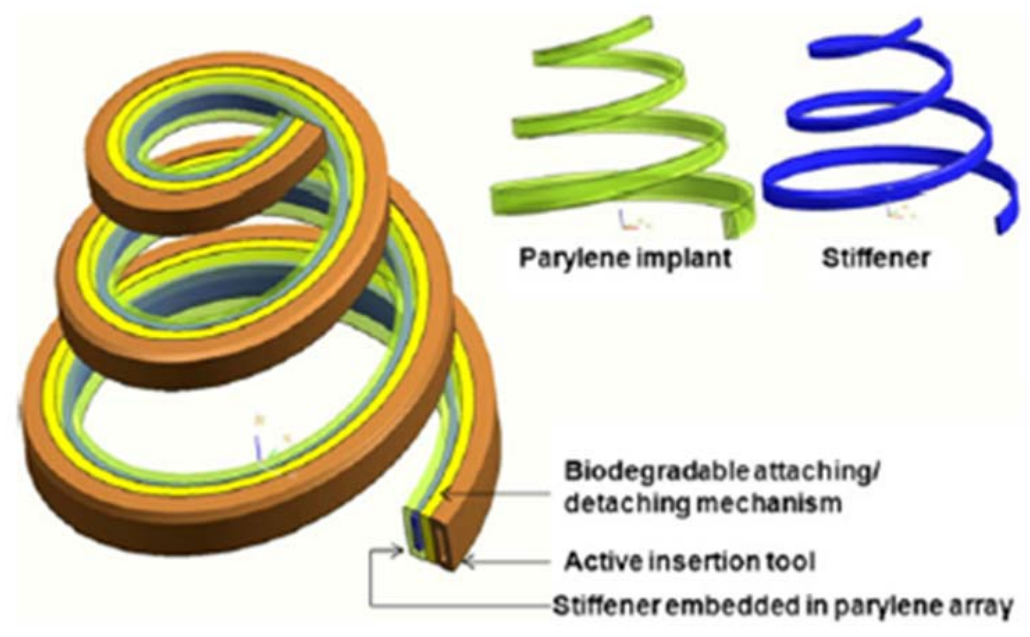

Fig. 3.11: Proposed hybrid Assembly of PLA stiffener, parylene array and PET insertion tool [6].

To reduce the amount of hybrid assembly necessary, the PLA films will be incorporated into the parylene array fabrication process (Fig. 3.12). Fabrication begins with the deposition of parylene over a silicon carrier (Fig. 3.12a). Next, PLA is hot embossed over the parylene into the form of the array (Fig. 3.12b). The PLA field area around the array is etched with oxygen plasma RIE with a gold masking layer (Fig. 3.12c). The array is then built on top of the PLA base using the steps outlined in chapter 2 (Fig. 3.12d-f).

(a)

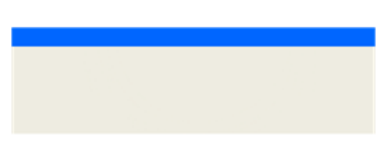

(d)

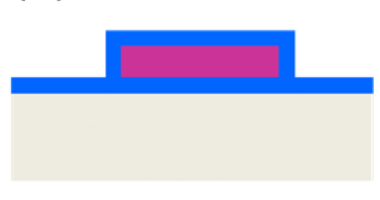

Carrier Substrate

- Tunnel Overlap (b)

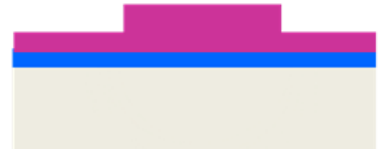

(e)

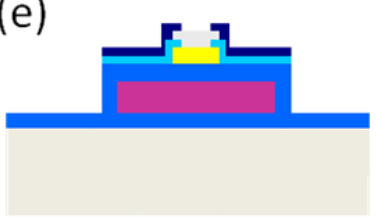

- ParyleneTube Interconnect (c)

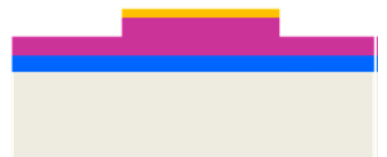

(f)

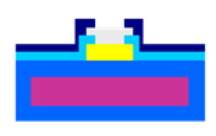

Enclosure Insulation Metal Sites Au Mask

Fig. 3.12: PLA integration into the parylene process 
Hot embossing of the PLA over parylene coated wafers has been successfully achieved (Fig. 3.13), and future work will involve combining the hot embossed PLA with the full parylene array process and attaching the PET insertion tool.

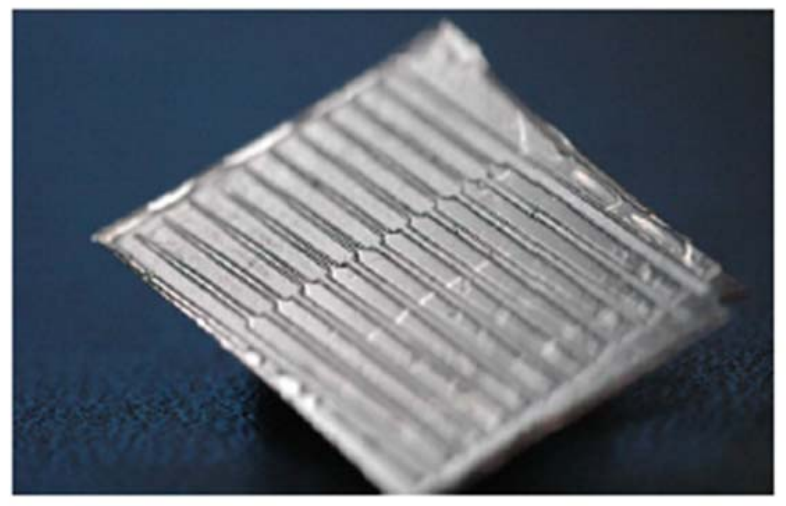

Fig. 3.13: Hot embossed PLA over a parylene substrate [6].

Even with the partially integrated PLA films, a significant amount of post processing would still need to be done in order to include the PET insertion tool using biodegradeable polymers. Towards the goal of eliminating all hand assembly steps in lead design, monolithic backings were investigated. These backings included mechanisms for stiffness and curvature, and, when necessary, as a built-in method of insertion.

\subsubsection{Monolithic Curl Frames}

To place the electrode sites in close proximity to the target cells, curl can be built into a cochlear electrode array so that it hugs the modiolus. The closer the sites are to the nerves of interest, the smaller the threshold level of stimulation will be. Although most commercial implant companies produce straight arrays, the most commonly used technology (Nucleus Contour) is curved. As such, we desired to produce not only a straight thin-film array, but a curved one as well. Although most other cochlear implant leads, thin-film and otherwise, use hybrid additions to produce curvature, the work here is towards monolithically integrated curl structures.

Two stress balancing techniques were used to create curl in the cochlear electrode array. In the first technique, metal layers of opposing stresses are added to the top and bottom of the array in a metal-array-metal (M3) layering. The M3 method is done by 
stress-compensating the bulk of the array (insulation layers and interconnect) to have low curvature, and then adding curvature-inducing bottom and top metal layers. In the second technique, the array interconnect is stress-compensated to have low to zero curvature, and the first and final parylene layer thicknesses are imbalanced to induce stress-controlled curvature in a parylene-interconnect-parylene layering design (PIP). Both techniques were simulated to gain insight into which would induce the greatest curvature. Then, both methods were implemented to compare their individual compatibility with the complete array fabrication process. Considering the high material stresses involved in both, it was anticipated that a trade-off would have to be made between achieving optimum curvature and optimum process yield.

The M3 and PIP techniques were each modeled separately with the goal of gaining intuition into how their layer stresses and thicknesses affect the radius of curvature. As such, a simple beam of three layers was used for each case. The triple layer beam is modeled as two bi-layer beams whose individual curvatures are summed. Curvature $(k)$ of the bilayer beam is a result of the difference in strain $(\square \varepsilon)$ between the bottom and top layers. The equations for determining curvature are [8]:

$$
\begin{aligned}
& \text { (1) } E_{b}=\frac{E_{M b}}{1-v_{M b}^{2}}, E_{t}=\frac{E_{M t}}{1-v_{M t}^{2}}, \\
& \text { (2) } \Delta \varepsilon=\frac{\sigma_{t}}{E_{t}}-\frac{\sigma_{b}}{E_{b}}, \\
& \text { (3) } k=\frac{6 E_{b} E_{t} t_{b} t_{t}\left(t_{b}+t_{t}\right) \Delta \varepsilon}{E_{b}^{2} t_{b}^{4}+E_{t}^{2} t_{t}^{4}+2 E_{b} E_{t} t_{b} t_{t}\left(2 t_{b}^{2}+2 t_{t}^{2}+3 t_{b} t_{t}\right)},
\end{aligned}
$$

where $\sigma_{t, b}$ represents stress, $t_{t, b}$ represents thickness, and $E_{M t, M b}$ represents the modified Young's modulus accounting for Poisson's ratio $v_{M t, M b}$. The radius of curvature $(r)$ is simply:

$$
\text { (4) } r=\frac{1}{k}
$$

The above equations were used to model an approximation of the expected curvature based off of the ratio of layer thicknesses $\left(t_{b} / t_{t}\right)$, and the layer stresses $\sigma_{t} / \sigma_{b}$. The model used an $11 \mathrm{~mm}$-long beam. 
It is necessary that the arrays curl in the positive direction (out-of-plane) once released from the substrate. To get this direction of curvature using the M3 technique, the top and bottom stresses need to be tensile and compressive, respectively. For the compressive material, Ti-Ir was chosen since Ir can be deposited at high stresses of greater than -2GPa. For the tensile layer, $\mathrm{Cr}-\mathrm{Au}$ and $\mathrm{Ti}-\mathrm{Pt}$ bottom layers were considered. In the model, only Ir and Pt are considered since the Ti adhesion layer is relatively thin and has a small stress in relation to Ir and Pt.

Initially $\mathrm{Cr}-\mathrm{Au}$ was considered as a tensile layer because of Au's low Young's modulus of $79 \mathrm{GPa}$. To determine the maximum stress that could be achieved in the $\mathrm{Au}$ layer, the material was deposited at various thicknesses using an Enerjet evaporator. The maximum tensile stress of $112 \mathrm{MPa}$ was achieved with a Cr-Au layer thickness of $200 \AA$ $5000 \AA$. The estimated radius of curvature for the $200 \AA-5000 \AA$ layer thickness over a $200 \AA-1700 \AA$ layer of Ti-Ir was $171 \mu \mathrm{m}$.

Ti-Pt was investigated as an alternative to $\mathrm{Cr}-\mathrm{Au}$, since Ti-Pt can achieve higher stresses at thinner deposition layers. However, the Young's modulus of Pt is over twice that of $\mathrm{Au}$, so there is a trade-off in stiffness for the higher stress value. Despite the increased stiffness, due to its high tensile stress, the model suggests a $75 \mu \mathrm{m}$ radius of curvature can be achieved for a $200 \AA$ - $1000 \AA$ layer of Ti-Pt over a $200 \AA-1700 \AA$ layer of Ti-Ir. For this reason, Ti-Pt was chosen as the tensile material in the M3 design.

The PIP design uses the strain in the two parylene layers to produce curl. In contrast to the M3 technique, there is less than a few megapascals difference between the stresses in the top and bottom parylene layers, and the stress itself is quite low. For thin depositions of parylene (less than $3 \mu \mathrm{m}$ ), the measured stress was approximately $-4 \mathrm{MPa}$. As the layer thickness increased, the stress became more tensile, with an $11 \mu \mathrm{m}$ layer of parylene having $0.3 \mathrm{MPa}$ of stress. Despite the low stress of the parylene-C layers, the layer thicknesses are such that a significant amount of curvature can be developed in the arrays. To better understand what effect the top and bottom parylene thicknesses have on the curvature, the thickness of each layer was increased or decreased with respect to the other and the model was used to observe the change in stress with the ratio $\left(P_{t} / P_{b}\right)$, where $P_{b}$ is the bottom layer of parylene and $P_{t}$ is the top. For a set beam height $h$, it can be seen that for ratios greater than 1 , the curvature is positive, and for ratios less than 1 , 
the ratio is negative (Fig. 3.14, Fig. 3.15). Therefore, the array will curve upwards when the top layer is thicker than the bottom and curve downwards for the opposite case (Fig. 3.16, Fig. 3.17). It can be seen that the radius of curvature decreases for higher values of $P_{t}$ or $P_{b}$; however, increasing the thickness also increases the beam height, which has a negative effect on the curvature; as such, the curvature plateaus for thicknesses greater than $10 \mu \mathrm{m}$ with a $P_{b} / P_{t}$ ratio of 5 . The modeled minimum radius of curvature that can be achieved with parylene depositions of at least $2 \mu \mathrm{m}$ is $1,583 \mu \mathrm{m}$.

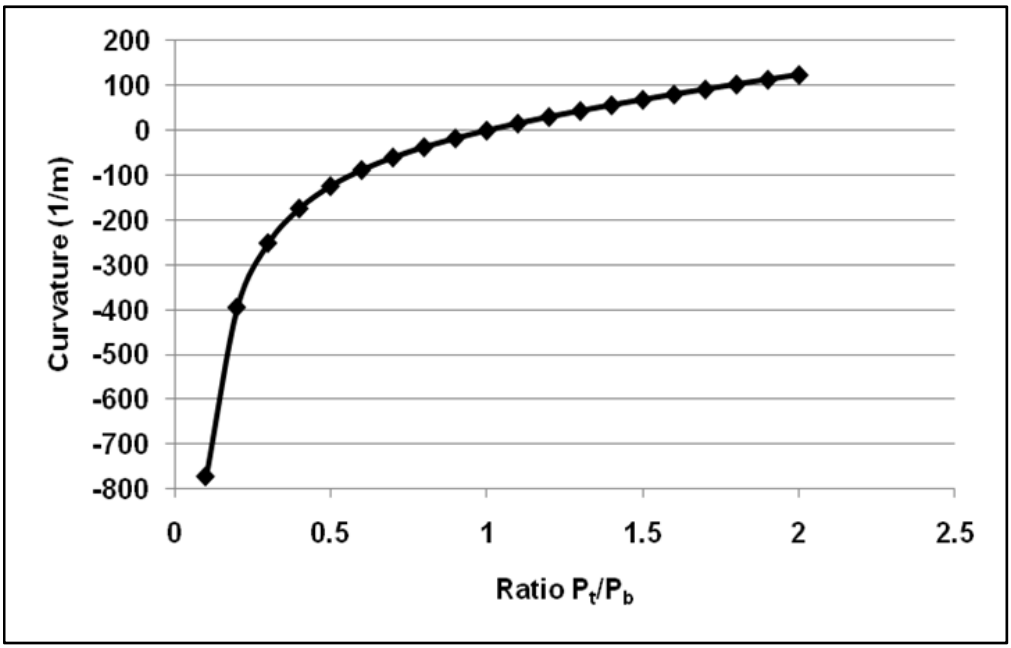

Fig. 3.14: Simulated beam curvature versus the ratio of top and bottom parylene layer thicknesses. The overall height of the stacked layer is $20 \mu \mathrm{m}$.

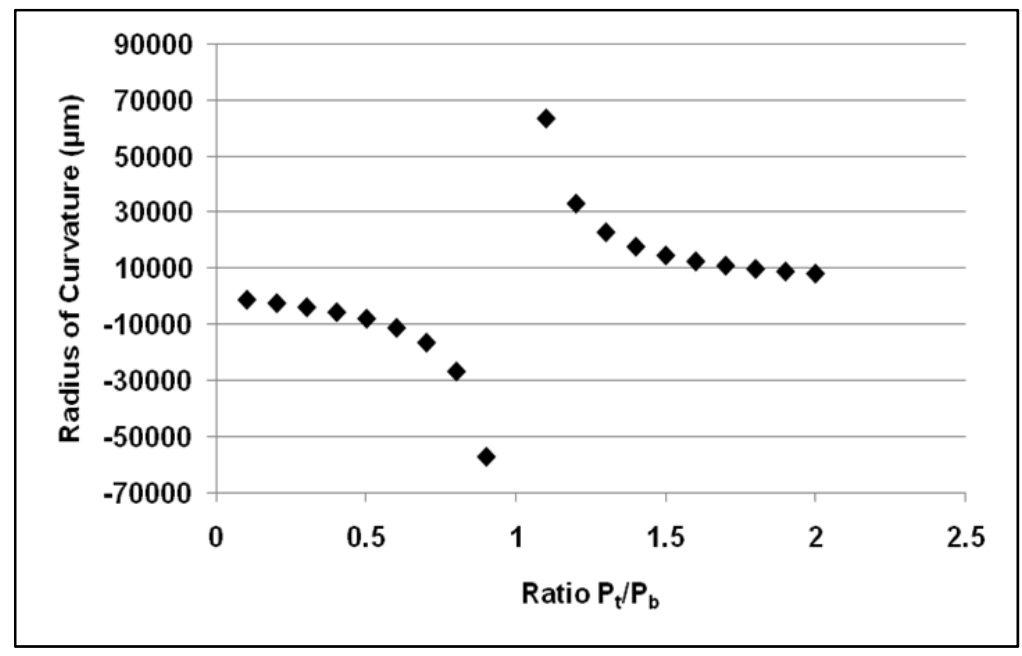

Fig. 3.15: Simulated radius of curvature versus the ratio of top and bottom parylene layer thicknesses. The overall height of the stacked layer is $20 \mu \mathrm{m}$. 


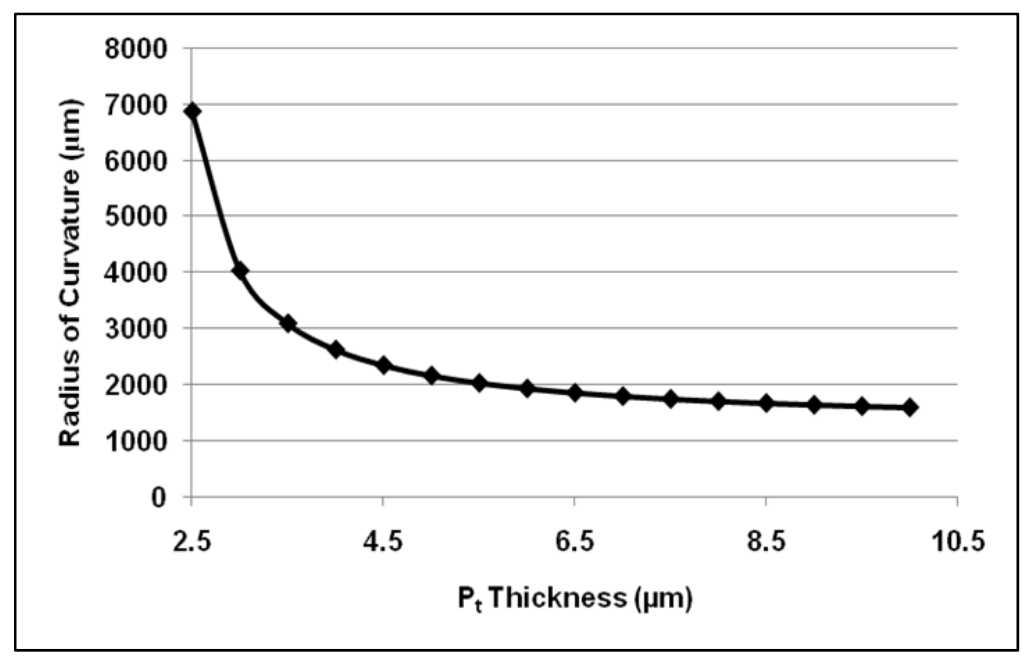

Fig. 3.16: Simulated beam curvature for increasing thickness of the top parylene layer over a $2 \mu \mathrm{m}$ thick bottom layer.

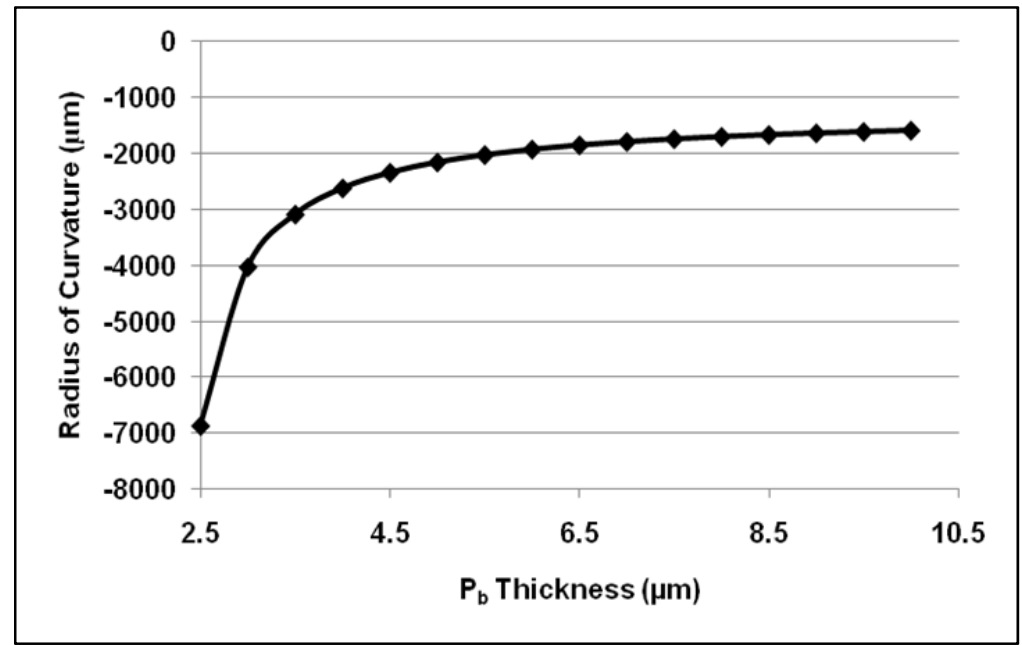

Fig. 3.17: Simulated beam curvature for increasing thickness of the bottom parylene layer under a $2 \mu \mathrm{m}$ thick top layer.

The radius of curvature of the first turn in the cat and guinea pig cochlea is $1.5-1$ $\mathrm{mm}$. Therefore, it is desirable for the array to achieve a radius of curvature of less than 1 $\mathrm{mm}$. However, the smaller the radius is below this value the more closely it will hug the modiolus wall, and the frame will be designed to achieve a radius of curvature that is as small as possible. In comparison to the PIP technique, the M3 method has the potential to achieve a much smaller radius of curvature. As such, fabrication began with the development of the M3 process. As discussed below, processing of the M3 design 
resulted in poor yields, and ultimately the PIP technique was adopted, with slight modifications.

The M3 curl frames were incorporated into the array process using two additional metal deposition steps (Fig. 3.18). Following vapor deposition of the parylene substrate layer, high stress Ir is deposited as a base layer (Fig. 3.18a-b); next the parylene array is built up as discussed in chapter 2 (Fig. 3.18c). Before depositing the final substrate layer of parylene, Ti-Pt is evaporated over the arrays in all areas excluding the Ti-Ir sites (Fig. $3.18 d-f)$.

(a)

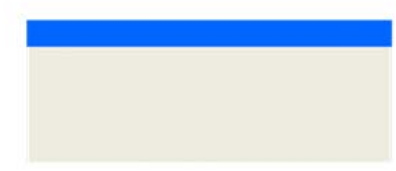

(d)

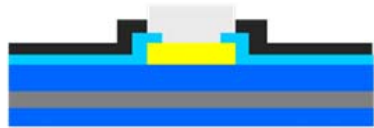

Carrier Substrate

Tunnel Overlap (b)

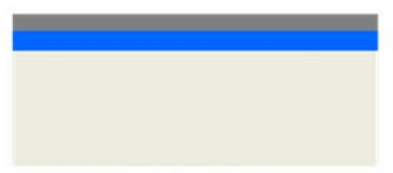

(e)

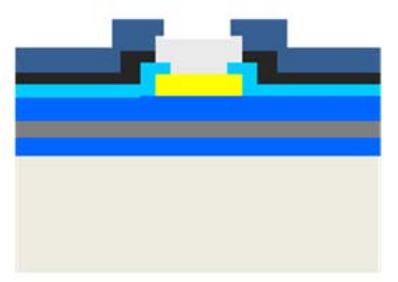

(c)

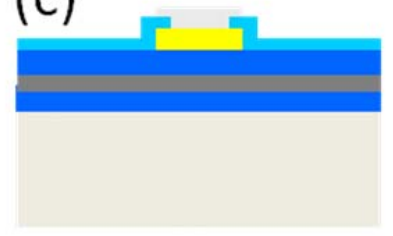

(f)

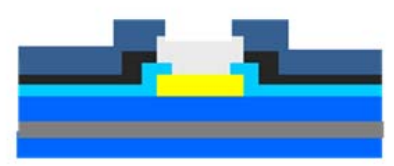

Fig. 3.18: The M3 fabrication process.

A number of arrays were fabricated in this way (Fig. 3.19). The devices achieved curl in the desired direction upon release from the carrier wafer. However, the radius of curvature was not as high as expected from the analytical analysis. In part, this was due to the addition of parylene and Ti-Ir (sites) layers, which were not accounted for in the model. However, the high stress Ti-Ir was prone to delaminate from the parylene substrate, and this not only diminished or eliminated the curvature, but also damaged the mechanical robustness of the arrays. Device yields were quite low and the curvature was not stable enough to withstand the subsequent addition of monolithic positioners. 


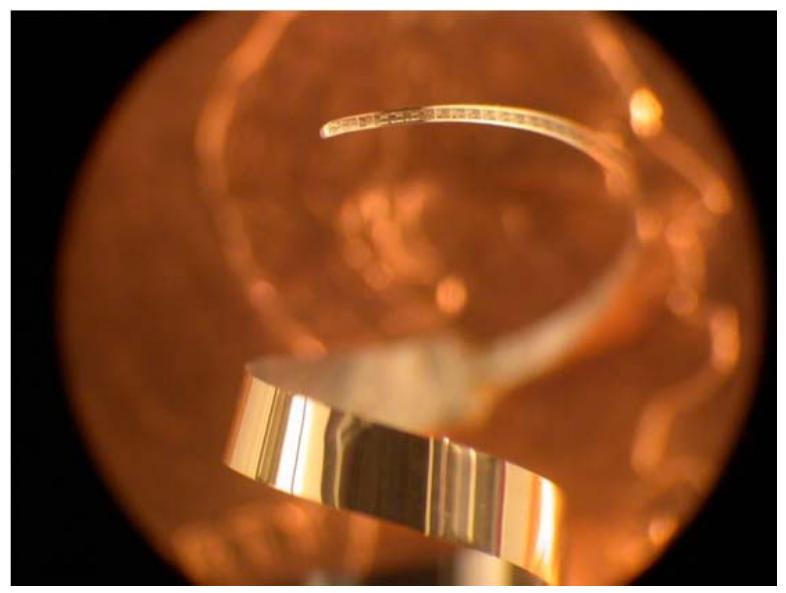

Fig. 3.19: Curled GP array with high stress Ti-Ir and Ti-Pt layers.

As an alternative to M3, the PIP designs were implemented through fabrication since this technique did not require any additional high-stress material layers or array process modifications. Guinea pig arrays were produced with $2 \mu \mathrm{m}$-thick bottom layers and $8 \mu \mathrm{m}$-thick top layers of parylene (Fig. 3.20). The devices curled in the desired direction following release from the wafers, but as expected the curvature was not as high as desired. To increase the curvature the arrays were heated on a hotplate set to $120^{\circ} \mathrm{C}$. It has been shown that when parylene-C is heated to high temperatures (up to $250{ }^{\circ} \mathrm{C}$ ), the residual stress increases by a factor greater than 6 [7]. This increased residual stress greatly enhances the curvature of the devices (Fig. 3.21). The devices could be fabricated with $80 \%$ yield and achieved curvature radii of less than $0.5 \mathrm{~mm}$. To enable the arrays to be surgical placed inside the cochlea, it is necessary to straighten them first. Monolithic positioners were designed to place the arrays using the Advanced-Off-Stylet (AOS) method. The following sections discuss this in greater detail.

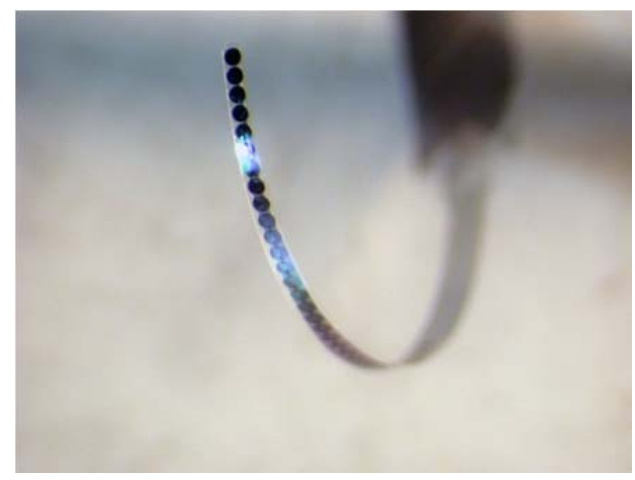

Fig. 3.20: Pre-curved guinea pig array as released from wafer. 


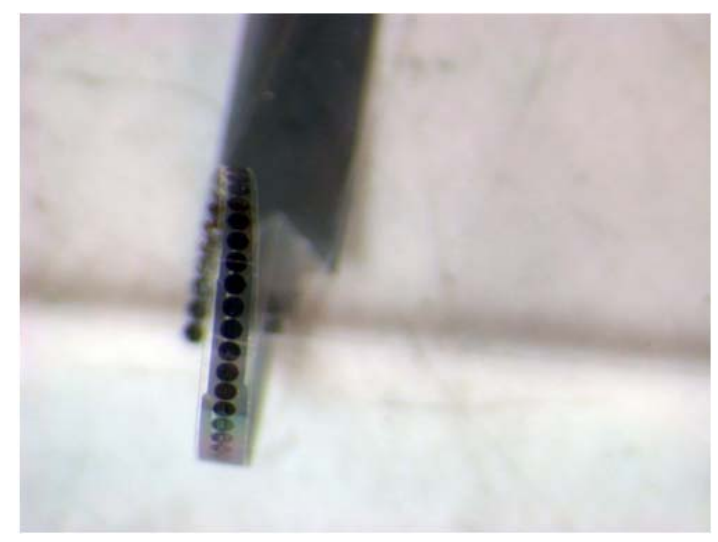

Fig. 3.21: Heated treated guinea pig array.

\subsubsection{Monolithic Positioner-Stiffeners}

The ultra-flexible flat parylene arrays described in chapter 2 cannot be inserted into the cochlea without increasing their stiffness and/or curling them. With an appropriate amount of stiffness, the arrays could be directly positioned inside the cochlea with tweezers or a plunger apparatus. The stiffness has to be such that the array can withstand collisions with the back wall of the scala tympani. In all straight-array designs, collision is inevitable and is a normal part of insertion. Back-wall contact forces of $25 \mu \mathrm{N}$ have been recorded in cochlear implant insertions [8]. If curl is added to the device, with proper insertion it could glide along the inner wall of the modiolus, having minimal contact with the back wall, and the stiffness could be reduced. For the cochlear arrays of this work, both straight and curled devices were designed. For the straight arrays, monolithic channels were integrated into the arrays to create a stiffness suitable for direct insertion. In the case of the curled arrays, a variation of these same channels were integrated to so that the Advanced-off-Stylet insertion method could be used. Since the parylene arrays are pre-curled for modiolus hugging, before positioning them inside the cochlea it is necessary to straighten them out. This can be accomplished using the AOS insertion technique, which results in lower insertion forces and array stiffness requirements [8].

Two types of channels were investigated (Fig. 3.20, Fig. 3.21). The first was a uniform, continuous channel with slots etched out of it to create parylene rings separated 
by open space gaps; the second was a non-uniformly slotted channel where the gaps decreased in size going from the tip to the base of the array.

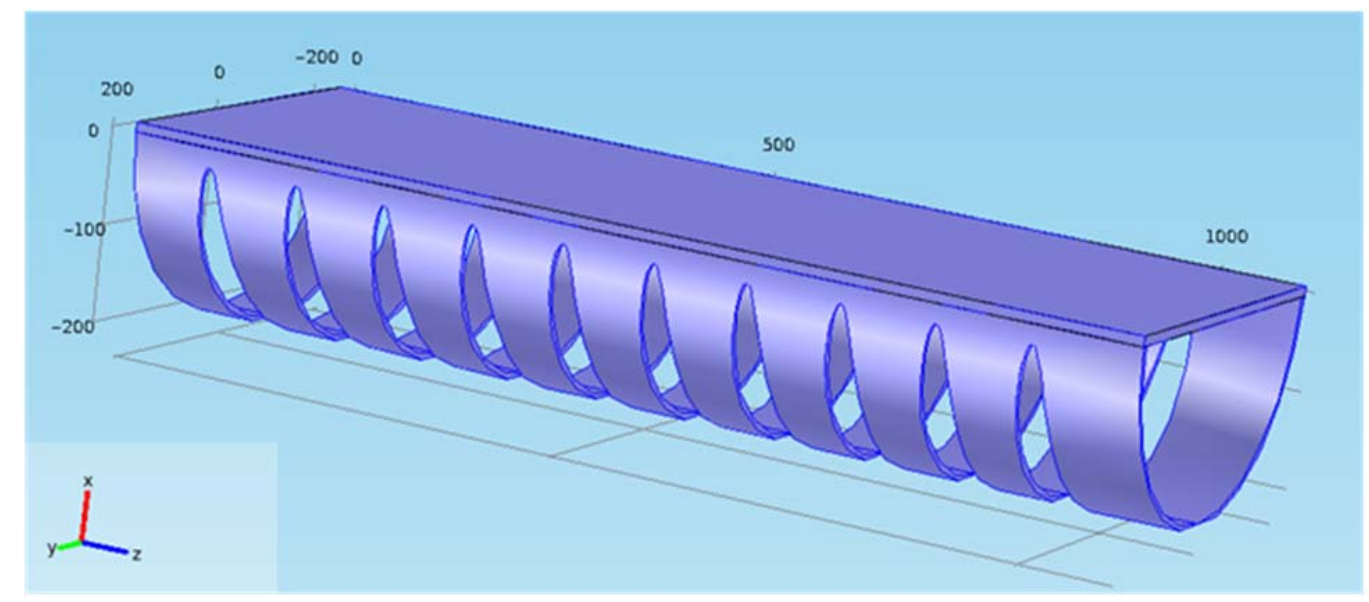

Fig. 3.20: Uniformly spaced rings on a slotted channel.

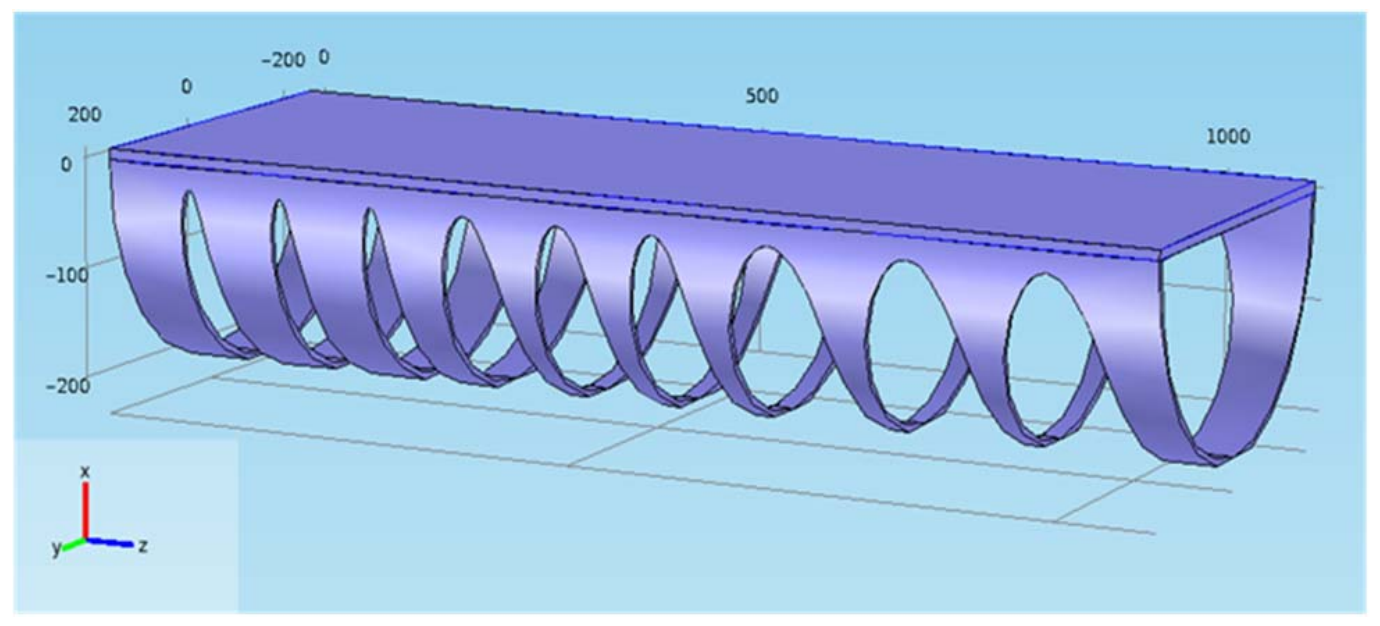

Fig. 3.21: Non-uniformly spaced rings on a slotted channel. Gap length increases along the array.

A uniformly slotted channel was designed and modeled with FEA (Fig. 3.22). The model was used to determine what maximum stiffness could be achieved with the slotted structure and how the ring length $\left(\mathrm{R}_{\mathrm{L}}\right)$ and gap length $\left(\mathrm{G}_{\mathrm{L}}\right)$ can be varied to set values within the range.

FEA was conducted using the COMSOL Multiphysics software package to model changes in the ringed channel stiffness in relation to changes in $R_{L}$ and $G_{L}$. A 3D structural mechanics MEMS module was used. In the model, the channel is represented 
by a $1.1 \mathrm{~mm}$-long, $400 \mu \mathrm{m}$-wide beam with a $400 \mu \mathrm{m}$-wide channel beneath it. The thickness of the channel wall was $5 \mu \mathrm{m}$. An isotropic material model of parylene was used having a Young's modulus of 4GPa, Poisson's ratio of 0.4 , and density of 1289 $\mathrm{kg} / \mathrm{m}^{3}$. The beam was fixed on one end and a perpendicular force of $125 \mathrm{~N} / \mathrm{m}^{2}$ was distributed over the beam's bottom surface area in the x-direction, deflecting it upwards (Fig. 3.30). The stiffness $(k)$ of the channel was calculated from its deflection $(\delta)$ under the applied force $(\mathrm{F})$ :

(1) $\quad k=\frac{F}{\delta}$

To approximate the stiffness of the channel for the full $11 \mathrm{~mm}$ length of the array, standard mechanics equations were used for a beam fixed on one end [11]:

(2) $\delta=\frac{F L^{3}}{3 E I}$

For constant Young's modulus (E), moment of inertia (I), and applied force (F), $k$ is:

(3) $\quad k=\frac{3 E I}{L^{3}}$

For channel lengths $L^{\prime}$ longer than $1 \mathrm{~mm}$, the stiffness $k^{\prime}$ can be approximated by:

(4) $k^{\prime}=k\left(\frac{L^{\prime}}{200 \mu m}\right)^{3}$ 
Surface: Total displacement $(\mu \mathrm{m})$

$\Delta 11.49$

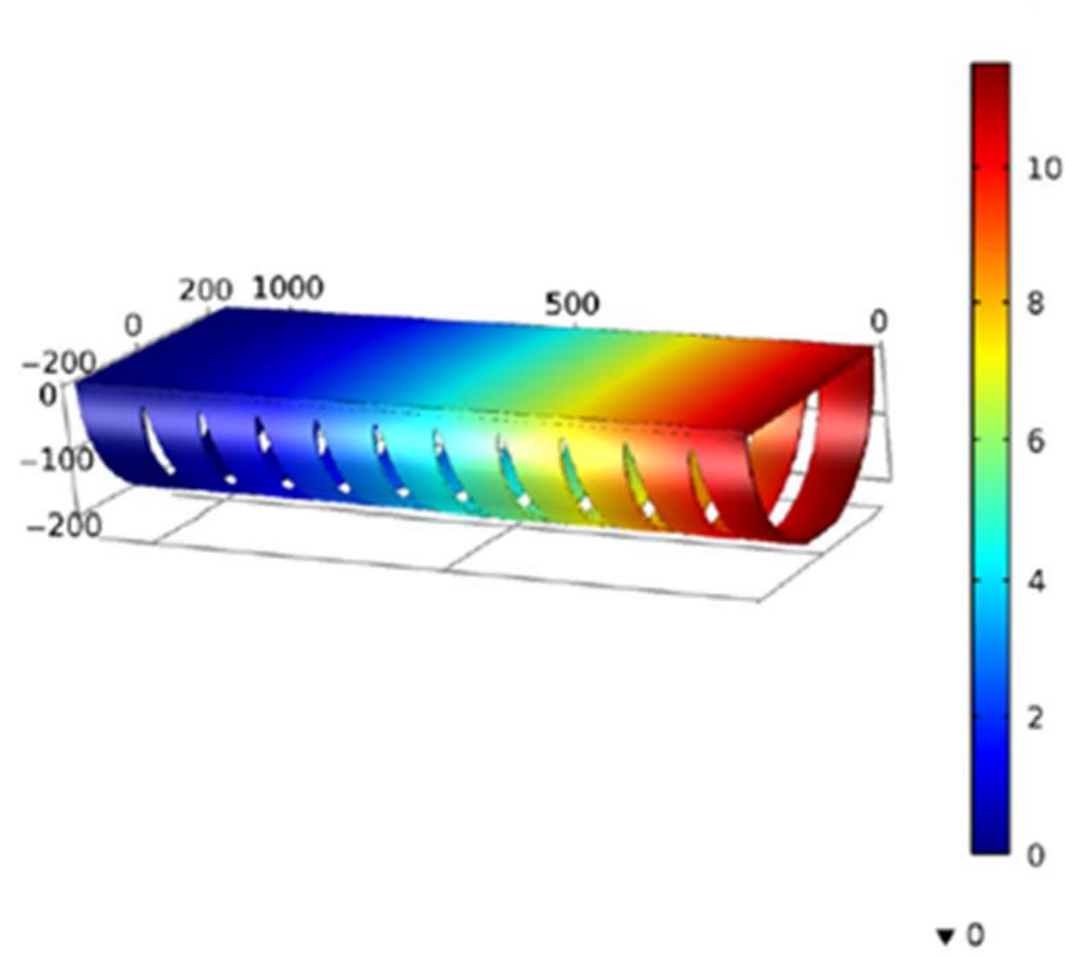

Fig. 3.22: The 3D FEA model of a ringed channel.

Several simulations were done, changing the ratios, values, and percentages of areas covered by $R_{L}$ and $G_{L}$. The goal was to determine how changing the parameters $R_{L}$ and $\mathrm{G}_{\mathrm{L}}$ changes the stiffness of the channel. Of those simulations, two types gave the best indication as to how stiffness varies with changes in $\mathrm{R}_{\mathrm{L}}$ and $\mathrm{G}_{\mathrm{L}}$. In one variation, the pitch covered by $R_{L}$ and $G_{L}$ was kept constant and $G_{L}$ was increased. In another, the ratio of $R_{L} / G_{L}$ was set to one and both $R_{L}$ and $G_{L}$ were increased.

It can be seen that the stiffness of the channel decreases as $\mathrm{G}_{\mathrm{L}}$ increases for situations in which the pitch is kept constant (Fig. 3.23, Fig. 3.24). For this case, the percentage of total area covered by gaps goes up and thus there are less fixed regions and greater flexibility. 


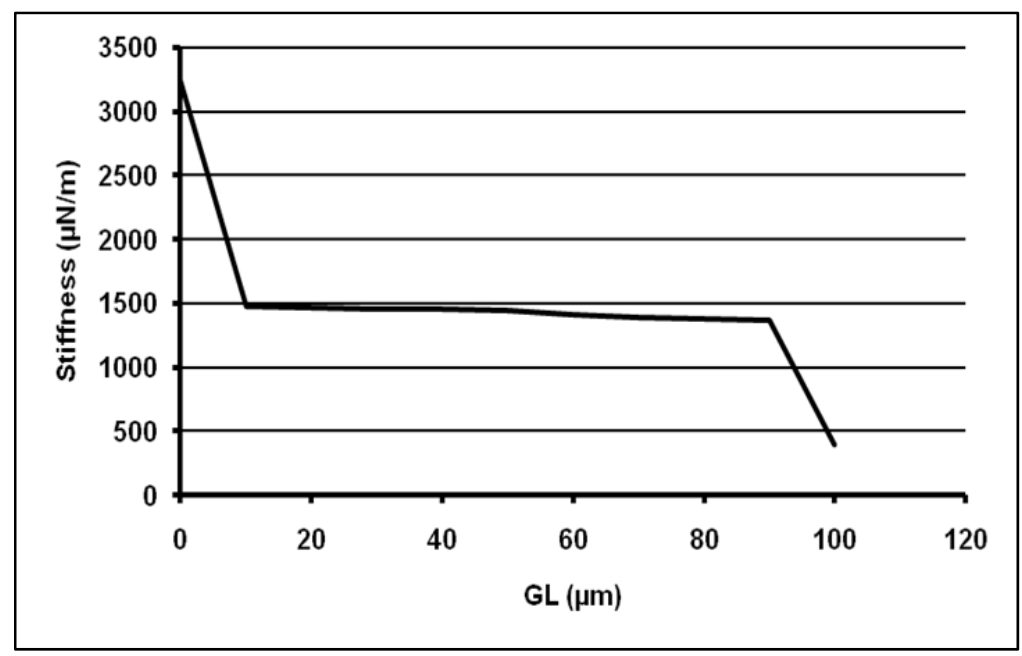

Fig. 3.23: Stiffness of the ringed-channel design versus the ring gap length for a constant ring pitch of a $100 \mu \mathrm{m}$.

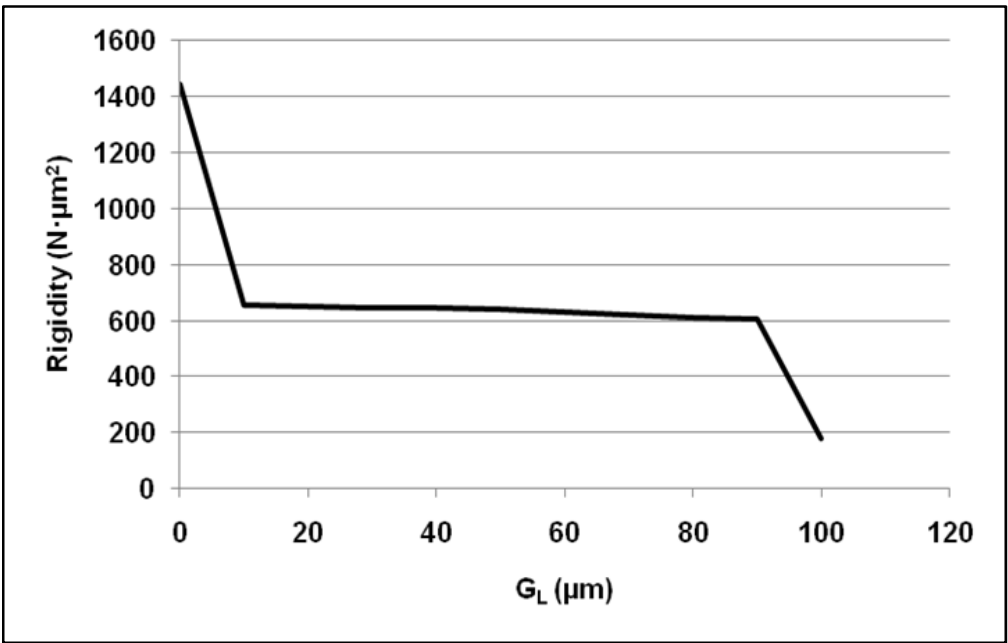

Fig. 3.24: Rigidity of the ringed-channel design versus the ring gap length for a constant ring pitch of a $100 \mu \mathrm{m}$.

When the ratio $R_{L} / G_{L}$ is kept constant at one and $R_{L}$ is increased, the stiffness of the channel decreases for increasing lengths of $\mathrm{G}_{\mathrm{L}}$ (Fig. 3.25, Fig. 3.26). The percentage of area covered by the rings and gaps is kept constant in this variation at $50 \%$ each. Although $G_{L}$ is being increased and this has a diminishing effect on the stiffness, $R_{L}$ is being increased as well and the stiffness of the channel is more sensitive to increases in ring length. This results in an overall decrease in stiffness. 


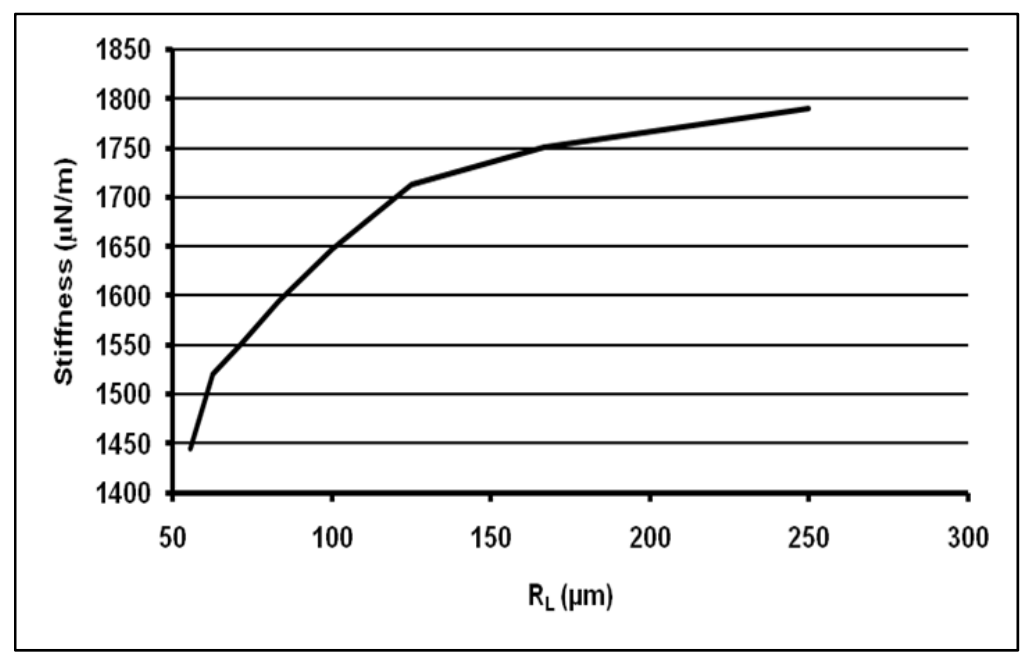

Fig. 3.25: Stiffness versus ring length for a constant $R_{L} / G_{L}$ ratio of 1 .

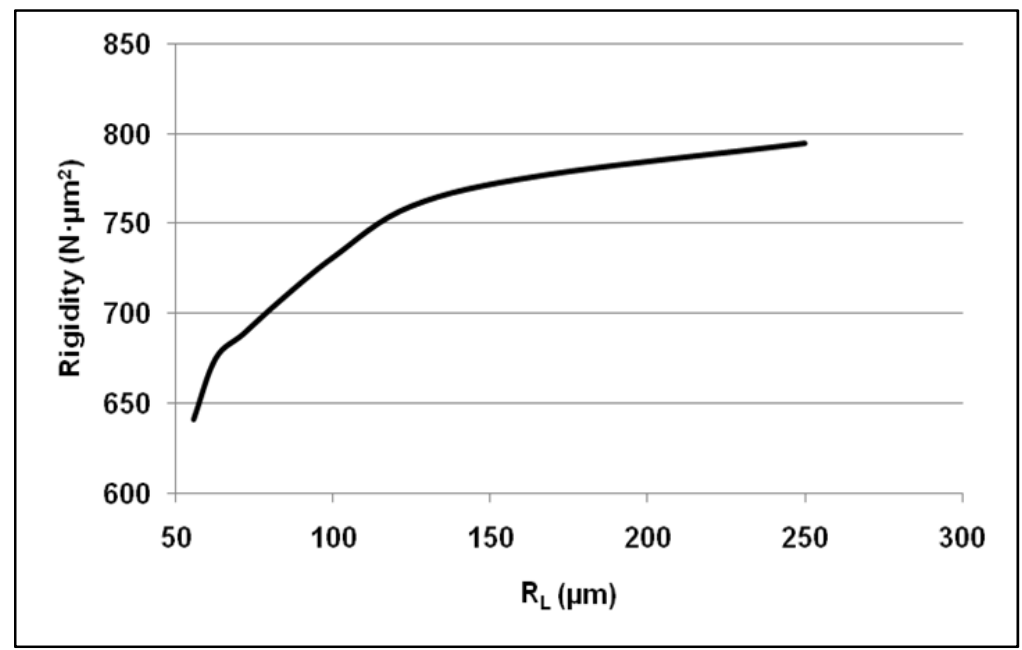

Fig. 3.26: Rigidity versus ring length for a constant $R_{L} / G_{L}$ ratio of 1 .

The maximum rigidity of the channel, $1.4 \mathrm{kN} \cdot \mu \mathrm{m}^{2}$, occurs for the case where there are no gaps. The minimum of approximately $0.2 \mathrm{kN} \cdot \mu \mathrm{m}^{2}$, occurs when there are no rings. As such, the model estimates that a continuous channel addition to the parylene arrays can increase the stiffness to that of a planar array by $700 \%$. In the past, flattened PET tubing of stiffness $2.3 \mathrm{kN} \cdot \mu \mathrm{m}^{2}$ [6] achieved successful insertions into GP and cat cochlea molds. The $1.4 \mathrm{kN} \cdot \mu \mathrm{m}^{2}$ stiffness of the parylene rings is $40 \%$ less than this, but techniques that included grading the stiffness of the channel, as discussed later on, increased the ability of the flexible arrays to achieve insertion depths comparable to the PET tubes, and with less trauma. 

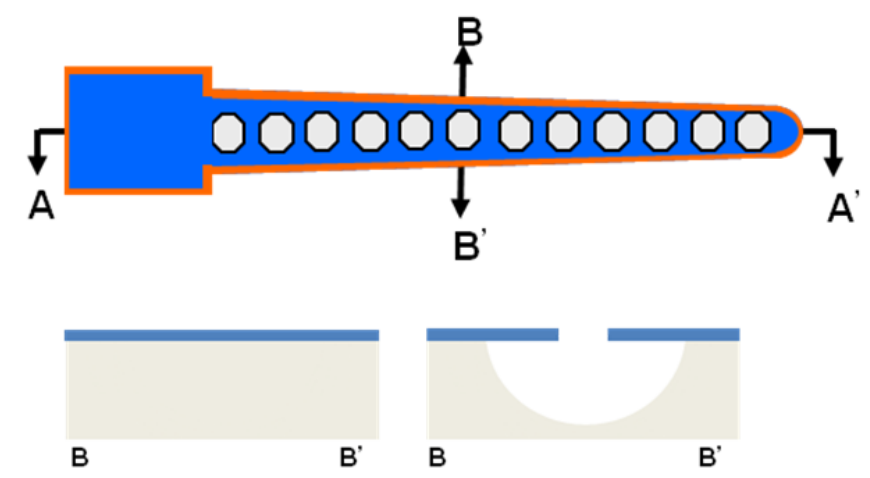

(a)

(b)

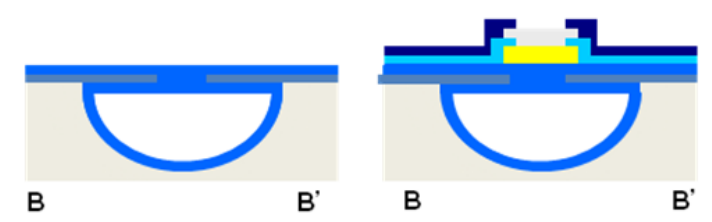

(c)

(d)

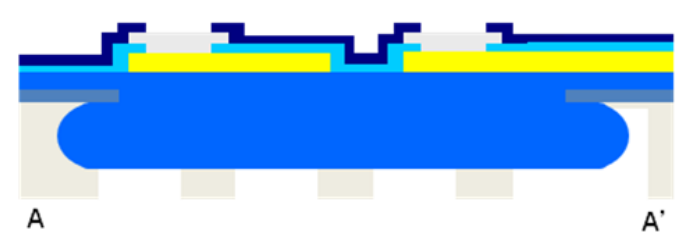

(e)

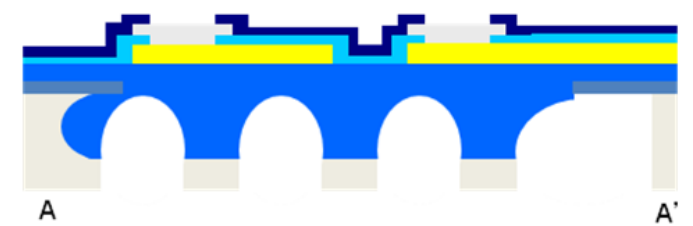

(f)

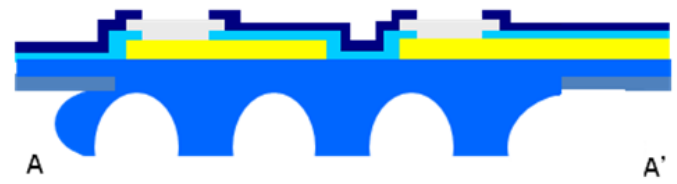

(g)

$\begin{array}{ll}\text { Carrier Substrate } & \text { ParyleneTube } \mathbf{~ E n c l o s u r e ~} \\ \square \text { Tunnel Overlap } & \text { Curl Strips } \\ \text { Insulation } & \text { Metal Sites }\end{array}$

Fig. 3.27: Process flow for the fabrication of ringed-channel devices. 
The ringed channels were fabricated under the array with several modifications to previous buried-channel processes $[9,10]$. Fabrication begins with the vapor deposition of a thin $1 \mu \mathrm{m}-2 \mu \mathrm{m}$ masking layer of parylene (Fig. 3.27a). A rectangle $5 \mu \mathrm{m}$ wide and $16 \mathrm{~mm}$ long is etched out of the parylene using a directional $\mathrm{O}_{2}$ plasma. The rectangular opening is isotropically undercut by $100 \mu \mathrm{m}-150 \mu \mathrm{m}$ to form a channel using a $\mathrm{XeF}_{2}$ etch (Fig. 3.27b). The channel walls are then coated with parylene and the overlap opening is sealed off (Fig. 3.27c). Due to the small width of the parylene opening, the channel can be completely sealed by depositing a $7 \mu \mathrm{m}$ layer of parylene. Once the channel is filled in, the parylene stimulating array is built on top of it (Fig. 3.27d). Then the backside of the silicon wafer is selectively masked and etched using DRIE (Fig. 3.27e). The exposed regions of the tunnel are then removed in a directional $\mathrm{O}_{2}$ plasma etch (Fig. 3.27f). Lastly, the entire array is released from the carrier wafer using a $2 \%$ $\mathrm{KOH}$ solution (Fig. 3.27g).

In the above process, a dry isotropic etch is performed as opposed to a wet etch to keep the overlap opening from expanding after undercutting. Any type of wet processing on the wafer in-between the undercut (Fig. 3.27c) and fill-in steps (Fig. 3.27d) results in an offset between the left and right overlaps of the tunnel (Fig. 3.28). These offsets can be tens of microns and prevent complete fill-in of the channel.

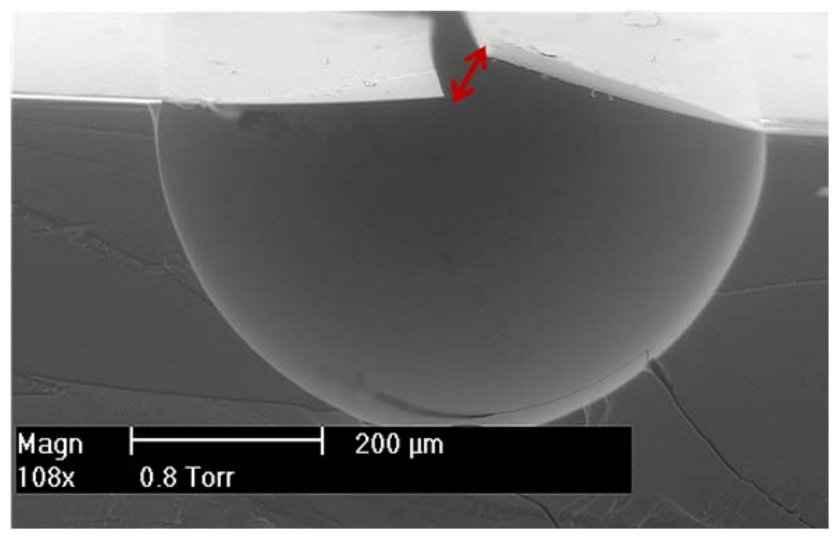

Fig. 3.28: Overlap of channel offset as a result of wet processing.

With a $\mathrm{XeF}_{2}$ etch, as opposed to an RIE, the etch selectivity between parylene and silicon is very high, and so the parylene can act as a mask for the undercut, eliminating the need for photo resist masks which would require wet removal in-between the 
undercutting and fill-in steps. In building the tunnel under the array, care had to be taken to properly seal off the overlap opening. If there were any minor cracks in the opening, then disconnections would occur in the $\mathrm{Cr} / \mathrm{Au}$ lead lines built on top of the channel. Additionally, it was observed that photoresist solvents could get trapped in the tunnel. Once in the channel, these solvents would expand whenever the process wafers were placed on a hotplate and cause the substrate parylene layers to deform or burst.

Careful consideration has to be given to ensure that the parylene channel rings and the substrate of the parylene stimulating arrays do not get over etched in the $\mathrm{O}_{2}$ plasma before the exposed regions can be removed. The $\mathrm{O}_{2}$ plasma etch is directed vertically with an applied bias to the platen for accelerating ions to the surface. Despite this bias, however, some lateral etching can still occur [11]. If the etch timing is too long, this lateral etching can undercut the silicon mask and remove the rings (Fig. 3.29). The exposed regions of the channel are curved, so the directional $\mathrm{O}_{2}$ plasma does not etch all surfaces at the same speed. The top of the channel is going to etch the fastest since it is oriented vertically to the etch; however, the sides of the channel are at an angle from the vertical $\mathrm{O}_{2}$ plasma and will etch at a slower rate. The channel wall thickness is $3 \mu \mathrm{m}-5$ $\mu \mathrm{m}$, and the etch rate of parylene in the Plasmatherm 790 RIE system is approximately $0.16 \mu \mathrm{m} /$ minute. Thus, for a $5 \mu \mathrm{m}$ wall thickness the time to etch the top surface of the channel is 32 minutes.

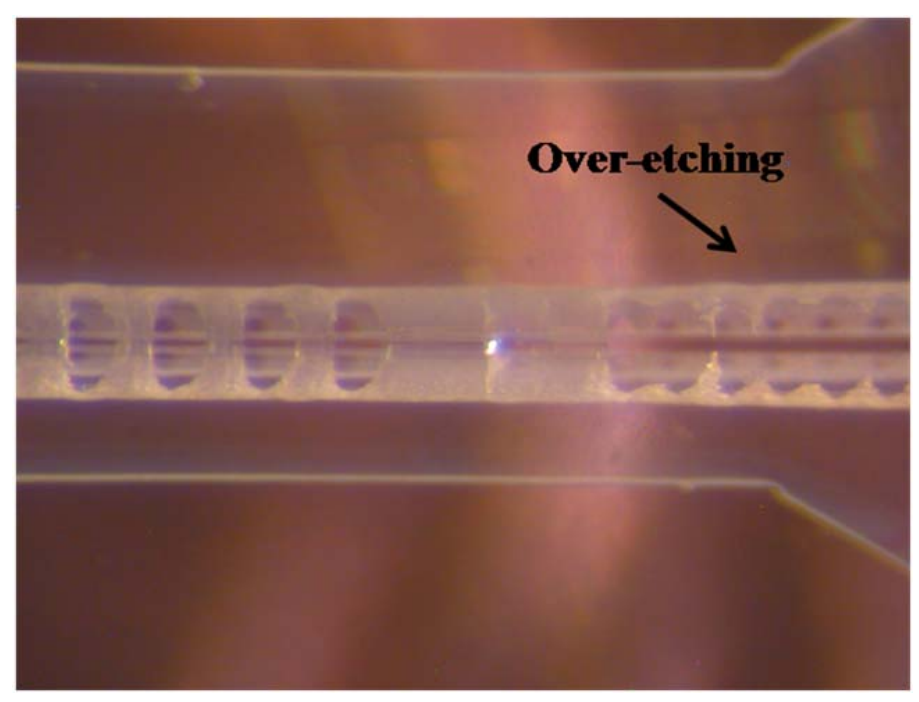

Fig. 3.29: A parylene channel with rings that have been over etched in the $\mathrm{O}_{2}$ plasma. 
It takes approximately one hour to etch away the exposed walls of a $250 \mu \mathrm{m}$ diameter channel to the flat surface of the array. Undercutting can be reduced by adjusting the chamber pressure, RF power, and substrate bias during the etch to increase directionality [11]. However, as the etch becomes more vertical and the lateral etch rate decreases, the time to etch the non-vertical channel walls increases and thus the total etch time will increase. We are currently optimizing the lateral and channel wall etch rates to minimize the residual height of the channel walls.

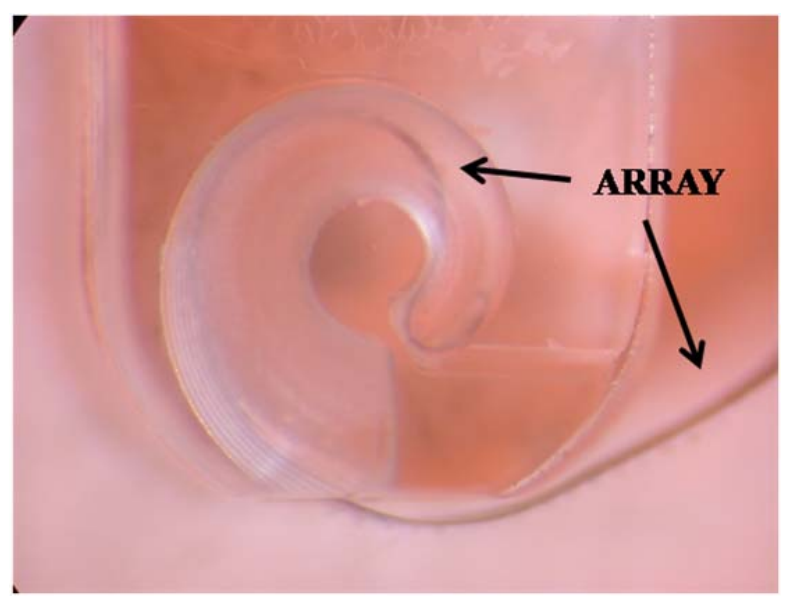

Fig. 3.30: A ringed-channel array inserted into a guinea pig cochlea mold. The channel achieves the desired uniform bending profile.

The arrays have a high degree of stiffness, yet bent conformably to follow the curvature of the guinea pig cochlea mold during insertion (Fig. 3.30). The ringed arrays could be advanced fairly easily into the in-vitro molds when gripped right at the opening. However, in in-vivo guinea pig implants the surgical opening to the cochlea (cochleostomy) is narrow and the closest the arrays can be gripped is at a distance $5 \mathrm{~mm}$ from the ST entrance. At such a distance, the array is likely to buckle on itself instead of advancing into the cochlea. When an array has uniform stiffness and an insertion tool (forceps, tweezers, etc.) is used to advance it into the cochlea, upon hitting the back wall of the cochlea, it will buckle at the region that is midway between the grip location of the insertion tool and the tip of the array. In most cases, buckling will occur at a point outside of the cochlea and make further insertion of the array difficult or impossible. Greater control can be had over where the array bends upon making contact with the back 
wall if the array is divided into segments, where each segment increases in stiffness, such that the apex is less stiff than the base.

Straight GP arrays were developed using this technique. The array was divided into five $2 \mathrm{~mm}$ segments. The width of the rings in each segment was kept constant at 50 $\mu \mathrm{m}$, but the gap length was successively cut by half. The overall effect was a decrease in the number of rings for each segment and an increase in the gap length separating rings. The apical-most segment had the greatest gap length $(1600 \mu \mathrm{m})$ and the basilar segment had the least $(100 \mu \mathrm{m})$. The increment length of $2 \mathrm{~mm}$ was chosen because approximately $2 \mathrm{~mm}$ of a guinea pig straight array can be inserted into a guinea pig cochlea before making contact with the back wall. The arrays were fabricated and tested in-vitro with the use of a GP cochlea mold. Full insertion of the devices was achieved while holding the array at a point $5 \mathrm{~mm}$ from the artificial cochleostomy. In-vivo results using the devices are discussed in chapter 4.

To enable AOS insertion of the pre-curled arrays, the channels integrated onto the backside of the parylene arrays are designed to be used with a passive or active stylet. An active stylet could have thermally-, electrically- or pneumatically-controlled MEMS actuators positioned down the stylet for articulated insertion. Micro-wires can be used as passive stylets, where curling and uncurling can be achieve by advancing or retracting the wire in the tunnel. Active stylets are beyond the scope of this work, and all channel considerations here will be in relation to a passive stylet tool. However, modifications could certainly be made to accommodate an active stylet.

For AOS insertions, a channel must be created in the array that is wide enough for placement of a stylet wire, yet small enough to fit inside the apical region of the guinea pig cochlea. Since the maximum widths of our guinea pig arrays are $280 \mu \mathrm{m}$ at the tip, we chose to use channel widths between $230 \mu \mathrm{m}$ and $250 \mu \mathrm{m}$, and $127 \mu \mathrm{m}$-diameter stainless steel stylet wires from Small Parts Incorporated. In an initial phase of fabrication, the array channels for the curled devices were fabricated as for the straight arrays (Fig. 3.27). Some curvature was achieved, and a stylet wire could be easily inserted through the channel (Fig. 3.31, Fig. 3.32, Fig. 3.33). However, the stiffness could not be sufficiently decreased to maintain the built-in curl, and post-processing 
methods had to be employed to increase curvature. Such methods included the use of a copper wire to induce additional strain into the arrays (Fig. 3.34).

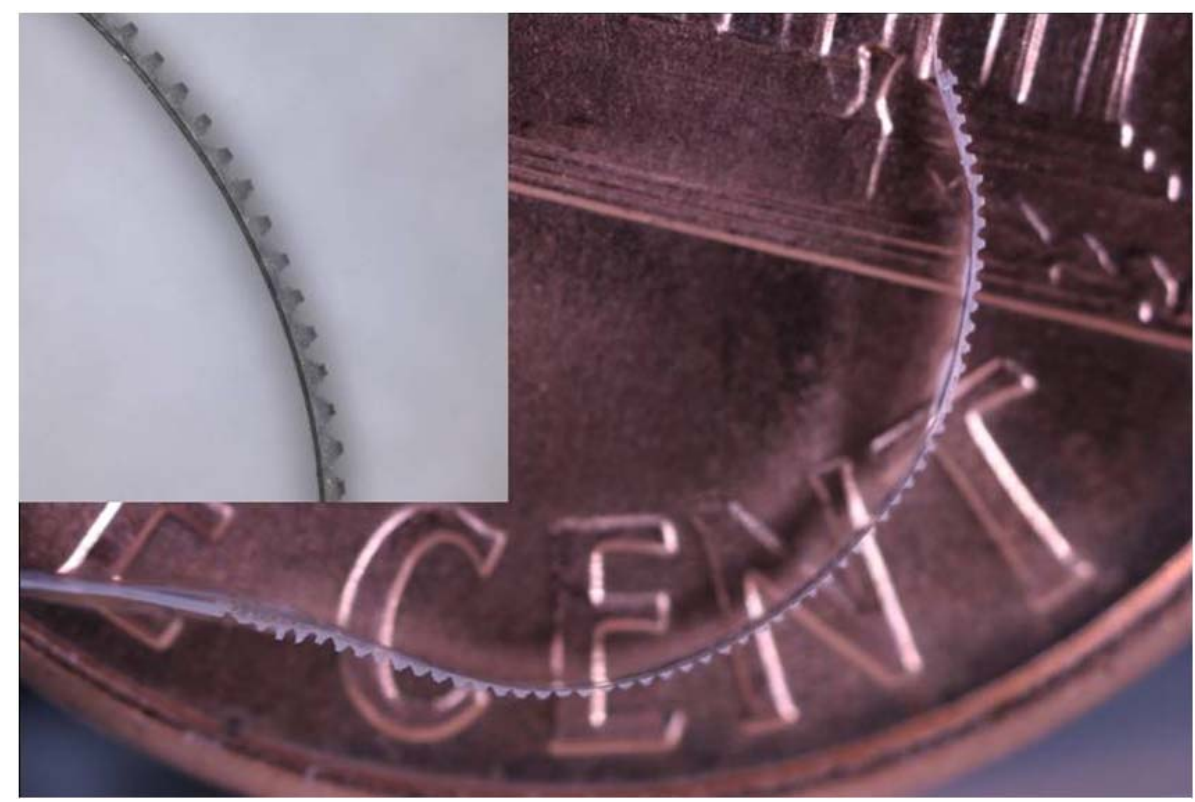

Fig. 3.31: Curled guinea pig array with parylene rings integrated on back.

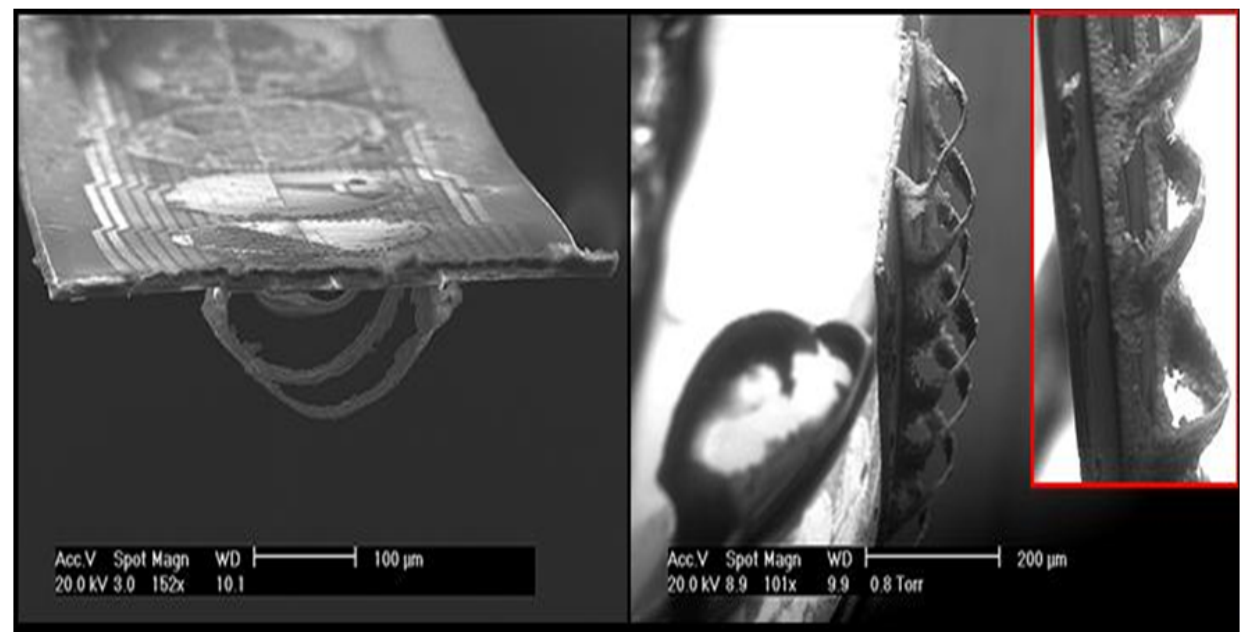

Fig. 3.32: Close-up of a curled guinea pig array with sites on one side and parylene rings on the other. 


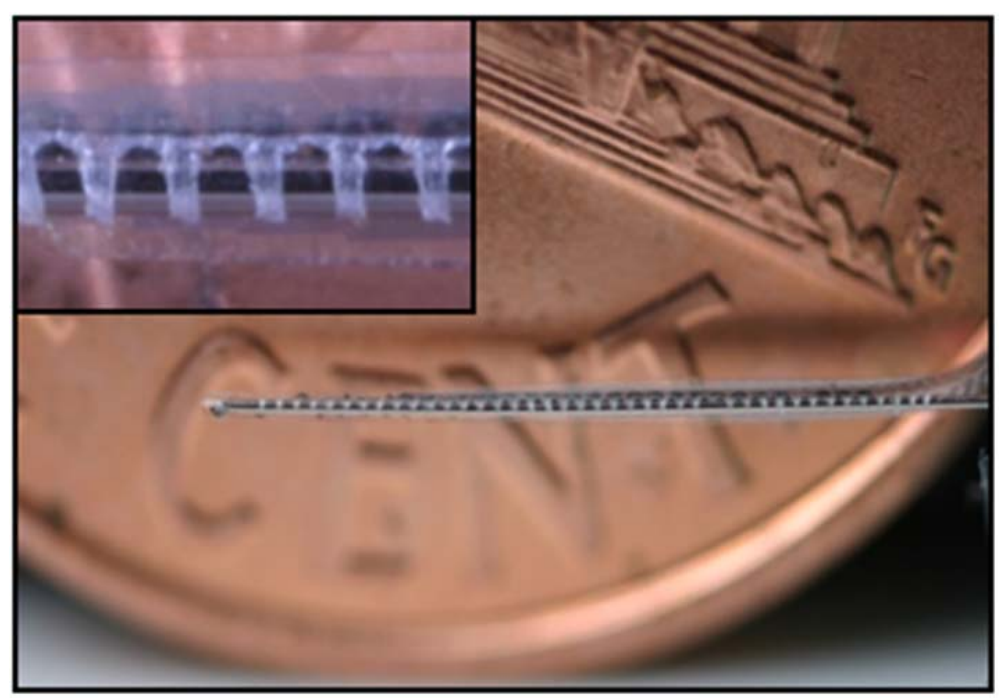

Fig. 3.33: A stylet-wire threaded through rings to straighten the pre-curved array for insertion into the cochlea.

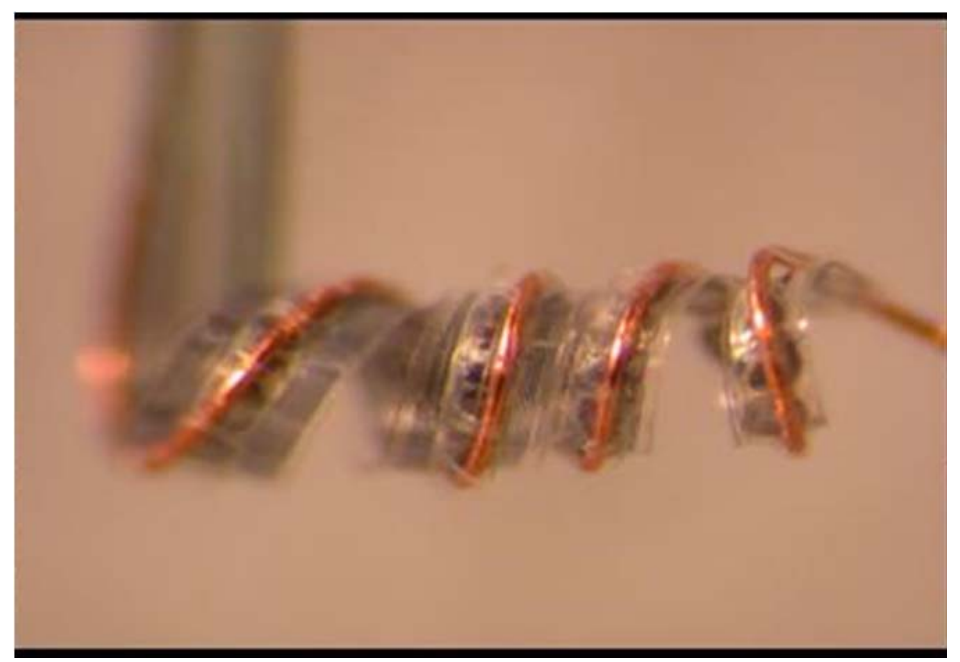

Fig. 3.34: A $100 \mu \mathrm{m}$-thick copper wire pre-curling guinea pig array.

The diminished curvature was a result of the residual gap wall height discussed previously. The gap walls created struts between the rings, which decreased curvature significantly. A new method of fabrication was investigated to create discrete rings that eliminated any gap wall.

FEA was conducted using the COMSOL Multiphysics software package to model changes in the ringed channel stiffness in relation to changes in $R_{L}$ and $G_{L}$. A 3D structural mechanics MEMS module was used. In the model, the channel is represented by a $200 \mu \mathrm{m}$-long, $300 \mu \mathrm{m}$-wide beam with attached rings. The thickness of the channel 
walls was $5 \mu \mathrm{m}$. An isotropic material model of parylene was used having a Young's modulus of $4 \mathrm{GPa}$, Poisson's ratio of 0.4 , and density of $1289 \mathrm{~kg} / \mathrm{m}^{3}$. The beam was fixed on one end and a perpendicular force of $416 \mathrm{~N} / \mathrm{m}^{2}$ was applied to the beam in the $\mathrm{x}$ direction, deflecting it upwards (Fig. 3.35). The stiffness $(k)$ of the channel was calculated from its deflection $(\delta)$ under the applied force $(\mathrm{F})$ using previously described equations (1) - (4).

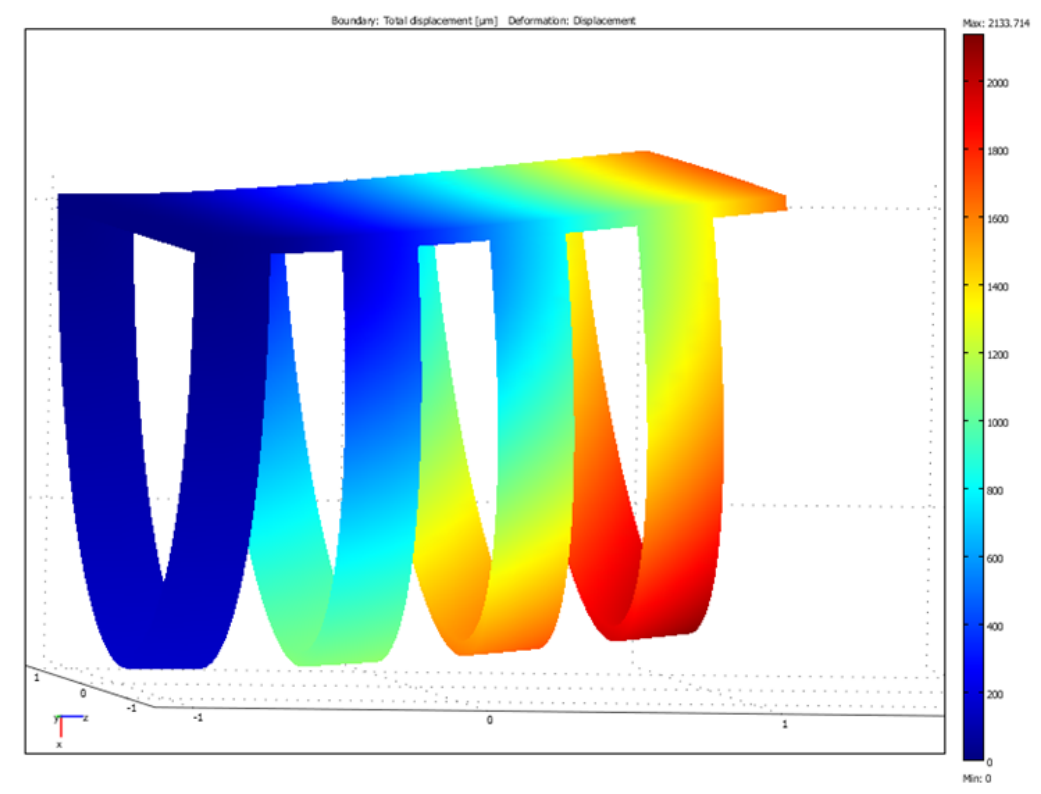

Fig. 3.35: A 3D FEA model of a discrete-ring channel.

As with the non-discrete rings, several simulations were done changing the ratios, values, and percentages of areas covered by $\mathrm{R}_{\mathrm{L}}$ and $\mathrm{G}_{\mathrm{L}}$. The goal was to determine how changing the parameters $R_{L}$ and $G_{L}$ changes the stiffness of the channel. Of those simulations, three types gave the best indication as to how stiffness varies with changes in $R_{L}$ and $G_{L}$. In one variation, the pitch covered by $R_{L}$ and $G_{L}$ was kept constant and $G_{L}$ was increased. In the next, the pitch covered by $R_{L}$ and $G_{L}$ was kept constant and the ratio of $R_{L} / G_{L}$ was increased. The last variation entailed keeping the ratio $R_{L} / G_{L}$ constant and increasing both $\mathrm{R}_{\mathrm{L}}$ and $\mathrm{G}_{\mathrm{L}}$.

The stiffness of the channel decreases as $\mathrm{G}_{\mathrm{L}}$ increases for situations in which the pitch is kept constant (Fig. 3.36, Fig. 3.37). For this case, the percentage of the total area covered by gaps goes up and thus there are less fixed regions and greater flexibility. 


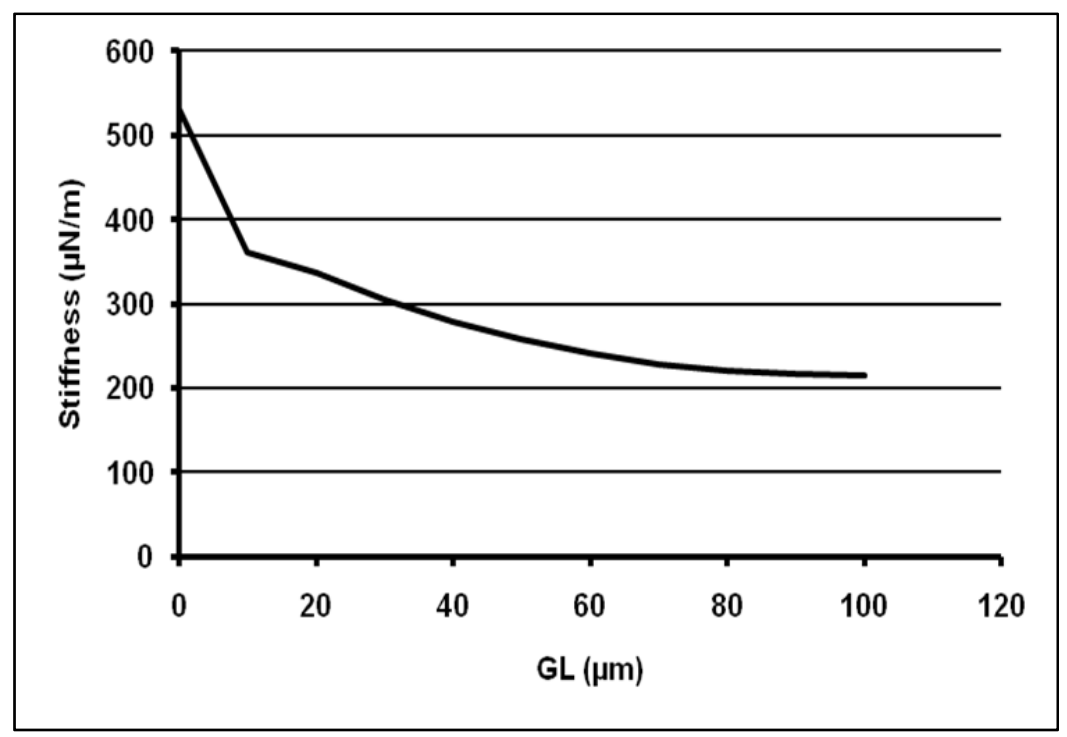

Fig. 3.36: The stiffness of ringed-channel devices versus the ring gap length for a constant ring pitch of a $100 \mu \mathrm{m}$.

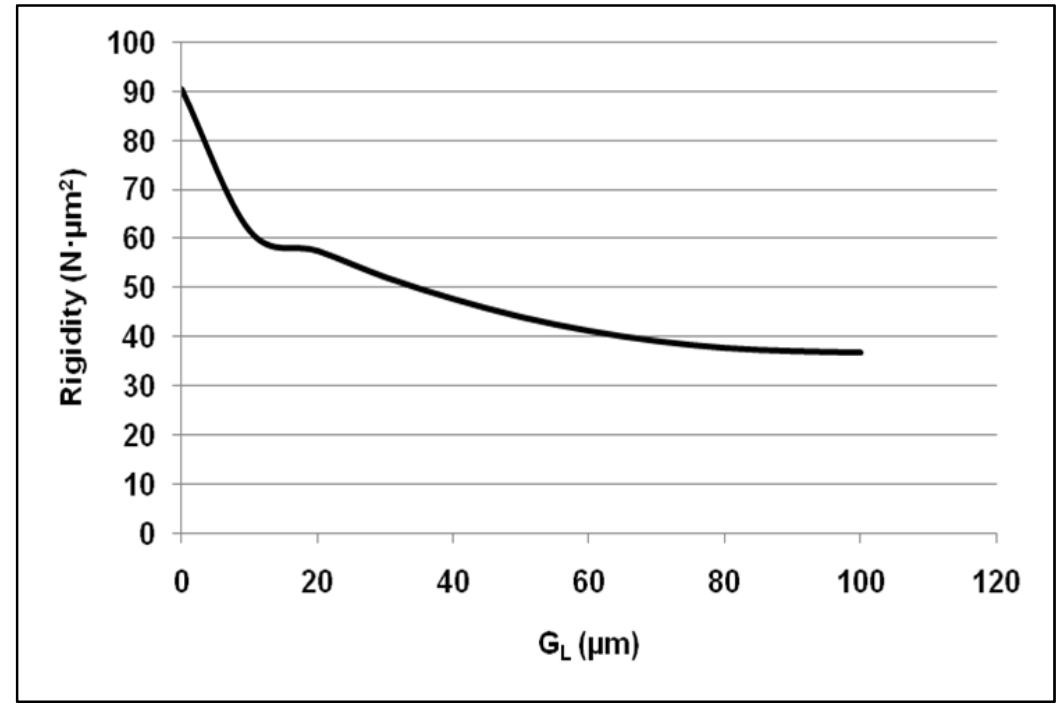

Fig. 3.37: The rigidity of a ringed-channel device versus the ring gap length for a constant ring pitch of a $100 \mu \mathrm{m}$. 


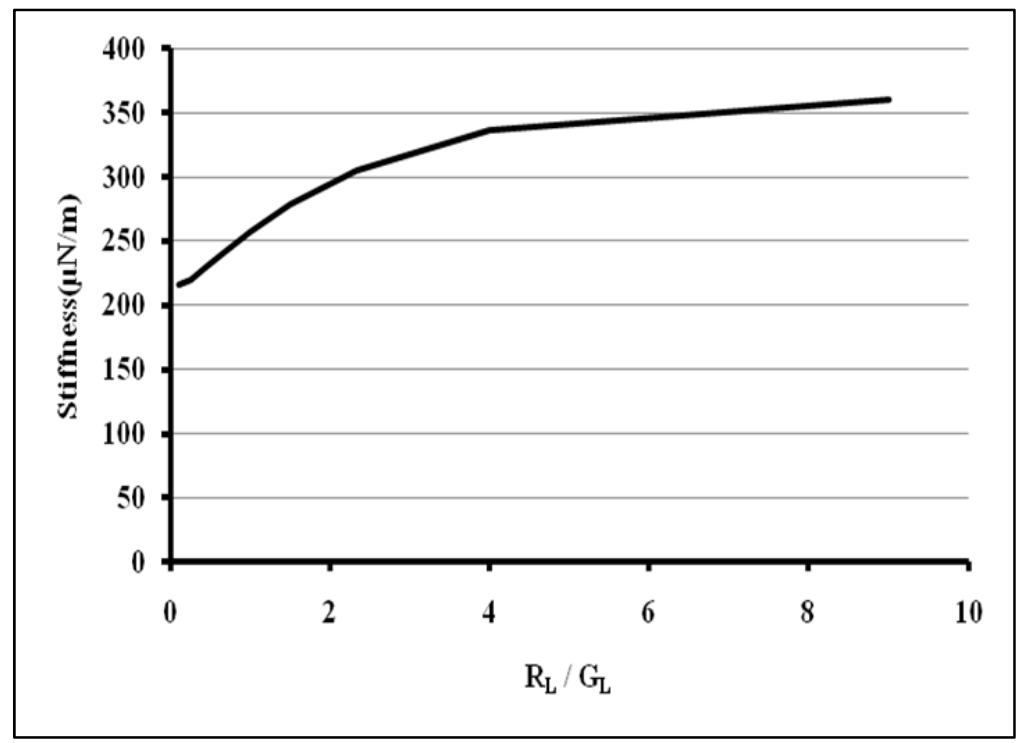

Fig. 3.38: The stiffness versus the ratio of $\mathrm{R}_{\mathrm{L}} / \mathrm{G}_{\mathrm{L}}$ for a constant ring pitch of 100 $\mu \mathrm{m}$.

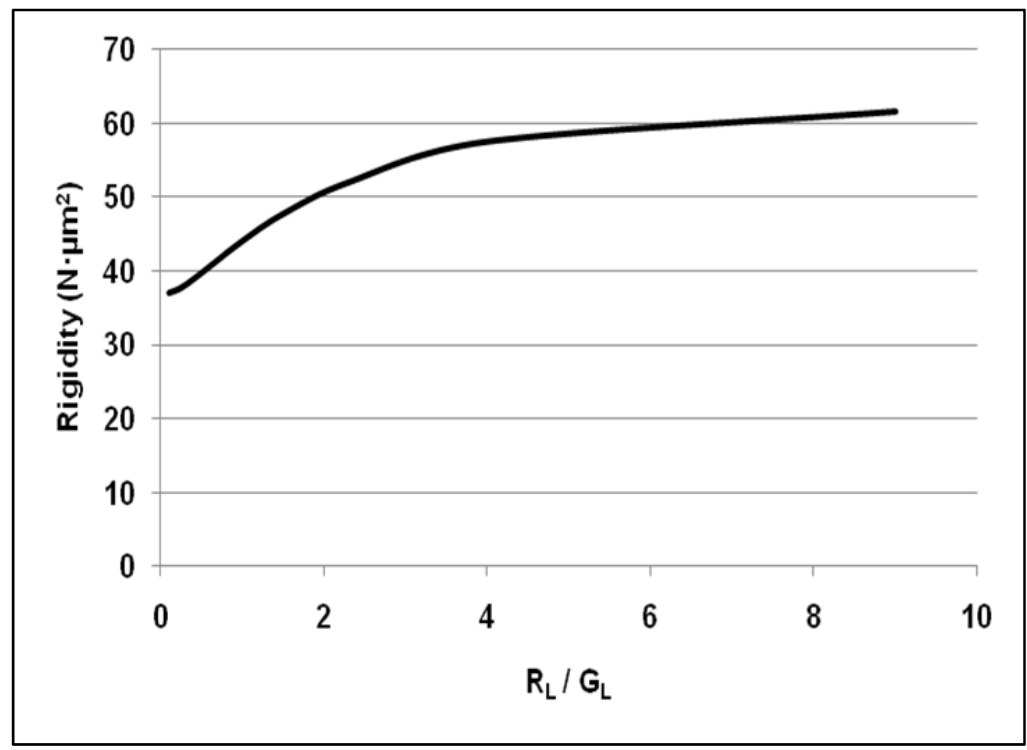

Fig. 3.39: Rigidity versus the ratio of $\mathrm{R}_{\mathrm{L}} / \mathrm{G}_{\mathrm{L}}$ for a constant ring pitch of $100 \mu \mathrm{m}$.

For constant pitch, the stiffness of the backing increases with increases in the ratio of $\mathrm{R}_{\mathrm{L}} / \mathrm{G}_{\mathrm{L}}$ (Fig. 3.38, Fig. 3.39). The FEA results also suggest that stiffness increases for situations where the ratio of $\mathrm{R}_{\mathrm{L}} / \mathrm{G}_{\mathrm{L}}$ is kept constant and $\mathrm{R}_{\mathrm{L}}$ is increased (Fig. 3.40, Fig. $3.41)$. 


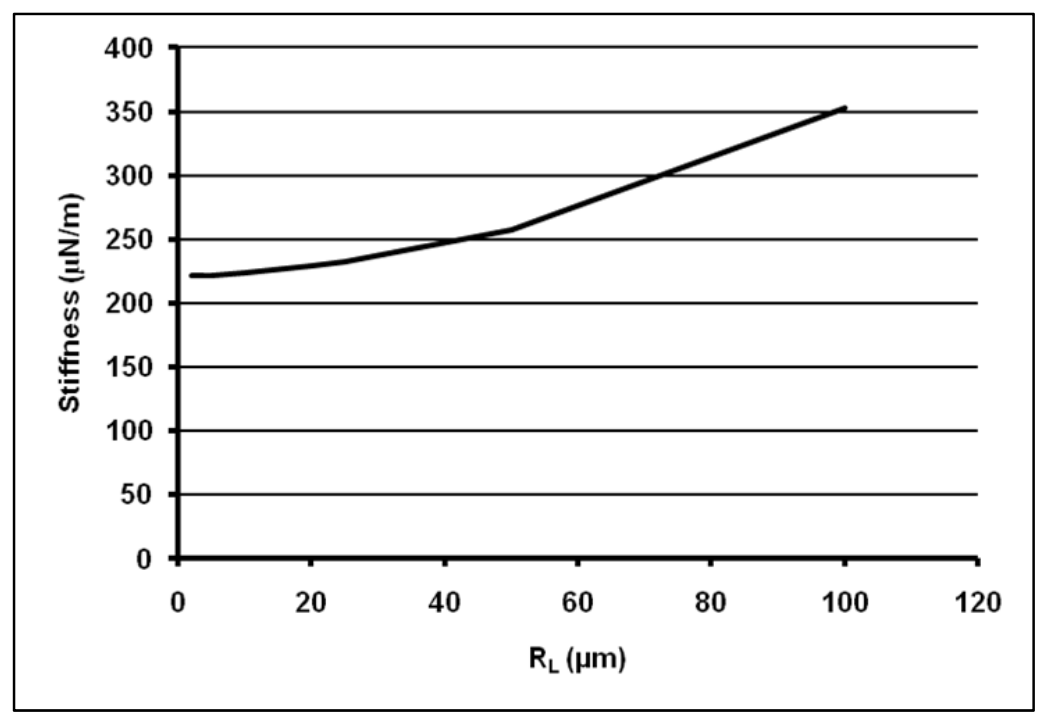

Fig. 3.40: Stiffness versus the ring length for a constant $R_{L} / G_{L}$ ratio of 1 .

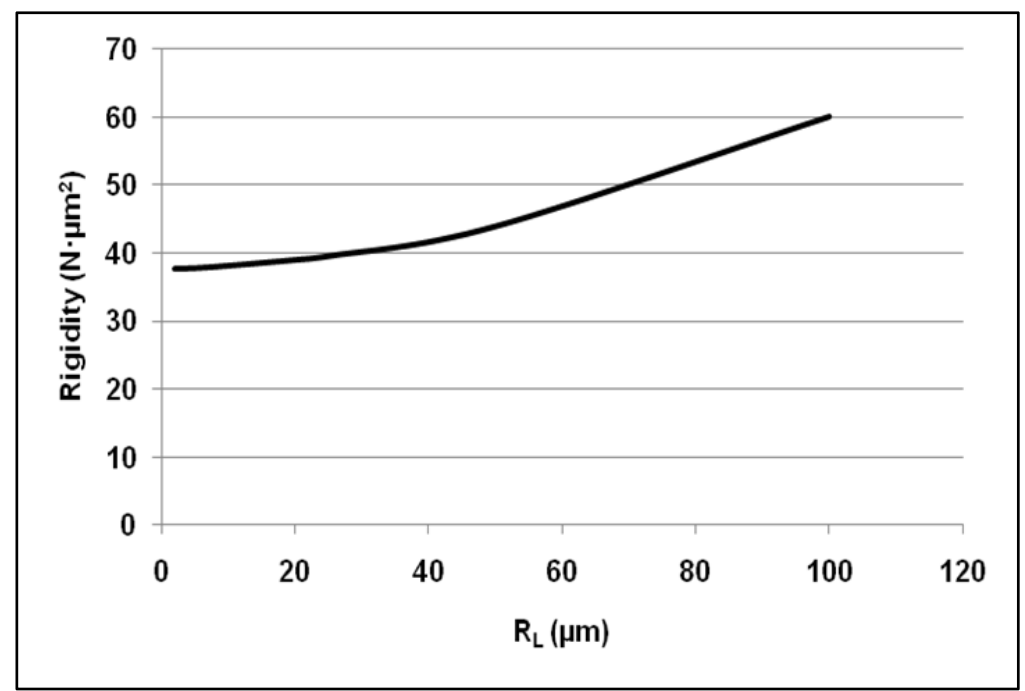

Fig. 3.41: Rigidity versus the ring length for a constant $\mathrm{R}_{\mathrm{L}} / \mathrm{G}_{\mathrm{L}}$ ratio of 1 .

With the above simulations it can be seen that stiffness can be defined with near linear control. This implies that a graded stiffness can be added to the array if desired to control the degree of curvature in specific regions of the device. The range of stiffness varied from $550 \mu \mathrm{N} / \mathrm{m}$ for a continuous channel to $200 \mu \mathrm{N} / \mathrm{m}$ for a parylene substrate with no rings. Several ring and gap length combinations can be used to add rings to a parylene substrate without significantly increasing the stiffness. According to the model, a channel with a ring length of $30 \mu \mathrm{m}$ and gap length of $70 \mu \mathrm{m}$ can be fabricated with high yield, and has a stiffness of $230 \mu \mathrm{N} / \mathrm{m}$, which is only $15 \%$ 

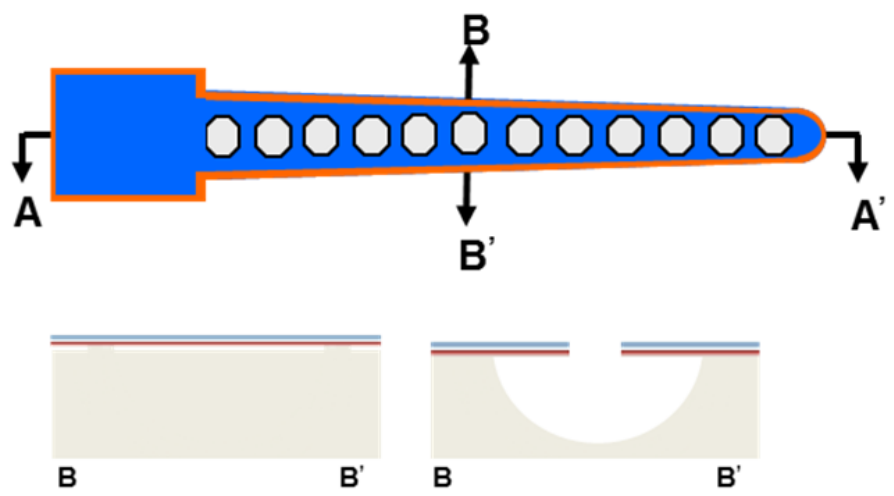

(a)

(b)

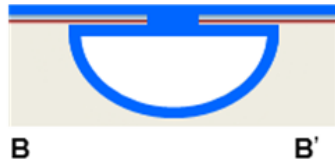

(c)

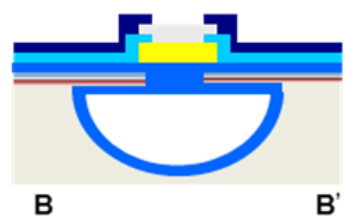

(d)

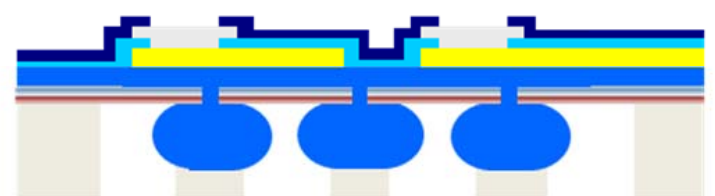

A

A'

(e)

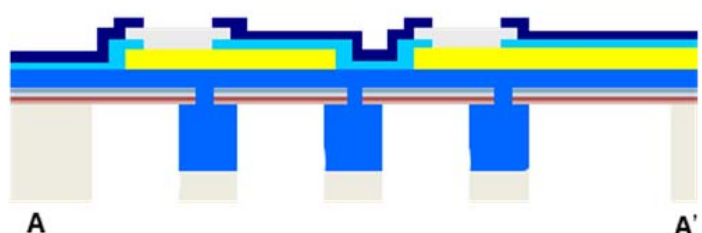

(f)

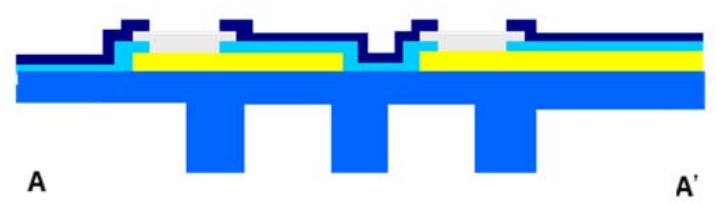

(g)

$\begin{array}{lll}\text { Carrier Substrate } & \text { ParyleneTube } & \text { Enclosure } \\ \text { Tunnel Overlap } & \text { Curl Strips } & \text { Metal Interconnect } \\ \text { Insulation } & \text { Metal Sites } & \text { Oxide Etch-Stop }\end{array}$

Fig. 3.42: The fabriation process flow for discrete-ring devices. 
greater than that of a channel with no rings. Although many rings could be produced with little effect on curvature, for the ease of fabrication and to improve process yields, arrays were fabricated with $100 \mu \mathrm{m}$ wide rings spaced $1 \mathrm{~mm}$ apart. The fabrication process for the discrete-ringed arrays follows that of the slotted channel (Fig. 3.42). Fabrication begins with the vapor deposition of a thin $2 \mu \mathrm{m}$ masking layer of parylene over a PECVD oxide etch-stop (Fig. 3.42a). A series of small squares, $5 \mu \mathrm{m}$ wide and 50 $\mu \mathrm{m}$ long, extending the length of the shank $(11 \mathrm{~mm})$ and spaced $1 \mathrm{~mm}$ apart are etched out of the parylene using a directional $\mathrm{O}_{2}$ plasma. Following the selective removal of parylene, the remaining overlap is isotropically undercut $100 \mu \mathrm{m}-150 \mu \mathrm{m}$ to form a channel using a $\mathrm{XeF}_{2}$ etch (Fig. 3.42b). The channel walls are then coated with parylene and the overlap opening is sealed off (Fig. 3.42c). Due to the small width of the parylene opening, the channel is can be completely sealed by depositing a $7 \mu \mathrm{m}$ layer of parylene. Once the discrete channels are filled in, the parylene stimulating array is built on top of it (Fig. 3.42d). Next, the backside of the silicon wafer is selectively masked and etched using DRIE (Fig. 3.42e). The oxide provides an etch-stop to prevent the parylene substrate from being etched. Following this step, the exposed regions of the tunnel are removed in a directional $\mathrm{O}_{2}$ plasma etch (Fig. 3.42f). Lastly, the entire array is released from the carrier wafer using a $2 \% \mathrm{KOH}$ solution (Fig. 3.42g).

The discrete-ring process was integrated with the PIP curvature control technique to produce curved arrays with monolithic backings (Fig. 3.43). The arrays achieved full insertion inside GP cochlea molds, hugging the modiolus wall (Fig. 3.44, Fig. 3.45). 


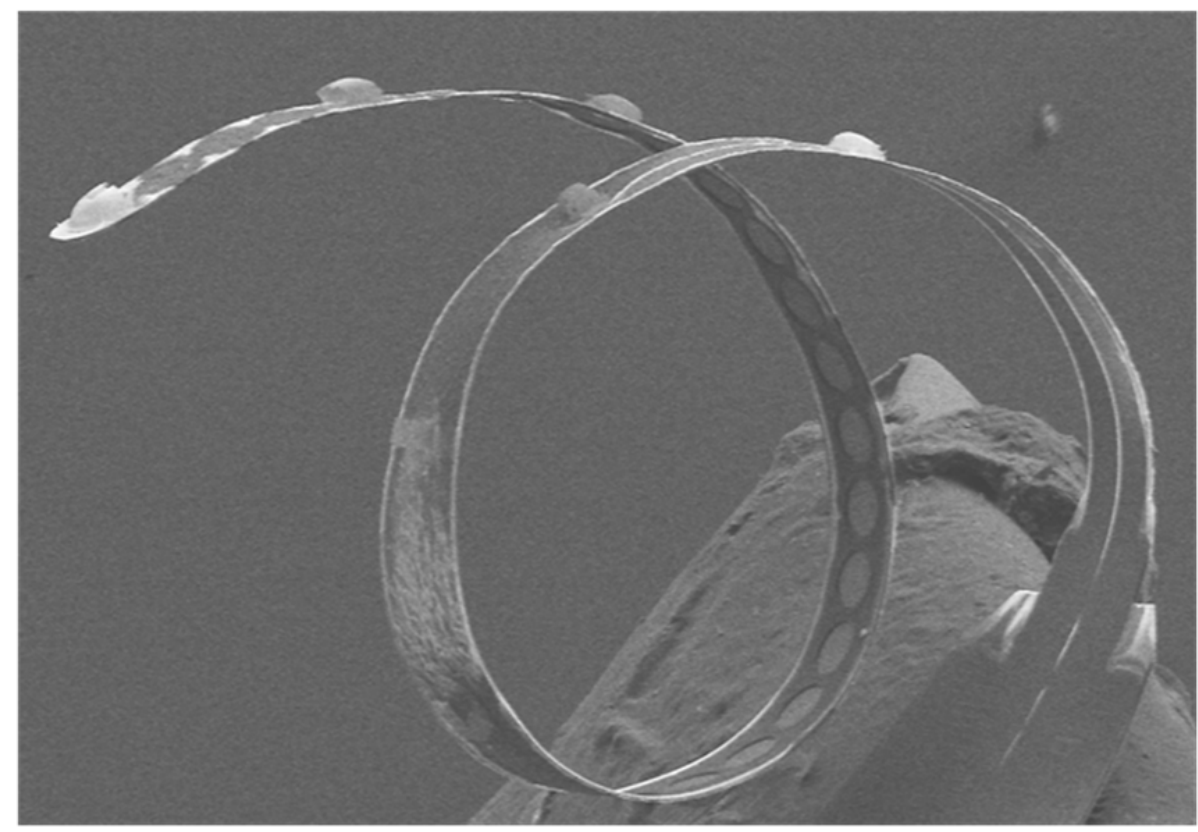

Fig. 3.43: A pre-curved parylene array, backed with discrete rings.

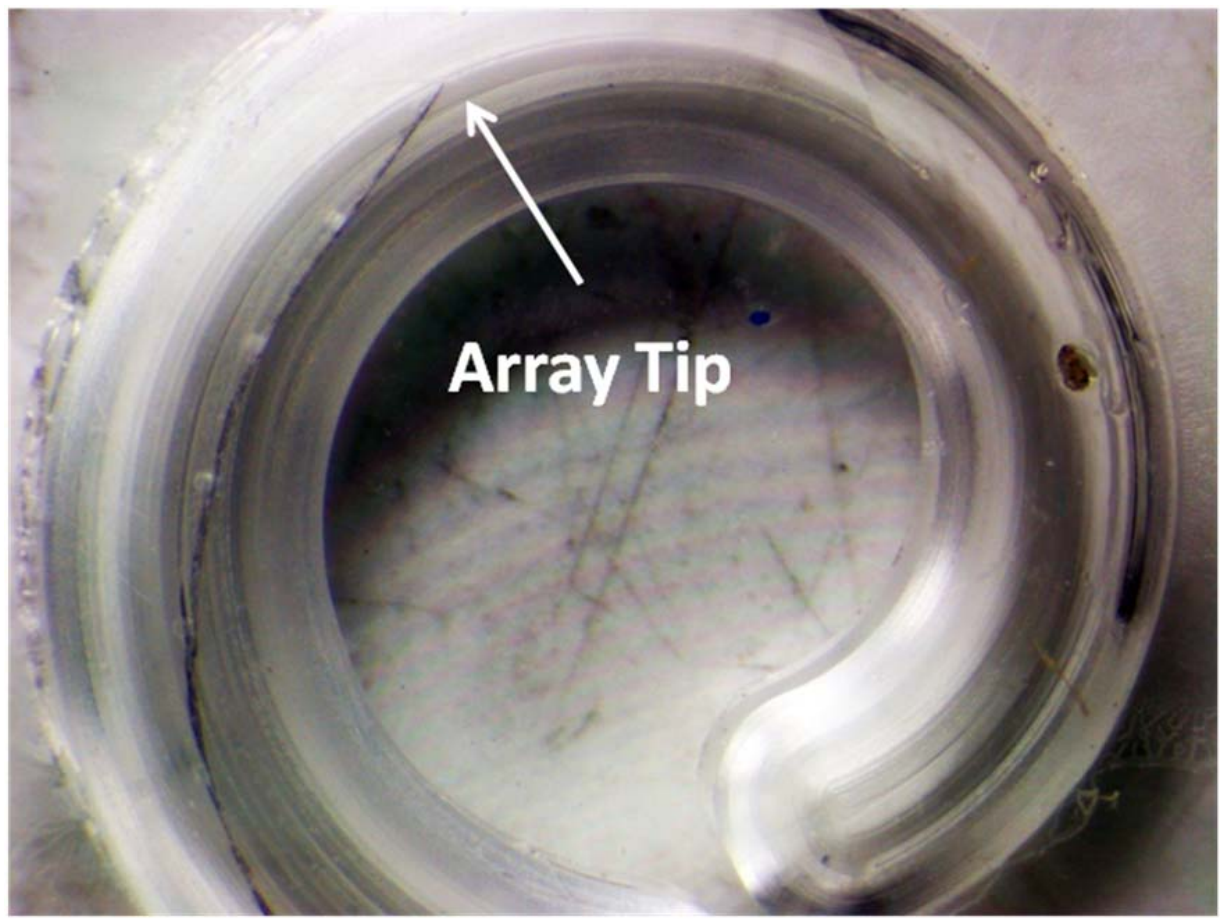

Fig. 3.44: A straight ringed array going around the first turn of the cochlea. Having no curvature the device does not hug the modiolus. 


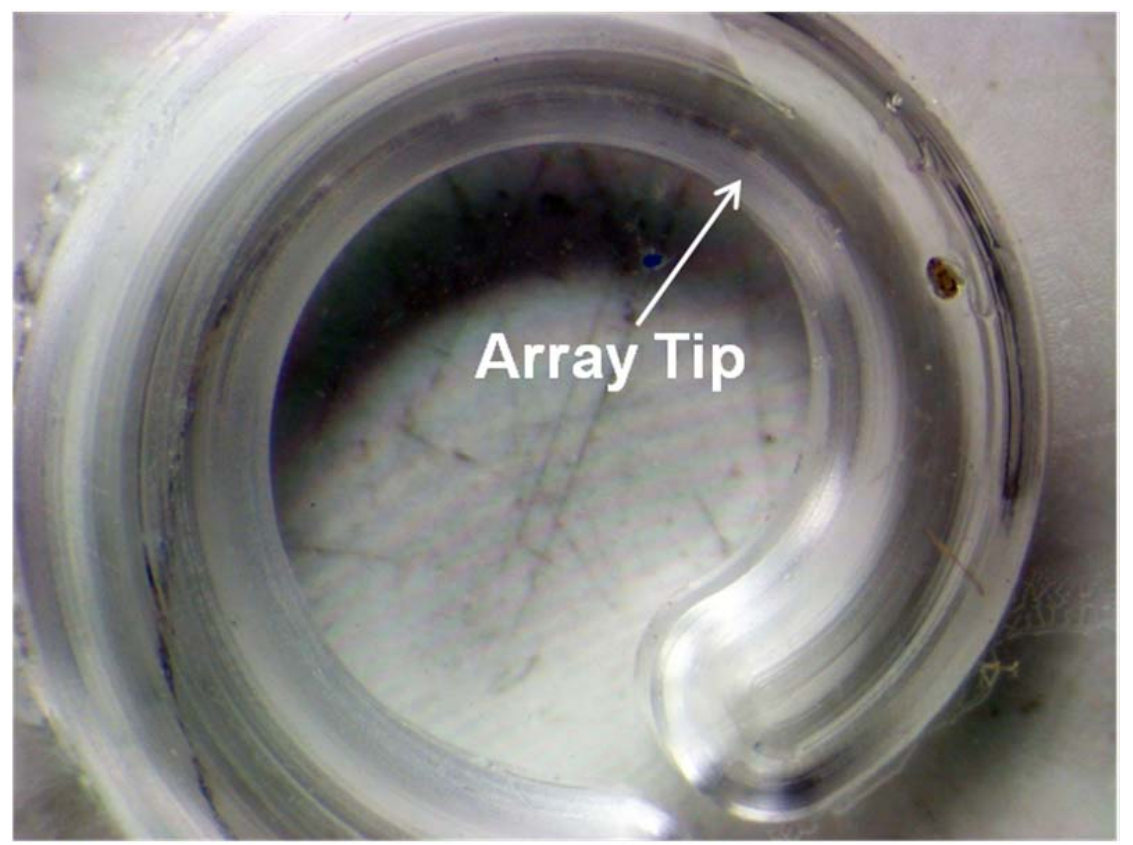

Fig. 3.45: A pre-curved parylene array inserted through a cochlea mold. The curvature of the array allows it to hug the modiolus wall.

\subsubsection{Backing Summary}

Molded and thin-film backing structures have been investigated for incorporation onto the parylene stimulating arrays. Molded backings have been applied by both UCSF and Cochlear Ltd. The silicone carriers added by Cochlear are the same as the ones used for their Nucleus Contour arrays. The Contour arrays are the most popular implants in use today. As such, incorporation of the parylene arrays into these carriers could lead to a quicker and greater acceptance of the thin-film devices in the medical market. The drawback of the silicone carriers, however, is that there is limited control over the mechanical properties of the array. A better approach will be to use a combination of silicone molding and monolithic thin-film backings. In the area of thin-film backings, both hybrid and monolithic types were designed and implemented. A hybrid approach using PET tubing was investigated by MTU, and provided full insertion of the parylene arrays into a cat cochlea during in-vivo testing. However, the process of attaching the PET produced low yield, and delamination of the arrays from the PET backing during insertion was common. As an alternative, monolithic backings were developed to produce curl and stiffness over the array. Curl in the array was produced with residually 
stressed parylene and produced curvature radii of less than $0.5 \mathrm{~mm}$. Monolithic ringedchannels were integrated into the back of the parylene arrays to control stiffness and for use with the AOS technique. A slotted ring structure was used to stiffen the straight arrays. Simulated channel rigidity had a maximum of $1.4 \mathrm{kN} \cdot \mu \mathrm{m}^{2}$ and a minimum of 0.2 $\mathrm{kN} \cdot \mu \mathrm{m}^{2}$. A discrete ring structure was implemented on pre-curved arrays to provide a flexible channel for stylet placement. The ring-backed pre-curved arrays hugged the modiolus wall of in-vitro cochlea molds and was suitable for use with AOS insertion. This type of monolithic backing, alone or incorporated with a silicone carrier, will greatly improve the usability, reliability, and cost of thin-film cochlear electrode arrays.

\subsubsection{References}

[1] Wang, J.; Gulari, M. N. ; Wise, K. D., "A Parylene-Silicon Cochlear Electrode Array with Integrated Position Sensors," 28th IEEE International Conference on Engineering in Medicine and Biology Society, pp. 3170-3173, 2006

[2] Merzenich, M. ; Byers, C. L.; White M.; Vivion, M. C., "Cochlear implant prostheses: strategies and progress," Annals of biomedical engineering, vol. 8, pp. 361,1980

[3] Rebscher, S.J.; Hetherington, A. M.; Snyder, R.L.; Leake, P.A.; Bonham, B.H., "Design and fabrication of multichannel cochlear implants for animal research," Journal of Neuroscience Methods, vol. 166, pp. 1-23, 2007

[4] Arcand, B. Y.; Bhatti, P. T.; Butala, N. V.; Wang, J., Friedrich, C. R.; Wise, K. D., "Active positioning device for a perimodiolar cochlear electrode array," Microsystem technologies, vol. 10, pp. 478, 2004

[5] Wang, J.; Gulari, M.; Bhatti, P.T.; Arcand, B.Y.; Beach, K.; Friedrich, C.R.; Wise, K.D.; , "A cochlear electrode array with built-in position sensing," 18th IEEE International Conference on Micro Electro Mechanical Systems , pp. 786- 789, 2005

[6] Tewari, R.; Friedrich C., "Hot Embossing of Poly (Lactic Acid) films for an Embedded Cochlear Implant Stiffener.", Journal of Microsystem Technologies, vol. 16, pp. 1601-1607, 2010

[7] Dabral, S.; Van Etten, J.; Zhang, X.; Apblett, C.; Yang, G.; Ficalora, P.; McDonald, J., "Stress in thermally annealed parylene films." , Journal of Electronic Materials, vol. 21, pp. $989-994,1992$ 
[8] Roland, J.T., "A model for cochlear implant electrode insertion and force evaluation: results with a new electrode design and insertion technique," The Laryngoscope, vol. 115, pp. 1325 - 1339, 2005.

[9] Chen, P. J.; et al., "Unpowered spiral-tube parylene pressure sensor for intraocular pressure sensing," Sensors and actuators A: Physical, vol. 127, pp. 276 - 282, 2006.

[10] Chen, P.-J. ; Shih, C.-Y.; Tai, Y.-C., "Design, fabrication and characterization of monolithic embedded parylene microchannels in silicon substrate," Lab on a chip, vol. 6, pp. 803 - 810, 2006.

[11] Meng, E.; Tai, Y.C. , "Parylene etching techniques for microfluidics and bioMEMS,", 18th IEEE International Conference on Micro Electro Mechanical Systems , pp. 568- 571, 2005 


\section{Chapter 4}

\section{IN-VIVO PERFORMANCE}

Following the fabrication of each electrode array, in-vitro characterization is done by measuring site back voltages and impedances and by inserting the arrays through acrylic models of the cochlea. The in-vitro characterization provides some insight into how the arrays will work in practice, but it is critical to do in-vivo insertions and stimulation to really characterize the array performance. The goal of the in-vivo experiments was to show that the arrays can be fully implanted into live mammalian cochleae, can be operated at threshold levels better than or comparable to those of handassembled arrays, and can elicit comparable neural responses to intracochlear stimulation at both threshold and suprathreshold current levels.

Cat and guinea pig mammalian models were used to characterize the in-vivo performance of the parylene cochlear electrode arrays. In total, twelve guinea pigs and two cats were implanted. Four different parylene array types were tested in vivo: a cat electrode array backed with a flattened, pre-curved PET tube (PC), a guinea pig cochlear electrode array backed with parylene rings having a graded stiffness (GS), a guinea pig cochlear electrode array backed with a ringed-channel of uniform stiffness (US), and a guinea pig cochlear electrode array with monolithically-controlled curl and ringed array backing (MC). The arrays were assessed both mechanically and physiologically. Mechanical assessment was done through a series of live animal and cadaver insertions. Physiological assessment was done using both Electrically Evoked Auditory Brainstem Responses (EABRs) and recordings from the Inferior Colliculus (IC) to determine the stimulus current thresholds for the arrays and their ability to elicit auditory responses. In the following sections, the implant procedure and experimental results will be discussed. 


\subsection{Surgical Procedure}

Cat and guinea pig surgeries were conducted under similar surgical procedures. The guinea pig surgical procedure will be discussed in detail here. All animal surgeries were conducted at the University of Michigan Kresge Hearing Research Institute. The cat implantations were conducted with the aid of Dr. John Middlebrooks of The University of California at Irvine and Dr. Russell Snyder of the University of California at San Francisco. The guinea pig implantations were conducted with the aid of Dr. Bryan Pfingst and Ms. Deborah Colesa of the University of Michigan Department of Otolaryngology.

About 1-2 hours before any before any surgical procedure begins, to ensure the animals remain well maintained throughout the duration of the implant, the animals are given $2 \mathrm{~mL} / 100 \mathrm{~g}$ of warm sodium chloride subcutaneously to keep their blood pressure up. Throughout the duration of the procedure the animals are anesthetized with ketamine $(40 \mathrm{mg} / \mathrm{kg})$ and xylazine $(10 \mathrm{mg} / \mathrm{kg})$. The animal's breathing patterns are repeatedly monitored, and Atropine $(0.05 \mathrm{mg} / \mathrm{kg})$ is administered to help with respiration. The guinea pigs are placed on a circulating hot water heating pad and monitored with a temperature probe. Ophthalmic ointment is applied to the eyes throughout the surgery to prevent drying, and Lidocaine is injected along the incision site to prevent the animal from feeling any pain.

Before a cochleostomy is created for inserting the electrode array, a dental burr is used to drill five $1.5 \mathrm{~mm}$ holes with three around the bregma of the skull. Three stainless steel screws are use to secure an inverted restraint bolt to the animal skull. This restraint bolt and two additional stainless steel screws are ultilized for EABR recording (Fig. 4.1). Methyl methacrylate is coated over all six screws to fix them in place. 


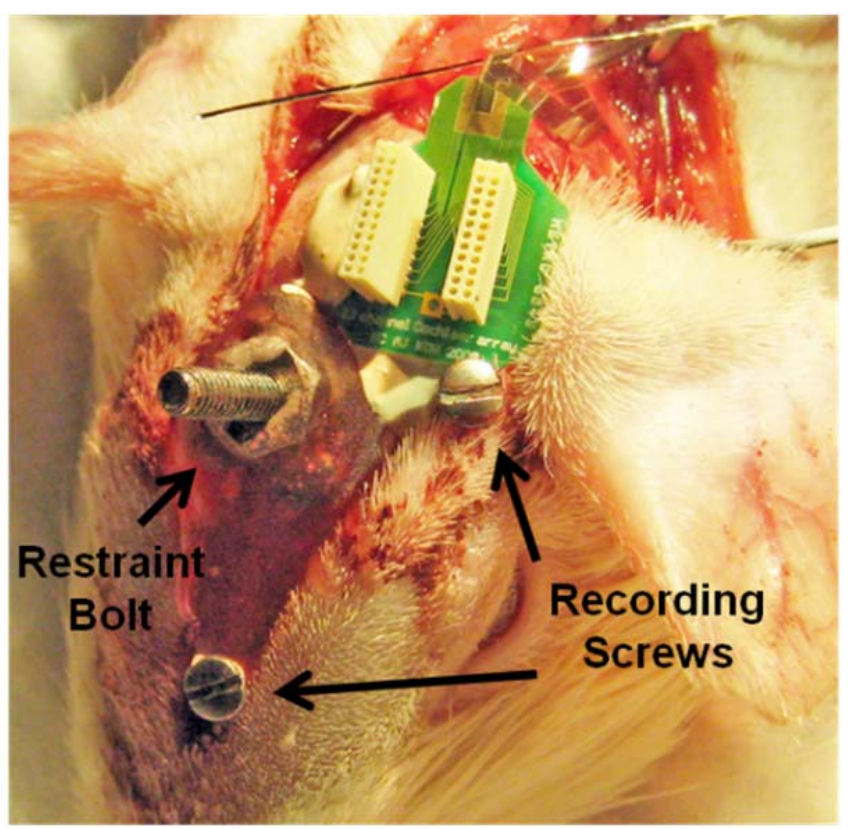

Fig. 4.1: Three electrode screws for EABR recording including ground, reference and active electrodes.

Following placement of the screws around the bregma of the skull, the animal is rotated to a lateral position, and an incision is made behind the ear to be implanted. The soft tissue over the bulla is dissected to expose the temporal bone. A scaple blade is used to create a small defect in the bulla, and this defect is carefully widened until the round window can be clearly visualized. Next, a small cochleostomy is made in the basal turn of the cochlea (just below the round window) using a \#60 diamond burr to allow for insertion of the implant. The electrical connector of the parylene implant is secured to the skull with durelon cement, making sure not to over extend or unduly twist the parylene cable connected to the array. Once the cement has thoroughly dried, the electrode array is inserted gently into the scala tympani through this cochleostomy using direct placement with a pair of tweezers or by advancing the array off of a stylet using the AOS technique (Fig. 4.2). 


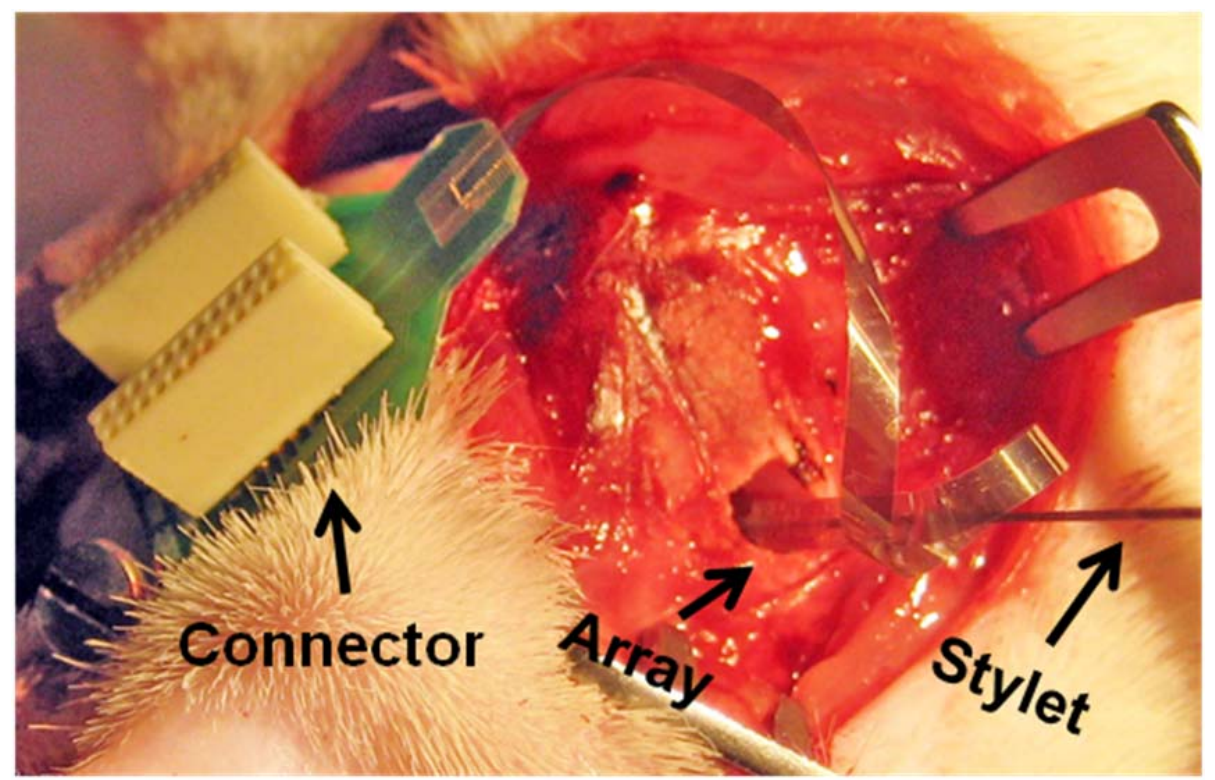

Fig. 4.2: Array positioned at entrance to cochlea through the bulla defect. A connector mounted on skull provides electrical access to the array sites.

In either version of insertion, the implant is fed along the top of the scala tympani opening to prevent snagging the implant tip on the first turn and to allow it to advance easily into the second turn. Once fully implanted, the implant is secured at the bulla defect with dental cement (Durelon).

An IC recording is performed following the cochlear implant surgery. For insertion of the IC recording electrode array, a craniotomy is performed to expose the visual cortex overlying the IC. Before making any incisions, the skull is fixed with a high degree of stability to a rigid bar by the restraint bolt on the bregma. Following fixation, the dura is removed, and the cortex is aspirated to visually see the IC for insertion of an acute 16-channel Neuronexus probe. The probe is inserted into the IC at a $45^{\circ}$ angle relative to the sagittal plane of the skull using a stereotaxic manipulator. The implant is inserted approximately $3 \mathrm{~mm}$ into the IC.

\subsection{Stimulation and Recording Setup}

During EABR recording, neural activity was recorded using alligator clips attached to active and reference electrodes (screws) in the animal's headset. The ground 
electrode for recording was the restraint bolt placed on the bregma. The ground electrode for the cochlear electrode array was tucked into the muscle overlying the temporal bone.

The anesthetized animals were stimulated with $25 \mu \mathrm{s}$ or $200 \mu$ s duration monophasic, charge balanced, cathodic first pulses at 50 pps, with 2048 pulses per trial. To obtain an input-output function, stimulus levels were adjusted from the maximum comfort level to subthreshold. The beginning amplitude tested was set to a standard comfortable level for monopolar stimulation $(300 \mu \mathrm{A})$, and then the stimulus was gradually decreased in steps of $50 \mu \mathrm{A}$ until no response was present and then increased above $300 \mu \mathrm{A}$ up to the maximum level that resulted in pina/facial movement. Electrical stimuli were generated by a Tucker Davis digital-to-analog converter (DA3-2), fed through a controlled constant-current stimulator with a maximum output of $1 \mathrm{~mA}$ peakto-peak. The stimulator output was connected to the implant through the connector mounted on the animal's skull. Neural activity was amplified with a Grass Instruments bioamplifier set to a gain of 10,000 and a bandwidth of $0.1-3 \mathrm{kHz}$. Following amplification, the analog activity was converted to a digital signal using a Tucker Davis AD1 analog-to-digital converter. Stimulation was set and neural activity was recorded using the software package BioSig 32 stimulator record (version 3.12). EABR recordings can take on many shapes and have a variable number of voltage peaks (P1, P2, P3, etc.) depending on what stimulus, animal model, and place of stimulation are used. Voltage peaks that appear in the EABR responses can be stimulus artifacts, muscle responses or neural responses to stimulation. Only neural responses to stimulation are relevant when determining threshold levels for the cochlear electrode array. To determine whether or not the peak observed was in response to auditory stimulation, the peak was tracked over a series of current levels and its time of occurrence was noted. Peaks that advanced in time and occurred within a 1.3-2.5 ms time window were relevant. All EABR recordings used in the guinea pig implant studies had three positive peaks (P1, P2, and P3) followed by corresponding negative peaks. The first peak, P1, was typically overshadowed by a stimulus artifact. P2 and P3 were neural responses to intracochlear stimulation. Of P2 and P3, only one was used to determine the threshold level of the array, whichever was more prominent. This peak was measured and followed over time to assess threshold. Peak amplitude was either the distance from the zero line to the top of the positive peak 
or the difference between the most prominent positive peak and the negative peak preceding it.

Biphasic stimulus pulses with $200 \mu$ s phase durations were generated at a rate of 4pps by the program Sig Play 32 (version 3.3). The digitally generated signals of Sig Play were connected to a Tucker Davis D/A converter and an attenuator whose output was connected to an ISSRC6 isolated current source $(100 \mu \mathrm{A} / \mathrm{V})$. Neural activity in response to stimulation was amplified with a Plexon recording box set to a gain of 20,000 and a bandwidth of 1-20 kHz. The signals were then collected using NeuroExplorer (version 2.660) as continuous spike activity and histograms of nerve firing over time. Standard sixteen-site Neuronexus recording probes were used to pick up neural activity in the IC.

\subsection{Mechanical Performance}

All four-electrode array types (PC, MC, US, and GS) were mechanically inserted into the cochleae of living animals. The goal of these insertions was to assess the mechanical nature of the arrays during implantation and to prove that the ultra-flexible devices could be fully inserted into mammalian cochlea. Post-mortem dissections were done following each implant to determine the exact placement of the inserted array.

The PET-backed cat cochlear electrode arrays (PCs) were implanted into the cat cochlea using the AOS method. A stainless-steel stylet wire was inserted through the PET channel on the back of the array, and the entire assembly was inserted just beyond the cochleostomy into the scala tympani (Fig. 4.3). 


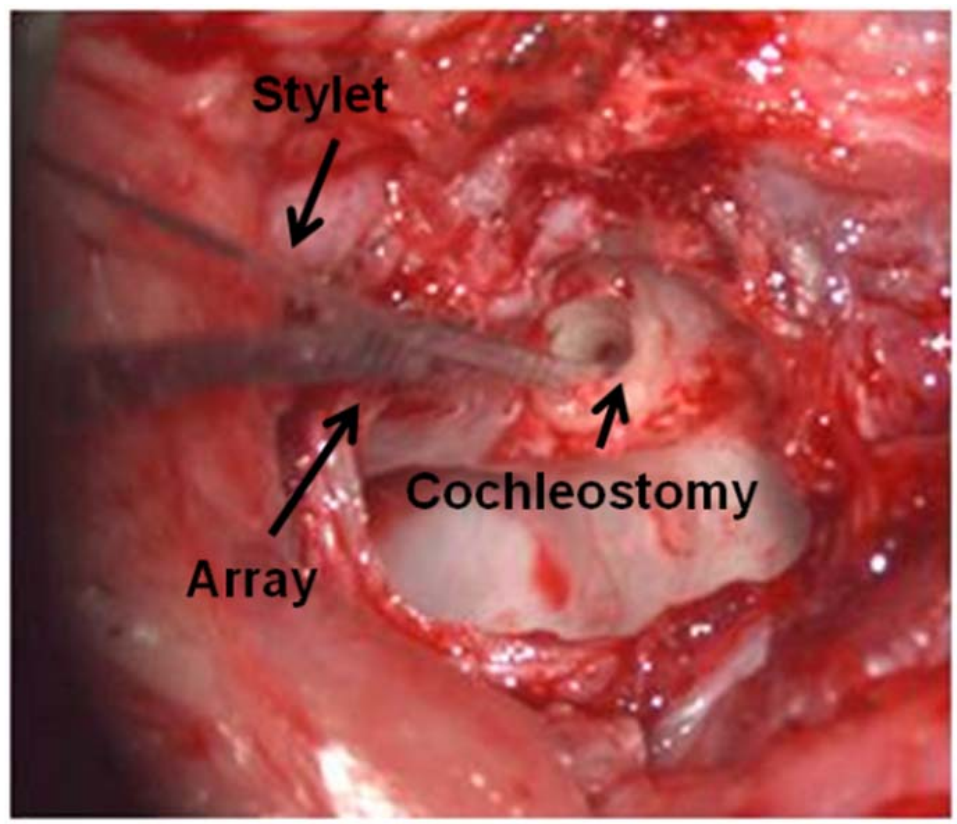

Fig. 4.3: The PC array at the entrance to cat cochlea.

A micromanipulator was then used to grip the stylet wire, while the array was slowly advanced off of the stylet beyond the first turn of the cochlea and into the second turn. Dissections revealed that the array went in beyond the first turn, but at its midpoint the shank actually cut through the scala media, a gel-like membrane, and entered the scala vestibule (Fig. 4.4).

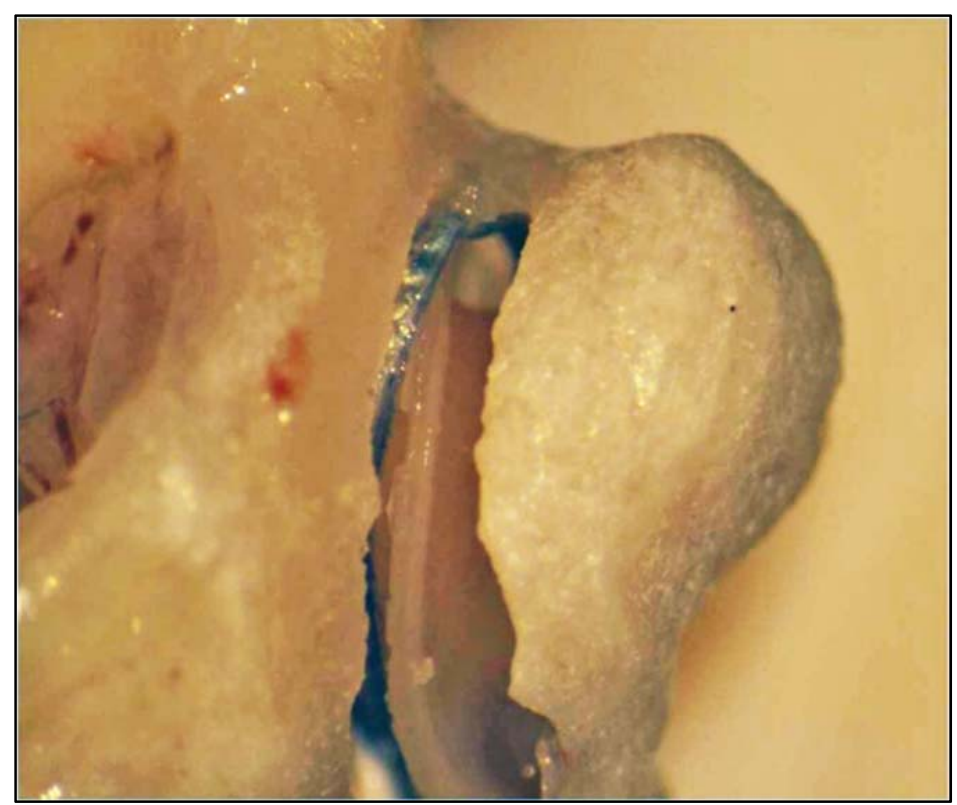

Fig. 4.4: A harvested cat cochlea after receiving a PC implant. The array can be seen jumping the scala media. 
The in-plane rigidity of the flat PET backing caused the array to flex towards the SV and advance through the scala media. Despite crossing over the membrane, insertions of the PC arrays were achieved to a depth of over $8 \mathrm{~mm}$, with all thirty-two electrode sites entering the cochlea. To the best of our knowledge, these arrays achieved the deepest insertion depth of any existing cat cochlear electrode array. However, insertion yields were poor as the PET backings were prone to delaminate from the array during insertion. As an alternative to the glued on PET tubes, monolithic ringed-channels were integrated into parylene arrays, which were pre-curved (wafer-level) by residually stressed parylene layers. These arrays were developed for the guinea pig animal model as the smaller animals were easier to access and more affordable.

Ring-backed parylene arrays with a $2 \mathrm{~mm}$ radius of curvature (MC) were implanted into guinea pig cochleae. Previous attempts to use the AOS technique were unsuccessful, because it was difficult to direct the arrays off of the stylet wire and through the cochleostomy. The angle of access to the cochleostomy is much more restrictive in the guinea pig versus the cat. As such, plated-nickel stylet wires of graded stiffness were fabricated for the MC experiments. Instead of advancing the arrays off of the stylet, both the wire and the array were advanced into the cochlea. These stylet wires were able to advance the MC arrays up to $6.3 \mathrm{~mm}$ into the guinea pig cochlea (Fig. 4.5, Fig. 4.6,). Like the PC arrays they crossed through the scala media. Without the ability to remove the straight wires from the arrays, the devices were not free to hug the modiolus and so the curling feature of the arrays could not be assessed. Future testing in cat animal models will have to be conducted to determine whether the arrays are as capable of hugging the modiolus in in-vivo experiments as they were in hugging the modiolus of in-vitro molds. Although the curvature capabilities could not be assessed, the ability to place a stylet-wire inside the rings and insert the entire assembly into the cochlea without tearing the rings or substrate, showed the arrays were much more robust than their silicon predecessors. It could be argued that the nickel-plated stylet in some way enhanced the robustness, however experiments implanting straight, ring-backed, arrays without the use of a stylet were still quite robust. 


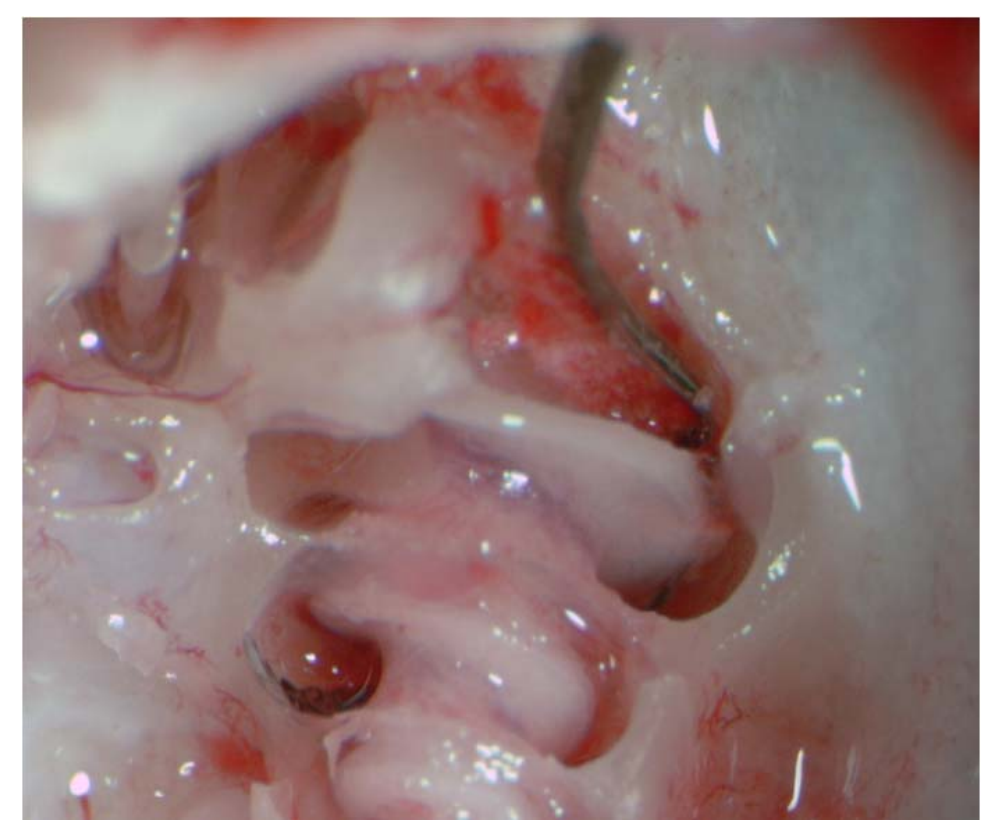

Fig. 4.5: A pre-curved and ring-backed (MC) array inserted $6.3 \mathrm{~mm}$ into GP cochlea with the aid of a graded-stiffness stylet wire. The array can be seen to cross over the scala media.

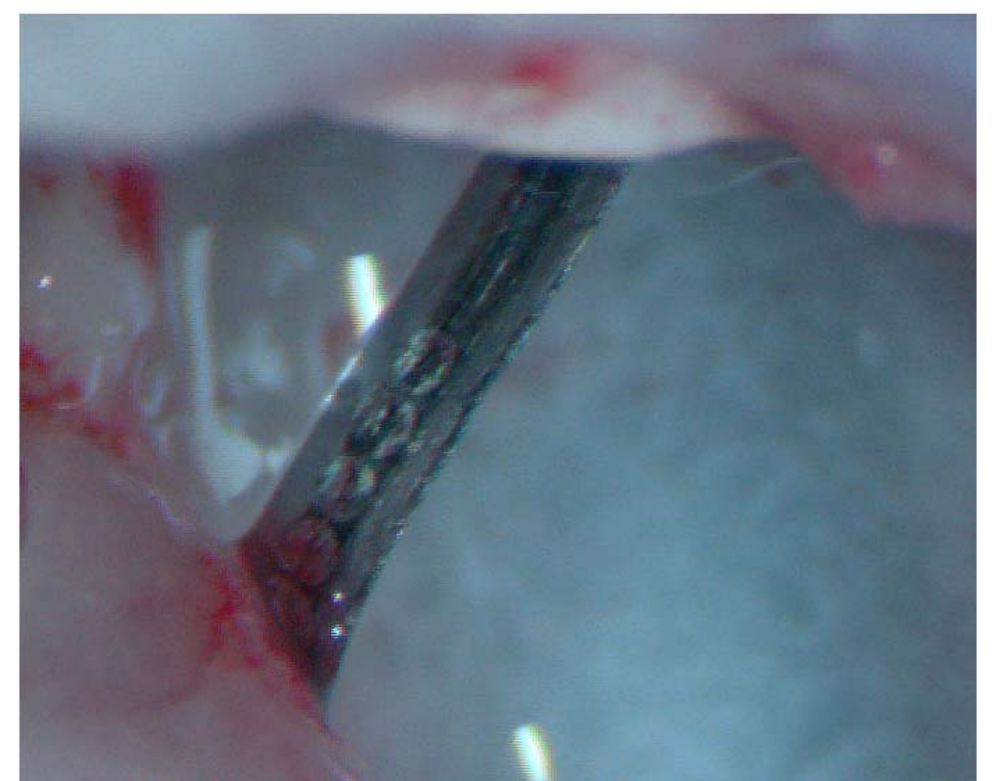

Fig. 4.6: A uniformly stiff, ring-backed (MC) array going through the middle ear space and into the cochleostomy.

Straight, ring-backed, parylene arrays of uniform stiffness (US) were implanted into guinea pig cochleae using direct placement with a pair of surgical tweezers. These arrays were excessively flexible and tended to bend outside the cochlea during insertions. 
The furthest insertion depths achieved were approximately $3 \mathrm{~mm}$ into the cochlea (Fig. 4.7).

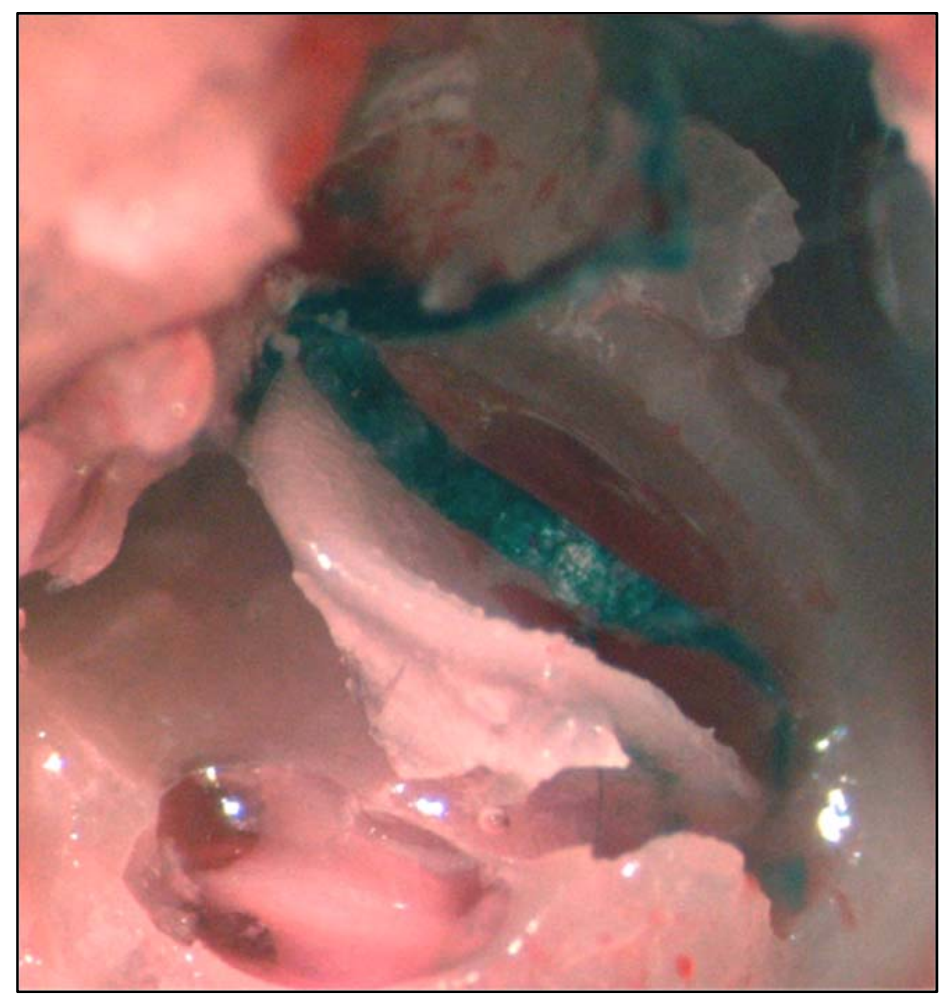

Fig. 4.7: A uniformly stiff, ring-backed (US) guinea pig array entering cochlea. Array did not progress further than $4 \mathrm{~mm}$, as shown above.

Several angles of insertions were tested including one that entailed inserting the array backwards (sites facing back wall of cochlea) and rotating it forward (sites facing the modiolus) once the shank made it past the first turn. All angles resulted in the arrays either buckling outside the cochlea or folding back on themselves 3-4 mm inside the cochlea. To eliminate this issue, ring-backed, graded-stiffness, parylene arrays (GS) were fabricated with graded stiffness built into their flexible substrates and soft ringedbackings.

Straight arrays of graded stiffness (GS) were inserted into a guinea pig cadaver cochlea to assess their performance. The GS arrays achieved insertion depths of up to 6.5 $\mathrm{mm}$. Moreover, they did not crossover the scala media and were positioned entirely within the scala tympani (Fig. 4.8). 


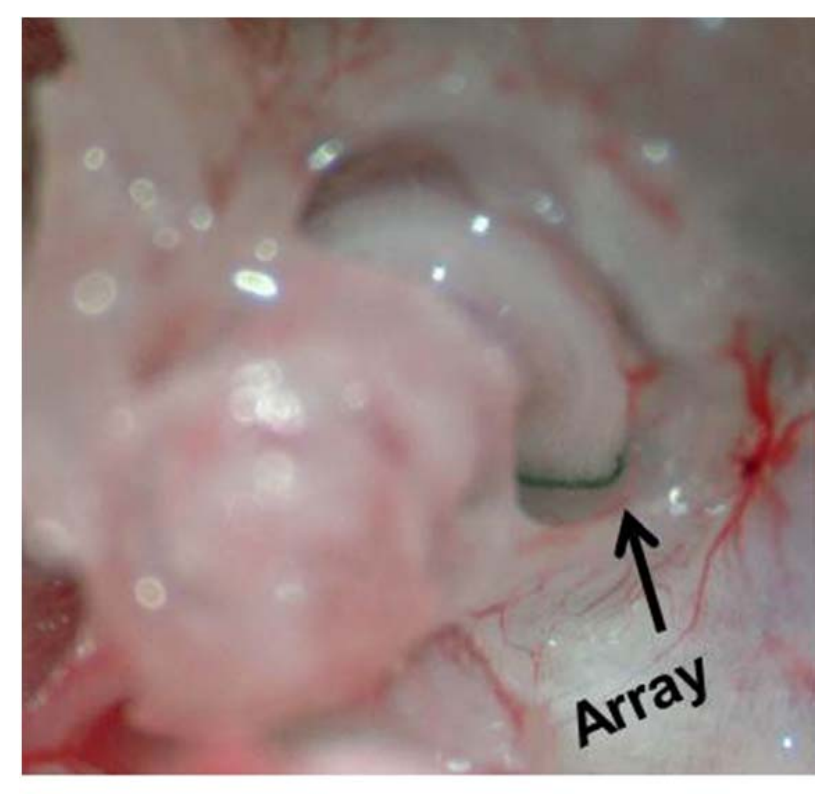

Fig. 4.8: A graded-stiffness parylene (GS) array going around first turn of guinea pig cochlea. The array was housed entirely within the scala tympani.

A comparison with the insertion depths achieved for a commercial implant reveals that the graded stiffness (GS) arrays achieve nearly $40 \%$ deeper insertion depths into the scala tympani, with twenty versus five electrode sites available for stimulation. An examination of the extracted arrays show that bending was confined to the transition point where the array changed from one stiffness level to the next (Fig. 4.9).

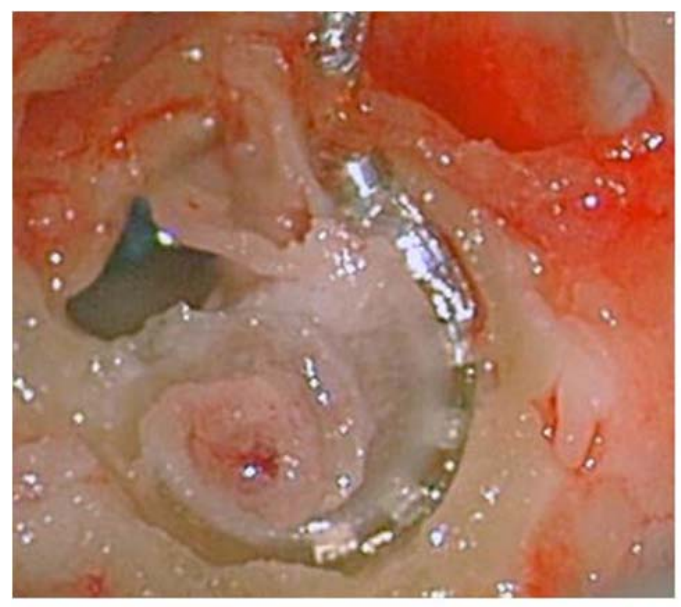

(a)

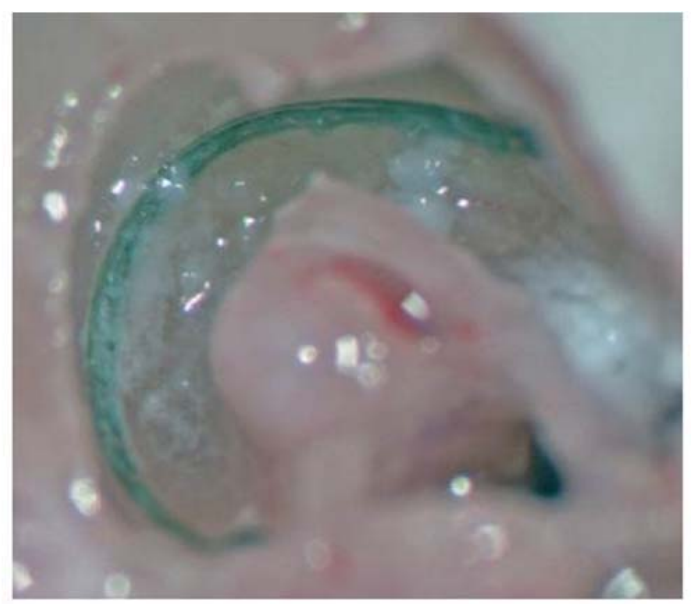

(b)

Fig. 4.9: Cochlear America's animal array inserted into a guinea pig cochlea (a) and (b) a ring-backed, graded-stiffness (GS) parylene electrode array inserted similarly. 
Future work will be done to determine the optimum stiffness grading for advancing the arrays fully into the second turn of the cochlea as discussed in chapter 6 .

\subsection{Performance using Electrical Stimulation}

To see if the IrO sites on the parylene cochlear arrays were capable of eliciting frequency-dependent auditory responses at reasonable threshold levels, in-vivo stimulation experiments were carried out. Two sets of experiments were performed. In

the first, the auditory nerve response to intracochlear stimulation was assessed with EABRs, characterizing the threshold levels of the IrO sites in comparison to commercial hand-assembled wire-bundle alternatives. A second set of experiments was used to show that the IrO sites can provoke neural activity in the IC through intracochlear stimulation. The threshold levels required to elicit a response in the IC were also determined.

Electrical stimulation was carried out using pre-curved, ring-backed parylene cochlear electrode arrays that were housed with a nickel wire of graded stiffness (MC). The arrays were implanted in the guinea pig cochlea and stimulated with monopolar site configurations. Brain responses were evoked in the auditory nerve with $25 \mu$ s- or 200 $\mu \mathrm{s}$-duration monophasic, alternating polarity pulses at $50 \mathrm{pps}$, as described in section 4.1.2. In total, EABRs were collected from six different guinea pigs using six different electrode arrays. The EABR responses varied over the implants depending on the state of the electrode arrays after implantation and the state of the animals at the time of recording. For pulse widths of $200 \mu \mathrm{s}$, the minimum thresholds recorded were near 30 $\mu \mathrm{A}$ (Fig. 4.10). For $25 \mu$ s pulse widths, minimum thresholds were $125 \mu \mathrm{A}$ (

Fig. 4.11). 


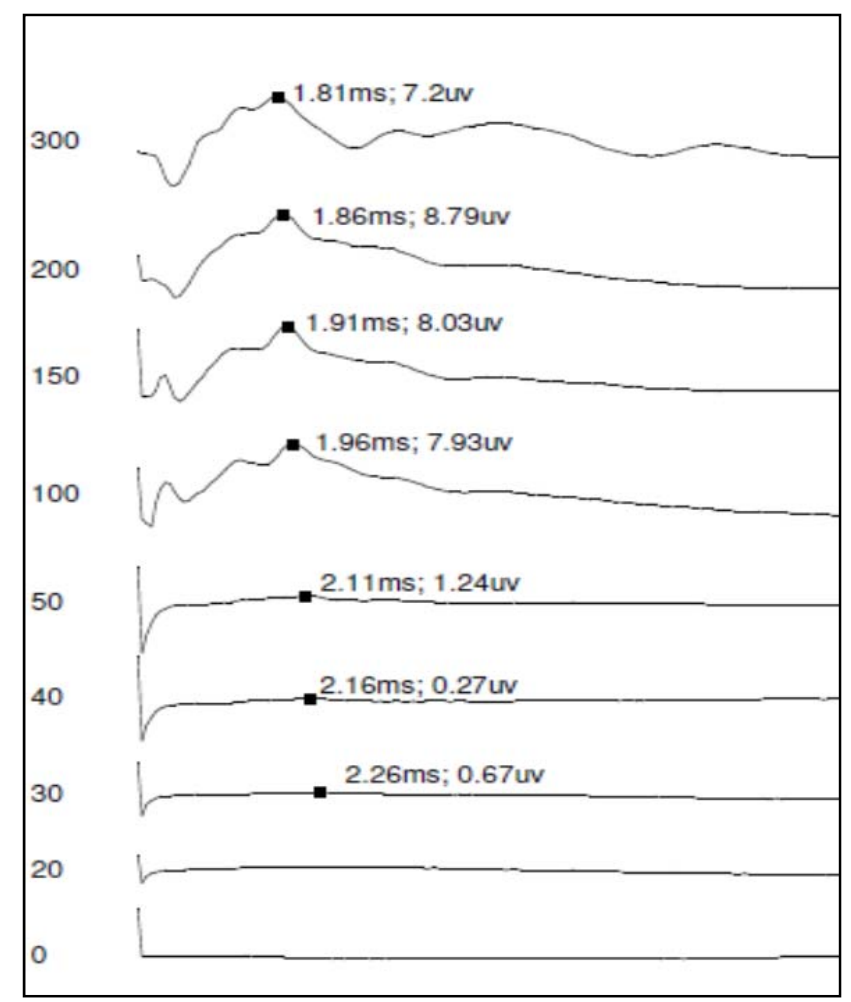

Fig. 4.10: Sample of neural activity recorded from EABRs in response to intracochlear stimulation of site number 21 using monophasic stimulus pulses with $200 \mu$ s phase durations.

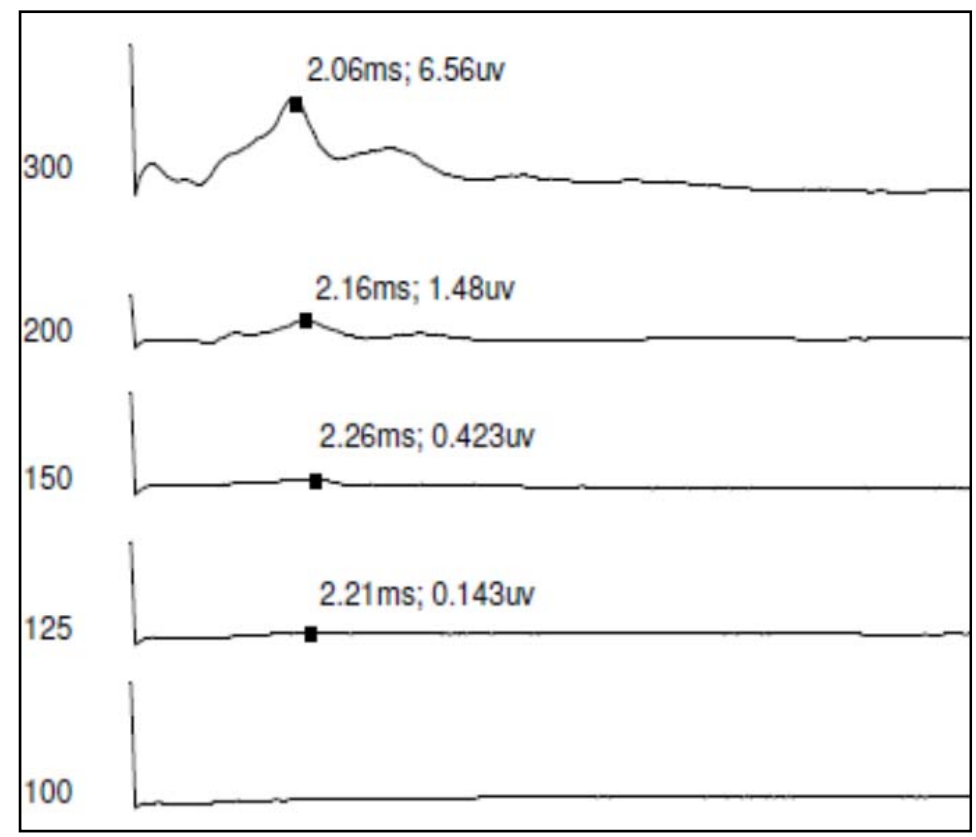

Fig. 4.11: Sample of neural activity recorded from EABRs in response to intracochlear stimulation of site number 16 using stimulus pulses with $25 \mu \mathrm{s}$ phase durations. 
The highest threshold values recorded for any array were $875 \mu \mathrm{A}$. This high threshold value corresponded to an array whose sites were severely fractured by repeated insertions. The impedance values for the sites that corresponded to these high threshold levels were well over $100 \mathrm{~K} \Omega$. In general, the thresholds of the EABRs collected from the thin-film arrays were comparable and in some cases better than what is typical of hand-assembled commercial arrays (Fig. 4.12).

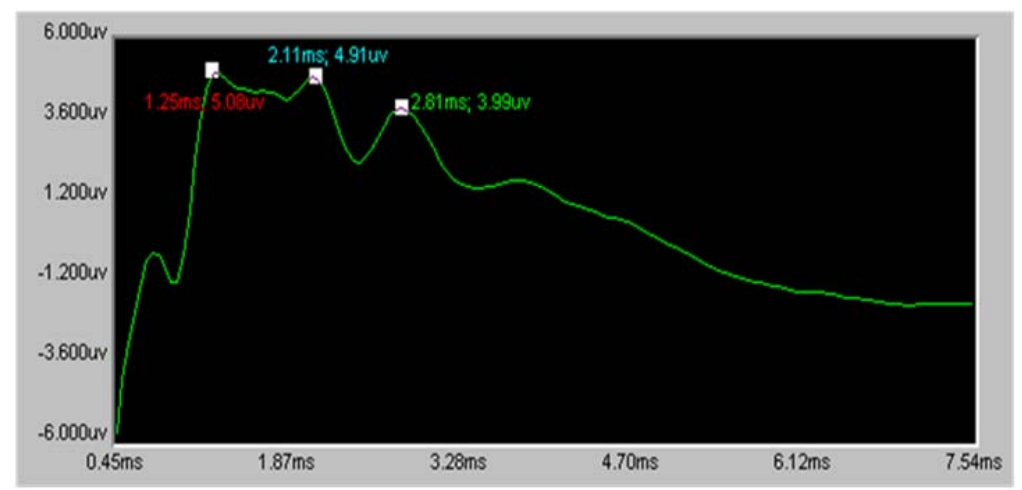

(a)

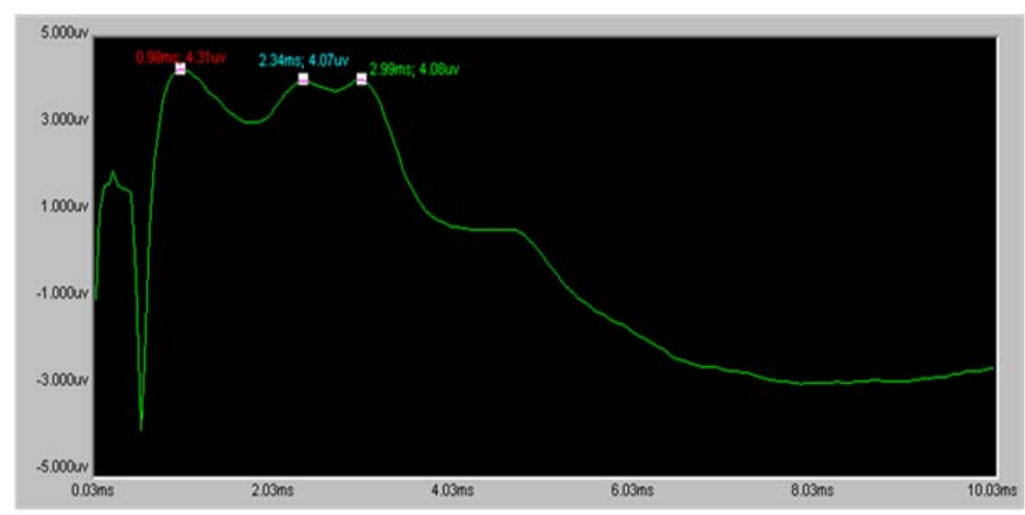

(b)

Fig. 4.12: Electrically evoked auditory nerve responses to stimulation with (a) Cochlear America's electrode array and (b) parylene electrode array. For $200 \mu \mathrm{A}$ pulses with monophasic, alternating polarity durations of $25 \mu \mathrm{s}$.

Experiments that involved intracochlear stimulation and measured responses from the IC used stimulus pulses with biphasic durations of $200 \mu$ s, and a stimulus rate of 4pps. PeriStimulus Time Histograms (PSTH) were generated to observe neuronal spikes that repeat in time in response to pulsatile stimulation of the intracochlear electrode array. The delay between biphasic pulses is $250 \mathrm{~ms}$, but for readability the histogram is cut-off at $200 \mathrm{~ms}$ as neural firing occurs within the first $100 \mathrm{~ms}$ after intracochlear stimulation. It should be noted that the spike seen within the first $10 \mathrm{~ms}$ is stimulus artifact and should 
not be considered a relevant response to intracochlear stimulation. Peaks in the histogram beyond the $10 \mathrm{~ms}$ bin indicate repetitive neural spikes in response to introcochlear stimulation. A noise floor is present throughout all recording channels, but is higher on some rather than others. This noise floor should also be neglected when viewing the histograms.

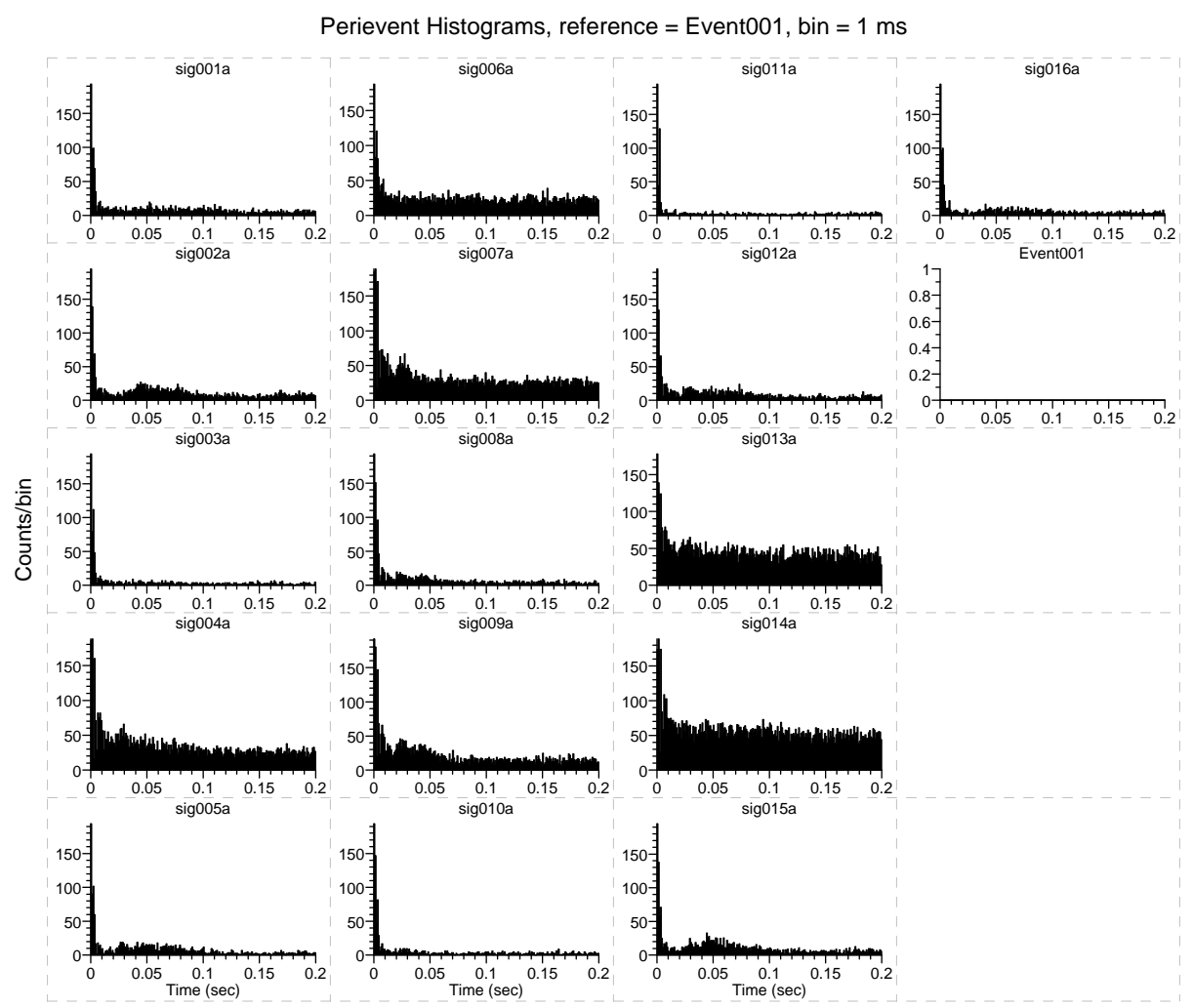

Fig. 4.13: Spike count versus the time following the onset of stimulation (in increments of $200 \mathrm{~ms}$ ). Neural firings in response to stimulation was picked up on recording sites 1-16 of a 16-site Neuronexus recording probe in the guinea pig IC in response to intracochlear stimulation of site number $21(50 \mu \mathrm{A})$. Activity is present on recording channels $2,5,8,9,12$ and 15 .

The thin-film sites were able to successfully initiate a response in the IC to intracochlear stimulation with thresholds as low as $50 \mu \mathrm{A}$ (Fig. 4.13). The neural response to monopolar stimulation above threshold $(100 \mu \mathrm{A})$ for the thin-film sites was quite similar to that of hand-assembled commercial arrays in that the monopolar sites activated a broad area of the IC and activity was picked up on several recording sites. The IC responded as expected to increased current amplitudes, with greater amplitudes resulting in increased neural activity and broader current spread (Fig. 4.14). 


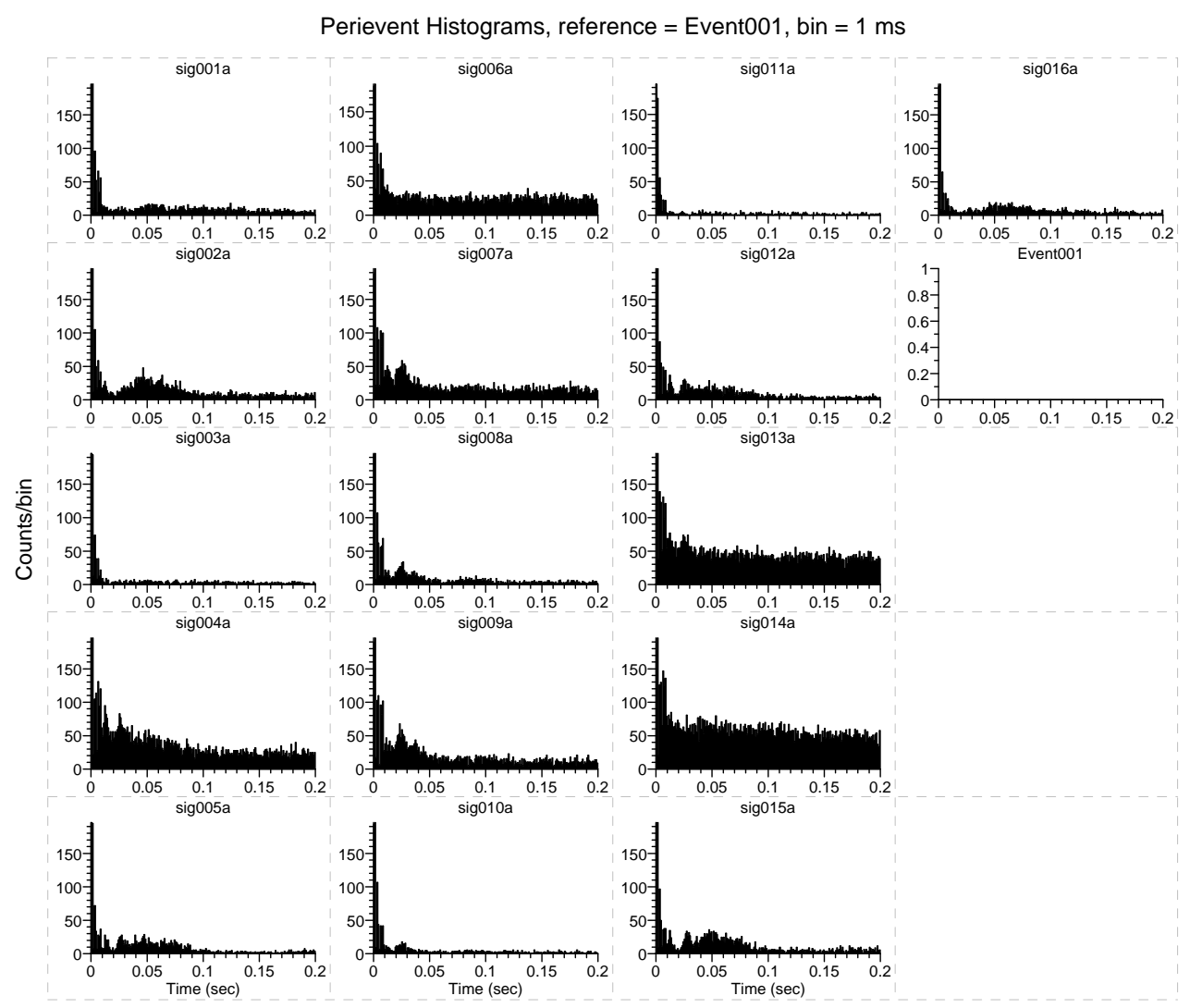

Fig. 4.14: Spike count versus the time following the onset of stimulation (in increments of $200 \mathrm{~ms}$ ). Neural firings in response to stimulation was picked up on recording sites 1-16 of a 16-site Neuronexus recording probe in the guinea pig IC in response to intracochlear stimulation of site number $21(100 \mu \mathrm{A})$. Activity is present on recording sites 2, 5, 7, 8, 9, 10, 12, 15 and 16 .

\subsection{In-Vivo Summary}

Two cat implants and twelve guinea pig implants were carried out using the parylene electrode arrays. PET-backed (PC) arrays of uniform stiffness achieved full insertion into the cat cochlea, achieving depths greater than any animal implant to date. Precurved and uniformly stiff arrays (MC) for the guinea pig model also achieved full insertions with the aid of a flexible stylet wire. To prevent the arrays from cutting through the scala media, parylene arrays of graded stiffness (GS) and high flexibility inplane were fabricated. The graded-stiffness implants did not cut through the scala media and achieved insertions depth of up to $6.5 \mathrm{~mm}$. Future work in the area of array mechanics will focus on increasing the overall stiffness of the graded (GS) arrays while maintaining the stiffness gradient. Thin-film IrO sites evoked responses in the auditory 
nerve at very low threshold levels of $30 \mu \mathrm{A}$. Similar threshold levels occurred for evoking responses in the IC. Monopolar characteristics were similar to those of handassembled commercial arrays, and there was a proportional relationship between current amplitude and neural activity. Having assessed the capacity of the thin-film sites to neural responses in the auditory nerve and the Inferior Colliculus, future work will focus on assessing the multipolar performance of the sites. Having sites of similar diameter, monopolar stimulation across the thin-film array is quite similar to hand-assembled implants. However, with a decreased center-to-center spacing of $250 \mu \mathrm{m}$ compared to a minimum center-to-center spacing of $450 \mu \mathrm{m}$ for the commercial hand-assembled arrays, multipolar configurations have the potential to perform much better than hand-assembled arrays when it comes to frequency selectivity. This has been demonstrated in the past by the thin-film silicon cochlear electrode arrays [1].

\subsection{References}

[1] Bhatti, P., "A high-density thin-film electrode array for a cochlear prosthesis," in Electrical Engineering and Computer Science, Ph.D. Ann Arbor: The University of Michigan, 2006 


\section{Chapter 5}

\section{ACTIVE ELECTRODE ARRAY}

A hybrid ASIC will be integrated into the passive array. The cochlear ASIC will increase the functionality of the high density cochlear electrode array in three ways: (1)

It will reduce the voltage drops from the receiver of the prosthesis to the electrode array by decreasing the connecting cable from 128 tightly pitched lead lines to just 9 lines, which can be made much wider. (2) It will reduce the stiffness and increase the robustness of the connecting cable by reducing the number of leads going down it. (3) It will create an active array capable of handling a diverse range of site configurations, pulse train specifications, and current stimulating schemes.

The ASIC contains a four-channel pulse generator and has built-in functions for assessing the state of the high-density system. Some of these functions are diagnostics for verifying correct operation of the ASIC I/O and pulse generation circuitry, while others are geared toward monitoring and altering the state of the electrode array by measuring site impedances, grounding sites between pulses, and activating sites post hybrid assembly. Although, its immediate use is in the research domain as a stand-alone assessment tool, the chip was also designed to interface with a wireless microcontroller specified elsewhere [1] as part of a fully implantable cochlear microsystem. As such, operation is controlled by a 16-bit command word, a handshaking routine is employed for timed chip-to-chip communication, and all operations are generated on-chip without using off the shelf components or external pulse generators.

In the past, a dual mode, 4-channel, 32-site ASIC was developed for a previous generation of the Michigan active cochlear electrode array [2]. The $2.4 \mathrm{~mm} \times 2.9 \mathrm{~mm}$ ASIC was integrated with the thin-film array, and operated from a $\pm 2.5 \mathrm{~V}$ dual power supply. It was capable of generating biphasic pulses at up to $500 \mu \mathrm{A}$ with a power consumption of $2.5 \mathrm{~mW}$. The ASIC operated in stimulation mode, sending biphasic pulses to the sites, and in extended mode while recording from position sensors and 
electrode sites and performing system functions (Fig. 5.1). Each channel of the ASIC was connected to a bank of eight sites, and each bank was tied to one of four current drivers [3]. The drivers were controlled from a single DAC. Depending on which channel was selected, the output of the DAC would be sampled and held on a capacitor specific to that channel. With this scheme, up to four sites could be simultaneously active, but only one site from each bank could be active at a time (one-of-eight selections). This greatly limited the ways sites across the array could be used in bipolar or tripolar site configurations. Additionally, the sample-and-hold capacitor posed system stability issues since the rate at which stored charge on the capacitor decays is dependent on in-vitro conditions such as body temperature.

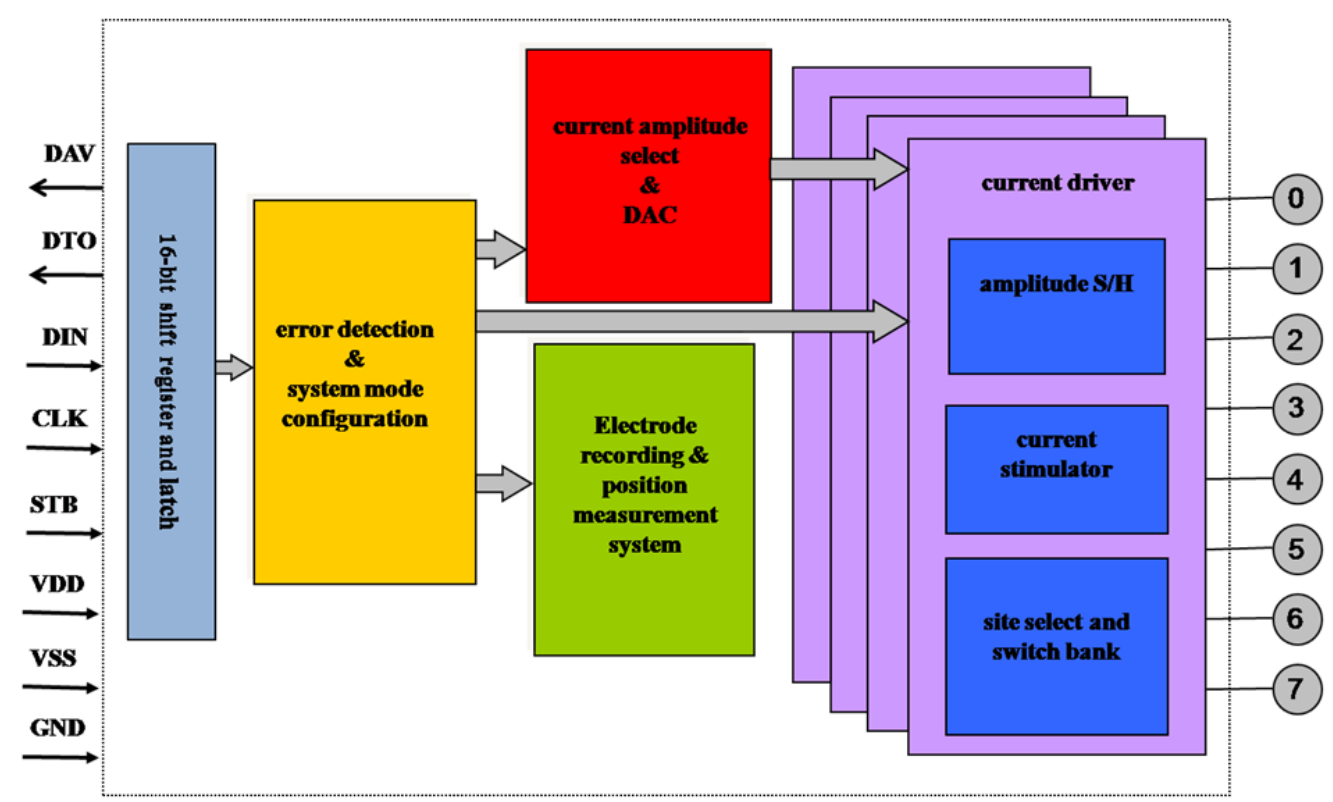

Fig. 5.1: Previous generation ASIC [2]

For the next-generation cochlear array presented in this work, the ASIC has been revisited to increase its site-selection capabilities and system stability. Each of the four channels now has a dedicated DAC, and the sites are distributed among these channels in such as way that bipolar and tripolar configurations can be used with a broad range of site separations.

In this chapter an overview will be given of previous cochlear ASIC designs, followed by a detailed explanation of the considerations for the next-generation ASIC. 
Next, an operational view of the new ASIC will be given along with a presentation of measured chip performance.

\subsection{System Organization and Specifications}

The WIMS cochlear prosthetic system consists of a wireless microprocessor, internal pulse-generating (IPG) ASIC, and high-density thin-film cochlear electrode array (Fig. 5.2).

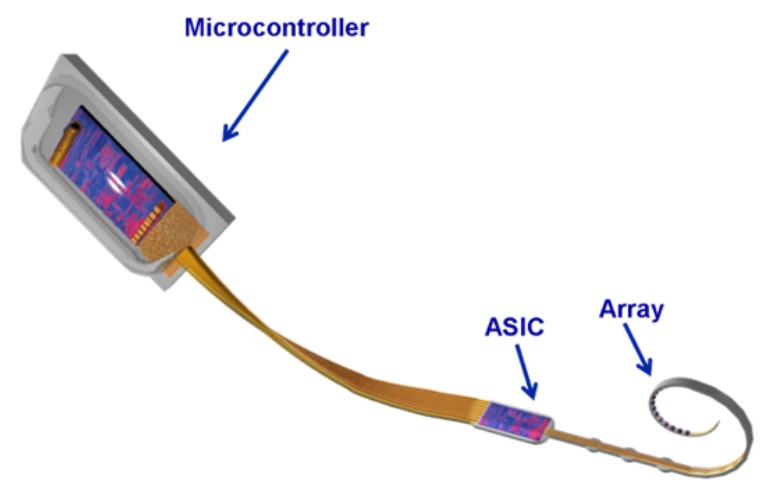

Fig. 5.2: Thin-film cochlear prosthetic system.

The microprocessor has wireless capabilities for reiceving power and input from an external microphone as well as a digital signal processing core that uses the CIS algorithm to generate 16-bit stimulation commands for the IPG. This processing unit is connected to the cochlear ASIC through an 8-lead command cable. The ASIC decodes commands sent by the wireless microprocessor into stimulus pulses for the designated sites. The high-density array is bonded to the ASIC using a monolithically integrated cable [2]. The work of this thesis was focused on improving the IPG ASIC design, architecture, and performance. In particular, considerations were given to expanding the possible set of stimulating configurations and to equipping the ASIC with functions for performing system-level diagnostics, electrode site modifications, and site characterization in-vivo. 


\subsubsection{Stimulation Configurations}

In the next-generation ASIC, multipolar site configurations and simultaneous as well as non-simultaneous stimulation features were expanded to further push and assess the limits of frequency selectivity in a high-density array.

It has been shown that the number of electrodes (i.e., distance) separating adjacent sites in a multipolar (bipolar or tripolar) configuration plays a significant role in the perceived frequency selectivity [4]. The closer the electrodes are together, the greater the selectivity. However, in the previous ASIC, the distance between site pairs or triplets could not be selected independent of array location. That is, the sites within each channel bank could not be configured in bipolar or tripolar configurations. Further, only electrode pairs $(25,24),(17,16)$ and $(9,8)$ could be used for bipolar stimulation between adjacent electrodes $(\mathrm{BP}+0)$. This restriction limits the frequencies that can be most selectively activated to those situated just above these electrode pairs. It was impossible to do a balanced tripolar configuration with three adjacent electrodes $(\mathrm{TP}+0)$. To fully explore and make use of these high-density thin-film arrays, it is advantageous to remove this restriction in site selection. One way to expand functionality is to redistribute the channels across the array using a design first presented by Gingerich and Wise [5]. Instead of assigning eight consecutive sites to a particular channel, the array interface to the ASIC can be reorganized by distributing the channels all across the array (Fig. 5.3).

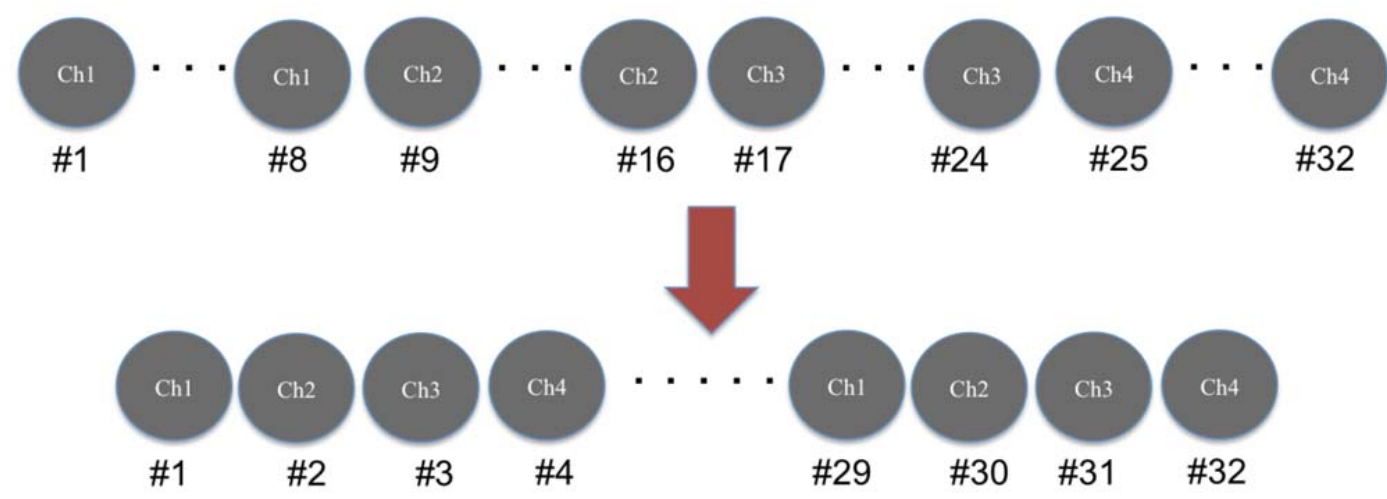

Fig. 5.3: An ASIC architecture where each channel is assigned to eight consecutive sites versus a distributed assignment where the channels are distributed in an alternating fashion among the sites. 
This allows for $\mathrm{BP}+0$ and $\mathrm{TP}+0$ configurations on any two or three adjacent sites across the array. Additionally, any site across the array can be stimulated in a $\mathrm{BP}+1 / \mathrm{BP}+2$ or $\mathrm{TP}+1 / \mathrm{TP}+2$ configuration. In general, provided there are sites within range, any electrode can be configured in a $\mathrm{BP}+\mathrm{N}$ or $\mathrm{TP}+\mathrm{N}$ configuration as long as $\mathrm{N}+1$ is not a multiple of 4 .

In addition to modifying the addressable site configurations, frequency selectivity can be further improved by varying the ratio of current contributed from each site in the multipolar configuration. Such a strategy has been used by Advanced Bionics to create over 150 "virtual" electrode channels from only 22 hard-wired site [6]. Each virtual site is created by steering the peak of the current spread profile such that it targets different cell groups for each different ratio of biphasic pulses (Fig. 5.4). The peak is steered by using varying amplitudes of Current Units $(\mathrm{CU})$ on pairs/triplets of sites, where a $\mathrm{CU}$ is the allowable increment of current amplitude for a given pulse generator.
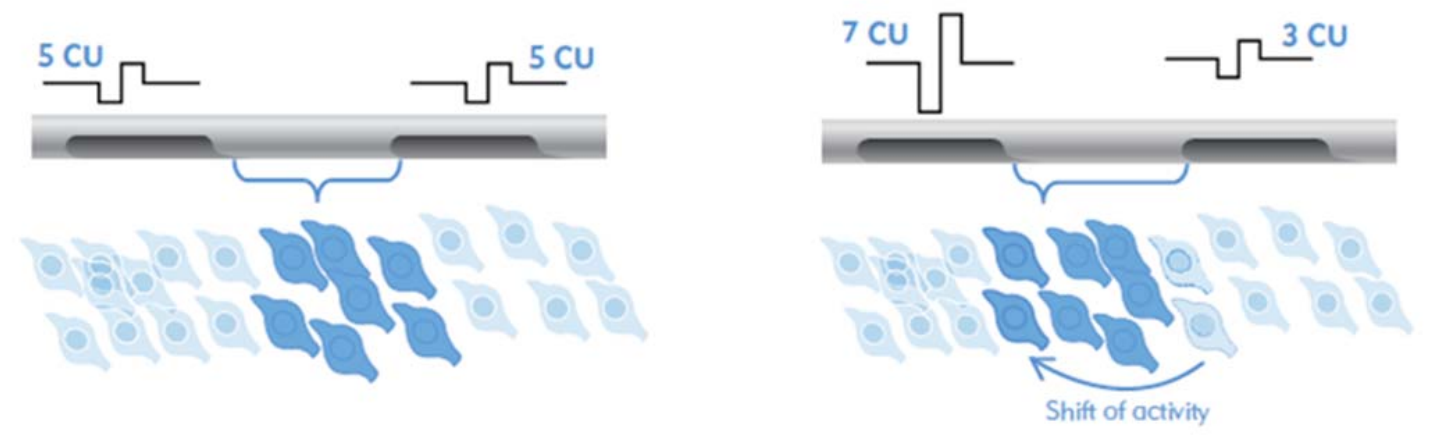

Fig. 5.4: The charge between two sites, measured in medical Current Units (CU) is distributed equally, with $5 \mathrm{CU}$ per site (A), and in a ratio of $7 / 3$, with $7 \mathrm{CU}$ on one site and $3 \mathrm{CU}$ on the other (B). Two different frequencies can be perceived for (A) and (B) since two distinct cell groups are stimulated as depicted by the darker coloring [6].

Using this approach, two hardwired sites (S1, S2) can be used to create three additional "virtual" sites (V2, V3, V4) by dividing the current into 80/20, 50/50 and 20/80 splits (Fig. 5.5). 


\section{$\begin{array}{lllll}100 / 0 & 80 / 20 & 50 / 50 & 20 / 80 & 0 / 100\end{array}$}

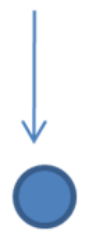

S1

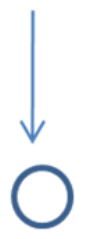

V2

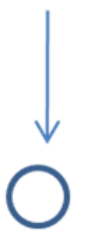

V3

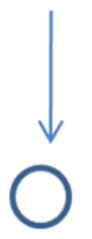

V4

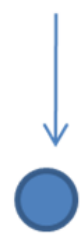

S5

Fig. 5.5: Two hardwired sites are used to create and additional three "virtual" sites by dividing the current across the sites in three different ratios.

It has been shown these "virtual" channels can lead to improved speech perception among cochlear implant users through their more selective activation of frequency sets $[7,8,9]$. With a high-density array having many more hard wired sites a greater number of virtual sites can be achieved. Additionally, with closer electrode site center-to-center spacing, the current spread of the virtual sites is reduced and, in turn, this could reduce channel interactions resulting in increased pitched descrimination. The new ASIC has been updated with a dedicated-DAC architecture that can be used to investigate the benefits of virtual channels in a high-density cochlear electrode array.

Simultaneous stimulation allows multiple frequency components of sound to be presented to the cochlear implant user at once. This type of stimulation is closer to the way normal hearing occurs. However, in past studies, it was found that simultaneous stimulation would tend to reduce frequency selectivity because the current spread of simultaneously active sites was so large that overlapping would occur and lead to frequency smearing [10]. Using the more frequency-selective configurations discussed above, it may be possible to reduce the spread of current to such a degree that effects of frequency smearing can be eliminated. Although a varied set of bipolar and tripolar configurations can be achieved using only three channels, a fourth channel was added to allow for simultaneous stimulation of bipolar configurations. 


\subsubsection{Site Manipulation and System Verification}

Once the hybrid ASIC is connected to the electrode array, it is up to the ASIC to prepare the array for stimulation as well as to monitor the array sites in-vitro or in-vivo. These functions include activating the electrode sites, measuring their impedance in operation, and grounding them after stimulation to remove any build-up of charge.

In the passive electrode array, the IrO sites are activated using cyclic voltammetry as discussed in Chapter 2 . The same setup can be used with active arrays by using a built-in activation function in which all of the sites are activated at the same time. To determine whether the electrode sites are functional and monitor the back voltage compliance, the ASIC is designed with capabilities for recording the impedance of each site in-vivo and in-vitro. Although, biphasic current pulses are used for stimulation, small charge imbalances between the anodic and cathodic pulses can build up over time. To ensure this build up does not approach the water window, the sites should be grounded periodically.

In addition to accessing the electrode array of the active system, it is also desirable to assess the performance of the ASIC itself. The ASIC was designed with capabilities for remotely verifying correct I/O functionality and characterizing the DAC performance.

\subsection{System Operation}

The ASIC is driven through a 9-wire Serial Peripheral Interface (SPI) bus (DIN, DAV, DTO, STB, CLK, RST, VDD, VSS, and GND) and operated from a 16-bit command word (Fig. 5.6). There is one additional I/O line, RST, which was added to this ASIC for simplicity and that was not included in the previous-generation ASIC.

The power supplies VDD and VSS are $\pm 2.5 \mathrm{~V}$; GND is referenced to animal ground. The SPI protocol begins with the microcontroller sending data to the ASIC on

the data line (DIN). This data is serially shifted into a 16-bit shift register on the rising edge of the clock (CLK) provided by the microcontroller. After all 16 bits are shifted into the register, they are latched by a strobe signal (STB) into a 16-bit latch. From here 
the bits are passed to a error detection module that runs an even parity check. Bit 9 of the command word is used to keep the parity of valid commands even. The results of error detection are sent over the data valid output line (DAV) and used to control hand-shaking between the WIMS microcontroller and the cochlear ASIC.

\section{Close up}

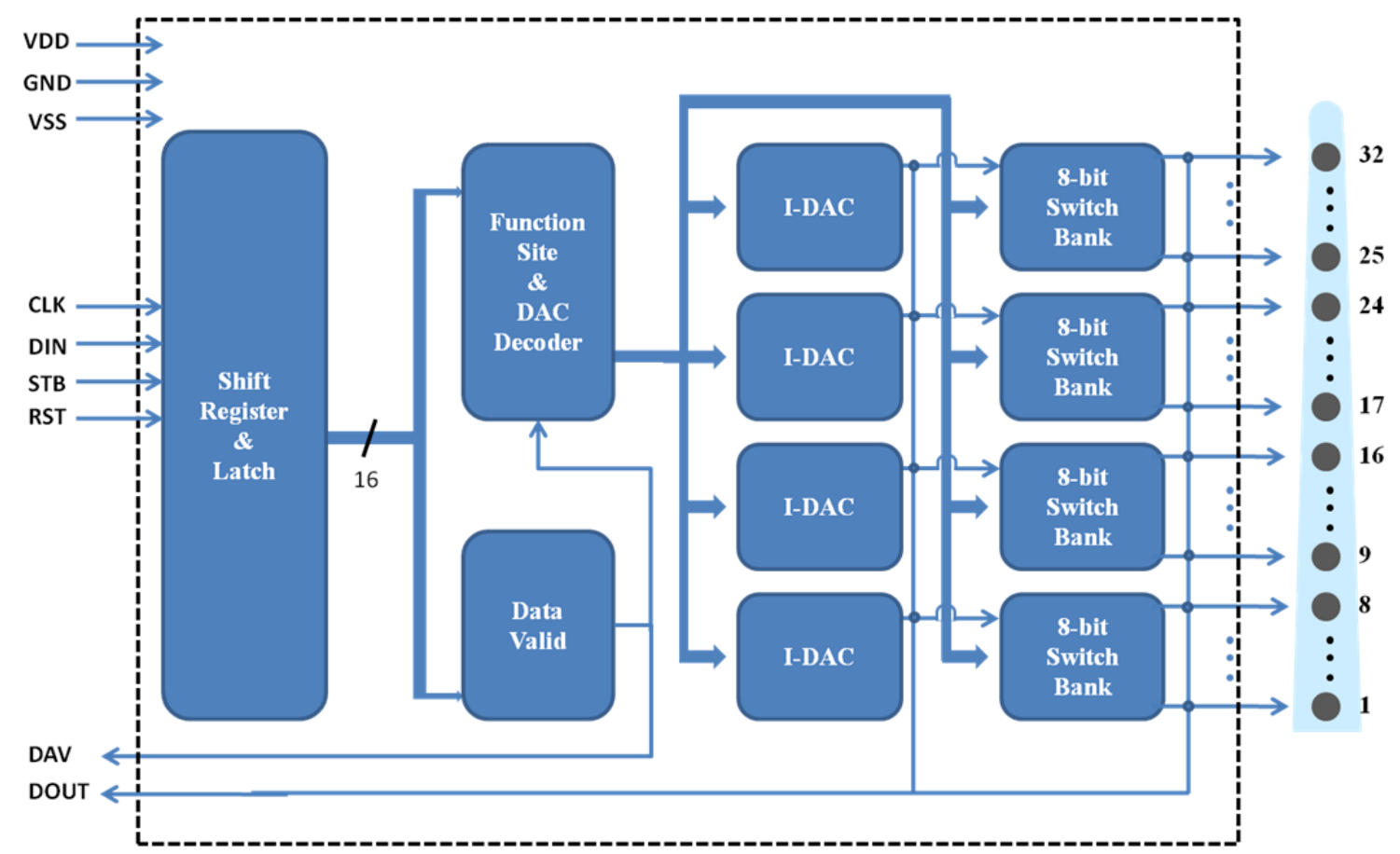

Fig. 5.6: System-level view of the cochlear ASIC

Assuming the data is valid, the command word is translated by a digital decoder block into a series of flag bits that control the mode, function, and current stimulation operation. The 16-bit command word is broken up into segments for controlling the ASIC mode (stimulation or extended), site selection, channel selection, parity, extended mode functions and current amplitude (Fig. 5.7). 


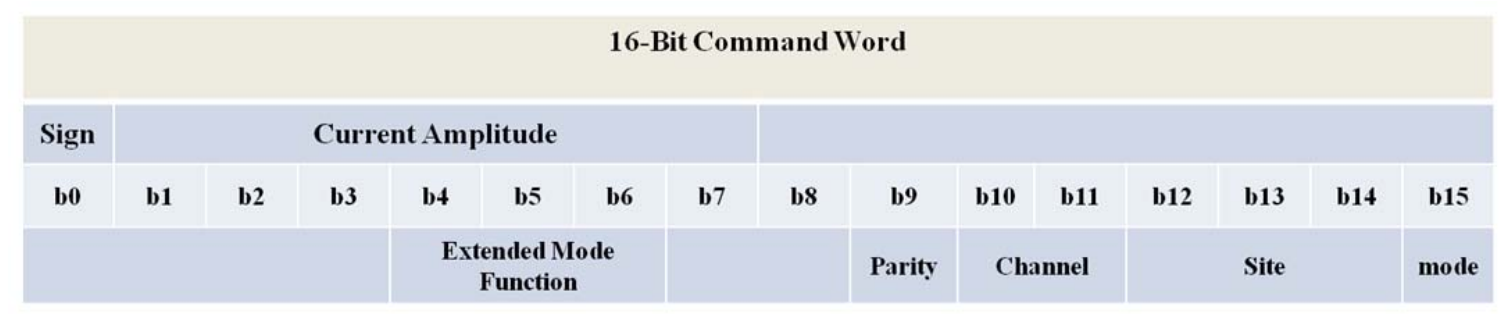

Fig. 5.7: 16-bit command word and bit designation.

A set of decoders is used to select an active I-DAC channel and control an 8-bit bank of switches that gate the I-DAC channels to the electrode array sites. Bit 15 of the command word is used to engage the ASIC in either stimulation (0) or extended mode (1) operation. In stimulation mode, bits 10-11 select one of the four I-DACs and bits 0-7 select the current amplitude of that I-DAC, which ranges from $4 \mu \mathrm{A}$ to $500 \mu \mathrm{A}$ in $4 \mu \mathrm{A}$ increments. The current output is directed to one of eight-sites in the selected channel using bits 12-14 as encoders for site selection. In extended mode, bits 9-15 are used in the same manner as for stimulation, but bits 4-6 are used to choose between one of the five extended mode functions (site impedance recording, shift register test, site activation, DAC calibration, and site grounding).

When commands initiate an ASIC action, the ASIC continues in this action until the next command is received. To put the ASIC in an idle state, a zero amplitude command can be initiated in either stimulation or extended mode. In stimulation mode, a zero amplitude command is performed when bits b0-b7 all equal zero. In extended mode, a zero amplitude command is performed when bit 3 equals zero. The duration of stimulation and extended mode functions is controlled externally by timing the sequence of command word inputs. For example, to stimulate with a $500 \mu \mathrm{A}$ biphasic pulse width of $100 \mu \mathrm{s}, \mathrm{a}+500 \mu \mathrm{A}$ command on the selected channel must be followed $100 \mu \mathrm{s}$ later by a $-500 \mu \mathrm{A}$ command on that channel which is ended after another $100 \mu$ s delay by a zero amplitude command (Fig. 5.8). During this biphasic pulse, pulses can be generated on other channels by interleaving other commands. 


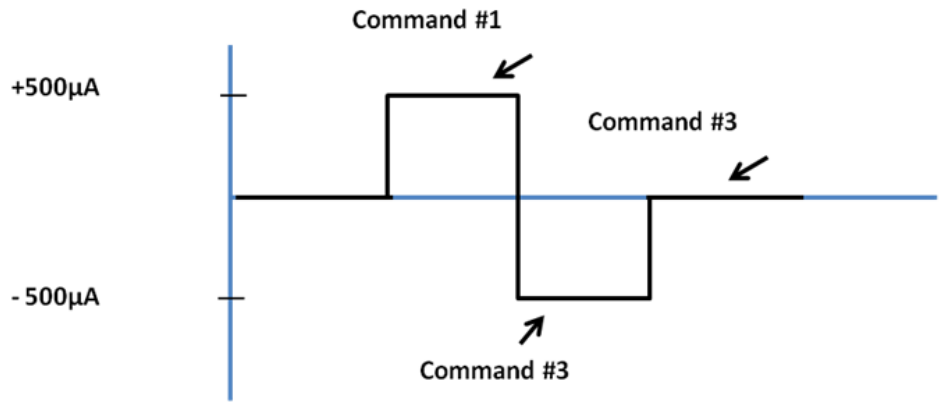

\begin{tabular}{|c|c|c|c|c|c|c|c|c|c|c|c|c|c|c|c|c|}
\hline \multirow[b]{2}{*}{ Command\# } & \multicolumn{16}{|c|}{$500 \mu$ A Biphasic Pulse Command Sequence } \\
\hline & b0 & b1 & b2 & b3 & b4 & b5 & b6 & b7 & b8 & b9 & b10 & b11 & b12 & b13 & b14 & b15 \\
\hline 1 & 1 & 1 & 1 & 1 & 1 & 1 & 1 & 1 & D & 1 & $\mathbf{0}$ & 1 & $\mathbf{0}$ & $\mathbf{0}$ & $\mathbf{0}$ & $\mathbf{0}$ \\
\hline 2 & 1 & 1 & 1 & 1 & 1 & 1 & 1 & 0 & D & 1 & $\mathbf{0}$ & 1 & 0 & 0 & 0 & 0 \\
\hline 3 & 0 & 0 & 0 & 0 & 0 & 0 & 0 & 0 & D & 1 & 0 & 1 & 0 & 0 & 0 & 0 \\
\hline
\end{tabular}

Fig. 5.8: Command sequence for a $500 \mu \mathrm{A}$ biphasic pulse. The pulse is generated with two stimulation commands followed by an extended-mode zero amplitude command.

Thus far, an overview of the system architecture has been presented. In the following sections a detailed look will be taken at each operational mode of the ASIC.

\subsection{I/O Verification}

During transmission of the command word from the WIMS microcontroller to the ASIC, it is possible that some bits may be corrupted. To verify that the proper command word is being read into the cochlear ASIC, a check for data validity and a shift register test command are available. The I/O section of the ASIC consists of a 16-bit shift register for serial input of a command word, a 16-bit latch to hold the command, and a data-valid block to validate the command parity (Fig. 5.9).

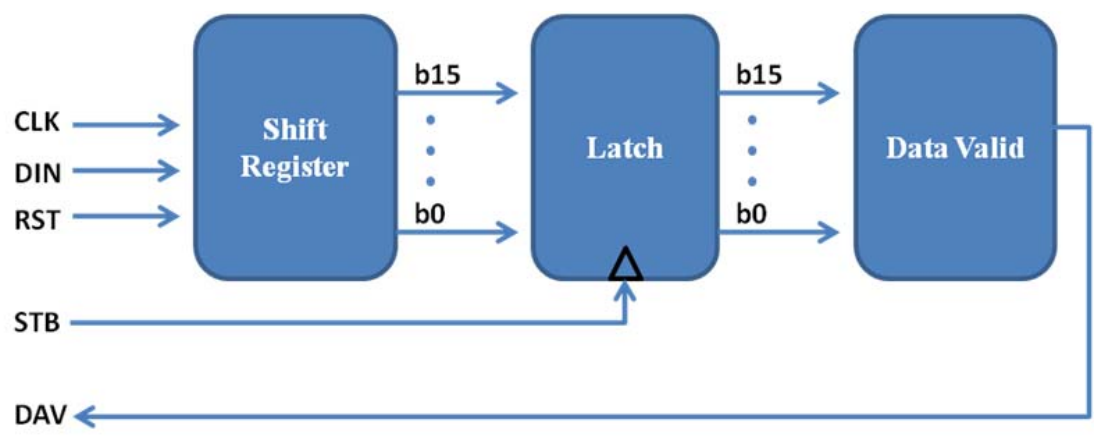

Fig. 5.9: I/O functional block diagram. 
Before any commands are read into the ASIC, RST is driven low to clear both the shift register and the latch and to reset the system. A command word is read into the ASIC on the positive edge of CLK in 16 cycles, and after the falling edge of the $16^{\text {th }}$ cycle, the STB line is pulsed high to latch the command and initiate a data-valid check. The data valid digital block runs an even parity check on the command word once it passes through the shift register and latch (Fig. 5.10).

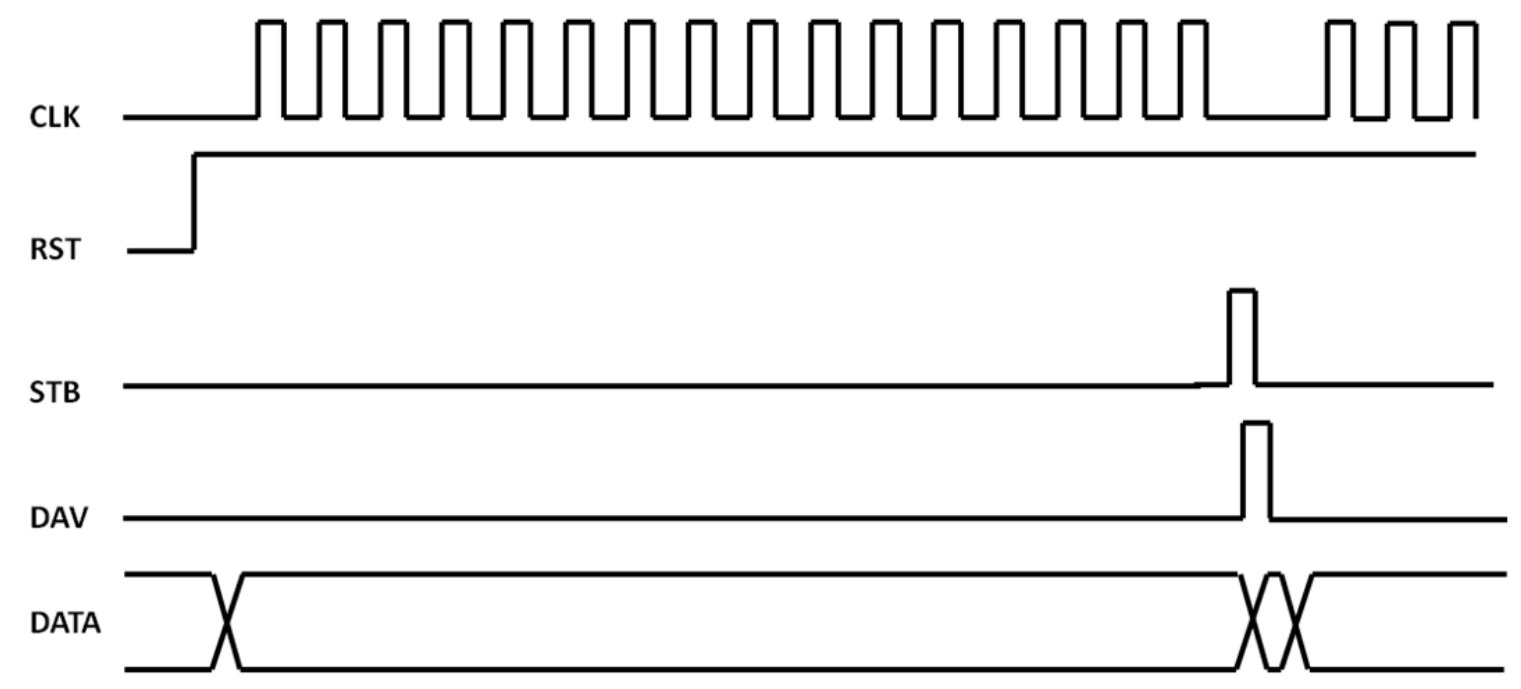

Fig. 5.10: I/O timing diagram.

If the command word has even parity, the ASIC runs the command. If DAV does not pulse high within two CLK periods, the command is assumed invalid and the microcontroller resends it. To avoid DAV from checking the command before the entire 16-bits are shifted in, it is set to zero whenever STB is equal to zero. Only after the strobe is set high can DAV go high, assuming the parity is even.

If the data is not valid, it is possible that the shift register itself is not working correctly. To verify correct operation of the shift register, a test can be run which connects bit 0 of the shift register to the DOUT line, so that the command bits can be read out in reverse order from the way they were shifted into the register. A shift register test operation is performed with the ASIC in extended mode by setting bits 4,5,6 to $1,0,0$ (Fig. 5.11). 


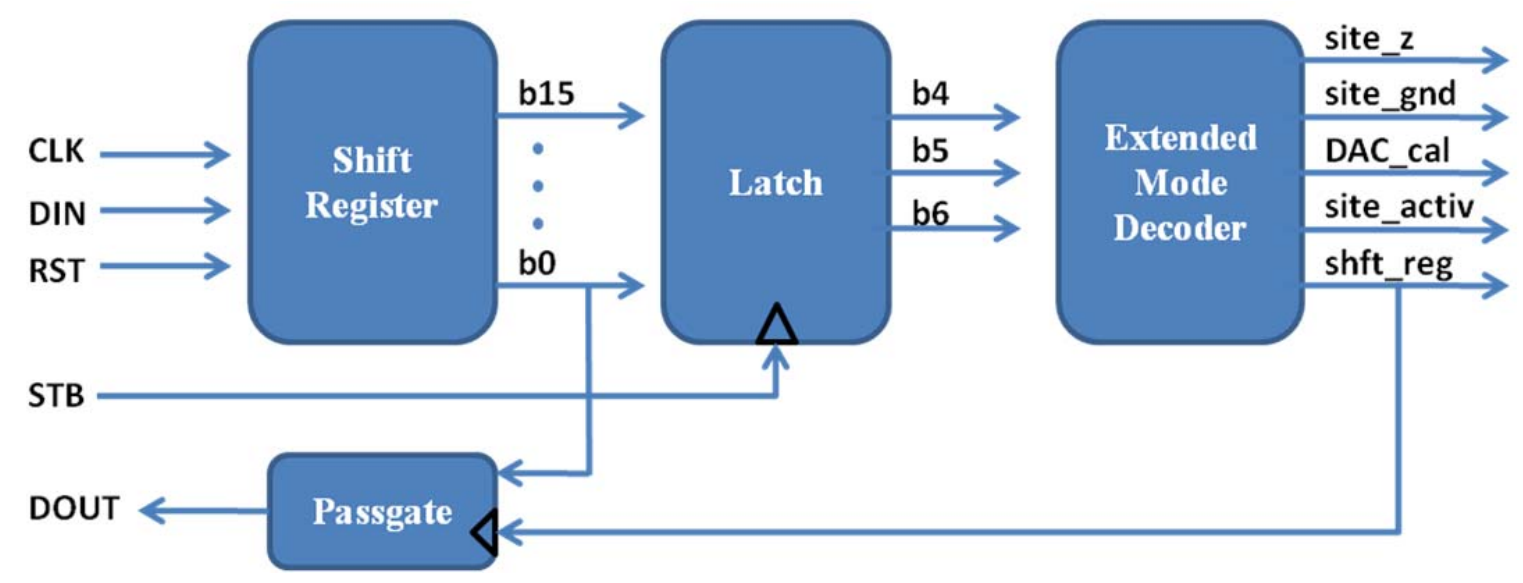

Fig. 5.11: Shift register test functional block.

The shift register test makes use of a pass-gate connected to the DOUT line. Control of this pass-gate is generated by decoding command bits b4-b6 into a shft_reg flag in extended mode. Although not depicted above, a shift register test also results in the I-DACs being set to zero amplitude to prevent unwanted stimulation and reduce power consumption.

\subsection{I/O Pulse Generation}

The cochlear ASIC has replaced the shared DAC architecture of the previous generation for a dedicated-DAC design where each channel of eight sites contains its own dedicated DAC. In this architecture, a stimulus pulse is generated by selecting one of the four I-DAC channels with bits b10-b11. These command bits are translated with a 2-to-4 decoder into an enable signal for the corresponding DAC latch. If a channel is not selected during a particular command, its latch is not updated and it maintains the state from the previous command. In this way, multiple channels can be driven at the same time for simultaneous stimulation of up to four sites (Fig. 5.12). 


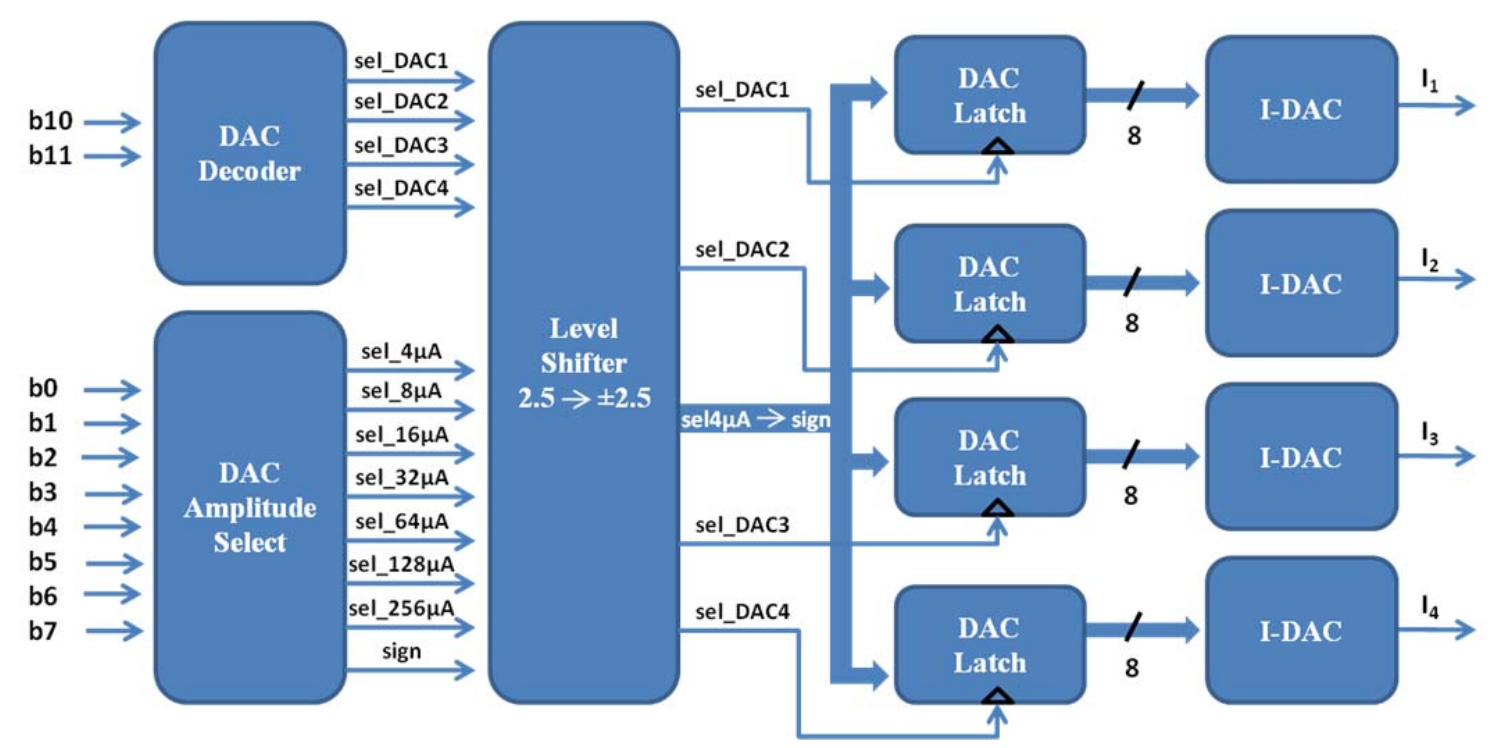

Fig. 5.12: Pathway of functional blocks used for channel selection.

The current amplitude and sign of the I-DAC are assigned with bits b0-b7. These bits go through the DAC amplitude select block where they are either passed through unchanged or converted to all zeros. Conversion to an all-zero state occurs when the ASIC is in extended mode and measuring site impedance. For this operation it is not necessary to have the I-DACs on, and they are shutoff to reduce static power dissipation.

To generate a biphasic pulse, the I-DAC and pass transistors of the switch bank need to sink to $-2.5 \mathrm{~V}$. However, $\pm 2.5 \mathrm{~V}$ is not needed to run the $\mathrm{I} / \mathrm{O}$, site multiplexing, latching and decoding functions across the ASIC. As such, power is conserved by operating these circuits blocks from a single $+2.5 \mathrm{~V}$ power supply and using level shifters to run current generation from $\pm 2.5 \mathrm{~V}$. The level shifters could be positioned right before the I-DAC and switch banks, but they are placed before the DAC latches to reduce the number of level shifters from thirty-six to twelve. Fewer level-shifters corresponds to a smaller footprint as well as less leakage current (reduced power consumption).

\subsubsection{The I-DAC}

Biphasic stimulus pulses are generated using the I-DACs. These circuits were designed to have output current stability over varying site/tissue impedance loads and 
back-voltages and to be compatible with low-power operational modes. As such, the IDAC utilizes a high output impedance, wide swing design (Fig. 5.13, Fig. 5.14).

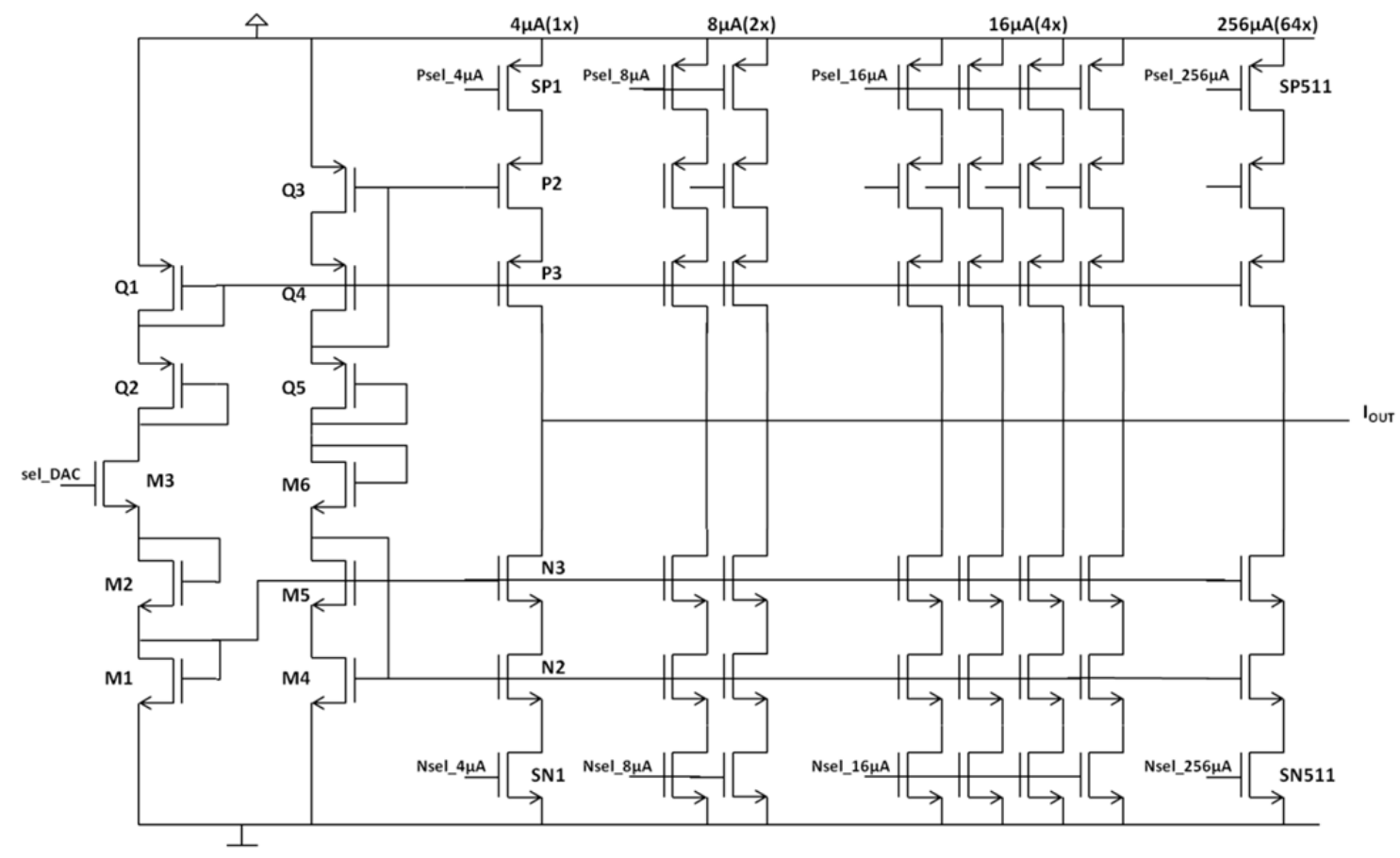

Fig. 5.13: High-swing cascaded I-DAC

\begin{tabular}{|c|c|c|c|c|c|}
\hline \multicolumn{6}{|c|}{ I-DAC Transistor Sizing ( $\mu \mathrm{m}$ ) } \\
\hline \multicolumn{2}{|c|}{ Bias Current } & \multicolumn{2}{|c|}{ Current Sources } & \multicolumn{2}{|c|}{ Switches } \\
\hline $\mathrm{Q} 1 / 2, \mathrm{M} 1 / 2$ & $4.5 / 2.4$ & $\mathrm{P} 2 / 3, \mathrm{~N} 2 / 3$ & $6 / 0.6$ & SP1, SN1 & $12 / 0.6$ \\
\hline Q3/4, M4/5 & $6 / 0.6$ & \multirow{2}{*}{\multicolumn{4}{|c|}{$\begin{array}{l}4 \mu \mathrm{A} \text { transistor string containing } \mathrm{P} 2 / 3, \mathrm{~N} 2 / 3, \mathrm{SP} 1 \text { and SN1 is } \\
\text { duplicated by successive factors of } 2 \text { to generate increasing } \\
\text { multiples of current output. }\end{array}$}} \\
\hline Q5, M6 & $3 / 5.4$ & & & & \\
\hline
\end{tabular}

Fig. 5.14: Transistor sizing (in $\mu \mathrm{m}$ ) of voltage-biased current sources (in $\mu \mathrm{A})$ and DAC switches

The electrode sites are typically have impedance levels as high as $3 \mathrm{k} \Omega$ at $1 \mathrm{kHz}$, but can vary as much as $500 \Omega$ from array to array with changes in the state of the metal deposition tools and activation set-up from run to run. Additionally, biofilms can build up on the sites in-vivo causing fluctuations in the impedance levels. To create a stable IDAC output impedance over the lifetime of array use, a simple cascoding scheme was used in the I-DACs to give their current drivers high output impedance. Although, the 
ASIC will initially be used as a stand-alone tool assess the benefits of high density arrays, eventually it will be incorporated into a fully implantable cochlear prosthetic system. For such a system, it is desirable to operate with the lowest power consumption possible. The ASIC I-DAC is designed with this in mind by using a high-swing bias generator. To incorporate the 8-bit DAC operation into the current driver, MOSFETs SP1-SP511 and SN1-SN511 are controlled using the flag bits generated by the DAC amplitude-select block (sel4 $\mu \mathrm{A}-\operatorname{sel} 256 \mu \mathrm{A})$. The DAC enable flags sel_DAC1-sel_DAC4 are connected to the sel_DAC enable for each corresponding I-DAC to turn them on and off.

Simulations of the I-DAC were done in Cadence Spectre with a power supply of $\pm 2.5 \mathrm{~V}$ and a $3 \mathrm{k} \Omega$ resistance at the output of the driver. For the simulations, the output voltage was varied from $-2.5 \mathrm{~V}$ to $2.5 \mathrm{~V}$ and the response of the output current was measured. Simulated results show a $-3 \mathrm{~dB}$ cutoff of $2.43 \mathrm{~V}$ when outputting $+500 \mu \mathrm{A}$ and less than $-2.45 \mathrm{~V}$ when outputting $-500 \mu \mathrm{A}$ (Fig. 5.15, Fig. 5.16). The output current has a good current stability and a maximum variance of $7.1 \%$ from $+500 \mu \mathrm{A}$ for $-2.5>\mathrm{V}_{\text {out }}<$ $2.21 \mathrm{~V}$. When sinking $-500 \mu \mathrm{A}$, the output has a maximum variance of $6.1 \%$ for $-2.26>$ $\mathrm{V}_{\text {out }}<2.5$.

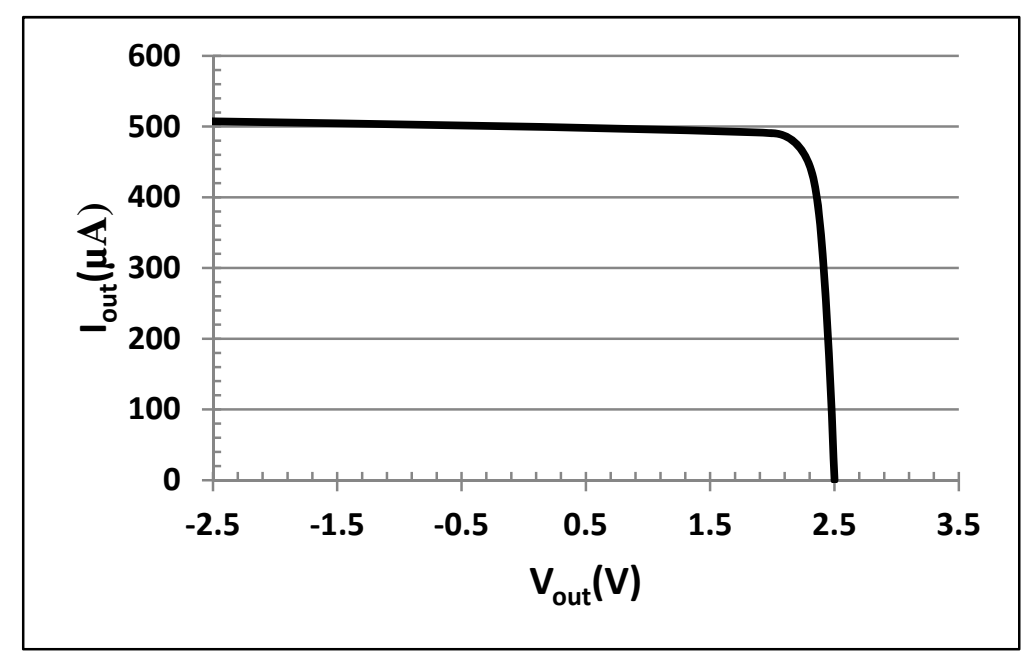

Fig. 5.15: Simulated Iout versus Vout driving $+500 \mu \mathrm{A}$ through a $3 \mathrm{k} \Omega$ resistor with a $\pm 2.5 \mathrm{~V}$ power supply. 


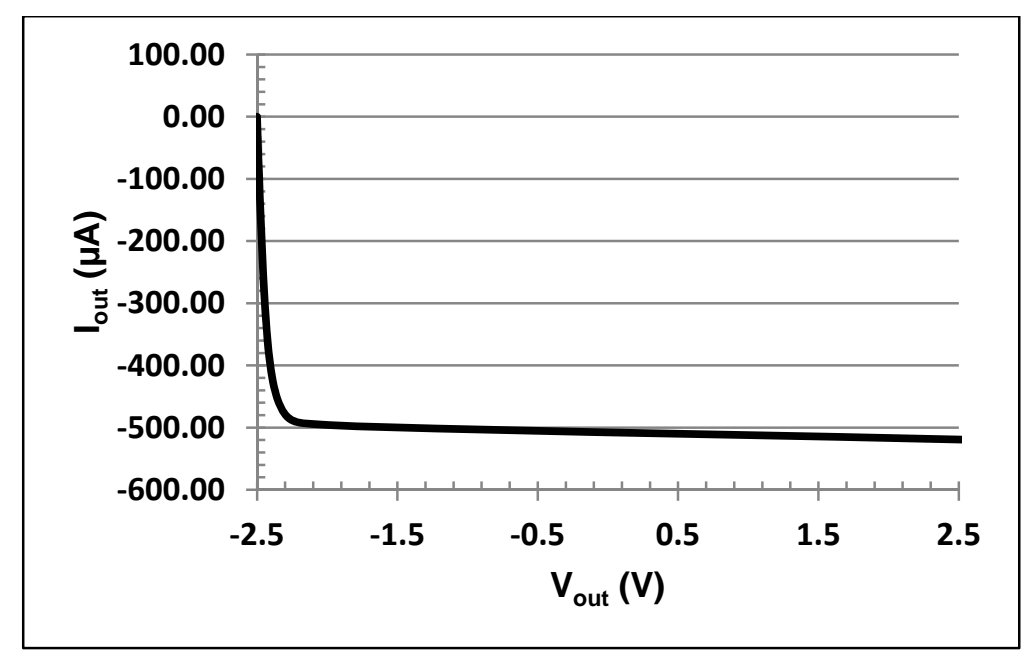

Fig. 5.16: Simulated Iout versus Vout driving $-500 \mu \mathrm{A}$ through a $3 \mathrm{k} \Omega$ resistor with a $\pm 2.5 \mathrm{~V}$ power supply.

The output of the I-DAC was ramped through its full scale range of 0 to $+500 \mu \mathrm{A}$

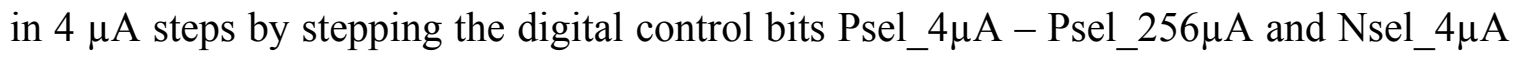
-Nsel_256 $\mu \mathrm{A}$ through their 128 possible 8-bit combinations in time increments of $10 \mu \mathrm{s}$ (Fig. 5.17, Fig. 5.18). A load of $3 \mathrm{k} \Omega$ was attached to the output of the I-DAC. The simulated results show an almost exact match between the ideal output and actual (simulated) current output of the I-DAC as the current ramps from 0 to $+500 \mu \mathrm{A}$ and 0 to $-500 \mu \mathrm{A}$.

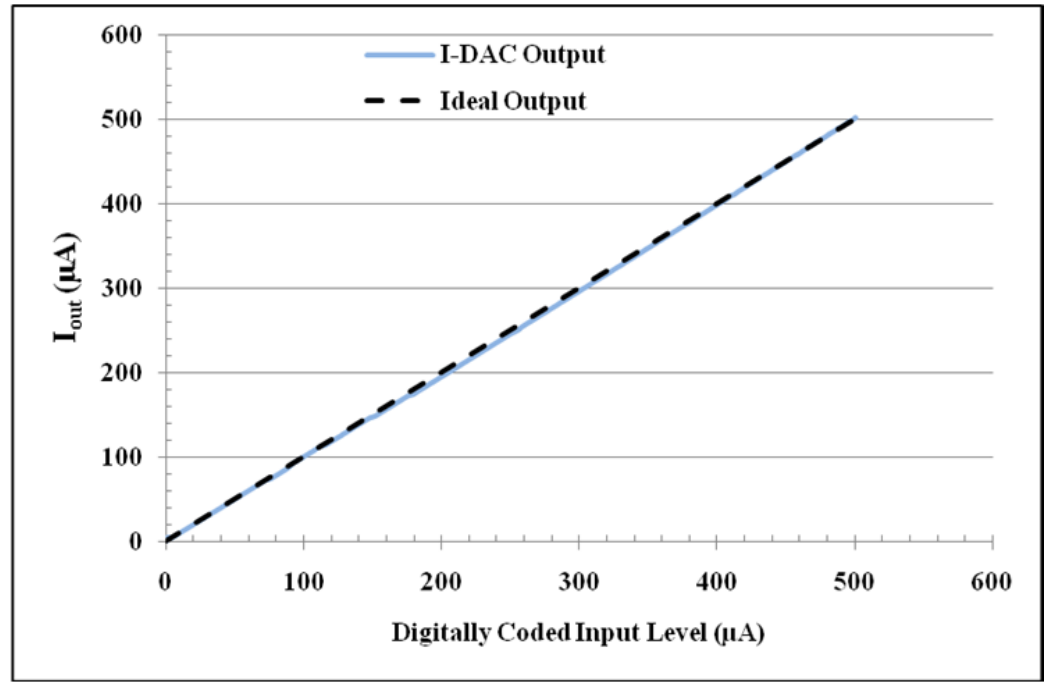

Fig. 5.17: Progression of Iout with digital amplitude selection up to $+500 \mathrm{uA}$ 


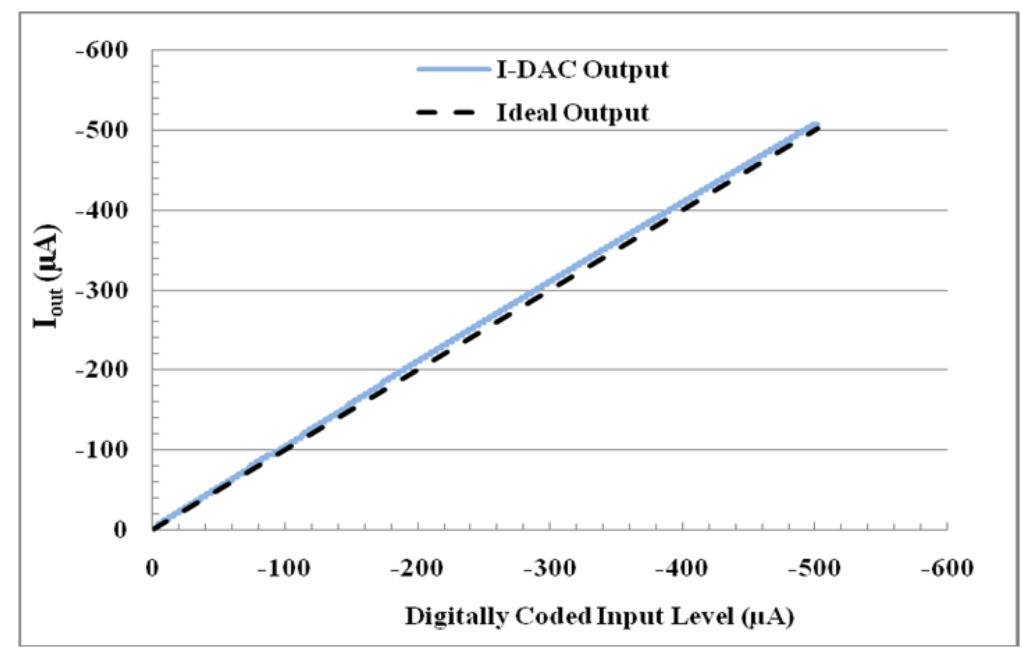

Fig. 5.18: Progression of Iout with digital amplitude selection down to -500uA

The simulated biphasic mismatch, the anodic pulse minus the absolute value of the cathodic pulse, goes from $0 \mu \mathrm{A}$ to $-7 \mu \mathrm{A}$ as the DAC steps through the $\pm 500 \mu \mathrm{A}$ fullscale range. The cathodic pulses draw more current for the same DAC code than their anodic counterparts. However, non-linear effects such as the maximum mismatch was $1.4 \%$ at an anodic output of $+500 \mu \mathrm{A}$ and corresponding cathodic pulse of $-507 \mu \mathrm{A}$ (Fig. 5.19). Although quite small, this small offset could build up quickly at high stimulation rates. To counteract this effect, the ASIC site grounding operation discussed above allows the sites to be grounded, effectively eliminating this buildup of charge.

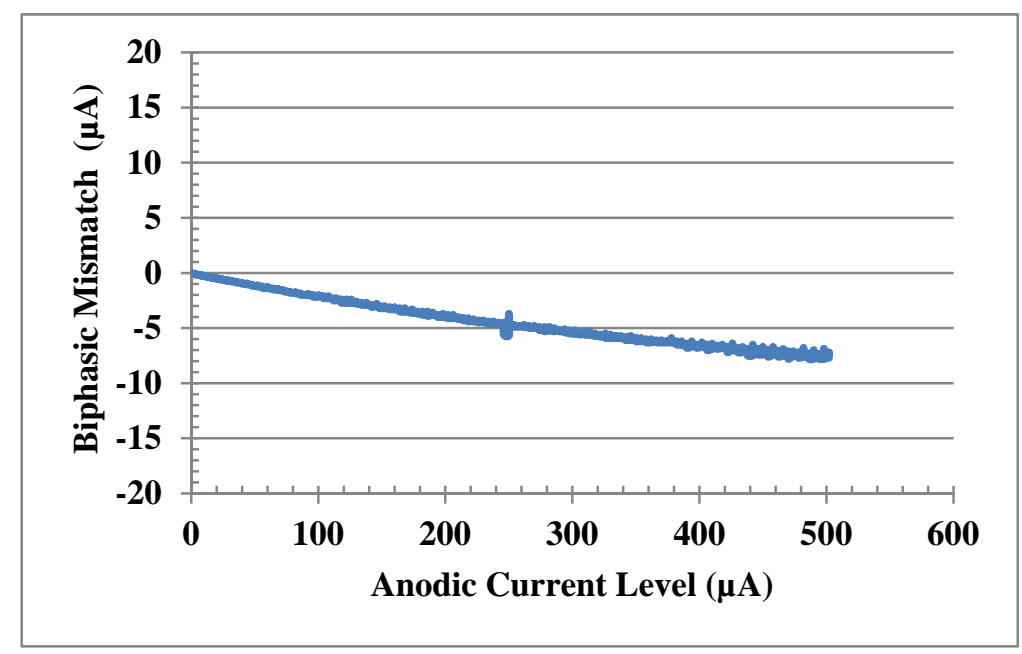

Fig. 5.19: Biphasic offset mismatch over-all output current selection (sourcing) 


\subsubsection{DAC Calibration}

To calibrate any of the four I-DACs, the output can be connected to the DOUT line and a sampling of current levels can be verified ranging from $4 \mu \mathrm{A}$ to $284 \mu \mathrm{A}$. Bits b4, b5, b6 set to 000 result in a DAC calibration command request. The DAC_cal flag bit acts as a selector to connect the input of a 1-to-2 MUX to either the DOUT line (through a pass-gate) or to the input of the switch bank (Fig. 5.20). During a DAC calibration test, the selection lines site1-site 8 are all set to zero and all sites are shut-off. This prevents unwanted nerve activation in calibration mode.

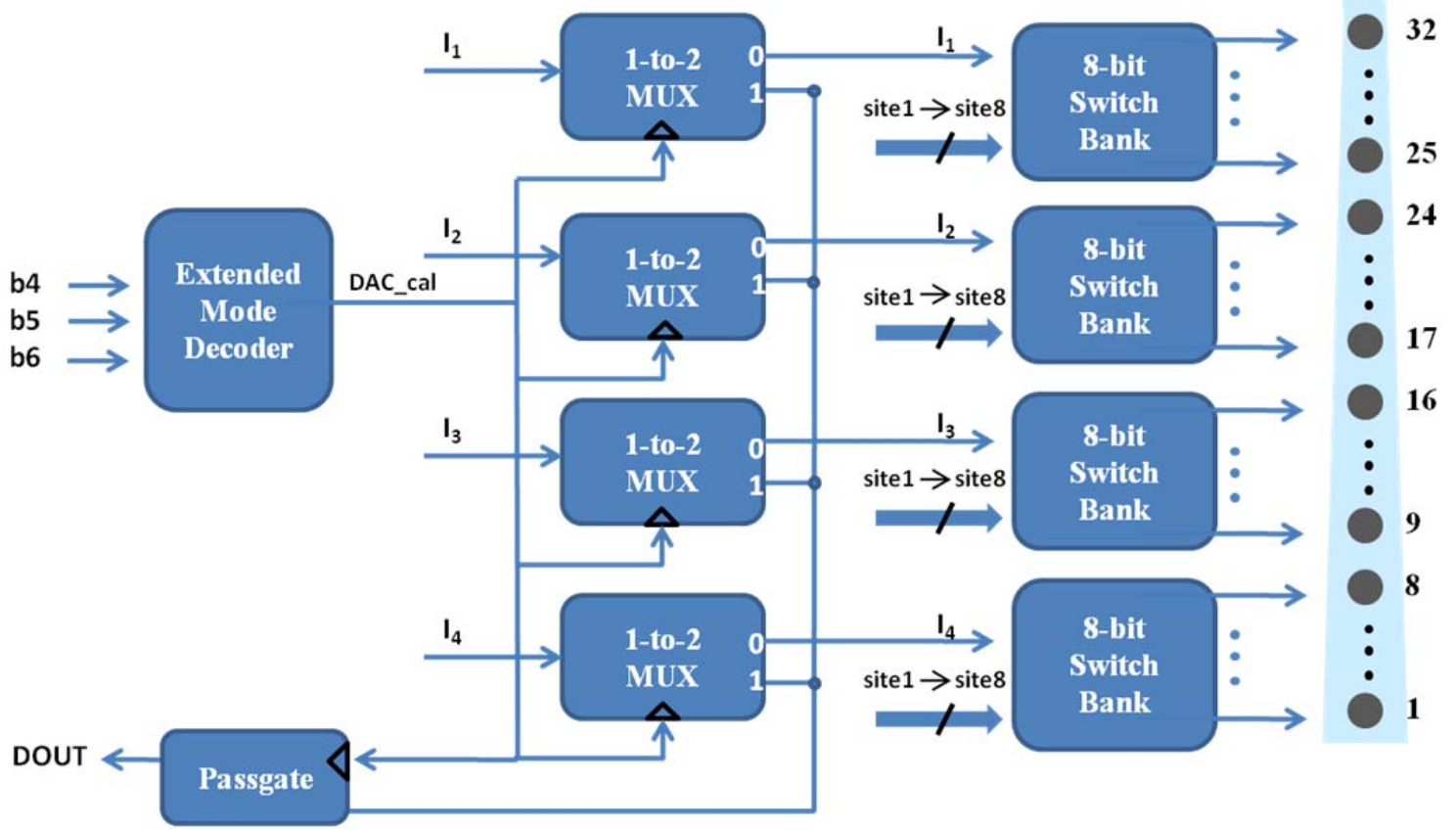

Fig. 5.20: DAC calibration data path

\subsection{Site Selection}

Site selection begins much the same way as DAC selection (Fig. 5.21). Bits b10b11 are decoded into enables for 8-bit latches that hold the flag bits for control of an 8-bit switch bank. The control bits sel_DAC1-sel_DAC4 are the same as the ones used to control the DAC latches. Bits b12-b14 of the command word are passed through a 3-to-8 decoder to generate flag bits site1-site8. These bits are connected to the inputs of the 
eight pass transistors that make up the switch bank for each stimulus channel. Again, level shifting is done before the latches not as a matter of functional necessity but to reduce the number of required level shifters from thirty-six to twelve. The pass transistors of each bank are individually connected to non-adjacent sites across the array in a distributed architecture.

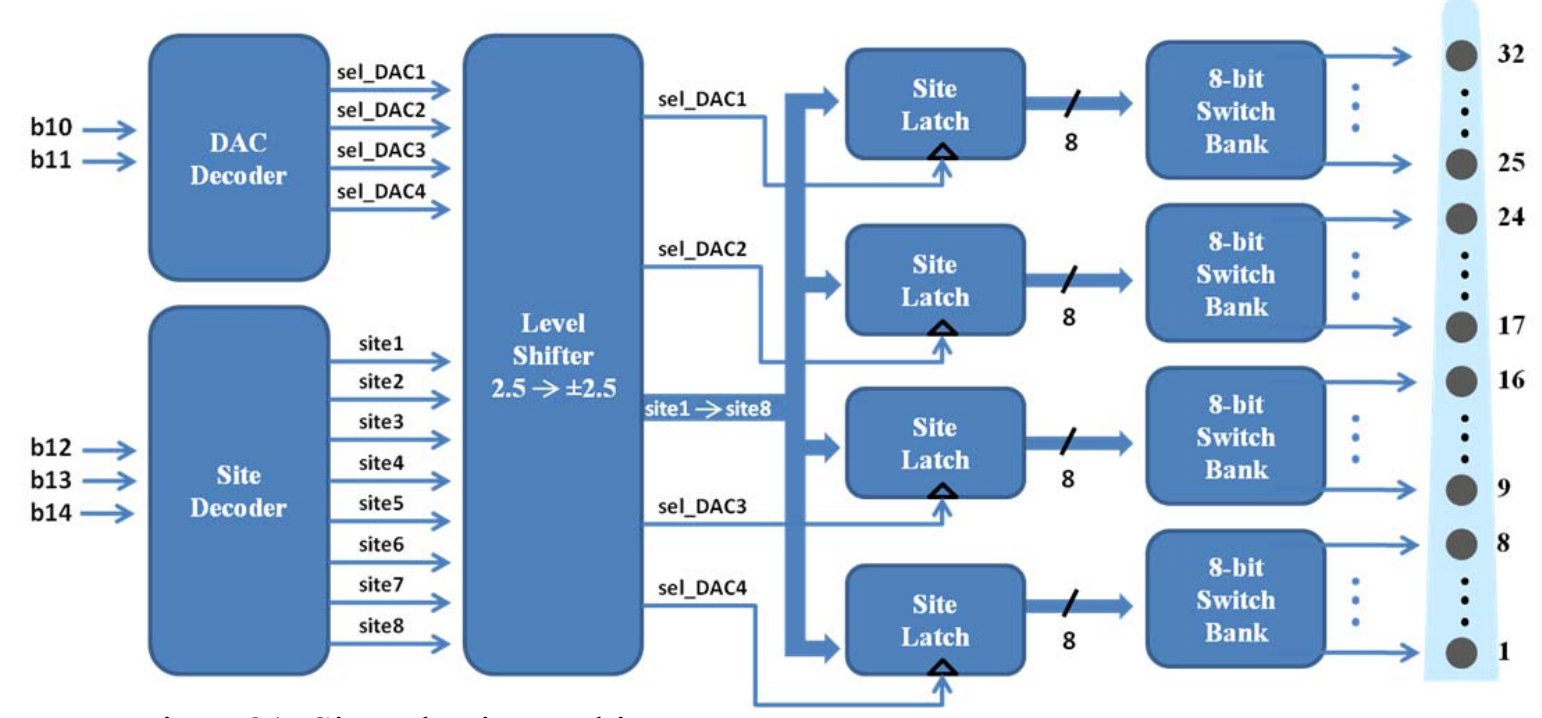

Fig. 5.21: Site selection architecture

\subsection{Site Manipulation and Characterization}

Extended mode operations for testing site impedances, grounding sites and activating sites are built into the ASIC. Bits b4-b5 are decoded to create the selection flags with 010, 011, 100, resulting in site_gnd, site_activ and site_z going high, respectively. The OR of all three flags connects the stimulation site(s) to the DOUT line through a passgate and a 2-to1 MUX if either of them is high. If none of them are high, the sites are connected to currents generated from the I-DACs (Fig. 5.22). 


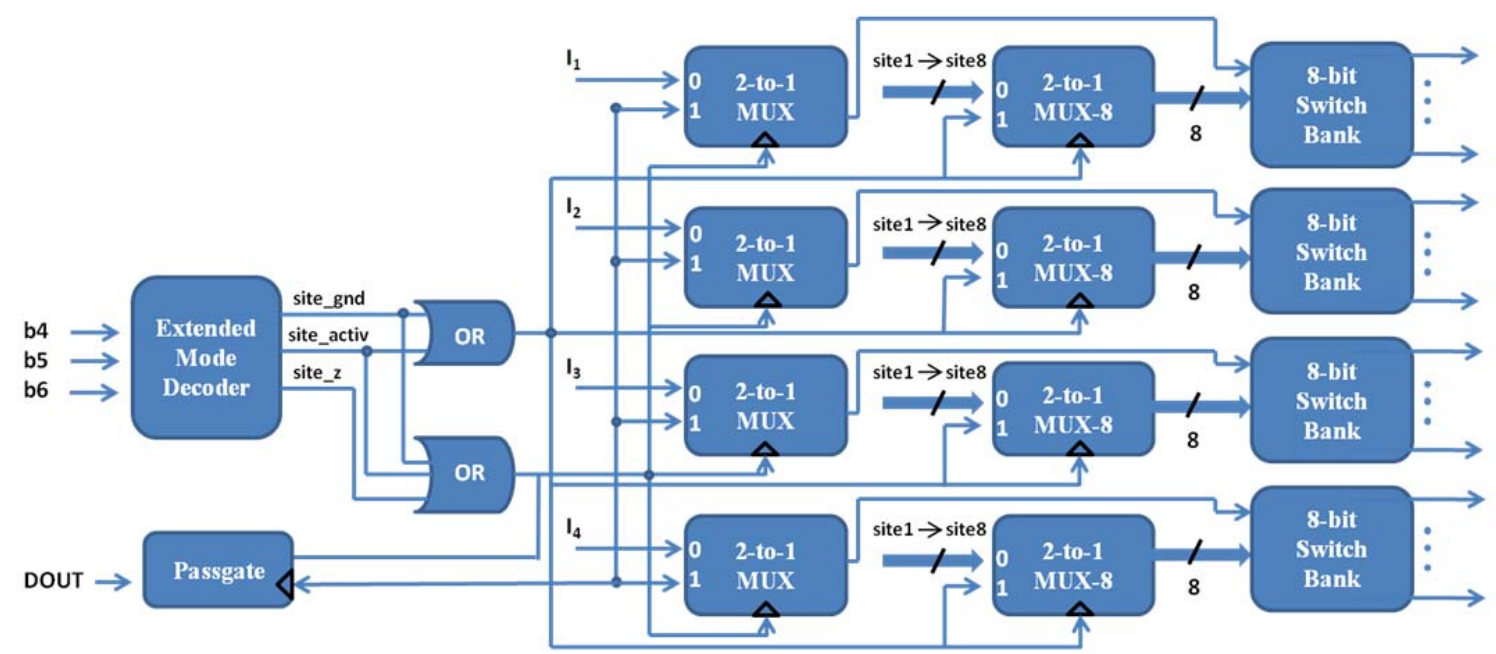

Fig. 5.22: Site impedance ground and activation

Both site impendence and site activation operations are performed simultaneously on all sites across this array. So, when either of these flags are high, all gates of the switch bank pass transistors need to be connected to 1. A 2-to-1 MUX accomplishes this by connecting the switch bank gates to site1-site 8 selection lines or to the OR of site_z and site_gnd. In contrast, a site impedance command measures from individual sites. In the event that a site_z operation is called, both site_gnd and site_activ will be low and the gates of the switch bank will be connected to the site1-site8 selection lines.

\subsection{ASIC Performance}

A 32-site, 4-channel ASIC was fabricated in $0.5 \mu \mathrm{m}$ ON Semiconductor CMOS technology. The chip measured $2.2 \mathrm{~mm}$ X $2.5 \mathrm{~mm}$ with an active circuit area of $1.94 \mathrm{~mm}$ $\mathrm{X} 1.94 \mathrm{~mm}$ (Fig. 5.23). Bench top testing of the chip was done using a LabView interface that generated the DIN, CLK, STB and RST signals. Each signal was generated on the digital output lines of a National Instruments Digital Acquisition (DAQ) PXI 6251 card and level shifted to $\pm 2.5 \mathrm{~V}$ levels with a LT $1057 \mathrm{CN} 8$ op-amps. Timing of the LabView pulse generation was controlled externally using a arbitrary waveform generator. The following sections will detail performance results of the pulse generator and $\mathrm{I} / \mathrm{O}$, and give a summary of the system level performance. 


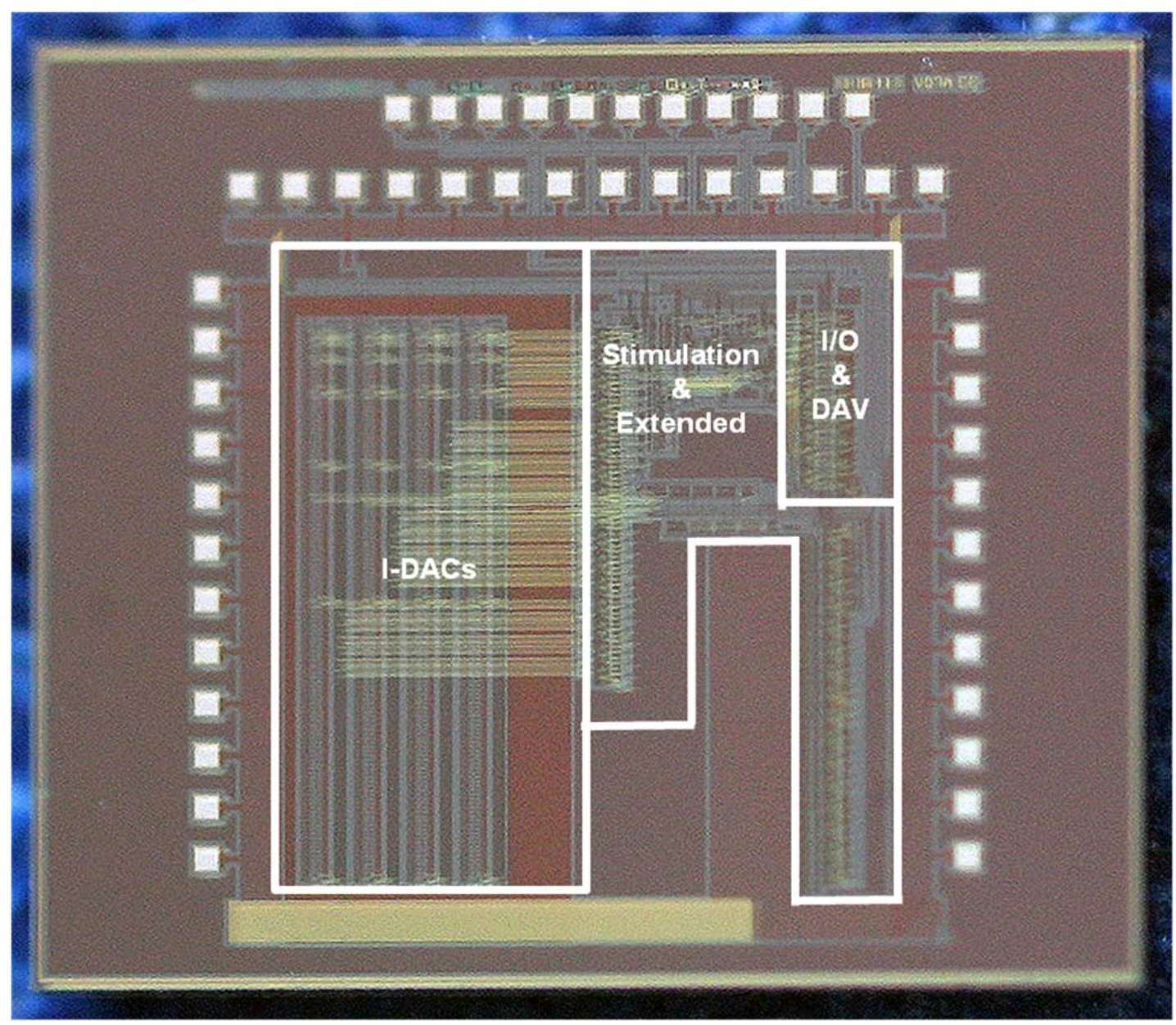

Fig. 5.23: The Cochlear ASIC. Areas not outlined in white are consumed by interconnect.

\subsubsection{ASIC Area}

The size of ASIC is $2.2 \mathrm{~mm} \times 2.5 \mathrm{~mm}$. Roughly $47 \%$ percent of the active circuit area was occupied by the four I-DAC circuits and their interconnects, $25 \%$ by the siteselection and extended mode multiplexers, decoders and shift registers, $4 \%$ by the $\mathrm{I} / \mathrm{O}$ shift register, latch and DAV circuits, and the remaining $24 \%$ by the traces interconnecting the circuit blocks and the ASIC padframe. The padframe of the ASIC was arranged to align with the bond-pads on the backend of the 32-site passive array. In the final assembly, the array backend is ball-bump bonded to thirty two of the thirty eight, $100 \mu \mathrm{m} \times 100 \mu \mathrm{m}$, ASIC pads surrounding the perimeter of the circuit. Of the eleven contact pads along the top of the ASIC, nine pads will be bonded to a parylene cable which extends off the side of the ASIC opposite the array. While the array is 
inserted into the cochlea, the ASIC is designed to fit within the space of the guinea pig otic bulla (which is less than $3 \mathrm{~mm}$ wide), external to the cochlea. In the final realization of a full cochlear system, the nine lead cable will extend outside of the otic bulla and connect to the DSP microcontroller.

Given the space constraints of the guinea pig otic bulla, the previous generation of ASIC was designed to use as small an area as possible. Area conservation was the primary reason for using a shared-DAC architecture; however, in comparison to the shared-DAC architecture of the previous-generation ASIC, the stimulus-generation segment of the ASIC presented here consumed only $16 \%$ more area in dedicating a DAC to each channel. This increase in area can be attributed to the elimination of the sampleand-hold $(\mathrm{S} / \mathrm{H})$ circuitry. In the past, with only one DAC, it was necessary to have both a $\mathrm{S} / \mathrm{H}$ circuit as well as a dedicated current stimulator for each channel, so that up to four different current amplitudes could be driven at any one time. Much of the area consumed by the $\mathrm{S} / \mathrm{H}$ circuitry was devoted to large capacitors which consumed $7,410 \mu \mathrm{m}^{2}$. The total area consumed by the DAC alone was $140,378 \mu \mathrm{m}^{2}$ and each of the four stimulator and $\mathrm{S} / \mathrm{H}$ circuit blocks took up 79, $728 \mu \mathrm{m}^{2}$. In total, the pulse generating circuits consumed approximately $0.46 \mathrm{~mm}^{2}$. Even though the ASIC presented here utilized four separate I-DACs for each channel, the area consumed was only $0.55 \mathrm{~mm}^{2}$.

\subsubsection{I/O}

The I/O block controls the shifting in and latching of command words, system resetting and data verification, as discussed in section 1.3. To test all three of these functions, a command was entered into the ASIC whose parity changed with the shifting in of each bit. To begin the test, the system was reset to zero-out all registers and latches. Next, 16 clock cycles of a $40 \mathrm{kHz}$ clock were used to shift in a 1 on the DIN line. During this operation the STB input was held high (Fig. 5.24). 


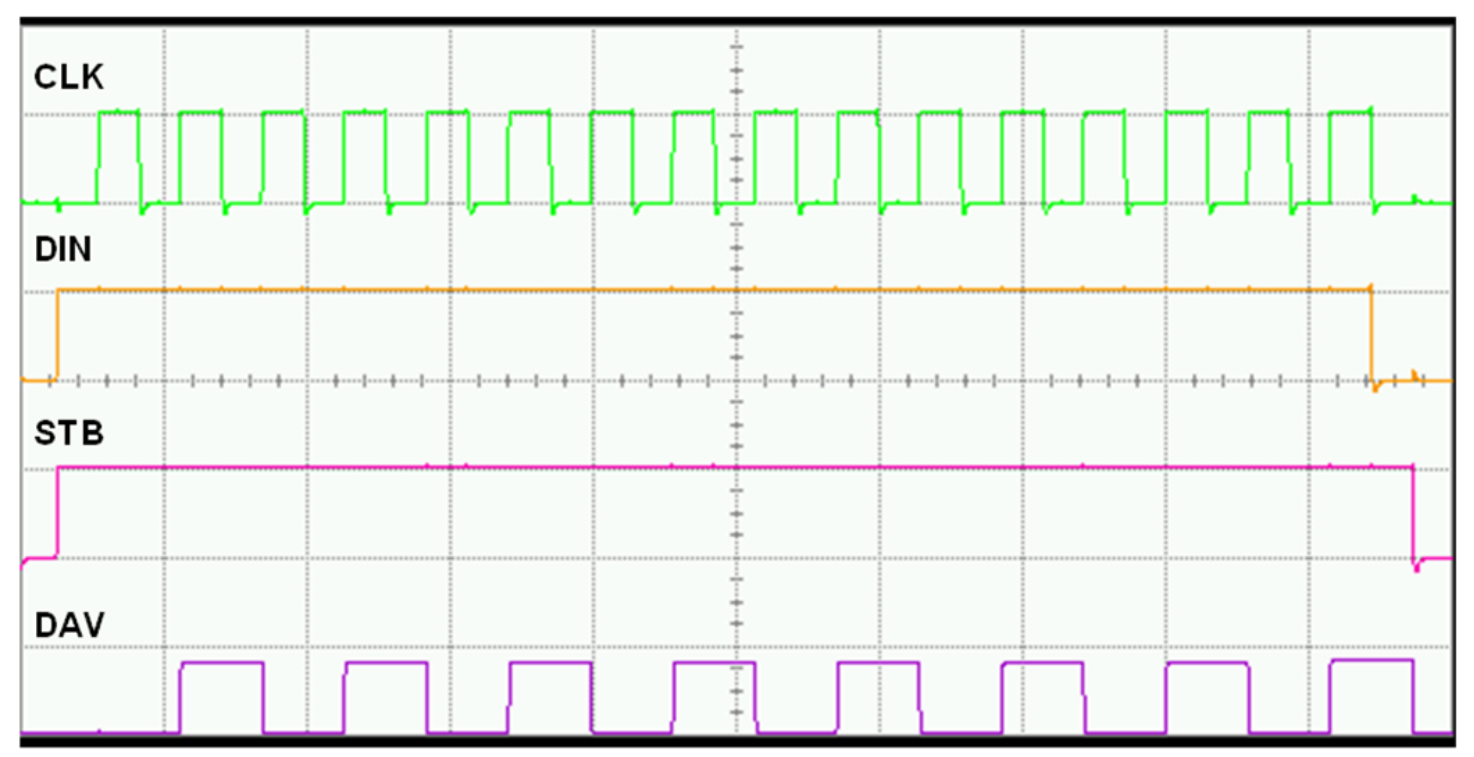

Fig. 5.24: A 40kHz clock shifting in 1 on the DIN line through 16 clock cycles. DAV line alternates from high to low as the parity of the $\mathrm{I} / \mathrm{O}$ latch. The vertical scale is $5 \mathrm{~V} /$ div and the horizontal scale is $87 \mu \mathrm{s} / \mathrm{div}$.

On the first clock cycle a 1 is shifted into b0 of the shift register and passed onto the latch which gets readily updated as STB is high. The output of the $\mathrm{I} / \mathrm{O}$ latch is connected to the input of the data-valid block which runs an even parity check on the 16-bits of the latch. Since the shift register was initialized to all zeros, after the rising edge of the first clock the parity is odd and DAV is low. On the second clock cycle, another 1 is shifted into the I/O register and latch, and now, as the register contains two 1s, DAV switches to 1. For the remaining fourteen clock cycles, DAV alternates between high and low as each additional 1 changes the parity from even to odd. After the rising edge of the $16^{\text {th }}$ clock cycle, all bits of the register and latch are 1 and DAV remains high until STB goes low.

Both the shift-register and the DAV command work as expected. Commands were successfully shifted into the I/O at speeds up to $500 \mathrm{kHz}$, limited only by the test setup. Likewise, DAV was able to correctly asses the parity of the command words and signal the validity on its output line. Additionally, DAV responded correctly to the rising and falling of the STB signal by only switching high when the STB signal was also high. Due to a misrouting of STB in the layout, it was necessary for STB to be held high during shifting in of command words. This altered the hand-shaking protocol for the ASIC and 
command input sequence, but still allowed for the successful shifting and latching of the 16-bit command words.

\subsubsection{Stimulation Performance}

To test power supply variation a $3 \mathrm{k} \Omega$ resistor representing an electrode was chosen because it was on the high-end of the measured $1 \mathrm{kHz}$ site-to-contact impedances of the IrO electrodes. The I-DAC's response to shifts of $\pm 10 \%$ in the power supply were measured (Fig. 5.25). Such shifts can cause the current output to increase or decrease. Both VDD and VSS where kept equal but opposite, and varied in steps of $0.05 \mathrm{~V}$ from $\pm 2.25 \mathrm{~V}$ to $\pm 2.75 \mathrm{~V}$. For a $-200 \mu \mathrm{A}$ output, increasing the supply rails to $\pm 2.75 \mathrm{~V}$ caused a $1 \%$ increase in the current output to $-202 \mu \mathrm{A}$. Decreasing the power supply by $10 \%$ to $\pm 2.25 \mathrm{~V}$ had a greater effect on the current output and decreased it by $5 \%$ to -190 $\mu \mathrm{A}$. As the supply decreases it reduces the compliance voltage, and $\mathrm{V}_{\text {out }}$ has a greater effect on the current output, resulting in a bigger decline in $\mathrm{I}_{\text {out }}$.

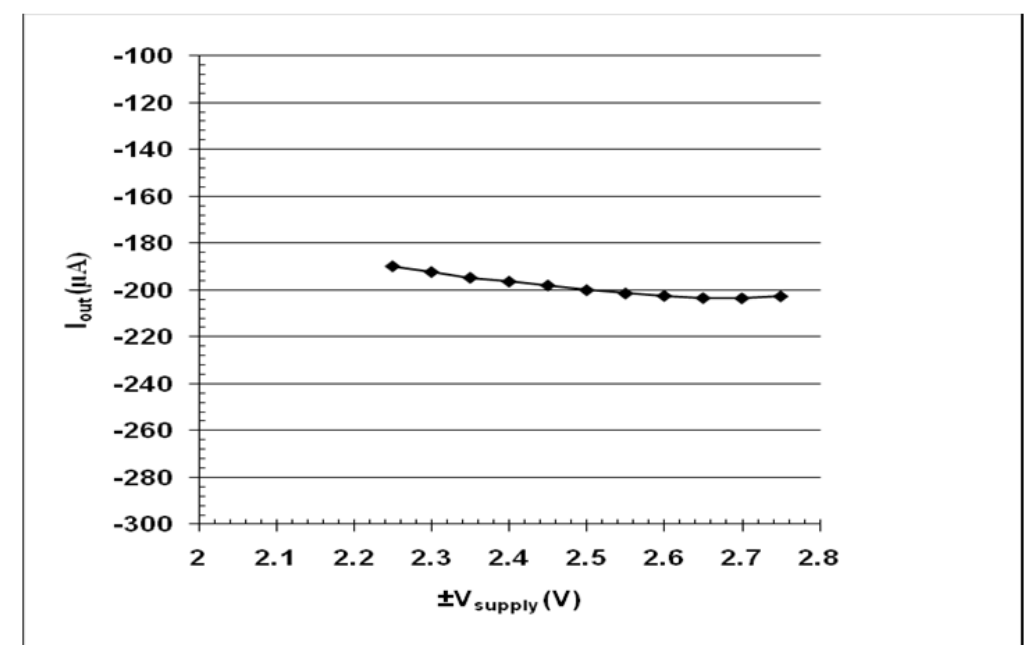

Fig. 5.25: Output current versus supply voltage for a change in the supply voltage of $\pm 10 \%$. The amplitudes of VDD and VSS were equal but opposite.

The I-DAC could be adjusted to current values from $-500 \mu \mathrm{A}$ to $+500 \mu \mathrm{A}$; however, the ramping of current was not always linear as a function of increasing code and a decrease instead of increase in current level was seen for some digital codes. For subsets of current values within the $\pm 500 \mu \mathrm{A}$ range the ramping was linear in 
progression. Although the two functional blocks could not be isolated from each other, it seemed likely that the linearity shortcomings had more to do with the current amplitude decoding block than the I-DAC itself. The command word bits did not correlate to the correct amplitude levels in all cases. However, by modifying the command word protocol nearly all levels could be attained.

Using a $500 \mathrm{kHz}$ clock, biphasic pulses were delivered to site locations. The command bits were clocked into the ASIC, and the resulting biphasic pulses were measured at the site locations across a $3 \mathrm{k} \Omega$ resistor. The biphasic pulses had a mismatch of $16 \%$ at $500 \mu \mathrm{A}$, with the absolute value of the cathodic pulse being higher than the anodic pulse. This mismatch decreased for smaller current outputs and is likely due to the mismatches between the drain currents of the NMOS and PMOS halves of the IDAC. As the current output increases, these mismatches compound as more $4 \mu \mathrm{A}$ transistor-strands are driven. To counteract this charge imbalance, the cathodic pulse could be shortened in duration relative to the anodic to deliver less charge.

The back voltage response to ASIC driven current through an IrO electrode array site in PBS was measured (Fig. 5.26, Fig. 5.27). The response of IrO electrodes to a current pulse are as expected with an initial voltage jump/drop from the resisistive current flow through the interconnect lines and external cabling, followed by a capacitive charging of the double layer. The $3 \mathrm{~dB}$ cut-off voltage for the output voltage was measured to be greater than $2.35 \mathrm{~V}$ which is close to the simulated cut-off of $2.43 \mathrm{~V}$. As such, the ASIC I-DAC is compatible with the back voltages of $180 \mu$ m-diameter IrO sites. Back voltages recorded for those sites in-vivo are less than $2 \mathrm{~V}$ for stimulus pulses up to $500 \mu \mathrm{A}$ with pulse durations of less than $25 \mu \mathrm{s}$. 


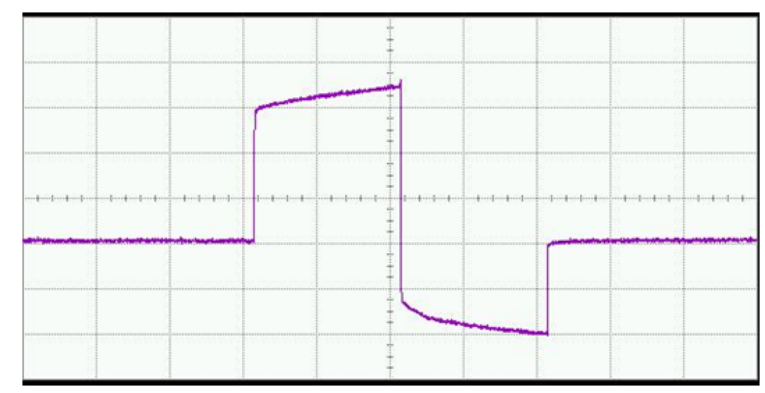

Fig. 5.26: Back voltage response to a $200 \mu \mathrm{s}$ and $300 \mu \mathrm{A}$ pulse. Backvoltage is roughly $0.3 \mathrm{~V}$ off a $0.5 \mathrm{k} \Omega \mathrm{IrO}$ site. The vertical scale is $100 \mathrm{~m} \mathrm{~V} / \mathrm{div}$ and the horizontal scale is $100 \mu \mathrm{s} / \mathrm{div}$.

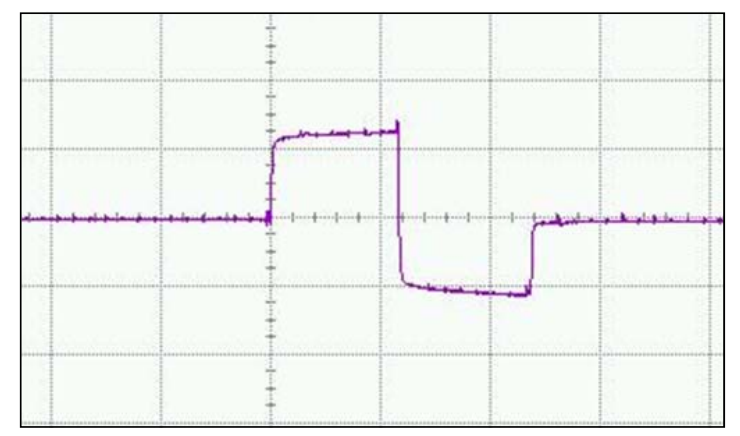

Fig. 5.27: Back voltage response to a $25 \mu \mathrm{s}$ and $128 \mu \mathrm{A}$ pulse. Backvoltage is roughly $0.13 \mathrm{~V}$ off a $0.5 \mathrm{k} \Omega \mathrm{IrO}$ site. The vertical scale is $100 \mathrm{mV} /$ div and the horizontal scale is $20 \mu \mathrm{s} / \mathrm{div}$.

\subsubsection{ASIC Performance Summary}

In final use, the ASIC will be bonded to the stimulating array through a cable and surgically positioned in the otic bulla, a boney cavity outside the cochlea. The space of the otic bulla roughly $2.5 \mathrm{~mm}$ by $8 \mathrm{~mm}$. A 32-site, 4-channel ASIC was realized in $0.5 \mu \mathrm{m}$ ON Semiconductor technology. The chip measured $2.2 \mathrm{~mm}$ X $2.5 \mathrm{~mm}$, operated from a $\pm 2.5 \mathrm{~V}$ supply, and generated biphasic pulses of greater than $\pm 500 \mu \mathrm{A}$. The maximum power consumption of the ASIC was $2.5 \mathrm{~mW}$ when outputting $500 \mu \mathrm{A}$ and operated with back voltages exceeding $\pm 2 \mathrm{~V}$. The measured maximum biphasic mismatch of the pulses generated was $16 \%$. The ASIC was functional at CLK speeds up to $500 \mathrm{kHz}$. Greater speeds could be realized with the ASIC, and in simulations it operated at CLK speeds 
above 10MHz. However, for most stimulation protocols the timing parameters would not require speeds above $500 \mathrm{kHz}$. The timing parameters that contribute to frequency selectivity and overall sound appreciation are the pulse width for each phase (anodic, cathodic), the interphase delay, and the interpulse delay, which is the delay between successive biphasic pulses. In practice, the minimum pulse widths used are around $25 \mu \mathrm{s}$ and studies show pulse durations above $50 \mu$ s are usually preferred by patients. Although the interphase delay is typically zero for biphasic pulses, it depends on the rate of stimulation and the given pulse width. Stimulation rates are considered high around the 5000 pulses per second (pps) range and usually the rate of stimulation is well below 2500 pps [11]. Based on the measured results for the ASIC, it can generate biphasic pulse widths as low as $16 \mu$ s with zero interphase delay at rates as high as 32000 pps.

Table 5.1: Summary of ASIC Performance

\begin{tabular}{|c|c|}
\hline \multicolumn{2}{|c|}{ Cochlear ASIC Performance Summary } \\
\hline Supply Voltage & $\pm 2.5 \mathrm{~V}$ \\
\hline Power Consumption & $\begin{array}{l}2.5 \mathrm{~mW} \text { (at } 500 \mu \mathrm{A} \text { output) } \\
0.17 \mathrm{~mW} \text { (extend mode) }\end{array}$ \\
\hline Max Stimulation Rate & 32000 pps \\
\hline Compliance Voltage & $2.35 \mathrm{~V}$ \\
\hline Biphasic Mismatch & $16 \%$ \\
\hline $\begin{array}{l}\text { Biphasic Stimulation } \\
\text { Level }\end{array}$ & $\begin{array}{l} \pm 500 \mu \mathrm{A}(8 \text {-bit resolution, } \\
4 \mu \mathrm{A} \text { steps) }\end{array}$ \\
\hline Minimum Pulse Width & $16 \mu s$ \\
\hline Area & $2.2 \mathrm{~mm} \times 2.5 \mathrm{~mm}$ \\
\hline
\end{tabular}

\subsection{References}

[1] Marsman, E.D.; Senger, R.M.; Carichner, G.A.; Kubba, S.; McCorquodale, M.S.; Brown, R.B.; , "DSP architecture for cochlear implants," IEEE International Symposium on Circuits and Systems , pp.657-660, 2006. 
[2] Bhatti, P., "A high-density thin-film electrode array for a cochlear prosthesis," in Electrical Engineering and Computer Science, Ph.D. Ann Arbor: The University of Michigan, 2006.

[3] Bhatti, P. T.; Wise, K.D., "A 32-site 4-channel high-density electrode array for a cochlear prosthesis," IEEE journal of solid-state circuits, vol. 41, pp. 2965 - 2973, 2006.

[4] Snyder, R. L.; et al., "Cochlear implant electrode configuration effects on activation threshold and tonotopic selectivity," Hearing Research, vol. 235, pp. 23 $-38,2008$.

[5] Gingerich, M., "Multi-dimensional microelectrode arrays with on-chip CMOS circuitry for neural stimulation and recording," in Electrical Engineering and Computer Science, Ph.D. Ann Arbor: The University of Michigan, 2002.

[6] Boston Scientific, "HiRes with fidelity 120 sound processing", A Report from Advanced Bionics, The Auditory Business of Boston Scientific, 2006.

[7] A. B. Corporation, "Increasing spectral channels through current steering in HiResolution bionics ear users," Aug. 2011, http://www.advancedbionics.com

[8] HONET, C., "Pitch Steering with Sequential Stimulation of Intracochlear Electrodes." Cochlear research report, 2006.

[9] Jill B.; Holden, Laura K.; Reeder, Ruth M.; Skinner, Margaret W., "Cochlear Implants, Speech Recognition in Cochlear Implant Recipients: Comparison of Standard HiRes and HiRes 120 Sound Processing", Otology \& Neurotology, vol. 30, pp. 146-152, 2009 


\section{Chapter 6}

\section{SUMMARY, CONTRIBUTIONS, AND FUTURE WORK}

Cochlear implants for the deaf are the most successful neural prostheses; however, pitch perception remains relatively poor. The size of the scala tympani into which the stimulating electrodes are inserted limits their number to about twenty using conventional wire-bundle arrays. These arrays are also hand assembled and relatively expensive. Thin-film arrays can offer significant advantages by increasing the number of sites (increased pitch specificity), reducing damage to residual hearing with smaller and more flexible arrays, and allowing deeper insertion (greater pitch range). However, any thin-film array must be robust enough for safe insertion into the helical cochlea, stiff enough for deep insertion, and have a modiolus-hugging curl to position the sites close to receptor cells and reduce insertion trauma. Various thin-film cochlear arrays have been

developed which address a few of these requirements; however, none so far have addressed them all. The work presented in this thesis has made significant contributions to addressing each of these requirements, building on the success of a previousgeneration Michigan array by increasing the reliability, usability and functionality of the prosthesis.

A robust and flexible 32-site prototype cochlear electrode array for a 128-site human array has been developed. Molded and thin-film backings for this array have been created for positioning the array inside the cochlea and close to the modiolus wall. The array has been integrated into the commercial molding process of Cochlear, Ltd., as well as into a custom molding process created as part of this thesis. The ultimate goal of array development is the realization of fully batch-fabricated devices that are compatible with commercial injection molding processes, allowing fast adoption to existing markets.

As an intermediate step between molded and monolithic backings, hybrid designs have been implemented. Both PET and PLA stiffeners have been designed with collaborators at MTU and partially integrated into the parylene arrays. Cochlear arrays 
made from more brittle materials had difficulties being combined with various hybrid backings and would tend to break. However, the flexibility of the parylene arrays and the ability to tailor this flexibility makes the devices compatible with a broader range of backing structures. Arrays combined with flattened PET tubes were used with AOS insertion methods to achieve full insertion of cat arrays. Although these hybrid designs show promise in a variety of areas, it would be even better if such designs could be monolithically integrated into the parylene electrode arrays. Monolithic integration gives greater control over mechanical characteristics of the array and cuts back on the amount yield-reducing post-processing steps.

Monolithic backings that include flexible parylene rings and self-curling parylene-metal-parylene layers have been created. Two types of curling structures were used: one that relied primarily on residually-stressed metal layers (Ti-Ir/Ti-Pt), and another that relied on residually-stressed parylene layers. The metal-based devices performed the best in simulations, but were difficult to fabricate since the high stress TiIr layer was prone to delaminating and cracking. Alternatively, the parylene-based curling layers were implemented by offsetting the thickness of the substrate and that of the top layer of parylene surrounding the array interconnects. With this strategy, the fabricated arrays achieved a minimum radius of curvature of $2 \mathrm{~mm}$. The curled arrays required the addition of a channel on the back of the parylene arrays so that they could be temporally straightened for insertion into the cochlea. Such channels were created using discrete parylene rings that did not impede the built-in curvature of the array. The rings could be produced with a limited increase in stiffness (less than 15\%) over that of a flat array. Although, pre-curved arrays offer superior modiolus-hugging features, many surgeons prefer straight arrays and the majority of companies produce them. Straight parylene cochlear arrays were produced using a slotted parylene tube on the back of the arrays. Simulations show that such a tube could be graded in stiffness from $0.2 \mathrm{k} \cdot \mathrm{N} / \mathrm{m}^{2}$ to $1.4 \mathrm{k} \cdot \mathrm{N} / \mathrm{m}^{2}$ with a seven-fold increase in rigidity over a flat parylene array.

Both flat and curved parylene arrays were implanted in-vivo in cat and guinea pig animal models. Full insertions of all 32 -sites were achieved in both animals and with the deepest insertions to date (more than $8 \mathrm{~mm}$ ) in some cases. Guinea pig arrays of graded stiffness have achieved $6 \mathrm{~mm}$ insertion depths. These soft and flexible arrays achieved 
atraumatic implantations with no visible damage to the scala media. With handassembled commercial arrays, because of their high stiffness, it is not uncommon for them to cross over the scala media during insertion, and this not only damages residual hearing but also decreases device performance. The work presented here towards highly flexible and soft arrays has the potential to significantly reduce the surgical trauma. With less trauma and damage to residual hearing, many more patients could benefit from cochlear implants that presently cannot as a result of their residual hearing. This is especially true of older implant users who have lost hearing as a result of age but cannot have their hearing improved with the use of cochlear implant because of the threat that the stiff hand-assembled arrays present to their residual hearing. Companies such as MED-EL are actively working towards addressing this issue [1].

A hybrid ASIC will be integrated into the passive parylene arrays. The cochlear ASIC will increase the functionality of the high-density cochlear electrode array in three ways: (1) It will reduce the voltage drops from the receiver of the prosthesis to the electrode array by decreasing the connecting cable from 128 tightly-pitched lead lines to just 9 lines, which can be made much wider. (2) It will reduce the stiffness and increase the robustness of the connecting cable by reducing the number of leads going down it. (3) It will create an active array capable of handling a diverse range of site configurations, pulse train specifications, and current stimulating schemes.

The ASIC is primarily intended to be used as a research tool to assess the limits of pitch discrimination with high-density electrode arrays. It contains a four-channel pulse generator and has built-in functions for assessing the state of the high-density system. Some of these functions are diagnostics for verifying correct operation of the ASIC I/O and pulse generation circuitry, while others are geared toward monitoring and altering the state of the electrode array by measuring site impedances, grounding sites and activating sites post hybrid assembly. Operation is controlled by a 16-bit command word, a handshaking routine is employed for timed chip-to-chip communication, and all operations are generated on-chip without using off-the-shelf components or external pulse generators. A 32-site, 4-channel ASIC was realized in $0.5 \mu \mathrm{m}$ technology. The ASIC allows for a wide range of multipolar configurations using a distributed DAC and channel architecture. It fits within the space of the otic bulla having a size of $2.2 \mathrm{~mm} \mathrm{X}$ 
$2.5 \mathrm{~mm}$, operates froma $\pm 2.5 \mathrm{~V}$ supplies at CLK speeds up to $500 \mathrm{kHz}$, and generates biphasic pulses of greater than $\pm 500 \mu \mathrm{A}$. The maximum power consumption of the ASIC is $2.5 \mathrm{~mW}$ when outputting $500 \mu \mathrm{A}$, remaining functional with back voltages exceeding $\pm 2 \mathrm{~V}$. The bond pad area of the ASIC is designed to be match the backend of the parylene arrays. The passive arrays extend off of one end of the ASIC and a nine-lead cable extends off of the other.

\subsection{Contributions}

This work has contributed significantly to the development of cochlear prostheses and the fundamental research of neuroscientists investigating the performance of such devices. The principal contributions of this work were:

- Development of a flexible and robust thin-film cochlear electrode array with highdensity sites sitting on $250 \mu \mathrm{m}$ centers.

- Mechanical flex testing of parylene electrode arrays to prove their suitability for cochlear applications as well as others that require a high degree of twisting (spinal cord stimulators, nerve cuff electrodes, etc.)

- Integration of a thin-film array with a commercial molding process capable of providing the fast path to clinical use as part of the worldwide cochlear implant market.

- Integration of a thin-film array with a custom molding process for animal-specific studies.

- Development of monolithically-formed curl structures. Such structures give the arrays modiolus-hugging curl and eliminate the need to curl the arrays postfabrication with messy low-yield methods. 
- Development of an array-on-channel process. For the cochlear arrays, these channels can be used to in conjunction with stylet wires (active or otherwise) to position the arrays inside the cochlea. They also provide stiffness to the arrays that can be graded along their length. The need for stiffeners for polymer microelectrode arrays extends beyond the cochlear application and is quite necessary for neural probes as well. The channels can also be used for pneumatic actuation, drug delivery, pressure monitoring and more.

- First-ever full insertion of a thin-film array in-vivo. The highest insertion depths to date (greater than $8 \mathrm{~mm}$ ) were achieved. This will provide scientists studying the effects of cochlear implants greater access to the low-frequency regions of hearing.

- Development of a cochlear array ASIC that is capable of stimulation using $\mathrm{BP}+\mathrm{N}$ and $\mathrm{TP}+\mathrm{N}$ configurations for most values of $\mathrm{N}$ less than thirty-two. This ASIC will allow for a thorough assessment of what a high-density array can do to increase frequency selectivity.

\subsection{Future Work}

Many significant advances have been made towards a thin-film cochlear prosthetic array and stimulation ASIC; however, much more work needs to be done towards a fully-functional and well-characterized prosthetic system. The following action items are deemed most important:

- Accelerated soak tests should be done to fully characterize the performance of the heat-treated backed parylene arrays. Although others have shown the electrical reliability of this material over time, no one has investigated the mechanical performance of parylene-metal-parylene stacks using accelerated soak tests. 
- In-vivo implants of the 32-site arrays need to be carried out to assess the multipolar performance of the high-density arrays.

- Chronic in-vivo implants of the 32-site arrays need to be done to determine performance in cat and guinea pig animal models.

- Acute studies of pre-curved cat electrode arrays could be conducted to assess how well the arrays hug the modiolus in-vivo. Due to the size constraints of the guinea pig animal model, the AOS technique could not be utilized; however, the cat cochlea more readily resembles the human in size, and AOS could be done with the ringed-backed arrays as it was done with the PET-backed arrays. As part of this assessment, the frequency selectivity and power consumption of the implants should be studied in the cat model with a curved versus a straight array.

- Multilayer interconnect schemes using parylene or oxide as a dielectric layer should be investigated as part of the fabrication of a 128-site human array. Due to area constraints, multilevel metal will be necessary for the full-scale human array.

- The ASIC should be redesigned to fix known errors with the I/O, DAC amplitudeselection and channel-selection blocks. The I-DAC did not perform as well as expected in terms of output impedance, performing more like a non-cascoded IDAC. This issue needs to be investigated further to assess the cause and redesign for a second run.

- A packaging scheme for hermetically sealing the array-bonded ASIC, involving metal and parylene encapsulation, should be implemented and tested via saline soak tests and in-vivo implants.

- A National Instruments Labview GUI for setting desired site configurations, pulse widths and pulse rate should be developed as a tool for the further study of active high-density arrays. With this GUI, the integrated ASIC and array should be tested in-vivo in both chronic and acute implants. 